\title{
Stereoselective Synthesis of Cis- and Trans- Tetrasubstituted Vinyl Silanes Using a Silyl-Heck Strategy and Hiyama Conditions for Their Cross- Coupling
}

\author{
Michael F. Wisthoff, Sarah B. Pawley, Andrew P. Cinderella, and Donald A. Watson* \\ Department of Chemistry and Biochemistry, University of Delaware, \\ Newark, Delaware 19716, United States
}

\section{Supporting Information}

Index

Page

1. General Experimental Details

2. Instrumentation and Chromatography

3. Synthesis of Alkynes

S3

4. Synthesis of Alkylzinc Halides

5. Synthesis of Tetrasubstituted Vinylsilanes via Carbosilylation Reactions

6. Synthesis of Tetrasubstituted Alkenes via Hiyama Cross-Coupling

S21

7. Additional Optimization Details

S22

8. Examination of Silicon Substitution

S24

9. References

$\mathrm{S} 24$

10. Spectral Data

S25 


\section{General Experimental Details:}

1,4-Dioxane, tetrahydrofuran (THF), and triethylamine $\left(\mathrm{Et}_{3} \mathrm{~N}\right)$ were dried on alumina according to published procedures ${ }^{1}$. Trimethylsilyl iodide was purchased from Gelest, distilled into a Straus flask containing copper beads, and stored under nitrogen. Dimethylbenzylsilyl iodide, ${ }^{2}$ dimethylphenylsilyl iodide, ${ }^{2}$ and diphenylmethylsilyl iodide ${ }^{3}$ were prepared according to published procedures, distilled into a Straus flask containing copper beads, and stored under nitrogen. Bis(3,5-di-tertbutylphenyl)(tertbutyl)phosphine (JessePhos), ${ }_{4}$ (JessePhos) ${ }_{2} \mathrm{PdCl}_{2},{ }^{5}$ tris(3,5-di-tertbutylphenyl)phosphine (DrewPhos), ${ }^{3}$ (DrewPhos) ${ }_{2} \mathrm{Pdl}_{2},{ }^{3} \quad(\mathrm{COD}) \mathrm{Pd}\left(\mathrm{CH}_{2} \mathrm{TMS}\right)_{2},{ }^{6} \quad\left[\left(\mathrm{C}_{6} \mathrm{~F}_{5}\right)_{3} \mathrm{P}\right]_{2} \mathrm{PdCl}_{2},{ }^{7} \quad$ (E)-6-iodo-2-hexene, ${ }^{8}$ 2-(3-iodopropyl)4,4,5,5-tetramethyl-1,3,2-dioxaborolane, ${ }^{9}$ iodomethylbenzene, ${ }^{10}$ bis(4-methoxyphenyl)acetylene, ${ }^{11}$ bis(3methylphenyl)acetylene, ${ }^{12} \quad$ bis(4-fluorophenyl)acetylene, ${ }^{13}$ bis(4-chlorophenyl)acetylene, ${ }^{13} \quad$ bis(4bromophenyl)acetylene, ${ }^{13}$ bis(4-(trifluoromethyl)phenyl)acetylene, ${ }^{13}$ bis(3-chlorophenyl)acetylene, ${ }^{13}$ and bis(4-methylphenyl)acetylene ${ }^{13}$ were prepared according to the published procedures. All other reagents were purchased in the highest purity from commercial suppliers and used as received.

Vials used in the glovebox were dried in a gravity oven at $140^{\circ} \mathrm{C}$ for a minimum of $12 \mathrm{~h}$, transferred into the glovebox hot, and then stored at rt in the glovebox prior to use. All hot glassware was oven dried for a minimum of four hours or flame-dried under vacuum prior to use. "Double manifold" refers to a standard Schlenk-line gas manifold equipped with nitrogen and vacuum (ca. $0.100 \mathrm{~mm} \mathrm{Hg}$ ). All optimization reactions $(0.25 \mathrm{mmol})$ were charged in a nitrogen-filled glovebox and alkyl zinc halide was added on the bench via syringe then stirred on a magnetic stir plate. All yields in optimization reactions were determined using ${ }^{1} \mathrm{H}$ NMR with 1,3,5-trimethoxybenzene an internal standard and syn:anti ratios were determined using GC of unpurified products. All other reactions were set up using standard Schlenk technique and heated with stirring in temperature-controlled oil baths. Note: Any product yields listed in the main text that do not match those listed in the supporting information are the average of multiple isolated yields. The procedures listed below reflect yields from specific experimental runs. In this Supporting Information and in the main text, "dioxane" refers to 1,4-dioxane.

\section{Instrumentation and Chromatography:}

$400 \mathrm{MHz}{ }^{1} \mathrm{H}, 101 \mathrm{MHz}{ }^{13} \mathrm{C}$ and $376 \mathrm{MHz}{ }^{19} \mathrm{~F}$ NMR spectra were obtained on a $400 \mathrm{MHz}$ FT-NMR spectrometer equipped with a Bruker CryoPlatform. $600 \mathrm{MHz}{ }^{1} \mathrm{H}, 151 \mathrm{MHz}{ }^{13} \mathrm{C}, 119 \mathrm{MHz}{ }^{29} \mathrm{Si}, 565 \mathrm{MHz}$ ${ }^{19} \mathrm{~F}, 193 \mathrm{MHz}{ }^{11} \mathrm{~B}$, and $243 \mathrm{MHz}{ }^{31} \mathrm{P}$ spectra were obtained on a $600 \mathrm{MHz}$ FT-NMR spectrometer equipped with a Bruker SMART probe. All samples were analyzed in the indicated deutero-solvent and were recorded at ambient temperatures. All chemical shifts are reported in ppm. ${ }^{1} \mathrm{H}$ NMR spectra were calibrated using the residual protio-signal in deutero-solvents as a standard. ${ }^{13} \mathrm{C}$ NMR spectra were calibrated using the deutero-solvent as a standard. IR spectra were recorded on a Nicolet Magma-IR 560 FT-IR spectrometer as thin films on $\mathrm{KBr}$ plates. High resolution MS data was obtained on a Waters GCT Premier spectrometer using chemical ionization (CI), electron ionization (EI), or liquid injection field desorption ionization (LIFDI) or a Thermo Q-Exactive Orbitrap using electrospray ionization (ESI). Vacuum controller refers to J-Kem Digital Vacuum Regulator Model 200. Unless otherwise noted, column chromatography was performed either by hand or by use of Isolera 1 Biotage unit with 40-63 $\mu \mathrm{m}$ silica gel for normal phase and an Ultra $\mathrm{C} 18$ column for reverse phase. The eluent is reported in parentheses. Analytical thin-layer chromatography (TLC) was performed on silica gel (60 F254 Merck) pre-coated glass plates and visualized by UV or by staining with iodine, $\mathrm{KMnO}_{4}$, or cerium ammonium molybdate stain. 


\section{Synthesis of Alkynes}

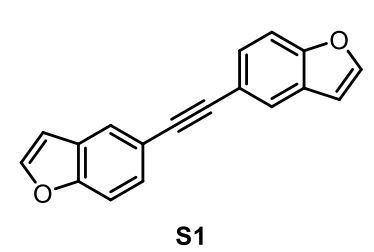

Chemical Formula: $\mathrm{C}_{18} \mathrm{H}_{10} \mathrm{O}_{2}$ Exact Mass: 258.0681

Molecular Weight: 258.2760

(S1) S1 was prepared through a modified version of the procedure described by Grieco ${ }^{13}$. An oven-dried $250 \mathrm{~mL}$ round bottom flask equipped with a magnetic stirbar and rubber septum was attached to a double manifold and cooled under vacuum. The septum was removed and the flask was charged with $\left(\mathrm{Ph}_{3} \mathrm{P}\right)_{2} \mathrm{PdCl}_{2}$ ( $840 \mathrm{mg}, 1.2 \mathrm{mmol}, 6 \mathrm{~mol} \%$ ) and Cul ( $380 \mathrm{mg}, 2.0 \mathrm{mmol}, 10 \mathrm{~mol} \%)$. The septum was then replaced and the flask was evacuated and refilled with nitrogen 3 times. Nitrogen-sparged benzene $(100 \mathrm{~mL})$ and nitrogen-sparged DBU $(18 \mathrm{~mL}, 120$ mmol, 6 equiv) were added to the flask via cannula addition. 5-bromobenzofuran (2.5 $\mathrm{mL}, 20 \mathrm{mmol}, 1$ equiv) was added to the flask via syringe. In rapid succession, trimethylsilylacetylene $(1.38 \mathrm{~mL}, 10 \mathrm{mmol}, 0.5$ equiv), and nitrogen-sparged water (144 $\mu \mathrm{L}, 8.0 \mathrm{mmol}, 0.4$ equiv) were added to the flask via syringe. The flask allowed to stir at $80^{\circ} \mathrm{C}$ in an oil bath for $48 \mathrm{~h}$; at the end of which, the flask was removed from the heat and cooled to rt. The reaction mixture was opened to air and transferred to a separatory funnel containing diethyl ether $(50 \mathrm{~mL})$ and water $(25 \mathrm{~mL})$. The partitioned solution was washed with $2 \mathrm{M}$ aqueous hydrogen chloride solution ( $3 \times 50 \mathrm{~mL})$, water $(25 \mathrm{~mL})$, saturated aqueous sodium chloride solution $(30 \mathrm{~mL})$, dried over anhydrous magnesium sulfate, filtered, and concentrated in vacuo. The crude material was purified via flash column chromatography (1:99 to 5:95 ethyl acetate : hexanes) to provide $\mathbf{S} 1$ as a pale yellow crystalline solid (1.4 g, $54 \%$ ).

${ }^{1} \mathrm{H}$ NMR $\left(400 \mathrm{MHz}, \mathrm{CDCl}_{3}\right) \delta 7.82(\mathrm{~s}, 2 \mathrm{H}), 7.66(\mathrm{~d}, \mathrm{~J}=2.2 \mathrm{~Hz}, 2 \mathrm{H}), 7.55-7.45(\mathrm{~m}, 4 \mathrm{H}), 6.78(\mathrm{~d}, \mathrm{~J}=2.2$ $\mathrm{Hz}, 2 \mathrm{H})$.

${ }^{13} \mathrm{C}$ NMR $\left(101 \mathrm{MHz}, \mathrm{CDCl}_{3}\right) \delta 154.6,145.9,128.1,127.7,124.7,118.1,111.7,106.6,88.4$.

FTIR $\left(\mathrm{cm}^{-1}\right): 3774,1469,1105,1026$.

$m p=171-172{ }^{\circ} \mathrm{C}$.

HRMS (LIFDI) m/z, calcd for [ $\left.\mathrm{C}_{18} \mathrm{H}_{10} \mathrm{O}_{2}\right]^{+}: 258.0681$; found: 258.0687. 


\section{Synthesis of Alkylzinc Halides}

\section{General Procedure A:}

An oven dried Schlenk flask equipped with a magnetic stirbar and rubber septum was attached to a double manifold and cooled under vacuum. The flask was backfilled with $\mathrm{N}_{2}$, the septum removed, and zinc dust ( 2 equiv) was added. The septum was replaced and the flask was attached to a double manifold and evacuated. Under vacuum, the zinc was heated for 5 minutes with a heat gun then allowed to cool to room temperature under vacuum. The flask was then backfilled with $\mathrm{N}_{2}$ and dioxane [2 $\left.\mathrm{M}\right]$ and 1,2-dibromoethane ( 0.03 equiv) were added. The flask was heated until reflux with a heat gun then allowed to cool. The process was repeated twice and after the flask was cooled to room temperature. Trimethylsilyl chloride ( 0.03 equiv) was added and the flask was stirred for 15 mins before the alkyl iodide (1 equiv) was added. The flask was then stirred in an oil bath at $100{ }^{\circ} \mathrm{C}$ for the indicated time. Conversion of starting halide was monitored via $\mathrm{GC}$ by quenching reaction aliquots with saturated $\mathrm{NH}_{4} \mathrm{Cl}$ solution and extracting with $\mathrm{Et}_{2} \mathrm{O}$. Once all starting halide was consumed, the excess zinc was allowed to settle while the flask cooled. The mixture was cannula filtered to a Schlenk tube. If insoluble particles persisted, filtration through a $0.2 \mu \mathrm{m}$ PTFE syringe filter was employed. Solutions were then titrated with $\mathrm{I}_{2}$ according to the literature procedure by Knochel. ${ }^{14}$

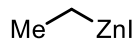

s2

Chemical Formula: $\mathrm{C}_{2} \mathrm{H}_{5} \mathrm{IZn}$

Exact Mass: 219.8727

Molecular Weight: 221.3465

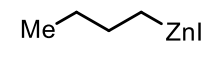

s3

Chemical Formula: $\mathrm{C}_{4} \mathrm{H}_{9} \mathrm{IZn}$

Exact Mass: 247.9040

Molecular Weight: 249.4005

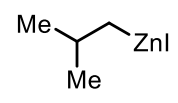

s4

Chemical Formula: $\mathrm{C}_{4} \mathrm{H}_{9} \mathrm{IZn}$

Exact Mass: 247.9040

Molecular Weight: 249.4005

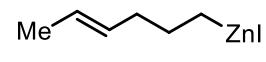

S5

Chemical Formula: $\mathrm{C}_{6} \mathrm{H}_{11} \mathrm{IZn}$ Exact Mass: 273.9197

Molecular Weight: 275.4385

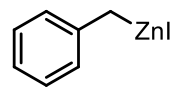

S6

Chemical Formula: $\mathrm{C}_{7} \mathrm{H}_{7} \mathrm{IZn}$

Exact Mass: 281.8884

Molecular Weight: 283.4175
(S2) According to general procedure A, $100 \mathrm{~mL}$ Schlenk flask was charged with zinc dust $(13 \mathrm{~g}, 200 \mathrm{mmol})$, dioxane $(40 \mathrm{~mL})$, trimethylsilyl chloride $(380 \mu \mathrm{L})$, and 1-iodoethane $(8.0 \mathrm{~mL}, 100 \mathrm{mmol})$. No 1,2-dibromoethane was added. The flask was heated to $100^{\circ} \mathrm{C}$ for $24 \mathrm{~h}$. Filtration and titration resulted in a [1.9 M] solution of ethylzinc iodide in dioxane.

(S3) According to general procedure $A, 100 \mathrm{~mL}$ Schlenk flask was charged with zinc dust (16 g, $250 \mathrm{mmol})$, dioxane $(40 \mathrm{~mL})$, trimethylsilyl chloride $(475 \mu \mathrm{L})$, and 1-iodobutane (15 mL, $125 \mathrm{mmol})$. No 1,2-dibromoethane was added. The flask was heated to $100^{\circ} \mathrm{C}$ for $24 \mathrm{~h}$. Filtration and titration resulted in a [2.0 M] solution of $n$ butylzinc iodide in dioxane.

(S4) According to general procedure A, $25 \mathrm{~mL}$ Schlenk flask was charged with zinc dust $(2.6 \mathrm{~g}, 40 \mathrm{mmol})$, dioxane $(10 \mathrm{~mL})$, trimethylsilyl chloride $(76 \mu \mathrm{L})$, and 1-iodo2-methylpropane $(1.9 \mathrm{~mL}, 20 \mathrm{mmol})$. No 1,2-dibromoethane was added. The flask was heated to $100^{\circ} \mathrm{C}$ for $24 \mathrm{~h}$. Filtration and titration resulted in a [1.0 M] solution of isobutylzinc iodide in dioxane.

(S5) According to general procedure A, $25 \mathrm{~mL}$ Schlenk flask was charged with zinc dust $(2.6 \mathrm{~g}, 40 \mathrm{mmol})$, dioxane $(10 \mathrm{~mL}), 1,2$-dibromoethane $(75 \mu \mathrm{L})$, trimethylsilyl chloride $(75 \mu \mathrm{L})$, and $(E)-6$-iodo-2-hexene $(4.2 \mathrm{~g}, 20 \mathrm{mmol})$. The flask was heated to $100{ }^{\circ} \mathrm{C}$ for $20 \mathrm{~h}$. Filtration and titration resulted in a [0.93 M] solution of $(E)-2-$ hexenezinc iodide in dioxane

(S6) According to general procedure A, $25 \mathrm{~mL}$ Schlenk flask was charged with zinc dust $(2.6 \mathrm{~g}, 40 \mathrm{mmol})$, dioxane $(10 \mathrm{~mL})$, trimethylsilyl chloride $(75 \mu \mathrm{L})$, and iodomethylbenzene $(4.3 \mathrm{~g}, 20 \mathrm{mmol})$. No 1,2-dibromoethane was added. The flask was heated to $100^{\circ} \mathrm{C}$ for $24 \mathrm{~h}$. Filtration and titration resulted in a [0.70 M] solution of benzylzinc iodide in dioxane. 

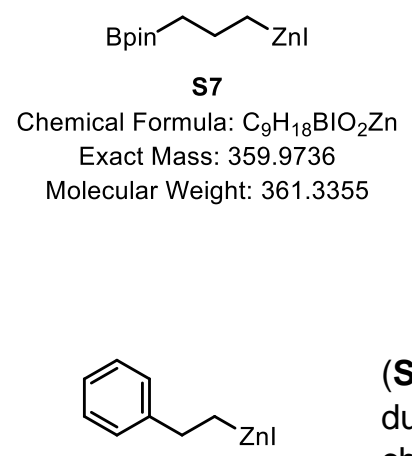

S8

Chemical Formula: $\mathrm{C}_{8} \mathrm{H}_{9} \mathrm{IZn}$

Exact Mass: 295.9040

Molecular Weight: 297.4445

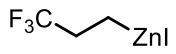

s9

Chemical Formula: $\mathrm{C}_{3} \mathrm{H}_{4} \mathrm{~F}_{3} \mathrm{IZn}$

Exact Mass: 287.8601

Molecular Weight: 289.3447
(S7) According to general procedure A, $50 \mathrm{~mL}$ Schlenk flask was charged with zinc dust $(2.1 \mathrm{~g}, 32 \mathrm{mmol})$, dioxane $(15 \mathrm{~mL}), 1,2$-dibromoethane $(100 \mu \mathrm{L})$, trimethylsilyl chloride (100 $\mu \mathrm{L}$ ), and 2-(3-iodopropyl)-4,4,5,5-tetramethyl-1,3,2dioxaborolane $(4.9 \mathrm{~g}, 16 \mathrm{mmol})$. The flask was heated to $100{ }^{\circ} \mathrm{C}$ for $24 \mathrm{~h}$. Filtration and titration resulted in a [1.0 M] solution of 3-(4,4,5,5,-tetramethyl1,3,2-dioxaborolan-2-yl)zinc iodide in dioxane.

(S8) According to general procedure A, $25 \mathrm{~mL}$ Schlenk flask was charged with zinc dust $(3.9 \mathrm{~g}, 60 \mathrm{mmol})$, dioxane $(15 \mathrm{~mL}), 1,2$-dibromoethane $(100 \mu \mathrm{L})$, trimethylsilyl chloride $(100 \mu \mathrm{L})$, and (2-iodoethyl)-benzene $(4.3 \mathrm{~mL}, 30 \mathrm{mmol})$. The flask was heated to $100{ }^{\circ} \mathrm{C}$ for $24 \mathrm{~h}$. Filtration and titration resulted in a [1.2 M] solution of phenethylzinc iodide in dioxane.

(S9) According to general procedure A, $50 \mathrm{~mL}$ Schlenk flask was charged with zinc dust $(3.9 \mathrm{~g}, 60 \mathrm{mmol})$, dioxane $(20 \mathrm{~mL})$, 1,2-dibromoethane $(155 \mu \mathrm{L})$, trimethylsilyl chloride $(113 \mu \mathrm{L})$, and 1-iodo-3,3,3-trifluoropropane $(3.5 \mathrm{~mL}, 30$ $\mathrm{mmol}$ ). The flask was heated to $100^{\circ} \mathrm{C}$ for $24 \mathrm{~h}$. Filtration and titration resulted in a [1.24 M] solution of (3,3,3-trifluoropropyl)zinc iodide in dioxane.

\section{Synthesis of Tetrasubstituted Vinylsilanes via Multicomponent Carbosilylation Reactions}

\section{General Procedure B: Aryl Alkynes}

Note: THF was used to quench certain reactions to avoid disiloxane formation upon aqueous workup. This quench generates the more easily separated (4-iodobutoxy)silane through silyl-iodide induced ring opening of THF.

A $25 \mathrm{~mL}$ Schlenk flask equipped with a magnetic stirbar and rubber septum was flame-dried, allowed to cool to room temperature under vacuum, and refilled with $\mathrm{N}_{2}$. The flask was briefly opened, charged with the given alkyne ( 1 equiv) and palladium precatalyst ( 0.02 equiv), and the septum was replaced. The flask was evacuated and refilled with nitrogen 3 times. Dioxane, triethylamine ( 3 equiv), and silyl-iodide ( 3 equiv) were added sequentially via syringe at room temperature with stirring. A solution of alkylzinc iodide in dioxane (1.5 equiv) was then added dropwise over $4 \mathrm{~h}$ via syringe pump. The reaction was allowed to stir at room temperature for $0.25 \mathrm{~h}$ after the addition was completed. The reaction was quenched as indicated and opened to air. The resultant mixture was transferred to a separatory funnel and partitioned between 1 $\mathrm{M}$ aqueous hydrogen chloride solution $(3 \mathrm{~mL})$ to solubilize the zinc salts, water $(10 \mathrm{~mL})$ and diethyl ether $(20 \mathrm{~mL})$. The aqueous layer was extracted with diethyl ether $(3 \times 15 \mathrm{~mL})$. The combined organic layers were washed with saturated aqueous sodium chloride solution $(30 \mathrm{~mL})$, dried over anhydrous magnesium sulfate, filtered, and concentrated in vacuo. A small aliquot of the crude reaction mixture $(\sim 20 \mu \mathrm{L})$ was analyzed by gas chromatography or NMR spectroscopy to determine the syn:anti ratio. The crude material was purified via silica column chromatography in the given solvent combination, followed by reverse phase column chromatography on a Biotage instrument in the given solvent combination. 


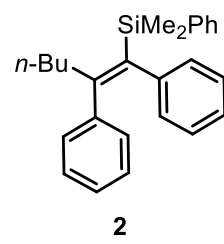

Chemical Formula: $\mathrm{C}_{26} \mathrm{H}_{30} \mathrm{Si}$ Exact Mass: 370.2117 Molecular Weight: 370.6110

(2) According to the general procedure $\mathrm{B},\left(\mathrm{Ph}_{3} \mathrm{P}\right)_{2} \mathrm{PdCl}_{2}(28 \mathrm{mg}, 0.04 \mathrm{mmol}, 2 \mathrm{~mol}$ $\%$ ), diphenyl acetylene (357 mg, $2.0 \mathrm{mmol}, 1$ equiv), dioxane (1 mL, $2.0[\mathrm{M}])$, triethylamine ( $836 \mu \mathrm{L}, 6.0 \mathrm{mmol}, 3$ equiv), and dimethylphenylsilyl iodide $(1.12 \mathrm{~mL}$, $6.0 \mathrm{mmol}, 3$ equiv) were combined under nitrogen. $n$-Butylzinc iodide $\mathbf{S} 3$ in dioxane ([1.5 M], $2.0 \mathrm{~mL}, 3.0 \mathrm{mmol}, 1.5$ equiv) was added over $4 \mathrm{~h}$. The reaction was quenched with water, stirred for $0.25 \mathrm{~h}$, and worked up according to the general procedure. Analysis of the crude reaction mixture via gas chromatography revealed a >95:5 syn:anti ratio. The product was purified by silica gel chromatography ( $1: 99$ to $3: 47$ ethyl acetate : hexanes) followed by reverse phase column chromatography on a Biotage instrument using a SNAP Ultra C18 $60 \mathrm{~g}$ column (7:13 to $0: 100$ water : acetonitrile) to afford 2 as a clear colorless oil (702 mg, 95\%).

${ }^{1} \mathrm{H} \mathrm{NMR}\left(600 \mathrm{MHz}, \mathrm{CDCl}_{3}\right) \delta 7.75-7.64(\mathrm{~m}, 2 \mathrm{H}), 7.44-7.36(\mathrm{~m}, 3 \mathrm{H}), 7.04(\mathrm{q}, J=7.8 \mathrm{~Hz}, 4 \mathrm{H}), 6.98(\mathrm{t}, J=$ $7.3 \mathrm{~Hz}, 1 \mathrm{H}), 6.92(\mathrm{dd}, J=11.7,7.3 \mathrm{~Hz}, 3 \mathrm{H}), 6.81(\mathrm{~d}, J=7.1 \mathrm{~Hz}, 2 \mathrm{H}), 2.50-2.37(\mathrm{~m}, 2 \mathrm{H}), 1.16-0.91(\mathrm{~m}$, $4 \mathrm{H}), 0.65$ (t, $J=7.1 \mathrm{~Hz}, 3 \mathrm{H}), 0.28(\mathrm{~s}, 6 \mathrm{H})$.

${ }^{13} \mathrm{C}$ NMR $\left(151 \mathrm{MHz}, \mathrm{CDCl}_{3}\right) \delta 156.2,144.6,143.2,140.3,138.2,133.9,129.4,128.9,128.8,128.0,127.4$, $127.3,125.8,124.6,38.7,30.7,22.8,14.0,0.0$.

${ }^{29} \mathrm{Si} \mathrm{NMR}\left(119 \mathrm{MHz}, \mathrm{CDCl}_{3}\right) \delta-11.6$.

FTIR $\left(\mathrm{cm}^{-1}\right):$ 3067, 3019, 2956, 2859, 1587, 1486, 1427, 1249, 1112, 834, 809, 699.

HRMS (LIFDI) m/z, calcd for [ $\mathrm{C}_{26} \mathrm{H}_{30} \mathrm{Si}^{+}:$: 370.2117; found: 370.2108 .

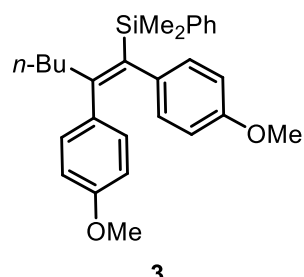

Chemical Formula: $\mathrm{C}_{28} \mathrm{H}_{34} \mathrm{O}_{2} \mathrm{Si}$

Exact Mass: 430.2328

Molecular Weight: 430.6630
(3) According to the general procedure $\mathrm{B}\left(\mathrm{Ph}_{3} \mathrm{P}\right)_{2} \mathrm{PdCl}_{2}(28 \mathrm{mg}, 0.04 \mathrm{mmol}, 2 \mathrm{~mol}$ $\%$ ), 1,2-bis(4-methoxyphenyl)acetylene (476 mg, $2.0 \mathrm{mmol}$, 1 equiv), dioxane (1 $\mathrm{mL}, 2.0[\mathrm{M}])$, triethylamine ( $836 \mu \mathrm{L}, 6.0 \mathrm{mmol}, 3$ equiv), and dimethylphenylsilyl iodide ( $1.12 \mathrm{~mL}, 6.0 \mathrm{mmol}, 3$ equiv) were combined under nitrogen. $n$-Butylzinc iodide S3 in dioxane ([1.9 M], $1.6 \mathrm{~mL}, 3.0 \mathrm{mmol}, 1.5$ equiv) was added over $4 \mathrm{~h}$. The reaction was quenched with water, stirred for $0.25 \mathrm{~h}$, and worked up according to the general procedure. Analysis of the crude reaction mixture via gas chromatography revealed a 91:9 syn:anti ratio. The product was purified by silica gel chromatography (1:99 to $3: 47$ ethyl acetate : hexanes) followed by reverse phase column chromatography on a Biotage instrument using a SNAP Ultra C18 60 g column (1:1 to $0: 100$ water : acetonitrile) to afford 3 as a clear colorless oil (692 $\mathrm{mg}, 81 \%$ ). $6.88(\mathrm{~d}, J=8.7 \mathrm{~Hz}, 2 \mathrm{H}), 6.82(\mathrm{~d}, J=8.7 \mathrm{~Hz}, 2 \mathrm{H}), 6.72(\mathrm{~d}, J=8.6 \mathrm{~Hz}, 2 \mathrm{H}), 3.81(\mathrm{~s}, 3 \mathrm{H}), 3.80(\mathrm{~s}, 3 \mathrm{H}), 2.22$ $-2.00(\mathrm{~m}, 2 \mathrm{H}), 1.13-0.96(\mathrm{~m}, 4 \mathrm{H}), 0.64(\mathrm{t}, J=7.1 \mathrm{~Hz}, 3 \mathrm{H}),-0.14(\mathrm{~s}, 6 \mathrm{H})$.

${ }^{13} \mathrm{C}$ NMR $\left(151 \mathrm{MHz}, \mathrm{CDCl}_{3}\right) \delta 158.6,157.4,156.1,140.6,139.3,136.8,136.5,134.0,130.1,129.7,128.3$, $127.4,113.4,113.1,55.4,55.3,37.1,30.4,22.6,14.0,-0.8$.

${ }^{29} \mathrm{Si} \operatorname{NMR}\left(119 \mathrm{MHz}, \mathrm{CDCl}_{3}\right) \delta-12.7$.

FTIR $\left(\mathrm{cm}^{-1}\right):$ 2954, 2831, 2361, 1607, 1508, 1242, 1173, 1108, 1037, 834, 811, 701.

HRMS (LIFDI) m/z, calcd for [ $\left.\mathrm{C}_{28} \mathrm{H}_{34} \mathrm{O}_{2} \mathrm{Si}\right]^{+}: 430.2328$; found: 430.2325. 


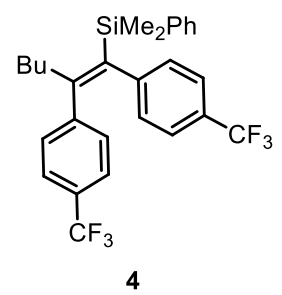

Chemical Formula: $\mathrm{C}_{28} \mathrm{H}_{28} \mathrm{~F}_{6} \mathrm{Si}$

Exact Mass: 506.1864

Molecular Weight: 506.6074

(4) According to the general procedure $\mathrm{B},\left[\left(\mathrm{C}_{6} \mathrm{~F}_{5}\right) \mathrm{P}\right]_{2} \mathrm{PdCl}_{2}(51 \mathrm{mg}, 0.04 \mathrm{mmol}, 2$ $\mathrm{mol} \%$ ), 1,2-bis(4-(trifluoromethyl)phenyl)acetylene (628 mg, $2.0 \mathrm{mmol}, 1$ equiv), dioxane (1 mL, $2.0[\mathrm{M}])$, triethylamine $(836 \mu \mathrm{L}, 6.0 \mathrm{mmol}, 3$ equiv), and dimethylphenylsilyl iodide (1.12 $\mathrm{mL}, 6.0 \mathrm{mmol}, 3$ equiv) were combined under nitrogen. $n$-Butylzinc iodide $\mathbf{S} 3$ in dioxane $([2.0 \mathrm{M}], 1.6 \mathrm{~mL}, 3.0 \mathrm{mmol}, 1.5$ equiv) was added over $4 \mathrm{~h}$. The reaction was quenched with water, stirred for $0.25 \mathrm{~h}$, and worked up according to the general procedure. Analysis of the crude reaction mixture via gas chromatography revealed a $>95: 5$ syn:anti ratio. The product was purified by silica gel chromatography (1:99 to $3: 47$ ethyl acetate : hexanes) followed by reverse phase column chromatography on a Biotage instrument using a SNAP Ultra C18 $60 \mathrm{~g}$ column ( $7: 13$ to $0: 100$ water : acetonitrile) to afford 4 as a white solid (866 $\mathrm{mg}, 85 \%)$.

${ }^{1} \mathrm{H}$ NMR $\left(600 \mathrm{MHz}, \mathrm{CDCl}_{3}\right) \delta 7.76-7.68(\mathrm{~m}, 2 \mathrm{H}), 7.56-7.45(\mathrm{~m}, 3 \mathrm{H}), 7.40(\mathrm{dd}, J=10.2,8.4 \mathrm{~Hz}, 4 \mathrm{H}), 7.07$ $(\mathrm{d}, J=8.1 \mathrm{~Hz}, 2 \mathrm{H}), 6.98(\mathrm{~d}, J=8.0 \mathrm{~Hz}, 2 \mathrm{H}), 2.52(\mathrm{t}, J=7.5 \mathrm{~Hz}, 2 \mathrm{H}), 1.18-1.00(\mathrm{~m}, 4 \mathrm{H}), 0.74(\mathrm{t}, J=6.8$ $\mathrm{Hz}, 3 \mathrm{H}), 0.38(\mathrm{~s}, 6 \mathrm{H})$.

${ }^{13} \mathrm{C}$ NMR (151 MHz, CDCl 3 ) 155.5, 148.0 (q, $\left.J=1.0 \mathrm{~Hz}\right), 146.3$ (q, $\left.J=1.0 \mathrm{~Hz}\right), 139.1,139.0,133.8$, 129.3, 129.3, 128.9, 128.2, 127.2 (q, $J=32.3 \mathrm{~Hz}), 127.2$ (q, $J=32.3 \mathrm{~Hz}), 124.7$ (q, $J=3.0 \mathrm{~Hz}), 124.6$ $(q, J=3.4 \mathrm{~Hz}), 124.4(q, J=271.8 \mathrm{~Hz}), 124.2(q, J=271.7 \mathrm{~Hz}), 38.4,30.5,22.8,13.9,-0.3$.

$\left.{ }^{29} \mathrm{Si} \mathrm{NMR} \mathrm{(119} \mathrm{MHz,} \mathrm{CDCl}_{3}\right) \delta-11.2$.

${ }^{19} \mathrm{~F}$ NMR $\left(565 \mathrm{MHz}, \mathrm{CDCl}_{3}\right) \delta-62.3,-62.5$.

FTIR $\left(\mathrm{cm}^{-1}\right)$ : 3070, 2959, 2931, 2861, 1614, 1326, 1165, 1124, 1068, 1018, 845, 815, 701.

$\mathrm{mp}=46-48^{\circ} \mathrm{C}$.

HRMS (LIFDI) m/z, calcd for [ $\mathrm{C}_{28} \mathrm{H}_{28} \mathrm{~F}_{6} \mathrm{Si}^{+}$: 506.1864 ; found: 506.1866 .

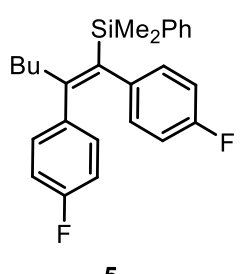

5

Chemical Formula: $\mathrm{C}_{26} \mathrm{H}_{28} \mathrm{~F}_{2} \mathrm{Si}$ Exact Mass: 406.1928 Molecular Weight: 406.5918
(5) According to the general procedure $\mathrm{B},\left(\mathrm{Ph}_{3} \mathrm{P}\right)_{2} \mathrm{PdCl}_{2}(28 \mathrm{mg}, 0.04 \mathrm{mmol}, 2$ mol \%), 1,2-bis(4-fluorophenyl)acetylene (428 mg, $2.0 \mathrm{mmol}, 1$ equiv), dioxane (1 mL, $2.0[\mathrm{M}])$, triethylamine ( $836 \mu \mathrm{L}, 6.0 \mathrm{mmol}, 3$ equiv), and dimethylphenylsilyl iodide ( $1.10 \mathrm{~mL}, 6.0 \mathrm{mmol}, 3$ equiv) were combined under nitrogen. $n$-Butylzinc iodide S3 in dioxane ([1.5 M], $2.0 \mathrm{~mL}, 3.0 \mathrm{mmol}, 1.5$ equiv) was added over $4 \mathrm{~h}$. The reaction was quenched with water, stirred for $0.25 \mathrm{~h}$, and worked up according to the general procedure. Analysis of the crude reaction mixture via gas chromatography revealed a $>95: 5$ syn:anti ratio. The product was purified by silica gel chromatography ( $1: 99$ to $3: 47$ ethyl acetate : hexanes) followed by reverse phase column chromatography on a Biotage instrument using a SNAP Ultra C18 $60 \mathrm{~g}$ column (7:13 to $0: 100$ water : acetonitrile) to afford 5 as a white solid (571 $\mathrm{mg}, 70 \%)$.

${ }^{1} \mathrm{H}$ NMR $\left(600 \mathrm{MHz}, \mathrm{CDCl}_{3}\right) \delta 7.67-7.59(\mathrm{~m}, 2 \mathrm{H}), 7.49-7.33(\mathrm{~m}, 3 \mathrm{H}), 6.84(\mathrm{dd}, J=8.0,5.9 \mathrm{~Hz}, 2 \mathrm{H}), 6.82$ $-6.64(\mathrm{~m}, 6 \mathrm{H}), 2.50-2.22(\mathrm{~m}, 2 \mathrm{H}), 1.11-0.85(\mathrm{~m}, 4 \mathrm{H}), 0.65(\mathrm{t}, J=6.7 \mathrm{~Hz}, 3 \mathrm{H}), 0.28(\mathrm{~s}, 6 \mathrm{H})$.

${ }^{13} \mathrm{C}$ NMR $\left(151 \mathrm{MHz}, \mathrm{CDCl}_{3}\right) \delta 161.6(\mathrm{~d}, J=76.9 \mathrm{~Hz}), 160.0(\mathrm{~d}, J=75.5 \mathrm{~Hz}), 155.9,140.3(\mathrm{~d}, J=3.2 \mathrm{~Hz})$, 139.8, 138.8 (d, $J=3.2 \mathrm{~Hz}$ ), 138.0, 133.8, $130.6(\mathrm{~d}, J=7.7 \mathrm{~Hz}), 130.2(\mathrm{~d}, J=7.8 \mathrm{~Hz}), 129.1,128.0,114.5$ $(\mathrm{d}, J=6.4 \mathrm{~Hz}), 114.4(\mathrm{~d}, J=6.4 \mathrm{~Hz}), 38.6,30.7,22.8,14.0,-0.1$.

${ }^{29} \mathrm{Si} \mathrm{NMR}\left(119 \mathrm{MHz}, \mathrm{CDCl}_{3}\right) \delta-11.5$.

${ }^{19} \mathrm{~F} \mathrm{NMR}\left(565 \mathrm{MHz}, \mathrm{CDCl}_{3}\right) \delta-116.7,-118.7$. 
FTIR $\left(\mathrm{cm}^{-1}\right):$ 3068, 2957, 2929, 2860, 1504, 1221, 1157, 834, 816, 701.

$\mathrm{mp}=55-56^{\circ} \mathrm{C}$.

HRMS (LIFDI) m/z, calcd for [ $\mathrm{C}_{26} \mathrm{H}_{28} \mathrm{~F}_{2} \mathrm{Si}^{+}$: 406.1928 ; found: 406.1932 .

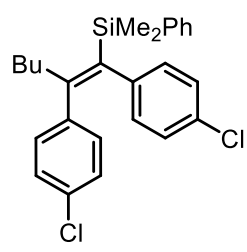

6

Chemical Formula: $\mathrm{C}_{26} \mathrm{H}_{28} \mathrm{Cl}_{2} \mathrm{Si}$

Exact Mass: 438.1337

Molecular Weight: 439.4950

(6) According to the general procedure $\mathrm{B},\left(\mathrm{Ph}_{3} \mathrm{P}\right)_{2} \mathrm{PdCl}_{2}(28 \mathrm{mg}, 0.04 \mathrm{mmol}, 2$ mol \%), 1,2-bis(4-chlorophenyl)acetylene (552 mg, 2 mmol, 1 equiv), dioxane (20 mL, 0.1 [M]), triethylamine (836 $\mu \mathrm{L}, 6 \mathrm{mmol}, 3$ equiv), and dimethylphenylsilyl iodide (1.12 mL, $6 \mathrm{mmol}, 3$ equiv) were combined under nitrogen. $n$-Butylzinc iodide S3 in dioxane ([1.9 M], $1.57 \mathrm{~mL}, 3 \mathrm{mmol}, 1.5$ equiv) was added over $4 \mathrm{~h}$. The reaction was quenched with THF, stirred for $0.25 \mathrm{~h}$, and worked up according to the general procedure. Analysis of the crude reaction mixture via gas chromatography revealed a $>95: 5$ syn:anti ratio. The product was purified by silica gel chromatography (hexanes) followed by reverse phase column chromatography on a Biotage instrument using a SNAP Ultra C18 60 g column ( $3: 17$ water : acetonitrile) to afford 6 as a white crystalline solid (542 mg, 62\%).

${ }^{1} \mathrm{H}$ NMR $\left(400 \mathrm{MHz}, \mathrm{CDCl}_{3}\right) \delta 7.66-7.59(\mathrm{~m}, 2 \mathrm{H}), 7.42-7.35(\mathrm{~m}, 3 \mathrm{H}), 7.04(\mathrm{~d}, J=8.3 \mathrm{~Hz}, 4 \mathrm{H}), 6.81$ (d, J $=8.3 \mathrm{~Hz}, 2 \mathrm{H}), 6.71(\mathrm{~d}, J=8.3 \mathrm{~Hz}, 2 \mathrm{H}), 2.38(\mathrm{t}, J=7.5 \mathrm{~Hz}, 2 \mathrm{H}), 0.99(\mathrm{dt}, J=7.5,3.7 \mathrm{~Hz}, 4 \mathrm{H}), 0.68-0.60$ $(\mathrm{m}, 3 \mathrm{H}), 0.27(\mathrm{~s}, 6 \mathrm{H})$.

${ }^{13} \mathrm{C} \mathrm{NMR}\left(101 \mathrm{MHz}, \mathrm{CDCl}_{3}\right) \delta 155.6,142.8,141.2,139.6,138.1,133.8,131.8,130.6,130.5,130.0,129.1$, $128.1,127.9,127.8,38.5,30.6,22.8,14.0,-0.1$.

${ }^{29} \mathrm{Si} \mathrm{NMR}\left(119 \mathrm{MHz}, \mathrm{CDCl}_{3}\right) \delta-11.5$.

FTIR $\left(\mathrm{cm}^{-1}\right): 2956,2928,1486,1250,1111,1090,1014,836,811,774,700,492$.

$\mathrm{mp}=60-61^{\circ} \mathrm{C}$.

HRMS (LIFDI) m/z, calcd for [ $\left.\mathrm{C}_{26} \mathrm{H}_{28} \mathrm{SiCl}_{2}\right]^{+}: 438.1337$; found: 438.1315.

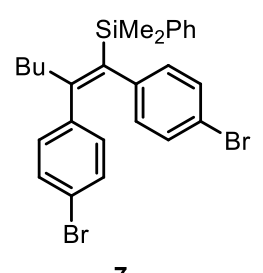

Chemical Formula: $\mathrm{C}_{26} \mathrm{H}_{28} \mathrm{Br}_{2} \mathrm{Si}$

Exact Mass: 526.0327

Molecular Weight: 528.4030

(7) According to the general procedure $\mathrm{B},\left(\mathrm{Ph}_{3} \mathrm{P}\right)_{2} \mathrm{PdCl}_{2}(14 \mathrm{mg}, 0.02 \mathrm{mmol}, 2$ mol \%), 1,2-bis(4-bromophenyl)acetylene (314 mg, $1 \mathrm{mmol}, 1$ equiv), dioxane (10 mL, 0.1 [M]), triethylamine $(418 \mu \mathrm{L}, 3 \mathrm{mmol}, 3$ equiv), and dimethylphenylsilyl iodide (560 $\mu \mathrm{L}, 3 \mathrm{mmol}, 3$ equiv) were combined under nitrogen. $n$-Butylzinc iodide S3 in dioxane ([1.9 M], $790 \mu \mathrm{L}, 1.5 \mathrm{mmol}, 1.5$ equiv) was added over $4 \mathrm{~h}$. The reaction was quenched with THF, stirred for $0.25 \mathrm{~h}$, and worked up according to the general procedure. Analysis of the crude reaction mixture via gas chromatography revealed a $>95: 5$ syn:anti ratio. The product was purified by silica gel chromatography (1:99 ethyl acetate : hexanes) followed by reverse phase column chromatography on a Biotage instrument using a SNAP Ultra C18 $60 \mathrm{~g}$ column ( $3: 17$ water : acetonitrile) to afford 7 as a clear pale yellow oil ( $335 \mathrm{mg}, 63 \%$ ).

${ }^{1} \mathrm{H}$ NMR $\left(400 \mathrm{MHz}, \mathrm{CDCl}_{3}\right) \delta 7.64(\mathrm{dd}, J=6.5,2.9 \mathrm{~Hz}, 2 \mathrm{H}), 7.41-7.39(\mathrm{~m}, 3 \mathrm{H}), 7.21$ (dd, $J=8.3,6.4 \mathrm{~Hz}$, $4 \mathrm{H}), 6.78(\mathrm{~d}, J=8.4 \mathrm{~Hz}, 2 \mathrm{H}), 6.68(\mathrm{~d}, J=8.3 \mathrm{~Hz}, 2 \mathrm{H}), 2.40(\mathrm{t}, J=7.5 \mathrm{~Hz}, 2 \mathrm{H}), 1.02-0.98(\mathrm{~m}, 4 \mathrm{H}), 0.65(\mathrm{t}$, $J=6.9 \mathrm{~Hz}, 3 \mathrm{H}), 0.29(\mathrm{~s}, 6 \mathrm{H})$.

${ }^{13} \mathrm{C} \mathrm{NMR}\left(151 \mathrm{MHz}, \mathrm{CDCl}_{3}\right) \delta 155.5,143.3,141.7,139.5,138.2,133.8,130.9,130.8,130.8,130.4,129.1$, $128.1,120.1,118.9,38.4,30.6,22.7,13.9,-0.1$.

$\left.{ }^{29} \mathrm{Si} \mathrm{NMR} \mathrm{(119} \mathrm{MHz,} \mathrm{CDCl}_{3}\right) \delta-11.5$. 
FTIR (cm-1): 2956, 2928, 1483, 1249, 1110, 1072, 1010, 835, 812, 796, 732, 701, 515.

HRMS (LIFDI) m/z, calcd for [ $\left.\mathrm{C}_{26} \mathrm{H}_{28} \mathrm{Br}_{2} \mathrm{Si}\right]^{+}: 526.0327$; found: 526.0328 .

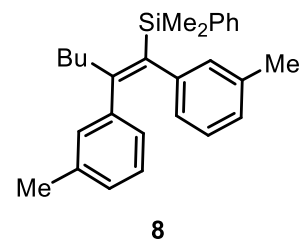

Chemical Formula: $\mathrm{C}_{28} \mathrm{H}_{34} \mathrm{Si}$ Exact Mass: 398.2430 Molecular Weight: 398.6650

(8) According to the general procedure $\mathrm{B},\left(\mathrm{Ph}_{3} \mathrm{P}\right)_{2} \mathrm{PdCl}_{2}(28 \mathrm{mg}, 0.04 \mathrm{mmol}, 2 \mathrm{~mol}$ $\%$ ), 1,2-bis(3-methylphenyl)acetylene (413 mg, $2.0 \mathrm{mmol}$, 1 equiv), dioxane ( $1 \mathrm{~mL}$, $2.0[\mathrm{M}])$, triethylamine $(836 \mu \mathrm{L}, 6.0 \mathrm{mmol}, 3$ equiv), and dimethylphenylsilyl iodide $(1.12 \mathrm{~mL}, 6.0 \mathrm{mmol}, 3$ equiv) were combined under nitrogen. $n$-Butylzinc iodide S3 in dioxane ([1.9 M], $1.6 \mathrm{~mL}, 3.0 \mathrm{mmol}, 1.5$ equiv) was added over $4 \mathrm{~h}$. The reaction was quenched with water, stirred for $0.25 \mathrm{~h}$, and worked up according to the general procedure. Analysis of the crude reaction mixture via gas chromatography revealed a >95:5 syn:anti ratio. The product was purified by silica gel chromatography ( $1: 99$ to $3: 47$ ethyl acetate : hexanes) followed by reverse phase column chromatography on a Biotage instrument using a SNAP Ultra C18 $60 \mathrm{~g}$ column (7:13 to $0: 100$ water : acetonitrile) to afford 8 as a clear colorless oil $(621 \mathrm{mg}, 81 \%)$.

${ }^{1} \mathrm{H}$ NMR (600 MHz, $\left.\mathrm{CDCl}_{3}\right) \delta 7.69$ (dd, $\left.J=7.4,1.8 \mathrm{~Hz}, 2 \mathrm{H}\right), 7.47-7.34(\mathrm{~m}, 3 \mathrm{H}), 6.92(\mathrm{q}, J=7.6 \mathrm{~Hz}, 2 \mathrm{H})$, $6.79(\mathrm{~d}, J=7.5 \mathrm{~Hz}, 2 \mathrm{H}), 6.77-6.66(\mathrm{~m}, 3 \mathrm{H}), 6.66-6.55(\mathrm{~m}, 1 \mathrm{H}), 2.42-2.34(\mathrm{~m}, 2 \mathrm{H}), 2.17(\mathrm{~s}, 6 \mathrm{H}), 1.15-$ $0.86(\mathrm{~m}, 4 \mathrm{H}), 0.68-0.62(\mathrm{~m}, 3 \mathrm{H}), 0.27(\mathrm{~s}, 6 \mathrm{H})$.

${ }^{13} \mathrm{C}$ NMR $\left(151 \mathrm{MHz}, \mathrm{CDCl}_{3}\right) \delta 156.0,144.5,143.1,140.5,137.9,136.5,133.9,130.2,129.5,128.8,127.9$, $127.2,127.0,126.5,126.4,125.9,125.2,38.7,30.8,22.9,21.5,21.5,14.0,0.0$.

$\left.{ }^{29} \mathrm{Si} \mathrm{NMR} \mathrm{(119} \mathrm{MHz,} \mathrm{CDCl}_{3}\right) \delta-11.8$.

${ }^{1} \mathrm{H}$ NMR $\left(400 \mathrm{MHz}, \mathrm{C}_{6} \mathrm{D}_{6}\right) \delta 7.81(\mathrm{dd}, J=8.0,1.4 \mathrm{~Hz}, 2 \mathrm{H}), 7.36-7.29(\mathrm{~m}, 3 \mathrm{H}), 7.28-7.21(\mathrm{~m}, 1 \mathrm{H}), 7.02-$ $6.93(\mathrm{~m}, 1 \mathrm{H}), 6.94-6.78(\mathrm{~m}, 5 \mathrm{H}), 6.76-6.62(\mathrm{~m}, 2 \mathrm{H}), 2.66-2.50(\mathrm{~m}, 1 \mathrm{H}), 2.01(\mathrm{~d}, J=13.5 \mathrm{~Hz}, 6 \mathrm{H}), 1.24$ (p, $J=7.7 \mathrm{~Hz}, 2 \mathrm{H}), 1.02(\mathrm{~h}, J=7.3 \mathrm{~Hz}, 2 \mathrm{H}), 0.65(\mathrm{t}, J=7.3 \mathrm{~Hz}, 1 \mathrm{H}), 0.42(\mathrm{~s}, 6 \mathrm{H})$.

${ }^{13} \mathrm{C}$ NMR $\left(101 \mathrm{MHz}, \mathrm{C}_{6} \mathrm{D}_{6}\right) \delta 156.4,144.9,143.3,140.6,138.4,137.0,136.9,134.2,134.2,130.3,129.6$, 129.2, 128.4, 127.8, 127.6, 127.1, 126.9, 126.4, 125.9, 39.1, 31.2, 23.1, 21.5, 21.4, 14.1, 0.2.

${ }^{29} \mathrm{Si} \mathrm{NMR}\left(119 \mathrm{MHz}, \mathrm{C}_{6} \mathrm{D} 6\right) \delta-11.8$.

FTIR (cm-1): 3049, 3009, 2955, 2925, 2859, 1600, 1483, 1427, 1248, 1112, 826, 780, 702, 469.

HRMS (LIFDI) m/z, calcd for [ $\left.\mathrm{C}_{28} \mathrm{H}_{34} \mathrm{Si}\right]^{+}:$398.2430; found: 398.2431 .
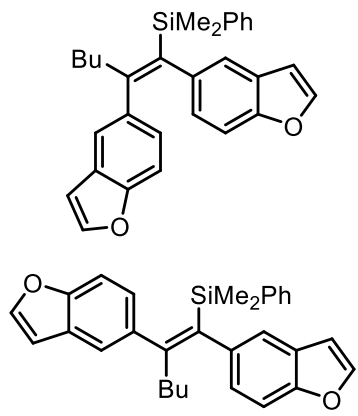

anti-9

Chemical Formula: $\mathrm{C}_{30} \mathrm{H}_{30} \mathrm{O}_{2} \mathrm{Si}$ Exact Mass: 450.2015 Molecular Weight: 450.6530
(9) According to the general procedure $\mathrm{B},\left(\mathrm{Ph}_{3} \mathrm{P}\right)_{2} \mathrm{PdCl}_{2}(28 \mathrm{mg}, 0.04 \mathrm{mmol}, 2$ mol \%), 1,2-bis(5-benzofuran)acetylene (516 mg, 2 mmol, 1 equiv), dioxane (20 $\mathrm{mL}, 0.1$ [M]), triethylamine (836 $\mu \mathrm{L}, 6 \mathrm{mmol}, 3$ equiv), and dimethylphenylsilyl iodide (1.12 mL, $6 \mathrm{mmol}, 3$ equiv) were combined under nitrogen. $n$-Butylzinc iodide S3 in dioxane ([1.9 M], $1.57 \mathrm{~mL}, 3 \mathrm{mmol}, 1.5$ equiv) was added over $4 \mathrm{~h}$. The reaction was quenched with THF, stirred for $0.25 \mathrm{~h}$, and worked up according to the general procedure. Analysis of the crude reaction mixture via gas chromatography revealed a 70:30 syn:anti ratio. The product was purified by silica gel chromatography (1:99 to $5: 95$ ethyl acetate : hexanes) followed by reverse phase column chromatography on a Biotage instrument using a SNAP Ultra $\mathrm{C} 1860 \mathrm{~g}$ column ( $3: 17$ water : acetonitrile) to afford the separable products syn-9 as a clear pale yellow oil ( $353 \mathrm{mg}, 40 \%$ ) and anti-9 as a clear pale yellow oil (130 mg, $15 \%)$. 


\section{Syn-9:}

${ }^{1} \mathrm{H} \mathrm{NMR}\left(400 \mathrm{MHz}, \mathrm{CDCl}_{3}\right) \delta 7.76-7.71(\mathrm{~m}, 2 \mathrm{H}), 7.45(\mathrm{t}, J=2.4 \mathrm{~Hz}, 2 \mathrm{H}), 7.42(\mathrm{dd}, J=5.9,1.2 \mathrm{~Hz}, 3 \mathrm{H})$, $7.19(\mathrm{~d}, J=1.3 \mathrm{~Hz}, 1 \mathrm{H}), 7.15(\mathrm{t}, J=8.7 \mathrm{~Hz}, 2 \mathrm{H}), 7.04(\mathrm{~d}, J=1.3 \mathrm{~Hz}, 1 \mathrm{H}), 6.87(\mathrm{dd}, J=8.5,1.6 \mathrm{~Hz}, 1 \mathrm{H})$, 6.79 (dd, $J=8.4,1.7 \mathrm{~Hz}, 1 \mathrm{H}), 6.57$ (ddd, $J=5.8,2.1,0.8 \mathrm{~Hz}, 2 \mathrm{H}), 2.53-2.46(\mathrm{~m}, 2 \mathrm{H}), 1.13-0.92(\mathrm{~m}, 4 \mathrm{H})$, $0.64(\mathrm{t}, J=7.0 \mathrm{~Hz}, 3 \mathrm{H}), 0.28(\mathrm{~s}, 6 \mathrm{H})$.

${ }^{13} \mathrm{C} \mathrm{NMR}\left(101 \mathrm{MHz}, \mathrm{CDCl}_{3}\right) \delta 156.5,153.2,152.8,144.7,144.5,140.4,139.3,138.1,137.9,133.9,128.9$, 128.0, 127.9, 126.8, 126.6, 126.2, 125.7, 121.2, 120.9, 110.2, 110.1, 106.7, 106.6, 39.3, 30.8, 22.9, 0.0.

29Si NMR (119 MHz, $\left.\mathrm{CDCl}_{3}\right) \delta-11.5$.

FTIR (cm-1): 2955, 2926, 2857, 1592, 1535, 1465, 1428, 1260, 1200, 1130, 1109, 1031, 832, 770, 737, 701.

HRMS (LIFDI) m/z, calcd for [ $\left.\mathrm{C}_{30} \mathrm{H}_{30} \mathrm{O}_{2} \mathrm{Si}\right]^{+}:$450.2015; found: 450.2021.

\section{Anti-9}

${ }^{1} \mathrm{H} \mathrm{NMR}\left(400 \mathrm{MHz}, \mathrm{CDCl}_{3}\right) \delta 7.62(\mathrm{t}, J=2.2 \mathrm{~Hz}, 2 \mathrm{H}), 7.45(\mathrm{~d}, J=8.4 \mathrm{~Hz}, 1 \mathrm{H}), 7.33(\mathrm{~d}, J=8.4 \mathrm{~Hz}, 1 \mathrm{H}), 7.26$ $-7.13(\mathrm{~m}, 7 \mathrm{H}), 7.03(\mathrm{dd}, J=8.4,1.7 \mathrm{~Hz}, 1 \mathrm{H}), 6.97(\mathrm{dd}, J=8.4,1.7 \mathrm{~Hz}, 1 \mathrm{H}), 6.76(\mathrm{dd}, J=2.1,0.8 \mathrm{~Hz}, 1 \mathrm{H})$, $6.66(\mathrm{dd}, J=2.1,0.8 \mathrm{~Hz}, 1 \mathrm{H}), 2.22-2.14(\mathrm{~m}, 2 \mathrm{H}), 1.13-1.04(\mathrm{~m}, 2 \mathrm{H}), 0.99(\mathrm{dt}, J=14.3,6.9 \mathrm{~Hz}, 2 \mathrm{H}), 0.59$ (t, $J=7.2 \mathrm{~Hz}, 3 \mathrm{H}),-0.19(\mathrm{~s}, 6 \mathrm{H})$.

${ }^{13} \mathrm{C} \mathrm{NMR}\left(101 \mathrm{MHz}, \mathrm{CDCl}_{3}\right) \delta 156.6,154.2,153.4,145.2,145.0,140.4,140.2,139.1,138.5,133.8,128.3$, 127.3, 127.3, 126.8, 125.4, 125.4, 121.8, 120.6, 110.7, 110.5, 106.8, 106.8, 37.3, 30.4, 22.6, 14.0, -0.9.

${ }^{29} \mathrm{Si} \mathrm{NMR}\left(119 \mathrm{MHz}, \mathrm{CDCl}_{3}\right) \delta-12.9$.

FTIR $\left(\mathrm{cm}^{-1}\right): 2955,2928,2858,1591,1537,1461,1428,1261,1228,1130,1109,1032,883,853,833$, $770,735,701$.

HRMS (LIFDI) m/z, calcd for [ $\left.\mathrm{C}_{30} \mathrm{H}_{30} \mathrm{O}_{2} \mathrm{Si}\right]^{+}:$450.2015; found: 450.2025.

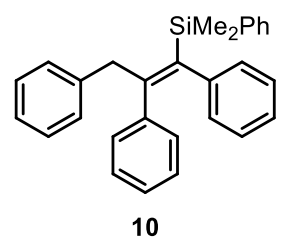

Chemical Formula: $\mathrm{C}_{29} \mathrm{H}_{28} \mathrm{Si}$ Exact Mass: 404.1960 Molecular Weight: 404.6280
(10) According to the general procedure $\mathrm{B},\left(\mathrm{Ph}_{3} \mathrm{P}\right)_{2} \mathrm{PdCl}_{2}(28 \mathrm{mg}, 0.04 \mathrm{mmol}, 2 \mathrm{~mol}$ $\%$ ), diphenyl acetylene (356 mg, $2 \mathrm{mmol}, 1$ equiv), dioxane (1 mL, $2.0[\mathrm{M}]$ ), triethylamine $(836 \mu \mathrm{L}, 6 \mathrm{mmol}, 3$ equiv), and dimethylphenylsilyl iodide $(1.12 \mathrm{~mL}$, $6 \mathrm{mmol}, 3$ equiv) were combined under nitrogen. Benzylzinc iodide $\mathbf{S} 6$ in dioxane ([1.9 M], $1.58 \mathrm{~mL}, 3 \mathrm{mmol}, 1.5$ equiv) was added over $4 \mathrm{~h}$. The reaction was quenched with THF, stirred for $0.25 \mathrm{~h}$, and worked up according to the general procedure. Analysis of the crude reaction mixture via gas chromatography revealed a >95:5 syn:anti ratio. The product was purified by silica gel chromatography ( $1: 99$ ethyl acetate : hexanes) followed by reverse phase column chromatography on a Biotage instrument using a SNAP Ultra C18 $60 \mathrm{~g}$ column (3:17 water : acetonitrile) to afford 10 as a white crystalline solid (634 mg, 78\%).

${ }^{1} \mathrm{H}$ NMR $\left(400 \mathrm{MHz}, \mathrm{CDCl}_{3}\right) \delta 7.70(\mathrm{dd}, J=6.4,3.0 \mathrm{~Hz}, 2 \mathrm{H}), 7.42-7.39(\mathrm{~m}, 3 \mathrm{H}), 7.06(\mathrm{dd}, J=10.3,7.6 \mathrm{~Hz}$, $5 \mathrm{H}), 6.95(\mathrm{~d}, J=7.3 \mathrm{~Hz}, 1 \mathrm{H}), 6.93-6.83(\mathrm{~m}, 7 \mathrm{H}), 6.79-6.74(\mathrm{~m}, 2 \mathrm{H}), 3.84(\mathrm{~s}, 2 \mathrm{H}), 0.34(\mathrm{~s}, 6 \mathrm{H})$.

${ }^{13} \mathrm{C}$ NMR $\left(101 \mathrm{MHz}, \mathrm{CDCl}_{3}\right) \delta 153.3,144.5,142.4,140.9,139.9,138.9,134.0,129.3,129.1,129.1,129.1$, $128.2,128.0,127.5,127.1,125.9,125.8,124.8,44.3,0.1$.

${ }^{29} \mathrm{Si} \mathrm{NMR}\left(119 \mathrm{MHz}, \mathrm{CDCl}_{3}\right) \delta-11.4$.

FTIR $\left(\mathrm{cm}^{-1}\right): 3063,3024,2954,1597,1494,1427,1249,1111,834,810,786,731,698$. 
$\mathrm{mp}=72-73^{\circ} \mathrm{C}$.

HRMS (LIFDI) m/z, calcd for [ $\mathrm{C}_{29} \mathrm{H}_{28} \mathrm{Si}^{+}$: 404.1960 ; found: 404.1957.

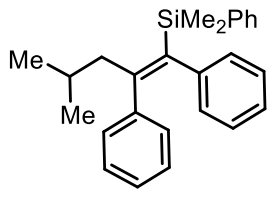

11

Chemical Formula: $\mathrm{C}_{26} \mathrm{H}_{30} \mathrm{Si}$ Exact Mass: 370.2117 Molecular Weight: 370.6110

(11) According to the general procedure $\mathrm{B},\left(\mathrm{Ph}_{3} \mathrm{P}\right)_{2} \mathrm{PdCl}_{2}(28 \mathrm{mg}, 0.04 \mathrm{mmol}, 2 \mathrm{~mol}$ $\%$ ), diphenylacetylene (356 mg, $2.0 \mathrm{mmol}, 1$ equiv), dioxane (1 mL, 2.0 [M]), triethylamine $(836 \mu \mathrm{L}, 6.0 \mathrm{mmol}, 3$ equiv), and dimethylphenylsilyl iodide $(1.12 \mathrm{~mL}$, $6.0 \mathrm{mmol}, 3$ equiv) were combined under nitrogen. Isobutylzinc iodide $\mathbf{S} 4$ in dioxane ([1.0 M], $2.9 \mathrm{~mL}, 3.0 \mathrm{mmol}, 1.5$ equiv) was added over $4 \mathrm{~h}$. The reaction was quenched with water, stirred for $0.25 \mathrm{~h}$, and worked up according to the general procedure. Analysis of the crude reaction mixture via gas chromatography revealed a 92:8 syn:anti ratio. The product was purified by silica gel chromatography ( $1: 99$ to $3: 47$ ethyl acetate : hexanes) followed by reverse phase column chromatography on a Biotage instrument using a SNAP Ultra C18 $60 \mathrm{~g}$ column (7:13 to $0: 100$ water : acetonitrile) to afford $\mathbf{1 1}$ as a white solid (687 $\mathrm{mg}, 93 \%)$.

${ }^{1} \mathrm{H}$ NMR $\left(400 \mathrm{MHz}, \mathrm{CDCl}_{3}\right) \delta 7.74-7.61(\mathrm{~m}, 2 \mathrm{H}), 7.41$ (dd, $\left.J=4.9,1.8 \mathrm{~Hz}, 3 \mathrm{H}\right), 7.11-7.02(\mathrm{~m}, 4 \mathrm{H}), 7.02$ - $6.94(\mathrm{~m}, 2 \mathrm{H}), 6.92(\mathrm{dd}, J=8.2,1.4 \mathrm{~Hz}, 2 \mathrm{H}), 6.82(\mathrm{dd}, J=8.1,1.2 \mathrm{~Hz}, 2 \mathrm{H}), 2.40(\mathrm{~d}, J=7.3 \mathrm{~Hz}, 2 \mathrm{H}), 1.33$ $(\mathrm{p}, J=7.1 \mathrm{~Hz}, 1 \mathrm{H}), 0.64(\mathrm{~d}, J=6.6 \mathrm{~Hz}, 6 \mathrm{H}), 0.30(\mathrm{~s}, 6 \mathrm{H})$.

${ }^{13} \mathrm{C}$ NMR $\left(101 \mathrm{MHz}, \mathrm{CDCl}_{3}\right) \delta 154.8,144.7,142.8,140.3,139.8,134.1,129.5,128.9,128.7,127.9,127.4$, 127.3, 125.7, 124.6, 46.6, 26.4, 22.1, 0.1.

$\left.{ }^{29} \mathrm{Si} \mathrm{NMR} \mathrm{(119} \mathrm{MHz,} \mathrm{CDCl}_{3}\right) \delta-11.9$.

FTIR (cm-1): 2955, 1586, 1487, 1427, 1248, 1112, 833, 810, 699, 471.

$\mathrm{mp}=71^{\circ} \mathrm{C}$.

HRMS (LIFDI) m/z, calcd for [ $\mathrm{C}_{26} \mathrm{H}_{30} \mathrm{Si}^{+}$: 370.2117 ; found: 370.2119 .

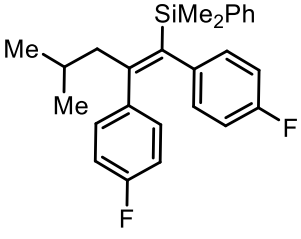

12

Chemical Formula: $\mathrm{C}_{26} \mathrm{H}_{28} \mathrm{~F}_{2} \mathrm{Si}$ Exact Mass: 406.1928

Molecular Weight: 406.5918
(12) According to the general procedure $\mathrm{B},\left(\mathrm{Ph}_{3} \mathrm{P}\right)_{2} \mathrm{PdCl}_{2}(28 \mathrm{mg}, 0.04 \mathrm{mmol}, 2$ mol \%), 1,2-bis(4-fluorophenyl)acetylene (428 mg, $2.0 \mathrm{mmol}, 1$ equiv), dioxane (1 mL, $2.0[\mathrm{M}]$ ), triethylamine ( $836 \mu \mathrm{L}, 6.0 \mathrm{mmol}, 3$ equiv), and dimethylphenylsilyl iodide ( $1.12 \mathrm{~mL}, 6.0 \mathrm{mmol}, 3$ equiv) were combined under nitrogen. Isobutylzinc iodide $\mathbf{S} 4$ in dioxane ( $[0.7 \mathrm{M}], 4.6 \mathrm{~mL}, 3.0 \mathrm{mmol}, 1.5$ equiv) was added over $4 \mathrm{~h}$. The reaction was quenched with water, stirred for $0.25 \mathrm{~h}$, and worked up according to the general procedure. Analysis of the crude reaction mixture via gas chromatography revealed a $>95: 5$ syn:anti ratio. The product was purified by silica gel chromatography $(1: 99$ to $3: 47$ ethyl acetate : hexanes) followed by reverse phase column chromatography on a Biotage instrument using a SNAP

Ultra C18 $60 \mathrm{~g}$ column (7:13 to $0: 100$ water : acetonitrile) to afford 12 as a white solid (667 mg, 82\%).

${ }^{1} \mathrm{H} \mathrm{NMR}\left(400 \mathrm{MHz}, \mathrm{CDCl}_{3}\right) \delta 7.61(\mathrm{dd}, J=6.5,2.9 \mathrm{~Hz}, 2 \mathrm{H}), 7.46-7.33(\mathrm{~m}, 3 \mathrm{H}), 6.83(\mathrm{dd}, J=8.6,5.6 \mathrm{~Hz}$, $2 \mathrm{H}), 6.78-6.68(\mathrm{~m}, 6 \mathrm{H}), 2.36(\mathrm{~d}, J=7.3 \mathrm{~Hz}, 2 \mathrm{H}), 1.31$ (hept, $J=7.0 \mathrm{~Hz}, 1 \mathrm{H}), 0.62(\mathrm{~d}, J=6.6 \mathrm{~Hz}, 6 \mathrm{H}), 0.29$ $(\mathrm{s}, 6 \mathrm{H})$.

${ }^{13} \mathrm{C}$ NMR (101 MHz, CDCl $) \delta 161.9(\mathrm{~d}, J=44.9 \mathrm{~Hz}), 159.5(\mathrm{~d}, J=43.2 \mathrm{~Hz}), 154.5,140.4(\mathrm{~d}, J=3.4 \mathrm{~Hz})$, 139.8, 139.5, 138.5 (d, $J=3.4 \mathrm{~Hz}), 134.0,130.7$ (d, $J=7.7 \mathrm{~Hz}), 130.1$ (d, $J=7.8 \mathrm{~Hz}), 129.1,128.0,114.5$ (d, $J=7.0 \mathrm{~Hz}), 114.3(\mathrm{~d}, J=7.0 \mathrm{~Hz}), 46.5,26.4,22.0,0.1$.

$\left.{ }^{29} \mathrm{Si} \mathrm{NMR} \mathrm{(119} \mathrm{MHz,} \mathrm{CDCl}_{3}\right) \delta-11.8$.

${ }^{19} \mathrm{~F} \mathrm{NMR}\left(565 \mathrm{MHz}, \mathrm{CDCl}_{3}\right) \delta-116.6,-118.6$. 
FTIR (cm-1): 3068, 2957, 2869, 1602, 1503, 1249, 1221, 1157, 835, 816, 798, 700, 531.

$\mathrm{mp}=73-74^{\circ} \mathrm{C}$.

HRMS (LIFDI) m/z, calcd for [ $\left.\mathrm{C}_{26} \mathrm{H}_{28} \mathrm{~F}_{2} \mathrm{Si}\right]^{+}:$406.1928; found: 406.1945.

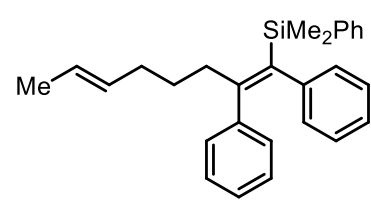

13

Chemical Formula: $\mathrm{C}_{28} \mathrm{H}_{32} \mathrm{Si}$ Exact Mass: 396.2273

Molecular Weight: 396.6490

(13) According to the general procedure $\mathrm{B},\left(\mathrm{Ph}_{3} \mathrm{P}\right)_{2} \mathrm{PdCl}_{2}(28 \mathrm{mg}, 0.04 \mathrm{mmol}, 2$ $\mathrm{mol} \%$ ), diphenyl acetylene (356 mg, $2 \mathrm{mmol}, 1$ equiv), dioxane (1 mL, 2.0 [M]), triethylamine $(836 \mu \mathrm{L}, 6 \mathrm{mmol}, 3$ equiv), and dimethylphenylsilyl iodide $(1.12 \mathrm{~mL}$, $6 \mathrm{mmol}, 3$ equiv) were combined under nitrogen. $(E)$-2-Hexenezinc iodide S5 in dioxane ( $[0.93 \mathrm{M}], 3.3 \mathrm{~mL}, 3 \mathrm{mmol}, 1.5$ equiv) was added over $4 \mathrm{~h}$. The reaction was quenched with THF, stirred for $0.25 \mathrm{~h}$, and worked up according to the general procedure. Analysis of the crude reaction mixture via gas chromatography revealed a >95:5 syn:anti ratio. The product was purified by silica gel chromatography ( $1: 99$ to $5: 95$ ethyl acetate : hexanes) followed by reverse phase column chromatography on a Biotage instrument using a SNAP Ultra C18 $60 \mathrm{~g}$ column ( 3 : 17 water : acetonitrile) to afford 13 as a clear pale yellow oil ( $453 \mathrm{mg}, 57 \%$ ).

${ }^{1} \mathrm{H}$ NMR (400 MHz, CDCl $) \delta 7.75-7.69(\mathrm{~m}, 2 \mathrm{H}), 7.43(\mathrm{dd}, J=5.0,1.9 \mathrm{~Hz}, 3 \mathrm{H}), 7.10-7.04(\mathrm{~m}, 4 \mathrm{H}), 7.00$ $(\mathrm{t}, J=7.3 \mathrm{~Hz}, 1 \mathrm{H}), 6.98-6.91(\mathrm{~m}, 3 \mathrm{H}), 6.87-6.83(\mathrm{~m}, 2 \mathrm{H}), 5.31-5.19(\mathrm{~m}, 1 \mathrm{H}), 5.19-5.10(\mathrm{~m}, 1 \mathrm{H}), 2.57$ $-2.25(\mathrm{~m}, 2 \mathrm{H}), 1.67(\mathrm{q}, J=7.3 \mathrm{~Hz}, 2 \mathrm{H}), 1.58(\mathrm{~d}, J=7.2 \mathrm{~Hz}, 3 \mathrm{H}), 1.24-1.06(\mathrm{~m}, 2 \mathrm{H}), 0.31(\mathrm{~s}, 6 \mathrm{H})$.

${ }^{13} \mathrm{C}$ NMR $\left(101 \mathrm{MHz}, \mathrm{CDCl}_{3}\right) \delta 155.9,144.5,143.0,140.3,138.4,133.9,131.1,129.3,128.9,128.8,128.0$, $127.4,127.3,125.8,125.0,124.6,38.4,32.7,28.5,18.0,-0.1$.

${ }^{29} \mathrm{Si} \mathrm{NMR}\left(119 \mathrm{MHz}, \mathrm{CDCl}_{3}\right) \delta-11.6$.

FTIR $\left(\mathrm{cm}^{-1}\right): 3067,3019,2954,2856,1586,1486,1440,1427,1249,1112,966,834,811,730,699$.

HRMS (LIFDI) m/z, calcd for [ $\left.\mathrm{C}_{28} \mathrm{H}_{32} \mathrm{Si}\right]^{+}:$396.2273; found: 396.2256.

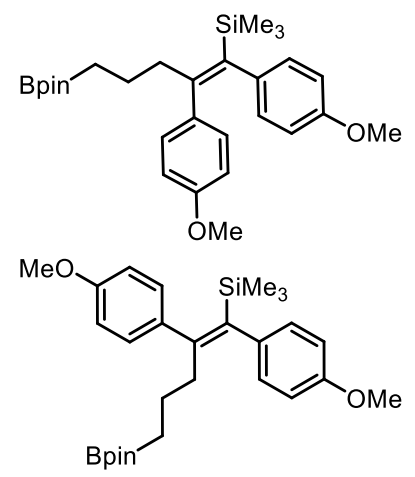

15

Chemical Formula: $\mathrm{C}_{28} \mathrm{H}_{41} \mathrm{O}_{2} \mathrm{Si}$ Exact Mass: 353.1937

Molecular Weight: 353.5570
(15) According to the general procedure $\mathrm{B},\left(\mathrm{Ph}_{3} \mathrm{P}\right)_{2} \mathrm{PdCl}_{2}(28 \mathrm{mg}, 0.04 \mathrm{mmol}, 2$ mol \%), 1,2-bis(4-methoxyphenyl)acetylene (476 mg, $2 \mathrm{mmol}, 1$ equiv), dioxane (1 $\mathrm{mL}, 2.0[\mathrm{M}])$, triethylamine (836 $\mu \mathrm{L}, 6 \mathrm{mmol}, 3$ equiv), and trimethylsilyl iodide ( $853 \mu \mathrm{L}, 6 \mathrm{mmol}, 3$ equiv) were combined under nitrogen. 3-(4,4,5,5,-tetramethyl-1,3,2-dioxaborolan-2-yl)zinc iodide $\mathbf{S 7}$ in dioxane ([0.55 M], $5.46 \mathrm{~mL}, 3 \mathrm{mmol}, 1.5$ equiv) was added over $4 \mathrm{~h}$. The reaction was quenched with THF, stirred for $0.25 \mathrm{~h}$, and worked up according to the general procedure. Analysis of the crude reaction mixture via gas chromatography revealed a 50:50 syn:anti ratio. The product was purified by silica gel chromatography (1:99 ethyl acetate : hexanes) followed by reverse phase column chromatography on a Biotage instrument using a SNAP Ultra C18 60 g column ( $3: 17$ water : acetonitrile) to afford 15 as a clear pale yellow oil (688 $\mathrm{mg}, 97 \%$, mixture of syn and anti isomers).

${ }^{1} \mathrm{H}$ NMR $\left(400 \mathrm{MHz}, \mathrm{CDCl}_{3}\right) \delta 7.12(\mathrm{~d}, J=8.7 \mathrm{~Hz}, 2 \mathrm{H}), 6.93-6.78(\mathrm{~m}, 8 \mathrm{H}), 6.64-6.53(\mathrm{~m}, 6 \mathrm{H}), 3.83(\mathrm{~s}$, $3 \mathrm{H}), 3.81(\mathrm{~s}, 3 \mathrm{H}), 3.69(\mathrm{~s}, 6 \mathrm{H}), 2.65-2.49(\mathrm{~m}, 2 \mathrm{H}), 2.19-2.08(\mathrm{~m}, 2 \mathrm{H}), 1.39(\mathrm{p}, J=8.0 \mathrm{~Hz}, 2 \mathrm{H}), 1.21(\mathrm{~s}$, $14 \mathrm{H}), 1.11(\mathrm{~s}, 12 \mathrm{H}), 0.76(\mathrm{t}, J=7.8 \mathrm{~Hz}, 2 \mathrm{H}), 0.52(\mathrm{t}, J=7.8 \mathrm{~Hz}, 2 \mathrm{H}), 0.11(\mathrm{~s}, 9 \mathrm{H}),-0.36(\mathrm{~s}, 9 \mathrm{H})$. 
${ }^{13} \mathrm{C} \mathrm{NMR}\left(101 \mathrm{MHz}, \mathrm{CDCl}_{3}\right) \delta 158.6,157.2,157.2,156.5,154.0,153.8,141.2,139.6,137.6,137.1,136.9$, $135.8,130.1,130.0,130.0,129.3,113.4,113.1,112.7,112.6,83.0,82.9,55.4,55.2,55.1,40.9,39.5,25.0$, 24.8, 23.3, 22.3, 1.2, 0.5 .

${ }^{11} \mathrm{~B}$ NMR $\left(193 \mathrm{MHz}, \mathrm{CDCl}_{3}\right) \delta-34.1$.

${ }^{29} \mathrm{Si} \mathrm{NMR}\left(119 \mathrm{MHz}, \mathrm{CDCl}_{3}\right) \delta-7.8,-7.6$.

FTIR $\left(\mathrm{cm}^{-1}\right):$ 2976, 2951, 1608, 1508, 1372, 1243, 1173, 1145, 1036, 838.

$\mathrm{mp}=72-73^{\circ} \mathrm{C}$.

HRMS (FD) m/z, calcd for [ $\mathrm{C}_{28} \mathrm{H}_{41} \mathrm{BO}_{4} \mathrm{Si}^{+}:$: 480.2867; found: 480.2879 .

FTIR $\left(\mathrm{cm}^{-1}\right):$ 3067, 3019, 2956, 2928, 2859, 1486, 1427, 1248, 1112, 834, 810, 730, 699.

HRMS (LIFDI) m/z, calcd for [ $\left.\mathrm{C}_{26} \mathrm{H}_{28} \mathrm{SiCl}_{2}\right]^{+}: 438.1337$; found: 438.1315.

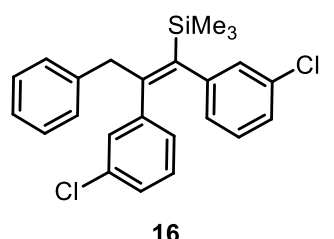

Chemical Formula: $\mathrm{C}_{24} \mathrm{H}_{24} \mathrm{Cl}_{2} \mathrm{Si}$

Exact Mass: 410.1024

Molecular Weight: 411.4410

(16) According to the general procedure $\mathrm{B},\left(\mathrm{Ph}_{3} \mathrm{P}\right)_{2} \mathrm{PdCl}_{2}(28 \mathrm{mg}, 0.04 \mathrm{mmol}, 2$ mol \%), 1,2-bis(3-chlorophenyl)acetylene (494 mg, $2.0 \mathrm{mmol}, 1$ equiv), dioxane (1 mL, $2.0[\mathrm{M}])$, triethylamine $(836 \mu \mathrm{L}, 6.0 \mathrm{mmol}, 3$ equiv), and trimethylsilyl iodide ( $853 \mu \mathrm{L}, 6.0 \mathrm{mmol}, 3$ equiv) were combined under nitrogen. Benzylzinc iodide $\mathbf{S} 6$ in dioxane ([0.8 M], $3.8 \mathrm{~mL}, 3.0 \mathrm{mmol}, 1.5$ equiv) was added over $4 \mathrm{~h}$. The reaction was quenched with water, stirred for $0.25 \mathrm{~h}$, and worked up according to the general procedure. Analysis of the crude reaction mixture via gas chromatography revealed a 94:6 syn:anti ratio. The product was purified by silica gel chromatography $(1: 99$ to $3: 47$ ethyl acetate : hexanes) followed by reverse phase column chromatography on a Biotage instrument using a SNAP Ultra C18 $60 \mathrm{~g}$ column (7: 13 to $1: 9$ water : acetonitrile) to afford 16 as a white solid (622 $\mathrm{mg}, 76 \%)$.

${ }^{1} \mathrm{H}$ NMR $\left(600 \mathrm{MHz}, \mathrm{CDCl}_{3}\right) \delta 7.21(\mathrm{t}, J=7.5 \mathrm{~Hz}, 2 \mathrm{H}), 7.13(\mathrm{dd}, J=16.0,7.3 \mathrm{~Hz}, 3 \mathrm{H}), 7.00(\mathrm{t}, J=7.8 \mathrm{~Hz}$, $1 \mathrm{H}), 6.95(\mathrm{dt}, J=8.0,1.5 \mathrm{~Hz}, 1 \mathrm{H}), 6.89-6.74(\mathrm{~m}, 4 \mathrm{H}), 6.67(\mathrm{dd}, J=7.5,1.2 \mathrm{~Hz}, 1 \mathrm{H}), 6.60-6.54(\mathrm{~m}, 1 \mathrm{H})$, $3.98(\mathrm{~s}, 5 \mathrm{H}), 0.20(\mathrm{~s}, 9 \mathrm{H})$.

${ }^{13} \mathrm{C}$ NMR $\left(151 \mathrm{MHz}, \mathrm{CDCl}_{3}\right) \delta 151.0,146.2,144.1,143.0,138.3,133.5,133.2,129.0,128.9,128.8,128.7$, $128.5,128.4,127.4,127.1,126.4,126.2,125.2,43.7,1.1$.

${ }^{29} \mathrm{Si} \mathrm{NMR}\left(119 \mathrm{MHz}, \mathrm{CDCl}_{3}\right) \delta-6.5$.

FTIR $\left(\mathrm{cm}^{-1}\right)$ : 2953, 1589, 1560, 1251, 933, 839, 789, 762, 696.

$\mathrm{mp}=82-83^{\circ} \mathrm{C}$.

HRMS (LIFDI) m/z, calcd for [ $\left.\mathrm{C}_{24} \mathrm{H}_{24} \mathrm{Cl}_{2} \mathrm{Si}\right]^{+}: 410.1024$; found: 410.1024 .

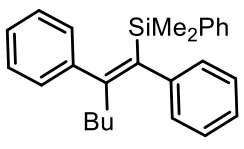

28

Chemical Formula: $\mathrm{C}_{26} \mathrm{H}_{30} \mathrm{Si}$ Exact Mass: 370.2117

Molecular Weight: 370.6110

(28) According to the general procedure $\mathrm{B}$, (JessePhos) ${ }_{2} \mathrm{PdCl}_{2}$ (44 mg, $0.04 \mathrm{mmol}$, $2 \mathrm{~mol} \%$ ), diphenylacetylene (357 mg, $2.0 \mathrm{mmol}, 1$ equiv), dioxane (1 mL, $2.0[\mathrm{M}])$, triethylamine $(836 \mu \mathrm{L}, 6.0 \mathrm{mmol}, 3$ equiv), and dimethylphenylsilyl iodide $(1.12 \mathrm{~mL}$, $6.0 \mathrm{mmol}, 3$ equiv) were combined under nitrogen. $n$-Butylzinc iodide $\mathbf{S} 3$ in dioxane ([2.0 M], $1.6 \mathrm{~mL}, 3.0 \mathrm{mmol}, 1.5$ equiv) was added over $4 \mathrm{~h}$. The reaction was quenched with water, stirred for $0.25 \mathrm{~h}$, and worked up according to the general procedure. Analysis of the crude reaction mixture via gas chromatography revealed a 13:87 syn:anti ratio. The product was purified by silica gel chromatography (1:99 to $3: 47$ ethyl acetate : hexanes) followed by reverse phase column 
chromatography on a Biotage instrument using a SNAP Ultra C18 $60 \mathrm{~g}$ column (7:13 to $0: 100$ water : acetonitrile) to afford 28 as a clear colorless oil (565 $\mathrm{mg}, 76 \%$ ).

${ }^{1} \mathrm{H} \mathrm{NMR}\left(600 \mathrm{MHz}, \mathrm{CDCl}_{3}\right) \delta 7.31(\mathrm{q}, J=6.0,4.7 \mathrm{~Hz}, 5 \mathrm{H}), 7.28-7.23(\mathrm{~m}, 5 \mathrm{H}), 7.20(\mathrm{t}, J=7.4 \mathrm{~Hz}, 1 \mathrm{H}), 7.16$ $-7.09(\mathrm{~m}, 2 \mathrm{H}), 7.04-7.00(\mathrm{~m}, 2 \mathrm{H}), 2.26-2.11(\mathrm{~m}, 2 \mathrm{H}), 1.10$ (ddt, $J=35.4,14.7,7.1 \mathrm{~Hz}, 4 \mathrm{H}), 0.67(\mathrm{t}, J=$ $7.2 \mathrm{~Hz}, 3 \mathrm{H}),-0.12(\mathrm{~s}, 6 \mathrm{H})$.

${ }^{13} \mathrm{C} \mathrm{NMR}\left(151 \mathrm{MHz}, \mathrm{CDCl}_{3}\right) \delta 156.0,144.4,143.9,140.3,139.9,134.0,129.1,128.8,128.4,127.9,127.8$, $127.4,126.9,125.3,37.1,30.3,22.6,13.9,-1.0$.

29Si NMR (119 MHz, $\left.\mathrm{CDCl}_{3}\right) \delta-12.6$.

FTIR $\left(\mathrm{cm}^{-1}\right): 3068,3022,2956,2927,2858,1596,1442,1427,1247,1110,833,812,770,701,471$.

HRMS (LIFDI) m/z, calcd for [ $\left.\mathrm{C}_{26} \mathrm{H}_{30} \mathrm{Si}\right]^{+}: 370.2117$; found: 370.2173 .

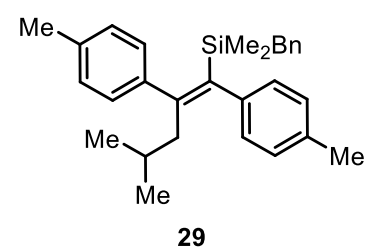

Chemical Formula: $\mathrm{C}_{29} \mathrm{H}_{36} \mathrm{Si}$ Exact Mass: 412.2586 Molecular Weight: 412.6920

(29) According to the general procedure $\mathrm{B}$, (JessePhos) ${ }_{2} \mathrm{PdCl}_{2}$ (44 mg, 0.04 mmol, $2 \mathrm{~mol} \%$ ), 1,2-bis(4-methylphenyl)acetylene (413 mg, $2.0 \mathrm{mmol}, 1$ equiv), dioxane $(1 \mathrm{~mL}, 2.0[\mathrm{M}])$, triethylamine $(0.836 \mathrm{~mL}, 6.0 \mathrm{mmol}, 3$ equiv), and dimethylbenzylsilyl iodide ( $1.13 \mathrm{~mL}, 6.0 \mathrm{mmol}, 3$ equiv) were combined under nitrogen. Isobutylzinc iodide $\mathbf{S} 4$ in dioxane ([1.3 M], $2.5 \mathrm{~mL}, 3.0 \mathrm{mmol}, 1.5$ equiv) was added over $4 \mathrm{~h}$. The reaction was quenched with water, stirred for $0.25 \mathrm{~h}$, and worked up according to the general procedure. Analysis of the crude reaction mixture via gas chromatography revealed $a<5: 95$ syn:anti ratio. The product was purified by silica gel chromatography (1:99 to $3: 47$ ethyl acetate : hexanes)

followed by reverse phase column chromatography on a Biotage instrument using a SNAP Ultra C18 $60 \mathrm{~g}$ column ( $7: 13$ to $0: 100$ water : acetonitrile) to afford 29 as a white solid ( $208 \mathrm{mg}, 50 \%)$.

${ }^{1} \mathrm{H}$ NMR $\left(600 \mathrm{MHz}, \mathrm{CDCl}_{3}\right) \delta 7.2-7.1(\mathrm{~m}, 6 \mathrm{H}), 7.1(\mathrm{~d}, J=8.0 \mathrm{~Hz}, 2 \mathrm{H}), 7.0-7.0(\mathrm{~m}, 1 \mathrm{H}), 6.9-6.8(\mathrm{~m}$, 4H), $2.4(\mathrm{~d}, J=5.6 \mathrm{~Hz}, 6 \mathrm{H}), 2.1(\mathrm{~d}, J=7.2 \mathrm{~Hz}, 2 \mathrm{H}), 1.8(\mathrm{~s}, 2 \mathrm{H}), 1.3$ (hept, $J=6.8 \mathrm{~Hz}, 1 \mathrm{H}), 0.7(\mathrm{~d}, J=6.6$ $\mathrm{Hz}, 6 \mathrm{H}),-0.5(\mathrm{~s}, 6 \mathrm{H})$.

${ }^{13} \mathrm{C}$ NMR $\left(101 \mathrm{MHz}, \mathrm{CDCl}_{3}\right) \delta 154.4,141.5,141.3,140.8,140.7,136.6,134.5,128.8,128.7,128.6,128.6$, $128.5,128.1,123.9,45.9,26.4,26.0,22.5,21.4,21.3,-1.6$.

${ }^{29} \mathrm{Si} \mathrm{NMR}\left(119 \mathrm{MHz}, \mathrm{CDCl}_{3}\right) \delta-7.4$.

FTIR (cm-1): 3024, 2955, 2928, 2869, 1600, 1493, 1452, 1248, 1205, 1154, 833, 761, 698.

$\mathrm{mp}=89-90^{\circ} \mathrm{C}$

HRMS (LIFDI) m/z, calcd for [ $\left.\mathrm{C}_{24} \mathrm{H}_{24} \mathrm{~F}_{2} \mathrm{Sii}\right]^{+}: 412.2586$; found: 412.2586 .

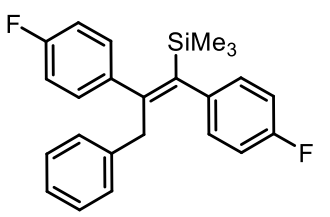

30

Chemical Formula: $\mathrm{C}_{24} \mathrm{H}_{24} \mathrm{~F}_{2} \mathrm{Si}$ Exact Mass: 378.1615

Molecular Weight: 378.5378
(30) According to the general procedure $\mathrm{B}$, (JessePhos) ${ }_{2} \mathrm{PdCl}_{2}$ (44 mg, 0.04 mmol, $2 \mathrm{~mol} \%$ ), 1,2-bis(4-fluorophenyl)acetylene (428 mg, $2.0 \mathrm{mmol}$, 1 equiv), dioxane (1 mL, $2.0[\mathrm{M}])$, triethylamine $(836 \mu \mathrm{L}, 6.0 \mathrm{mmol}, 3$ equiv), and trimethylsilyl iodide ( $853 \mu \mathrm{L}, 6.0 \mathrm{mmol}, 3$ equiv) were combined under nitrogen. Benzylzinc iodide $\mathbf{S} 6$ in dioxane ([0.8 M], $3.8 \mathrm{~mL}, 3.0 \mathrm{mmol}, 1.5$ equiv) was added over $4 \mathrm{~h}$. The reaction was quenched with water, stirred for $0.25 \mathrm{~h}$, and worked up according to the general procedure. Analysis of the crude reaction mixture via gas chromatography revealed a $<5: 95$ syn:anti ratio. The product was purified by silica gel chromatography ( $1: 99$ to $3: 47$ ethyl acetate : hexanes) followed by reverse phase column chromatography on a Biotage instrument using a SNAP Ultra C18 $60 \mathrm{~g}$ column (7 : 13 to $0: 100$ water : acetonitrile) to afford 30 as a white solid (544 mg, 72\%). 
${ }^{1} \mathrm{H}$ NMR $\left(600 \mathrm{MHz}, \mathrm{CDCl}_{3}\right) \delta 7.14-7.02(\mathrm{~m}, 7 \mathrm{H}), 6.96(\mathrm{dd}, J=8.7,5.6 \mathrm{~Hz}, 2 \mathrm{H}), 6.90(\mathrm{t}, J=8.7 \mathrm{~Hz}, 2 \mathrm{H})$, $6.80-6.71(\mathrm{~m}, 2 \mathrm{H}), 3.48(\mathrm{~s}, 3 \mathrm{H}),-0.32(\mathrm{~s}, 9 \mathrm{H})$.

${ }^{13} \mathrm{C}$ NMR $\left(101 \mathrm{MHz}, \mathrm{CDCl}_{3}\right) \delta 162.8(\mathrm{~d}, J=84.9 \mathrm{~Hz}), 160.4(\mathrm{~d}, J=83.2 \mathrm{~Hz}), 151.7,142.5,140.1(\mathrm{~d}, J=3.4$ $\mathrm{Hz}), 139.1$ (d, $J=3.3 \mathrm{~Hz}), 138.9,130.8(\mathrm{~d}, J=7.9 \mathrm{~Hz}), 129.7(\mathrm{~d}, J=7.7 \mathrm{~Hz}), 129.2,128.1,125.9,115.2$ (d, $J=21.1 \mathrm{~Hz}), 114.6(\mathrm{~d}, J=21.1 \mathrm{~Hz}), 43.4,0.3$.

${ }^{29} \mathrm{Si} \mathrm{NMR}\left(119 \mathrm{MHz}, \mathrm{CDCl}_{3}\right) \delta-6.7$.

${ }^{19} \mathrm{~F} \mathrm{NMR}\left(565 \mathrm{MHz}, \mathrm{CDCl}_{3}\right) \delta-115.6,-117.9$.

FTIR (cm-1): 3029, 2956, 2896, 1601, 1506, 1246, 1223, 1155, 838, 826, 762, 700.

$\mathrm{mp}=93-94^{\circ} \mathrm{C}$.

HRMS (LIFDI) m/z, calcd for [ $\left.\mathrm{C}_{24} \mathrm{H}_{24} \mathrm{~F}_{2} \mathrm{Si}\right]^{+}: 378.1615$; found: 378.1612 .

\section{General Procedure C: Alkyl Alkynes}

Note: THF was used to quench certain reactions to avoid disiloxane formation upon aqueous workup. This quench generates the more easily separated (4-iodobutoxy)silane through silyl-iodide induced ring opening of THF.

A $25 \mathrm{~mL}$ Schlenk flask equipped with a magnetic stirbar and rubber septum was flame-dried and allowed to cool to room temperature under vacuum and refilled with nitrogen. The flask was briefly opened, charged with palladium precatalyst ( 0.05 equiv), and the septum was replaced. The flask was evacuated and refilled with $\mathrm{N}_{2}$ three times. Dioxane $(0.5 \mathrm{~mL})$, triethylamine (5 equiv), silyl-iodide (5 equiv), and alkyne (1 equiv) were added sequentially via syringe at room temperature with stirring. A solution of alkylzinc iodide in dioxane (5 equiv) was then added dropwise over $4 \mathrm{~h}$ via syringe pump. The reaction was allowed to stir at room temperature for $0.25 \mathrm{~h}$ after the addition was completed. The reaction was quenched as indicated and opened to air. The resultant mixture was transferred to a separatory funnel and partitioned between 1 $\mathrm{N}$ aqueous hydrogen chloride solution $(3 \mathrm{~mL})$ to solubilize the zinc salts, water $(10 \mathrm{~mL})$, and diethyl ether $(20 \mathrm{~mL})$. The aqueous layer was extracted with diethyl ether $(3 \times 15 \mathrm{~mL})$. The combined organic layers were washed with saturated aqueous sodium chloride solution $(30 \mathrm{~mL})$ and dried over anhydrous magnesium sulfate, filtered, and concentrated in vacuo. A small aliquot of the crude reaction mixture ( 20 $\mu \mathrm{L}$ ) was analyzed by gas chromatography or NMR spectroscopy to determine the syn:anti ratio. The crude material was purified via silica column chromatography in the indicated solvent combination followed by reverse phase column chromatography on a Biotage instrument using a SNAP Ultra C18 $60 \mathrm{~g}$ column in the indicated

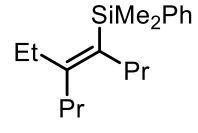

16

Chemical Formula: $\mathrm{C}_{18} \mathrm{H}_{30} \mathrm{Si}$ Exact Mass: 274.2117 Molecular Weight: 274.5230
(16) According to the general procedure C, (DrewPhos) ${ }_{2} \mathrm{Pdl}_{2}(156 \mathrm{mg}, 0.1 \mathrm{mmol}$, $5 \mathrm{~mol} \%)$, dioxane $(1 \mathrm{~mL})$, triethylamine $(1.4 \mathrm{~mL}, 10 \mathrm{mmol}, 5$ equiv), dimethylphenylsilyl iodide (1.80 mL, $10 \mathrm{mmol}, 5$ equiv), and 4-octyne $(293 \mu \mathrm{L}, 2$ $\mathrm{mmol}, 1$ equiv) were combined under nitrogen. Ethylzinc iodide $\mathbf{S} 2$ in dioxane ([1.6 $\mathrm{M}$ ], $6.25 \mathrm{~mL}, 10 \mathrm{mmol}, 5$ equiv) was added over $4 \mathrm{~h}$. The reaction was worked up according to the general procedure. Analysis of the crude reaction mixture via gas chromatography revealed a 92:8 syn:anti ratio. The product was purified by silica gel chromatography (hexanes) followed by reverse phase column chromatography on a Biotage instrument using a SNAP Ultra C18 $60 \mathrm{~g}$ column (3:17 water : acetonitrile) to afford 16 as a clear colorless oil (502 mg, 91\%).

${ }^{1} \mathrm{H}$ NMR $\left(400 \mathrm{MHz}, \mathrm{CDCl}_{3}\right) \delta 7.58-7.42(\mathrm{~m}, 2 \mathrm{H}), 7.36-7.27(\mathrm{~m}, 3 \mathrm{H}), 2.11(\mathrm{td}, J=8.2,2.9 \mathrm{~Hz}, 4 \mathrm{H}), 1.99$ (q, $J=7.4 \mathrm{~Hz}, 2 \mathrm{H}), 1.42(\mathrm{dd}, 2 \mathrm{H}), 1.30(\mathrm{dd}, J=15.9,7.6 \mathrm{~Hz}, 2 \mathrm{H}), 0.94(\mathrm{t}, J=7.3 \mathrm{~Hz}, 3 \mathrm{H}), 0.89$ (t, $J=7.3$ $\mathrm{Hz}, 3 \mathrm{H}), 0.77$ (t, $J=7.4 \mathrm{~Hz}, 3 \mathrm{H}), 0.35(\mathrm{~s}, 6 \mathrm{H})$. 
${ }^{13} \mathrm{C} \mathrm{NMR}\left(101 \mathrm{MHz}, \mathrm{CDCl}_{3}\right) \delta 155.0,141.5,133.9,130.3,128.5,127.7,34.2,32.8,29.9,24.7,22.4,14.7$, $14.5,13.4,0.2$.

$\left.{ }^{29} \mathrm{Si} \mathrm{NMR} \mathrm{(119} \mathrm{MHz,} \mathrm{CDCl}_{3}\right) \delta-11.1$.

FTIR (cm-1): 2959, 2930, 2870, 1595, 1464, 1428, 1248, 1107, 819, 770, 700.

HRMS (LIFDI) m/z, calcd for [ $\left.\mathrm{C}_{16} \mathrm{H}_{27} \mathrm{OSi}\right]^{+}: 274.2117$; found: 274.2108.

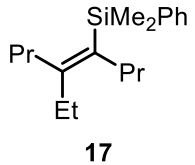

Chemical Formula: $\mathrm{C}_{18} \mathrm{H}_{30} \mathrm{Si}$ Exact Mass: 274.2117 Molecular Weight: 274.5230

(17) According to the general procedure $\mathrm{C}$, (JessePhos) ${ }_{2} \mathrm{PdCl}_{2}(111 \mathrm{mg}, 0.1 \mathrm{mmol}$, $5 \mathrm{~mol} \%)$, dioxane $(1 \mathrm{~mL})$, triethylamine $(1.4 \mathrm{~mL}, 10 \mathrm{mmol}, 5$ equiv), dimethylphenylsilyl iodide (1.80 mL, $10 \mathrm{mmol}, 5$ equiv), and 4-octyne $(293 \mu \mathrm{L}, 2$ $\mathrm{mmol}, 1$ equiv) were combined under nitrogen. Ethylzinc iodide $\mathbf{S} 2$ in dioxane ([1.6 $\mathrm{M}$ ], $6.25 \mathrm{~mL}, 10 \mathrm{mmol}, 5$ equiv) was added over $4 \mathrm{~h}$. The reaction was worked up according to the general procedure. Analysis of the crude reaction mixture via gas chromatography revealed $a<5: 95$ syn:anti ratio. The product was purified by silica gel chromatography (hexanes) followed by reverse phase column chromatography on a Biotage instrument using a SNAP Ultra C18 $60 \mathrm{~g}$ column ( $3: 17$ water : acetonitrile) to afford 17 as a clear colorless oil (420 mg, 77\%).

${ }^{1} \mathrm{H}$ NMR $\left(600 \mathrm{MHz}, \mathrm{CDCl}_{3}\right) \delta 7.55-7.46(\mathrm{~m}, 2 \mathrm{H}), 7.33-7.29(\mathrm{~m}, 3 \mathrm{H}), 2.14(\mathrm{q}, J=7.6 \mathrm{~Hz}, 2 \mathrm{H}), 2.12-2.07$ $(\mathrm{m}, 2 \mathrm{H}), 1.96-1.89(\mathrm{~m}, 2 \mathrm{H}), 1.29(\mathrm{dt}, J=15.0,7.4 \mathrm{~Hz}, 2 \mathrm{H}), 1.19(\mathrm{dt}, 2 \mathrm{H}), 1.00(\mathrm{t}, J=7.5 \mathrm{~Hz}, 3 \mathrm{H}), 0.88$ (t, $J=7.3 \mathrm{~Hz}, 3 \mathrm{H}), 0.64(\mathrm{t}, J=7.3 \mathrm{~Hz}, 3 \mathrm{H}), 0.36(\mathrm{~s}, 6 \mathrm{H})$.

${ }^{13} \mathrm{C}$ NMR $\left(151 \mathrm{MHz}, \mathrm{CDCl}_{3}\right) \delta 155.4,141.5,133.9,130.2,128.5,127.7,38.9,34.2,24.7,24.2,22.3,14.5$, $14.2,13.9,0.1$.

${ }^{29} \mathrm{Si} \mathrm{NMR}\left(119 \mathrm{MHz}, \mathrm{CDCl}_{3}\right) \delta-11.2$.

FTIR $\left(\mathrm{cm}^{-1}\right):$ 2959, 2930, 2871, 1597, 1464, 1427, 1248, 1108, 818, 770, 700.

HRMS (LIFDI) m/z, calcd for [ $\left.\mathrm{C}_{16} \mathrm{H}_{27} \mathrm{OSi}\right]^{+}: 274.2117$; found: 274.2119.

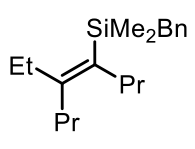

18

Chemical Formula: $\mathrm{C}_{19} \mathrm{H}_{32} \mathrm{Si}$ Exact Mass: 288.2273

Molecular Weight: 288.5500

(18) According to the general procedure $\mathrm{C}$, (DrewPhos) ${ }_{2} \mathrm{Pdl}_{2}$ (156 mg, $0.1 \mathrm{mmol}$, $5 \mathrm{~mol} \%)$, dioxane $(1 \mathrm{~mL})$, triethylamine $(1.4 \mathrm{~mL}, 10 \mathrm{mmol}, 5$ equiv), dimethylbenzylsilyl iodide ( $1.80 \mathrm{~mL}, 10 \mathrm{mmol}, 5$ equiv), and 4-octyne $(293 \mu \mathrm{L}, 2$ $\mathrm{mmol}, 1$ equiv) were combined under nitrogen. Ethylzinc iodide $\mathbf{S 2}$ in dioxane ([1.6 M], $6.25 \mathrm{~mL}, 10 \mathrm{mmol}, 5$ equiv) was added over $4 \mathrm{~h}$. The reaction was worked up according to the general procedure. Analysis of the crude reaction mixture via gas chromatography revealed a >95:5 syn:anti ratio. The product was purified by silica gel chromatography (hexanes) followed by reverse phase column chromatography on a Biotage instrument using a SNAP Ultra C18 $60 \mathrm{~g}$ column (3:17 water : acetonitrile) to afford 18 as a clear colorless oil (496 mg, 86\%).

${ }^{1} \mathrm{H} \mathrm{NMR}\left(600 \mathrm{MHz}, \mathrm{CDCl}_{3}\right) \delta 7.17(\mathrm{t}, J=7.7 \mathrm{~Hz}, 2 \mathrm{H}), 7.04(\mathrm{tt}, J=7.3,1.3 \mathrm{~Hz}, 1 \mathrm{H}), 7.01-6.95(\mathrm{~m}, 2 \mathrm{H}), 2.19$ (s, 2H), 2.11 (q, J = 7.4 Hz, 2H), $2.09-2.02(\mathrm{~m}, 2 \mathrm{H}), 1.97-1.86(\mathrm{~m}, 2 \mathrm{H}), 1.47-1.29(\mathrm{~m}, 2 \mathrm{H}), 1.13$ (ddd, $J=12.4,9.3,5.7 \mathrm{~Hz}, 2 \mathrm{H}), 0.98(\mathrm{t}, J=7.4 \mathrm{~Hz}, 3 \mathrm{H}), 0.93(\mathrm{t}, J=7.3 \mathrm{~Hz}, 3 \mathrm{H}), 0.85(\mathrm{t}, J=7.3 \mathrm{~Hz}, 3 \mathrm{H}), 0.08(\mathrm{~s}$, $6 \mathrm{H})$.

${ }^{13} \mathrm{C}$ NMR $\left(151 \mathrm{MHz}, \mathrm{CDCl}_{3}\right) \delta 154.2,140.8,131.1,128.5,128.1,123.9,34.2,33.0,29.4,27.5,24.4,22.3$, $14.7,14.5,14.1,-0.8$.

${ }^{29} \mathrm{Si} \mathrm{NMR}\left(119 \mathrm{MHz}, \mathrm{CDCl}_{3}\right) \delta-6.8$. 
FTIR (cm-1): 2959, 2930, 2871, 1600, 1493, 1453, 1248, 1206, 1155, 829, 760, 698, 477.

HRMS (LIFDI) m/z, calcd for [ $\left.\mathrm{C}_{16} \mathrm{H}_{27} \mathrm{OSi}\right]^{+}:$288.2273; found: 288.2263 .

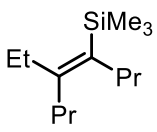

19

Chemical Formula: $\mathrm{C}_{13} \mathrm{H}_{28} \mathrm{Si}$ Exact Mass: 212.1960 Molecular Weight: 212.4520

(19) According to the general procedure C, (DrewPhos) ${ }_{2} \mathrm{Pdl}_{2}(156 \mathrm{mg}, 0.1 \mathrm{mmol}$, $5 \mathrm{~mol} \%)$, dioxane $(1 \mathrm{~mL})$, triethylamine $(1.4 \mathrm{~mL}, 10 \mathrm{mmol}, 5$ equiv), trimethylsilyl iodide ( $1.40 \mathrm{~mL}, 10 \mathrm{mmol}, 5$ equiv), and 4-octyne (293 $\mu \mathrm{L}, 2 \mathrm{mmol}, 1$ equiv) were combined under nitrogen. Ethylzinc iodide $\mathbf{S 2}$ in dioxane ([1.6 M], $6.25 \mathrm{~mL}, 10$ mmol, 5 equiv) was added over $4 \mathrm{~h}$. The reaction was worked up according to the general procedure. Analysis of the crude reaction mixture via gas chromatography revealed a >95:5 syn:anti ratio. The product was purified by silica gel chromatography (hexanes) followed by reverse phase column chromatography on a Biotage instrument using a SNAP Ultra C18 $60 \mathrm{~g}$ column (3:17 water : acetonitrile) to afford 19 as a clear colorless oil (312 $\mathrm{mg}, 73 \%$ ).

${ }^{1} \mathrm{H}$ NMR $\left(600 \mathrm{MHz}, \mathrm{CDCl}_{3}\right) \delta 2.13(\mathrm{q}, J=7.4 \mathrm{~Hz}, 2 \mathrm{H}), 2.08-2.03(\mathrm{~m}, 2 \mathrm{H}), 2.03-1.98(\mathrm{~m}, 2 \mathrm{H}), 1.42-1.32$ (m, 2H), $1.28-1.15(\mathrm{~m}, 2 \mathrm{H}), 0.97(\mathrm{t}, J=7.4 \mathrm{~Hz}, 3 \mathrm{H}), 0.90$ (dt, $J=10.9,7.3 \mathrm{~Hz}, 6 \mathrm{H}), 0.12(\mathrm{~s}, 9 \mathrm{H})$.

${ }^{13} \mathrm{C}$ NMR $\left(151 \mathrm{MHz}, \mathrm{CDCl}_{3}\right) \delta 153.0,132.8,34.1,32.9,29.3,24.6,22.4,14.6,14.5,14.1,1.3$.

$\left.{ }^{29} \mathrm{Si} \mathrm{NMR} \mathrm{(119} \mathrm{MHz,} \mathrm{CDCl}_{3}\right) \delta-7.9$.

FTIR $\left(\mathrm{cm}^{-1}\right): 2959,2931,2871,1465,1248,849,835,757$.

HRMS (ESI) m/z, calcd for [ $\left.\mathrm{C}_{16} \mathrm{H}_{27} \mathrm{OSi}\right]^{+}: 213.2033$; found: 213.1841.

20

Chemical Formula: $\mathrm{C}_{22} \mathrm{H}_{36} \mathrm{~S}$ Exact Mass: 328.2586

Molecular Weight: 328.6150

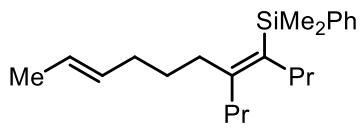

(20) According to the general procedure $\mathrm{C}$, (DrewPhos) ${ }_{2} \mathrm{PdCl}_{2}$ (78 $\mathrm{mg}, 0.05$ $\mathrm{mmol}, 5 \mathrm{~mol} \%)$, triethylamine (700 $\mu \mathrm{L}, 5 \mathrm{mmol}, 5$ equiv), dimethylphenylsilyl iodide (940 $\mu \mathrm{L}, 5 \mathrm{mmol}, 5$ equiv), and 4-octyne ( $146 \mu \mathrm{L}, 1 \mathrm{mmol}, 1$ equiv) were combined under nitrogen. No dioxane was added to the initial reaction mixture. (E)-2-Hexenezinc iodide S5 in dioxane ([1.26M], $3.9 \mathrm{~mL}, 5 \mathrm{mmol}, 5$ equiv) was added over $4 \mathrm{~h}$. The reaction was quenched with THF, stirred for $0.25 \mathrm{~h}$, and worked up according to the general procedure. Palladium scavenger 1pyrrolidinecarbodithioic acid ammonium salt was added to the drying organic solution. Analysis of the crude reaction mixture via gas chromatography revealed a >95:5 syn:anti ratio. The product was purified by silica gel chromatography ( $0: 100$ to $5: 95$ ethyl acetate : hexanes) followed by reverse phase column chromatography on a Biotage instrument using a SNAP Ultra C18 $60 \mathrm{~g}$ column (1:3 to $0: 1$ water : acetonitrile) to afford 20 as a clear colorless oil (220 mg, 67\%).

${ }^{1} \mathrm{H}$ NMR $\left(400 \mathrm{MHz}, \mathrm{CDCl}_{3}\right) \delta 7.51$ (dd, J = 6.4, 3.1 Hz, 2H), $7.35-7.28(\mathrm{~m}, 3 \mathrm{H}), 5.33-5.15(\mathrm{~m}, 2 \mathrm{H}), 2.15$ $-2.07(\mathrm{~m}, 4 \mathrm{H}), 1.97-1.89(\mathrm{~m}, 2 \mathrm{H}), 1.65(\mathrm{q}, \mathrm{J}=7.4,6.9 \mathrm{~Hz}, 2 \mathrm{H}), 1.59(\mathrm{~d}, \mathrm{~J}=5.6 \mathrm{~Hz}, 3 \mathrm{H}), 1.47-1.36(\mathrm{~m}$, $2 \mathrm{H}), 1.36-1.25(\mathrm{~m}, 2 \mathrm{H}), 1.26-1.13(\mathrm{~m}, 2 \mathrm{H}), 0.91(\mathrm{dt}, \mathrm{J}=14.3,7.3 \mathrm{~Hz}, 6 \mathrm{H}), 0.36(\mathrm{~s}, 6 \mathrm{H})$.

${ }^{13} \mathrm{C}$ NMR $\left(101 \mathrm{MHz}, \mathrm{CDCl}_{3}\right) \delta 153.7,141.5,133.8,131.4,131.0,128.5,127.7,124.9,36.8,34.2,33.4$, $32.9,29.1,24.7,22.4,18.0,14.6,14.5,0.1$.

$\left.{ }^{29} \mathrm{Si} \mathrm{NMR} \mathrm{(119} \mathrm{MHz,} \mathrm{CDCl}_{3}\right) \delta-11.2$.

FTIR $\left(\mathrm{cm}^{-1}\right):$ 2958, 2931, 2870, 1465, 1252, 849, 835, 750.

HRMS (FD) m/z, calcd for [ $\left.\mathrm{C}_{22} \mathrm{H}_{36} \mathrm{Si}\right]^{+}:$328.2586; found: 328.2594 . 


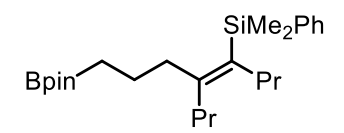

21

Chemical Formula: $\mathrm{C}_{25} \mathrm{H}_{43} \mathrm{BO}_{2} \mathrm{Si}$

Exact Mass: 414.3125

Molecular Weight: 414.5120

(21) According to the general procedure $\mathrm{C}$, (DrewPhos) ${ }_{2} \mathrm{Pdl}_{2}$ (78 $\mathrm{mg}, 0.05$ mmol, $5 \mathrm{~mol} \%$ ), triethylamine (700 $\mu \mathrm{L}, 5 \mathrm{mmol}, 5$ equiv), dimethylphenylsilyl iodide ( $935 \mu \mathrm{L}, 5 \mathrm{mmol}, 5$ equiv), and 4-octyne (146 $\mu \mathrm{L}, 1 \mathrm{mmol}, 1$ equiv) were combined under nitrogen. No dioxane was added to the initial reaction mixture. Alkylzinc iodide $\mathbf{S} 7$ in dioxane ([1.1 M], $4.5 \mathrm{~mL}, 5 \mathrm{mmol}, 5$ equiv) was added over $4 \mathrm{~h}$. The reaction was quenched with THF, stirred for $0.25 \mathrm{~h}$, and worked up according to the general procedure. Palladium scavenger 1pyrrolidinecarbodithioic acid ammonium salt was added to the drying organic solution. Analysis of the crude reaction mixture via gas chromatography revealed a >95:5 syn:anti ratio. The product was purified by silica gel chromatography (hexanes) followed by reverse phase column chromatography on a Biotage instrument using a SNAP Ultra C18 $60 \mathrm{~g}$ column (1:3 to $0: 1$ water : acetonitrile) to afford 21 as a clear colorless oil (228 mg, 55\%).

${ }^{1} \mathrm{H}$ NMR $\left(400 \mathrm{MHz}, \mathrm{CDCl}_{3}\right) \delta 7.52-7.47(\mathrm{~m}, 2 \mathrm{H}), 7.29$ (dd, J = 4.3, $\left.2.1 \mathrm{~Hz}, 3 \mathrm{H}\right), 2.13-2.04(\mathrm{~m}, 4 \mathrm{H}), 1.97$ $-1.90(\mathrm{~m}, 2 \mathrm{H}), 1.47-1.36(\mathrm{~m}, 2 \mathrm{H}), 1.33-1.22(\mathrm{~m}, 4 \mathrm{H}), 1.19(\mathrm{~s}, 12 \mathrm{H}), 0.92(\mathrm{t}, \mathrm{J}=7.4 \mathrm{~Hz}, 3 \mathrm{H}), 0.86(\mathrm{t}, \mathrm{J}$ $=7.3 \mathrm{~Hz}, 3 \mathrm{H}), 0.49(\mathrm{t}, \mathrm{J}=7.9 \mathrm{~Hz}, 2 \mathrm{H}), 0.35(\mathrm{~s}, 6 \mathrm{H})$.

${ }^{13} \mathrm{C} \mathrm{NMR}\left(151 \mathrm{MHz}, \mathrm{CDCl}_{3}\right) \delta 153.9,141.4,133.9,130.8,128.4,127.6,82.9,39.7,34.3,33.3,25.0,24.9$, 24.6, 23.6, 22.4, 14.6, 14.6, 0.2 .

${ }^{11} \mathrm{~B}$ NMR $\left(193 \mathrm{MHz}, \mathrm{CDCl}_{3}\right) \delta 34.2$.

${ }^{29} \mathrm{Si} \mathrm{NMR}\left(119 \mathrm{MHz}, \mathrm{CDCl}_{3}\right) \delta-11.2$.

FTIR $\left(\mathrm{cm}^{-1}\right)$ : 2956, 2930, 2870, 1372, 1318, 1248, 1146, 1109, 834, 815, 770, 728, 700.

HRMS (FD) m/z, calcd for [ $\left.\mathrm{C}_{25} \mathrm{H}_{43} \mathrm{O}_{2} \mathrm{BSi}\right]^{+}: 414.3125$; found: 414.3144.

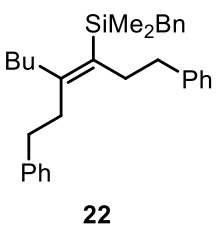

Chemical Formula: $\mathrm{C}_{31} \mathrm{H}_{40} \mathrm{Si}$ Exact Mass: 440.2899

Molecular Weight: 440.7460

(22) According to the general procedure $\mathrm{C}$, (DrewPhos) ${ }_{2} \mathrm{Pdl}_{2}(78 \mathrm{mg}, 0.05 \mathrm{mmol}$, $5 \mathrm{~mol} \%)$, dioxane $(0.5 \mathrm{~mL})$, triethylamine $(700 \mu \mathrm{L}, 5 \mathrm{mmol}, 5$ equiv), dimethylbenzylsilyl iodide $(0.94 \mathrm{~mL}, 5 \mathrm{mmol}, 5$ equiv), and 4-octyne (146 $\mu \mathrm{L}, 1$ mmol, 1 equiv) were combined under nitrogen. $n$-Butylzinc iodide S3 in dioxane ([1.0 M], $5.0 \mathrm{~mL}, 5 \mathrm{mmol}, 5$ equiv) was added over $4 \mathrm{~h}$. The reaction was quenched with THF, stirred for $0.25 \mathrm{~h}$, and worked up according to the general procedure. Palladium scavenger 1-pyrrolidinecarbodithioic acid ammonium salt was added to the drying organic solution. Analysis of the crude reaction mixture via gas chromatography revealed a $>95: 5$ syn:anti ratio. The product was purified by silica gel chromatography (hexanes) followed by reverse phase column chromatography on a Biotage instrument using a SNAP Ultra C18 $60 \mathrm{~g}$ column ( $1: 3$ to $0: 1$ water : acetonitrile) to afford 22 as a colorless oil (238 $\mathrm{mg}, 54 \%)$.

${ }^{1} \mathrm{H}$ NMR $\left(400 \mathrm{MHz}, \mathrm{CDCl}_{3}\right) \delta 7.34-7.27(\mathrm{~m}, 3 \mathrm{H}), 7.25-7.14(\mathrm{~m}, 7 \mathrm{H}), 7.14-7.10(\mathrm{~m}, 2 \mathrm{H}), 7.08-7.03$ $(\mathrm{m}, 1 \mathrm{H}), 7.02-6.98(\mathrm{~m}, 2 \mathrm{H}), 2.73-2.66(\mathrm{~m}, 2 \mathrm{H}), 2.45-2.38(\mathrm{~m}, 2 \mathrm{H}), 2.37-2.31(\mathrm{~m}, 2 \mathrm{H}), 2.30-2.25$ $(\mathrm{m}, 2 \mathrm{H}), 2.24(\mathrm{~s}, 2 \mathrm{H}), 2.21-2.15(\mathrm{~m}, 2 \mathrm{H}), 1.48-1.31(\mathrm{~m}, 4 \mathrm{H}), 0.95(\mathrm{t}, J=7.0 \mathrm{~Hz}, 3 \mathrm{H}), 0.17(\mathrm{~s}, 6 \mathrm{H})$.

${ }^{13} \mathrm{C}$ NMR $\left(101 \mathrm{MHz}, \mathrm{CDCl}_{3}\right) \delta 153.1,142.6,142.5,140.5,131.4,128.5,128.5,128.5,128.4,128.3,128.2$, $126.0,125.9,124.1,37.3,36.8,35.5,34.3,33.7,31.8,27.4,23.4,14.3,-0.6$.

${ }^{29} \mathrm{Si} \mathrm{NMR}\left(119 \mathrm{MHz}, \mathrm{CDCl}_{3}\right) \delta-6.3$.

FTIR $\left(\mathrm{cm}^{-1}\right):$ 3024, 2955, 2929, 2871, 1600, 1493, 1453, 1248, 1205, 1154, 832, 761, 747, 698.

HRMS (LIFDI) m/z, calcd for [ $\mathrm{C}_{31} \mathrm{H}_{40} \mathrm{Si}^{+}$: 440.2899 ; found: 440.2904 . 


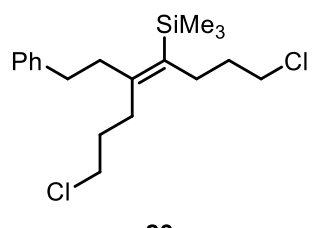

23

Chemical Formula: $\mathrm{C}_{19} \mathrm{H}_{30} \mathrm{Cl}_{2} \mathrm{Si}$ Exact Mass: 356.1494

Molecular Weight: 357.4340

(23) According to the general procedure $\mathrm{C}$, (DrewPhos) ${ }_{2} \mathrm{Pdl}_{2}$ (78 mg, $0.05 \mathrm{mmol}$, $5 \mathrm{~mol} \%)$, dioxane $(0.5 \mathrm{~mL})$, triethylamine ( $700 \mu \mathrm{L}, 5 \mathrm{mmol}, 5$ equiv), trimethylsilyl iodide $(710 \mu \mathrm{L}, 5 \mathrm{mmol}, 5$ equiv), and 1,8-dichloro-oct-4-yne $(178 \mu \mathrm{L}, 1 \mathrm{mmol}, 1$ equiv) were combined under nitrogen. Phenethylzinc iodide S8 in dioxane ([1.2 $\mathrm{M}$ ], $4.2 \mathrm{~mL}, 5 \mathrm{mmol}, 5$ equiv) was added over $4 \mathrm{~h}$. The reaction was quenched with THF, stirred for $0.25 \mathrm{~h}$, and worked up according to the general procedure. Palladium scavenger 1-pyrrolidinecarbodithioic acid ammonium salt was added to the drying organic solution. Analysis of the crude reaction mixture via gas chromatography revealed a $>95: 5$ syn:anti ratio. The product was purified by silica gel chromatography $(0: 100$ to $5: 95$ ethyl acetate : hexanes) followed by reverse phase column chromatography on a Biotage instrument using a SNAP Ultra C18 $60 \mathrm{~g}$ column (3:17 water : acetonitrile) to afford 23 as a clear colorless oil (330 $\mathrm{mg}, 92 \%)$.

${ }^{1} \mathrm{H}$ NMR $\left(400 \mathrm{MHz}, \mathrm{CDCl}_{3}\right) \delta 7.33-7.27(\mathrm{~m}, 2 \mathrm{H}), 7.20(\mathrm{t}, J=6.6 \mathrm{~Hz}, 3 \mathrm{H}), 3.55(\mathrm{dt}, J=12.7,6.5 \mathrm{~Hz}, 4 \mathrm{H})$, $2.72-2.64(\mathrm{~m}, 2 \mathrm{H}), 2.48-2.39(\mathrm{~m}, 2 \mathrm{H}), 2.38-2.31(\mathrm{~m}, 2 \mathrm{H}), 2.28-2.21(\mathrm{~m}, 2 \mathrm{H}), 1.90(\mathrm{dt}, J=14.1,6.5$ $\mathrm{Hz}, 2 \mathrm{H}$ ), 1.72 (dt, $J=14.5,6.6 \mathrm{~Hz}, 2 \mathrm{H}), 0.15$ (s, 9H).

${ }^{13} \mathrm{C} \mathrm{NMR}\left(101 \mathrm{MHz}, \mathrm{CDCl}_{3}\right) \delta 150.2,142.0,134.4,128.6,128.4,126.1,45.3,45.2,38.6,35.8,34.0,32.1$, 29.4, 28.6, 1.3.

${ }^{29} \mathrm{Si} \mathrm{NMR}\left(119 \mathrm{MHz}, \mathrm{CDCl}_{3}\right) \delta-7.1$.

FTIR $\left(\mathrm{cm}^{-1}\right): 3027,2954,1602,1496,1454,1307,1249,836,755,699$.

HRMS (LIFDI) m/z, calcd for [ $\left.\mathrm{C}_{19} \mathrm{H}_{30} \mathrm{Cl}_{2} \mathrm{Si}\right]^{+}:$356.1494; found: 356.1477 .

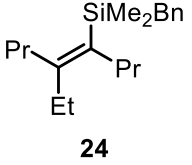

Chemical Formula: $\mathrm{C}_{19} \mathrm{H}_{32} \mathrm{Si}$

Molecular Weight: 288.5500 Exact Mass: 288.2273

(24) According to the general procedure $\mathrm{C}$, (JessePhos) ${ }_{2} \mathrm{PdCl}_{2}(111 \mathrm{mg}, 0.1 \mathrm{mmol}$, $5 \mathrm{~mol} \%)$, dioxane $(1 \mathrm{~mL})$, triethylamine $(1.4 \mathrm{~mL}, 10 \mathrm{mmol}, 5$ equiv), dimethylbenzylsilyl iodide (1.80 mL, $10 \mathrm{mmol}, 5$ equiv), and 4-octyne $(293 \mu \mathrm{L}, 2$ $\mathrm{mmol}, 1$ equiv) were combined under nitrogen. Ethylzinc iodide $\mathbf{S 2}$ in dioxane ([1.6 $\mathrm{M}$ ], $6.25 \mathrm{~mL}, 10 \mathrm{mmol}, 5$ equiv) was added over $4 \mathrm{~h}$. The reaction was worked up according to the general procedure. Analysis of the crude reaction mixture via gas chromatography revealed a $<5: 95$ syn:anti ratio. The product was purified by silica gel chromatography (hexanes) followed by reverse phase column chromatography on a Biotage instrument using a SNAP Ultra C18 $60 \mathrm{~g}$ column (3:17 water : acetonitrile) to afford 24 as a colorless oil (532 mg, $92 \%)$.

${ }^{1} \mathrm{H}$ NMR $\left(600 \mathrm{MHz}, \mathrm{CDCl}_{3}\right) \delta 7.18(\mathrm{t}, J=7.7 \mathrm{~Hz}, 2 \mathrm{H}), 7.08-7.02(\mathrm{~m}, 1 \mathrm{H}), 7.02-6.97(\mathrm{~m}, 2 \mathrm{H}), 2.19(\mathrm{~s}$, $2 \mathrm{H}), 2.11(\mathrm{q}, J=7.5 \mathrm{~Hz}, 2 \mathrm{H}), 2.08-2.02(\mathrm{~m}, 2 \mathrm{H}), 1.97-1.89(\mathrm{~m}, 2 \mathrm{H}), 1.47-1.30(\mathrm{~m}, 2 \mathrm{H}), 1.24-1.07$ $(\mathrm{m}, 2 \mathrm{H}), 0.98$ (t, $J=7.5 \mathrm{~Hz}, 3 \mathrm{H}), 0.91(\mathrm{t}, J=7.3 \mathrm{~Hz}, 3 \mathrm{H}), 0.85$ (t, $J=7.3 \mathrm{~Hz}, 3 \mathrm{H}), 0.08(\mathrm{~s}, 6 \mathrm{H})$.

${ }^{13} \mathrm{C}$ NMR $\left(151 \mathrm{MHz}, \mathrm{CDCl}_{3}\right) \delta 154.5,140.8,130.8,128.4,128.1,123.9,38.5,34.1,27.5,24.5,24.2,22.7$, 14.6, 14.5, 13.8, -0.8 .

${ }^{29} \mathrm{Si} \mathrm{NMR}\left(119 \mathrm{MHz}, \mathrm{CDCl}_{3}\right) \delta-6.7$.

FTIR (cm-1): 2959, 2930, 2871, 1600, 1493, 1453, 1249, 1206, 1154, 829, 761, 698, 477.

HRMS (ESI) m/z, calcd for [ $\left.{ }_{16} \mathrm{H}_{27} \mathrm{OSi}\right]^{+}: 288.2273$; found: 288.2274 . 


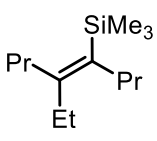

25

Chemical Formula: $\mathrm{C}_{13} \mathrm{H}_{28} \mathrm{Si}$ Exact Mass: 212.1960 Molecular Weight: 212.4520

(25) According to the general procedure $\mathrm{C}$, (JessePhos) ${ }_{2} \mathrm{PdCl}_{2}$ (111 mg, $0.1 \mathrm{mmol}$, $5 \mathrm{~mol} \%)$, dioxane $(1 \mathrm{~mL})$, triethylamine $(1.4 \mathrm{~mL}, 10 \mathrm{mmol}, 5$ equiv), trimethylsilyl iodide ( $1.40 \mathrm{~mL}, 10 \mathrm{mmol}, 5$ equiv), and 4-octyne (293 $\mu \mathrm{L}, 2 \mathrm{mmol}, 1$ equiv) were combined under nitrogen. Ethylzinc iodide $\mathbf{S 2}$ in dioxane ([1.6 M], $6.25 \mathrm{~mL}, 10$ mmol, 5 equiv) was added over $4 \mathrm{~h}$. The reaction was worked up according to the general procedure. Analysis of the crude reaction mixture via gas chromatography revealed an 8:92 syn:anti ratio. The product was purified by silica gel chromatography (hexanes) followed by reverse phase column chromatography on a Biotage instrument using a SNAP Ultra C18 $60 \mathrm{~g}$ column (3:17 water : acetonitrile) to afford 25 as a clear colorless oil (318 $\mathrm{mg}, 75 \%)$.

${ }^{1} \mathrm{H} \mathrm{NMR}\left(600 \mathrm{MHz}, \mathrm{CDCl}_{3}\right) \delta 2.15-2.05(\mathrm{~m}, 4 \mathrm{H}), 2.04-1.98(\mathrm{~m}, 2 \mathrm{H}), 1.46-1.31(\mathrm{~m}, 2 \mathrm{H}), 1.30-1.15(\mathrm{~m}$, $2 \mathrm{H}$ ), 0.96 (t, $J=7.5 \mathrm{~Hz}, 3 \mathrm{H}), 0.90$ (dt, $J=9.4,7.3 \mathrm{~Hz}, 6 \mathrm{H}), 0.12(\mathrm{~s}, 9 \mathrm{H})$.

${ }^{13} \mathrm{C} \mathrm{NMR}\left(151 \mathrm{MHz}, \mathrm{CDCl}_{3}\right) \delta 153.4,132.7,38.5,34.1,24.6,24.2,22.8,14.5,14.4,13.8,1.4$.

${ }^{29} \mathrm{Si} \mathrm{NMR}\left(119 \mathrm{MHz}, \mathrm{CDCl}_{3}\right) \delta-7.8$.

FTIR $\left(\mathrm{cm}^{-1}\right):$ 2960, 2931, 2872, 1599, 1465, 1248, 849, 834, 758.

HRMS (ESI) m/z, calcd for [ $\left.\mathrm{C}_{16} \mathrm{H}_{27} \mathrm{OSi}\right]^{+}: 213.2033$; found: 213.1842 .

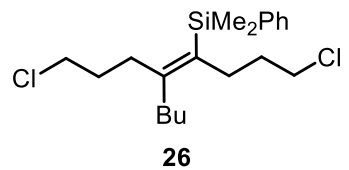

Chemical Formula: $\mathrm{C}_{20} \mathrm{H}_{32} \mathrm{Cl}_{2} \mathrm{Si}$

Exact Mass: 370.1650

Molecular Weight: 371.4610
(26) According to the general procedure $\mathrm{C}$, (JessePhos) ${ }_{2} \mathrm{PdCl}_{2}$ (56 mg, 0.05 $\mathrm{mmol}, 5 \mathrm{~mol} \%$ ), dioxane ( $0.5 \mathrm{~mL})$, Et ${ }_{3} \mathrm{~N}\left(700 \mu \mathrm{L}, 5 \mathrm{mmol}, 5\right.$ equiv), $\mathrm{Me}_{2} \mathrm{PhSil}$ (935 $\mu \mathrm{L}, 5 \mathrm{mmol}, 5$ equiv), and 1,8-dichloro-oct-4-yne (178 $\mu \mathrm{L}, 1 \mathrm{mmol}, 1$ equiv) were combined under nitrogen. $n$-Butylzinc iodide $\mathbf{S} 3$ in dioxane ([2.0 M], 2.5 $\mathrm{mL}, 5 \mathrm{mmol}, 5$ equiv) was added over $4 \mathrm{~h}$. The reaction was quenched with THF, stirred for $0.25 \mathrm{~h}$, and worked up according to the general procedure. Analysis of the crude reaction mixture via gas chromatography revealed a 17:83 syn:anti ratio. The product was purified by silica gel chromatography $(0: 100$ to $5: 95$ ethyl acetate : hexanes) followed by reverse phase column chromatography on a Biotage instrument using a SNAP Ultra C18 $60 \mathrm{~g}$ column ( $3: 17$ water : acetonitrile) to afford 26 as a clear colorless oil $(221 \mathrm{mg}, 60 \%)$.

${ }^{1} \mathrm{H}$ NMR $\left(400 \mathrm{MHz}, \mathrm{CDCl}_{3}\right) \delta 7.6-7.4(\mathrm{~m}, 2 \mathrm{H}), 7.4-7.3(\mathrm{~m}, 3 \mathrm{H}), 3.5(\mathrm{t}, J=6.5 \mathrm{~Hz}, 2 \mathrm{H}), 3.1(\mathrm{t}, J=6.8 \mathrm{~Hz}$, $2 \mathrm{H}), 2.4-2.2(\mathrm{~m}, 2 \mathrm{H}), 2.2-2.0(\mathrm{~m}, 4 \mathrm{H}), 1.8-1.7(\mathrm{~m}, 2 \mathrm{H}), 1.6-1.5(\mathrm{~m}, 2 \mathrm{H}), 1.4(\mathrm{td}, J=3.7,1.9 \mathrm{~Hz}, 4 \mathrm{H})$, $1.0-0.8(\mathrm{~m}, 3 \mathrm{H}), 0.4(\mathrm{~s}, 6 \mathrm{H})$.

${ }^{13} \mathrm{C}$ NMR $\left(101 \mathrm{MHz}, \mathrm{CDCl}_{3}\right) \delta 153.5,140.7,133.7,130.8,128.9,127.9,45.3,44.9,34.4,34.0,32.0,31.4$, $31.1,29.4,23.2,14.2,-0.1$.

$\left.{ }^{29} \mathrm{Si} \mathrm{NMR} \mathrm{(119} \mathrm{MHz,} \mathrm{CDCl}_{3}\right) \delta-10.9$.

FTIR $\left(\mathrm{cm}^{-1}\right): 2956,2871,1595,1457,1428,1305,1250,1109,832,814,772,730,701$.

HRMS (FD) m/z, calcd for [ $\left.\mathrm{C}_{19} \mathrm{H}_{30} \mathrm{Cl}_{2} \mathrm{Si}^{+}\right]^{+}: 370.1650$; found: 370.1639 . 


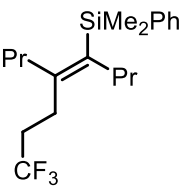

27

Chemical Formula: $\mathrm{C}_{19} \mathrm{H}_{29} \mathrm{~F}_{3} \mathrm{Si}$

Exact Mass: 342.1991

Molecular Weight: 342.5212

(27) According to the general procedure $\mathrm{C}$, (JessePhos) ${ }_{2} \mathrm{PdCl}_{2}$ (56 mg, 0.05 $\mathrm{mmol}, 5 \mathrm{~mol} \%)$, dioxane $(0.5 \mathrm{~mL})$, triethylamine $(700 \mu \mathrm{L}, 5 \mathrm{mmol}, 5$ equiv), dimethylphenylsilyl iodide ( $940 \mu \mathrm{L}, 5 \mathrm{mmol}, 5$ equiv), and 4-octyne (146 $\mu \mathrm{L}, 1$ mmol, 1 equiv) were combined under nitrogen. 3,3,3-trifluorozinc iodide $\mathbf{S 9}$ in dioxane ([1.2M], $4.1 \mathrm{~mL}, 5 \mathrm{mmol}, 5$ equiv) was added over $4 \mathrm{~h}$. The reaction was quenched with THF, stirred for $0.25 \mathrm{~h}$, and worked up according to the general procedure. Analysis of the crude reaction mixture via gas chromatography revealed a $<5: 95$ syn:anti ratio. The product was purified by silica gel chromatography ( $0: 100$ to $5: 95$ ethyl acetate : hexanes) followed by reverse phase column chromatography on a Biotage instrument using a SNAP Ultra C18 $60 \mathrm{~g}$ column (1:3 to $0: 1$ water : acetonitrile) to afford 27 as a clear colorless oil (208 $\mathrm{mg}, 61 \%)$.

${ }^{1} \mathrm{H}$ NMR $\left(400 \mathrm{MHz}, \mathrm{CDCl}_{3}\right) \delta 7.50-7.45(\mathrm{~m}, 2 \mathrm{H}), 7.32(\mathrm{dd}, J=4.7,1.9 \mathrm{~Hz}, 3 \mathrm{H}), 2.46-2.26(\mathrm{~m}, 2 \mathrm{H}), 2.23$ $-2.02(\mathrm{~m}, 4 \mathrm{H}), 1.99-1.80(\mathrm{~m}, 2 \mathrm{H}), 1.39-1.23(\mathrm{~m}, 2 \mathrm{H}), 1.23-1.07(\mathrm{~m}, 2 \mathrm{H}), 0.90(\mathrm{t}, J=7.3 \mathrm{~Hz}, 3 \mathrm{H})$, $0.63(\mathrm{t}, J=7.3 \mathrm{~Hz}, 3 \mathrm{H}), 0.37(\mathrm{~s}, 6 \mathrm{H})$.

${ }^{13} \mathrm{C} \mathrm{NMR}\left(101 \mathrm{MHz}, \mathrm{CDCl}_{3}\right) \delta 149.4,140.7,133.7,134.0,128.7,127.8,127.1$ (q, $\left.J=276.9 \mathrm{~Hz}\right), 38.9$, $34.2,33.4$ (q, $J=28.0 \mathrm{~Hz}$ ), 24.5, $23.4(\mathrm{q}, J=2.8 \mathrm{~Hz}$ ), 22.2, 14.5, 14.0, -0.1.

${ }^{29} \mathrm{Si} \mathrm{NMR}\left(119 \mathrm{MHz}, \mathrm{CDCl}_{3}\right) \delta-10.7$.

${ }^{19} \mathrm{~F} \mathrm{NMR}\left(565 \mathrm{MHz}, \mathrm{CDCl}_{3}\right) \delta-66.9$.

FTIR (cm-1): 2960, 2932, 2873, 1428, 1377, 1302, 1259, 1249, 1139, 1111, 1072, 978, 835, 813, 772, 729, 701.

HRMS (LIFDI) m/z, calcd for [ $\left.\mathrm{C}_{19} \mathrm{H}_{30} \mathrm{Cl}_{2} \mathrm{Si}\right]^{+}: 342.199$; found: 342.1983 .

\section{Synthesis of Tetrasubstituted Alkenes via Hiyama Cross-Coupling}

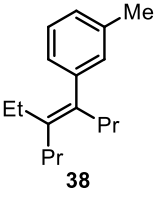
Exact Mass: 230.2035
Chemical Formula: $\mathrm{C}_{17} \mathrm{H}_{26}$ Molecular Weight: 230.3950

(38) A $25 \mathrm{~mL}$ Schlenk flask equipped with a magnetic stirbar and rubber septum was flame-dried, allowed to cool to room temperature under vacuum, and refilled with $\mathrm{N}_{2}$. The flask was briefly opened, charged with potassium trimethylsilanolate $(257 \mathrm{mg}, 2$ mmol, 2 equiv) and 18-crown-6 (528 mg, $2 \mathrm{mmol}, 2$ equiv) and the septum was replaced. The flask was evacuated and refilled with $\mathrm{N}_{2} 3$ times. THF $(2 \mathrm{~mL})$ and vinylsilane 16 ( $315 \mathrm{uL}, 1 \mathrm{mmol}, 1$ equiv) were added by syringe. The reaction was stirred at $65{ }^{\circ} \mathrm{C}$ for $0.5 \mathrm{~h}$. At this time, a solution of [(allyl)PdCl] $2(9 \mathrm{mg}, 0.025 \mathrm{mmol}$, $2.5 \mathrm{~mol} \%$ ), SPhos ( $21 \mathrm{mg}, 0.05 \mathrm{mmol}, 5 \mathrm{~mol} \%$ ), and 3-chlorotoluene (177 uL, 1.5 mmol, 1.5 equiv) in THF (2 mL) was added by syringe. The reaction was allowed to stir at $65^{\circ} \mathrm{C}$ for $18 \mathrm{~h}$. The reaction was then cooled to room temperature, opened to air, quenched with brine $(5 \mathrm{~mL})$, and diluted with $\mathrm{Et}_{2} \mathrm{O}(5 \mathrm{~mL})$. The aqueous layer was extracted with $\mathrm{Et}_{2} \mathrm{O}(3 \times 3 \mathrm{~mL})$. The combined organic layers were washed with brine $(10 \mathrm{~mL})$, dried over $\mathrm{MgSO}_{4}$, filtered, and concentrated in vacuo. The crude material was purified via silica column chromatography (hexanes) to yield alkene 38 as a clear oil (142 mg, 62\%).

${ }^{1} \mathrm{H}$ NMR $\left(600 \mathrm{MHz}, \mathrm{CDCl}_{3}\right) \delta 7.17(\mathrm{t}, J=7.5 \mathrm{~Hz}, 1 \mathrm{H}), 7.01(\mathrm{~d}, J=7.5 \mathrm{~Hz}, 1 \mathrm{H}), 6.88-6.85(\mathrm{~m}, 2 \mathrm{H}), 2.34(\mathrm{~s}$, $3 \mathrm{H}), 2.28-2.24(\mathrm{~m}, 2 \mathrm{H}), 2.17-2.12(\mathrm{~m}, 2 \mathrm{H}), 1.82(\mathrm{q}, J=7.5 \mathrm{~Hz}, 2 \mathrm{H}), 1.47(\mathrm{dq}, J=15.0,7.4 \mathrm{~Hz}, 2 \mathrm{H}), 1.30$ $-1.19(\mathrm{~m}, 2 \mathrm{H}), 0.97(\mathrm{t}, J=7.3 \mathrm{~Hz}, 3 \mathrm{H}), 0.85$ (q, $J=7.3 \mathrm{~Hz}, 6 \mathrm{H})$.

${ }^{13} \mathrm{C}$ NMR $\left(101 \mathrm{MHz}, \mathrm{CDCl}_{3}\right) \delta 144.3,137.3,137.2,136.0,129.6,127.7,126.5,126.1,36.4,32.6,25.9$, 22.4, 21.7, 14.6, 14.2, 13.9.

FTIR $\left(\mathrm{cm}^{-1}\right): 2961,2932,2871,1463,1377,1053,784,715$.

HRMS (ESI) m/z, calcd for [ $\left.\mathrm{C}_{19} \mathrm{H}_{30} \mathrm{Cl}_{2} \mathrm{Si}\right]^{+}:$230.2035; found: 230.2035 . 


\section{Additional Optimization Data}

Note: All reactions in this section were performed on a $0.25 \mathrm{mmol}$ scale in a nitrogen filled glovebox.

\section{Examination of Addition of Reagents in the Multicomponent Carbosilylation Reaction of Diaryl Alkynes:}

In a nitrogen filled glovebox, a 1-dram vial equipped with a magnetic stirbar was charged with [Pd] (2 mol $\%)$, diphenylacetylene (1 equiv), dioxane $(125 \mu \mathrm{L})$, diethylzinc (1 equiv) and silyl-iodide (1 equiv). The vial was sealed with a septum cap and removed from the glovebox. The reaction was quenched with diethyl ether $(1 \mathrm{~mL})$ and water $(1 \mathrm{~mL})$ via syringe. 1,3,5-trimethoxybenzene (TMB) (0.33 equiv) and nonane (1 equiv) were added as an NMR standard and a GC standard, respectively. An aliquot of the organic layer was then filtered through $\mathrm{MgSO}_{4}$ and silica gel for NMR and GC analysis.

Table S1: Examination of Order of Addition and Slow Addition:

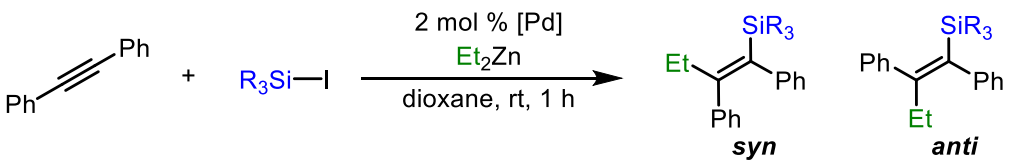

\begin{tabular}{|c|c|c|c|c|c|c|c|}
\hline Entry & [Pd] Source & $\begin{array}{l}\text { Silyl lodide } \\
\text { (equiv) }\end{array}$ & $\begin{array}{l}\text { Nucleophile } \\
\text { (equiv) }\end{array}$ & $\begin{array}{c}\mathrm{Et}_{3} \mathrm{~N} \\
\text { (equiv) }\end{array}$ & Conditions & $\underset{a}{\text { Yield }}$ & $\underset{b}{\text { syn:anti }}$ \\
\hline 1 & None & TMSI (1) & $\mathrm{Et}_{2} \mathrm{Zn}(1)$ & 0 & $\begin{array}{l}\text { TMSI added last, } \\
\text { over } 0.5 \mathrm{~min}\end{array}$ & trace & $>95: 5$ \\
\hline 2 & None & $\mathrm{PhMe}_{2} \mathrm{Sil}(1)$ & $\mathrm{Et}_{2} \mathrm{Zn}(1)$ & 0 & $\begin{array}{l}\mathrm{PhMe}_{2} \mathrm{Sil} \text { added last, } \\
\text { over } 0.5 \mathrm{~min}\end{array}$ & $<1$ & $>95: 5$ \\
\hline 3 & $\left(\mathrm{Ph}_{3} \mathrm{P}\right)_{4} \mathrm{Pd}$ & TMSI (1) & $\mathrm{Et}_{2} \mathrm{Zn}(1)$ & 0 & $\begin{array}{l}\text { TMSI added last, } \\
\text { over } 0.5 \text { min }\end{array}$ & 6 & $>95: 5$ \\
\hline 4 & $\left(\mathrm{Ph}_{3} \mathrm{P}\right)_{4} \mathrm{Pd}$ & TMSI (1) & $\mathrm{Et}_{2} \mathrm{Zn}(1)$ & 0 & $\begin{array}{l}\mathrm{Et}_{2} \mathrm{Zn} \text { added last, } \\
\text { over } 0.5 \mathrm{~min}\end{array}$ & 5 & $>95: 5$ \\
\hline 5 & $\left(\mathrm{Ph}_{3} \mathrm{P}\right)_{4} \mathrm{Pd}$ & $\mathrm{PhMe}_{2} \mathrm{Sil}(1)$ & $\mathrm{Et}_{2} \mathrm{Zn}(1)$ & 0 & $\begin{array}{l}\mathrm{Et}_{2} \mathrm{Zn} \text { added last, } \\
\text { over } 0.5 \mathrm{~min}\end{array}$ & 6 & $>95: 5$ \\
\hline 6 & $\left(\mathrm{Ph}_{3} \mathrm{P}\right)_{4} \mathrm{Pd}$ & $\mathrm{PhMe}_{2} \mathrm{Sil}(1)$ & $\mathrm{Et}_{2} \mathrm{Zn}(1)$ & 0 & $\begin{array}{c}\mathrm{Et}_{2} \mathrm{Zn} \text { added last, } \\
\text { over } 1 \mathrm{~h}\end{array}$ & 25 & $>95: 5$ \\
\hline 7 & $\left(\mathrm{Ph}_{3} \mathrm{P}\right)_{2} \mathrm{PdCl}_{2}$ & $\mathrm{PhMe}_{2} \mathrm{Sil}(1)$ & $\mathrm{Et}_{2} \mathrm{Zn}(1)$ & 0 & $\begin{array}{c}\mathrm{Et}_{2} \mathrm{Zn} \text { added last, } \\
\text { over } 1 \mathrm{~h}\end{array}$ & 70 & $>95: 5$ \\
\hline 8 & $\left(\mathrm{Ph}_{3} \mathrm{P}\right)_{2} \mathrm{PdCl}_{2}$ & $\mathrm{PhMe}_{2} \mathrm{Sil}$ (1) & $\mathrm{Et}_{2} \mathrm{Zn}(1)$ & 0 & $\begin{array}{c}\text { Et } 2 \text { Zn added last, } \\
\text { over } 4 \mathrm{~h}\end{array}$ & 89 & $>95: 5$ \\
\hline 9 & $\left(\mathrm{Ph}_{3} \mathrm{P}\right)_{2} \mathrm{PdCl}_{2}$ & $\mathrm{PhMe}_{2} \mathrm{Sil}(1)$ & $\mathrm{EtZnI}(1)$ & 1 & $\begin{array}{l}\text { EtZnl added last, } \\
\text { over } 4 \mathrm{~h}\end{array}$ & 89 & $>95: 5$ \\
\hline 10 & $\left(\mathrm{Ph}_{3} \mathrm{P}\right)_{2} \mathrm{PdCl}_{2}$ & $\mathrm{PhMe}_{2} \mathrm{Sil}(1)$ & BuZnl (1) & 1 & $\begin{array}{l}\text { BuZnl added last, } \\
\text { over } 4 \mathrm{~h}\end{array}$ & 95 & $>95: 5$ \\
\hline 11 & $\left(\mathrm{Ph}_{3} \mathrm{P}\right)_{2} \mathrm{PdCl} 2$ & $\mathrm{PhMe}_{2} \mathrm{Sil}(3)$ & EtZnl (1.5) & 3 & $\begin{array}{l}\text { EtZnl added last, } \\
\text { over } 4 \mathrm{~h}\end{array}$ & 95 & $>95: 5$ \\
\hline
\end{tabular}




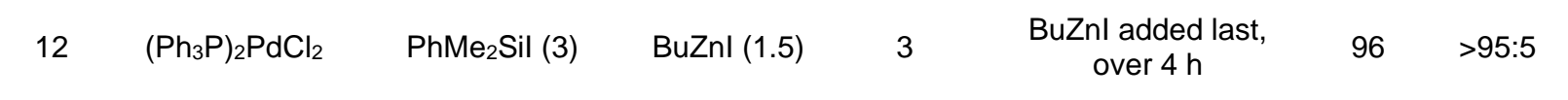

${ }^{a}$ Yields obtained by ${ }^{1} \mathrm{H}$ NMR with TMB as an internal standard. ${ }^{b}$ Ratio determined by GC.

\section{Examination of Ligands in the Multicomponent Carbosilylation Reaction of Dialkyl Alkynes:}

In a nitrogen filled glovebox, a 1-dram vial equipped with a magnetic stirbar was charged with $\mathrm{Pd}_{2} \mathrm{dba}_{3}$ $\mathrm{CHCl}_{3}(2.5 \mathrm{~mol} \%)$, ligand (10 mol \%), dioxane (125 uL), triethylamine (3 equiv), 4-octyne (1 equiv), and dimethylphenylsilyl iodide (1 equiv). The vial was sealed with a septum cap and removed from the glovebox. A solution of ethylzinc iodide (1.0 equiv) in dioxane was then added dropwise over $4 \mathrm{~h}$ via syringe pump, with stirring. The reaction was quenched with diethyl ether $(1 \mathrm{~mL})$ and water $(1 \mathrm{~mL})$ via syringe. 1,3,5trimethoxybenzene (TMB) ( 0.33 equiv) and nonane ( 1 equiv) were added as an NMR standard and a GC standard, respectively. An aliquot of the organic layer was then filtered through $\mathrm{MgSO}_{4}$ and silica gel for NMR and GC analysis.

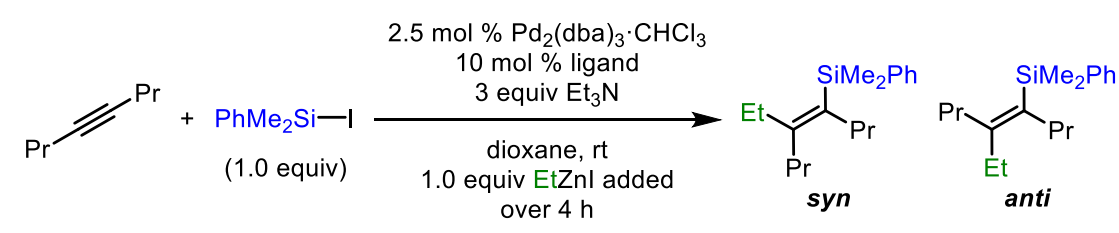

\begin{tabular}{cccc} 
Entry & Ligand & Yield $^{a}$ & syn:anti \\
\hline 1 & $\mathrm{Ph}_{3} \mathrm{P}$ & 88 & $65: 35$ \\
2 & $\left(3,5-\mathrm{CF}_{3} \mathrm{C}_{6} \mathrm{H}_{3}\right)_{3} \mathrm{P}$ & 13 & $50: 50$ \\
3 & $\left(4-\mathrm{MeOC}_{6} \mathrm{H}_{4}\right)_{3} \mathrm{P}$ & 66 & $60: 40$ \\
4 & DrewPhos & 76 & $90: 10$ \\
5 & $(o-\text { tol })_{3} \mathrm{P}$ & 65 & $18: 82$ \\
6 & $\mathrm{Ph}_{2} \mathrm{P}$ Bu & 60 & $3: 97$ \\
7 & JessePhos & 67 & $5: 95$
\end{tabular}

aYields obtained by ${ }^{1} \mathrm{H}$ NMR with TMB as an internal standard. ${ }^{b}$ Ratio determined by GC.

\section{Further Optimization of Conditions for the Multicomponent Carbosilylation Reaction of Dialkyl Alkynes:}

In a nitrogen filled glovebox, a 1-dram vial equipped with a magnetic stirbar was charged with palladium precatalyst, dioxane (125 uL), triethylamine, 4-octyne (1 equiv), and dimethylphenylsilyl iodide. The vial was sealed with a septum cap and removed from the glovebox. A solution of ethylzinc iodide in dioxane was then added dropwise over $4 \mathrm{~h}$ via syringe pump, with stirring. The reaction was quenched with diethyl ether $(1 \mathrm{~mL})$ and water $(1 \mathrm{~mL})$ via syringe. 1,3,5-trimethoxybenzene (TMB) (0.33 equiv) and nonane (1 equiv) were added as an NMR standard and a GC standard, respectively. An aliquot of the organic layer was then filtered through $\mathrm{MgSO}_{4}$ and silica gel for NMR and GC analysis.

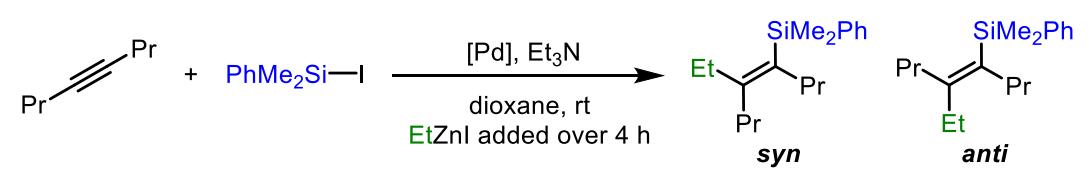




\begin{tabular}{|c|c|c|c|c|c|c|}
\hline Entry & {$[\mathrm{Pd}](\mathrm{mol} \%)$} & $\begin{array}{c}\mathrm{Me}_{2} \mathrm{PhSil} \\
\text { (equiv) }\end{array}$ & $\mathrm{Et}_{3} \mathrm{~N}$ (equiv) & $\begin{array}{l}\text { EtZnl } \\
\text { (equiv) }\end{array}$ & Yield (\%) & syn:anti ${ }^{b}$ \\
\hline 1 & $\begin{array}{c}\text { (DrewPhos) }{ }_{2} \mathrm{Pdl}_{2} \\
(2 \mathrm{~mol} \%)\end{array}$ & 3 & 3 & 1.5 & 53 & $>95: 5$ \\
\hline 2 & $\begin{array}{c}\text { (DrewPhos) })_{2} \mathrm{Pdl}_{2} \\
(5 \mathrm{~mol} \%)\end{array}$ & 3 & 3 & 1.5 & 60 & $>95: 5$ \\
\hline 3 & $\begin{array}{c}\text { (DrewPhos) })_{2} \mathrm{Pdl}_{2} \\
(5 \mathrm{~mol} \%)\end{array}$ & 5 & 5 & 1.5 & 65 & $>95: 5$ \\
\hline 4 & $\begin{array}{c}\text { (DrewPhos) })_{2} \mathrm{Pdl}_{2} \\
(5 \mathrm{~mol} \%)\end{array}$ & 5 & 5 & 2 & 74 & $>95: 5$ \\
\hline 5 & $\begin{array}{c}\text { (DrewPhos) })_{2} \mathrm{Pdl}_{2} \\
(5 \mathrm{~mol} \%)\end{array}$ & 5 & 5 & 5 & 99 & $>95: 5$ \\
\hline 6 & $\begin{array}{c}\text { (JessePhos })_{2} \mathrm{Pdl}_{2} \\
(5 \mathrm{~mol} \%)\end{array}$ & 5 & 5 & 5 & 94 & $<5: 95$ \\
\hline
\end{tabular}

aYields obtained by ${ }^{1} \mathrm{H}$ NMR with TMB as an internal standard. ${ }^{b}$ Ratio determined by GC.

\section{Examination of Silicon Substitution}

In a nitrogen filled glovebox, a 1-dram vial equipped with a magnetic stirbar was charged with $\left(\mathrm{Ph}_{3} \mathrm{P}\right)_{2} \mathrm{PdCl}_{2}$ (3.5 mg, $0.005 \mathrm{mmol}, 2 \mathrm{~mol} \%$ ), 1,2-bis(4-methoxyphenyl)acetylene (59 mg, $0.25 \mathrm{mmol}, 1$ equiv), dioxane $(125 \mu \mathrm{L})$, and silyl-iodide $(0.75 \mathrm{mmol}, 3$ equiv). The vial was sealed with a septum cap and removed from the glovebox. Alkylzinc iodide $\mathbf{S} 7(0.6 \mathrm{M}, 0.68 \mathrm{~mL}, 0.38 \mathrm{mmol}, 1.5$ equiv) was added over $4 \mathrm{~h}$ via syringe pump. The reaction was quenched with diethyl ether $(1 \mathrm{~mL})$ and water $(1 \mathrm{~mL})$ via syringe. 1,3,5trimethoxybenzene (TMB) (14 mg, 0.33 equiv) and nonane (32 mg, 1 equiv) were added as an NMR standard and a GC standard, respectively. An aliquot of the organic layer was then filtered through $\mathrm{MgSO}_{4}$ and silica gel for NMR and GC analysis.
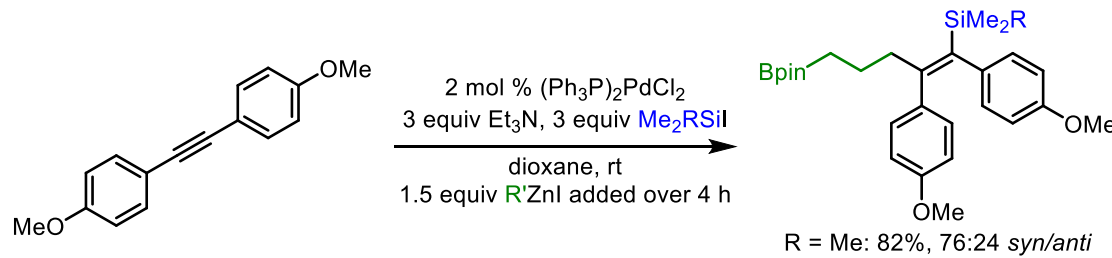

$\mathrm{R}=\mathrm{Ph}: 97 \%, 50: 50$ syn/ant

\section{References}

(1) Pangborn, A. B.; Giardello, M. A.; Grubbs, R. H.; Rosen, R. K.; Timmers, F. J. Organometallics 1996, 15,1518

(2) McAtee, J. R.; Krause, S. B.; Watson, D. A. Adv. Synth. Catal. 2015, 357, 2317.

(3) Cinderella, A. P.; Vulovic, B.; Watson, D. A. J. Am. Chem. Soc. 2017, $139,7741$.

(4) McAtee, J. R.; Yap, G. P. A.; Watson, D. A. J. Am. Chem. Soc. 2014, 136, 10166.

(5) Reid, W. B.; Spillane, J. J.; Krause, S. B.; Watson, D. A. J. Am. Chem. Soc. 2016, 138, 5539.

(6) Pan Y.; Young, G. B.; J. Organomet. Chem. 1999, 577, 257.

(7) Clarke, M. L.; Elleis, D.; Mason, K. L.; Orpen, A. G.; Pringle, P. G.; Wingad, R. L.; Zaher, D. A.; 
Barker, R. T. Dalton Trans., 2005, 1294.

(8) Adachi, Y.; Kamei, N.; Yokoshima, S.; Fukuyama, T. Org. Lett. 2011, 13, 4446.

(9) Levin, V. V.; Zemtsov, A. A.; Struchkova, M. I.; Dilman A. D. Org Lett. 2013, 15, 917.

(10) Hoang, C. T.; Alezra, V.; Guillot, R.; Kouklovsky, C. Org. Lett. 2007, 9, 2521.

(11) Guo, W.; Pleixats, R.; Shafir, A.; Parella T. Adv. Synth. Catal,. 2015, 357, 89.

(12) Tang, Q.; Xia, D.; Jin, X.; Zhang, Q.; Sun, X.-Q.; Wang, C. J. Amer. Chem. Soc. 2013, 135, 4628.

(13) Mio, M. J.; Kopel, L. C.; Braun, J. B.; Gadzikwa, T. L.; Hull, K. L.; Brisbois, R. G.; Markworth, C. J.; Grieco, P. A. Org. Lett. 2002, 4, 3199.

\section{Spectral Data}




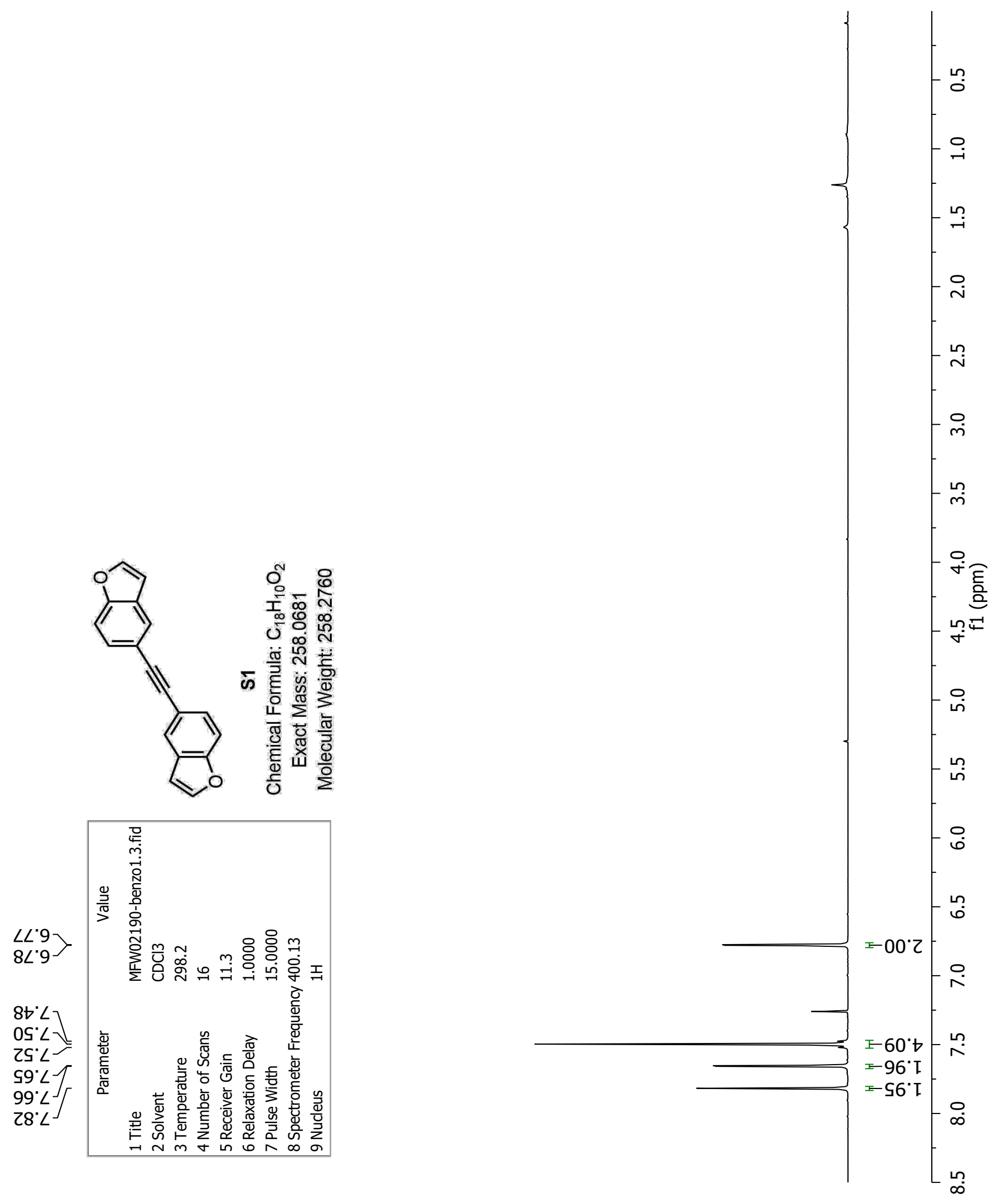




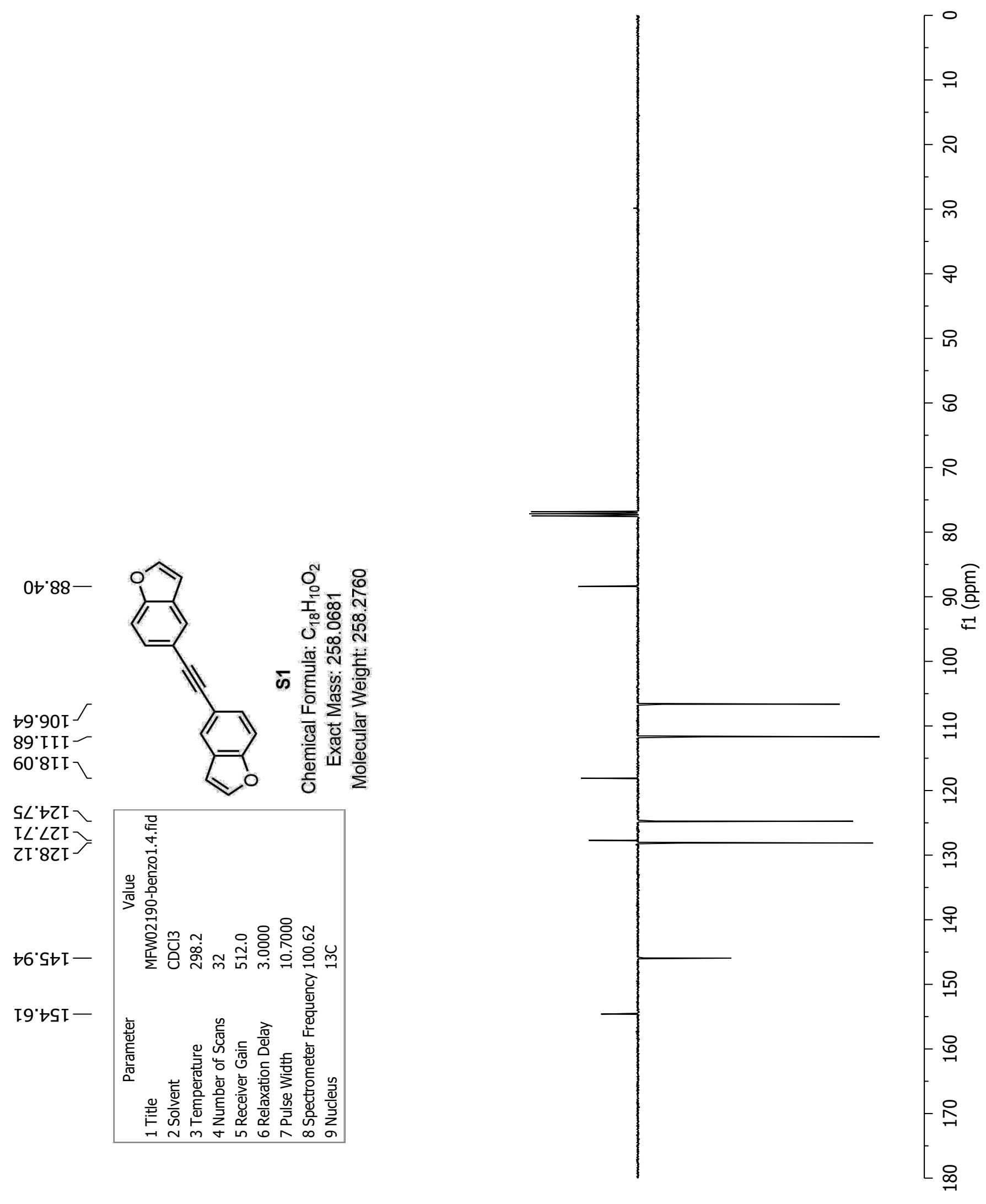



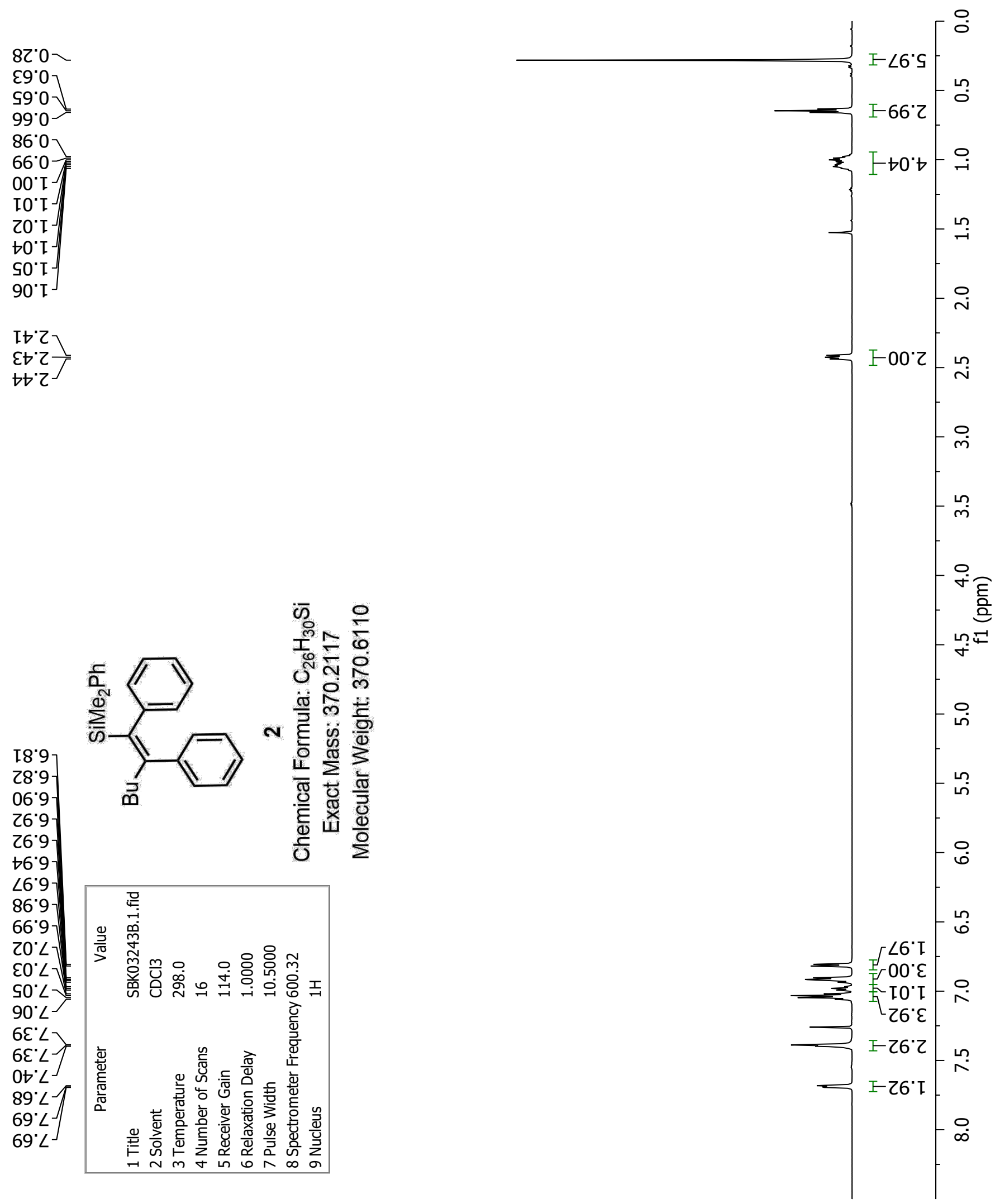
ع $0^{\circ} 0^{--}$

$66^{\circ} \varepsilon I-$

ะ8'てて-

$Z \angle L^{\circ} 0 \varepsilon-$

$99^{\circ} 8 \varepsilon-$
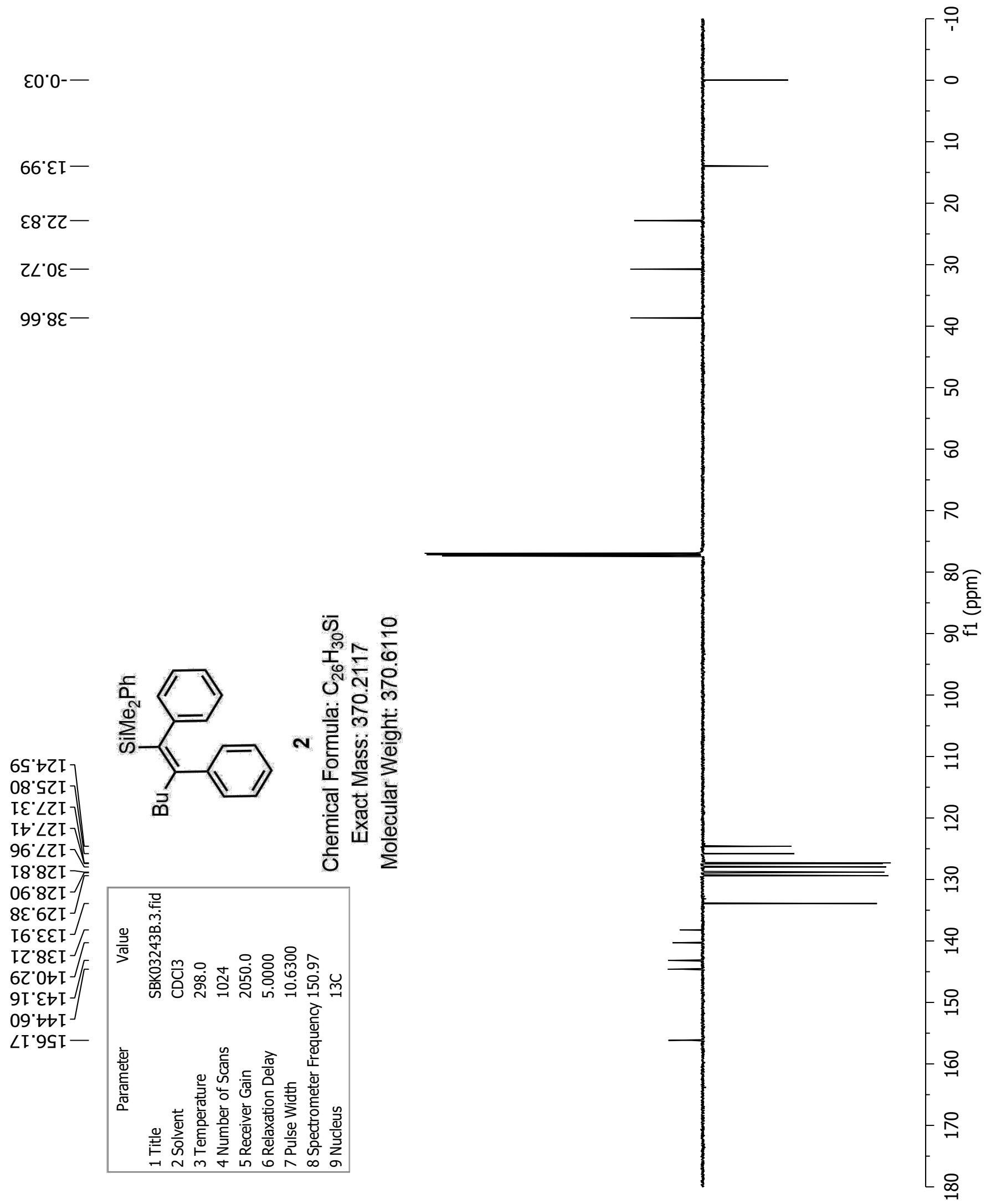


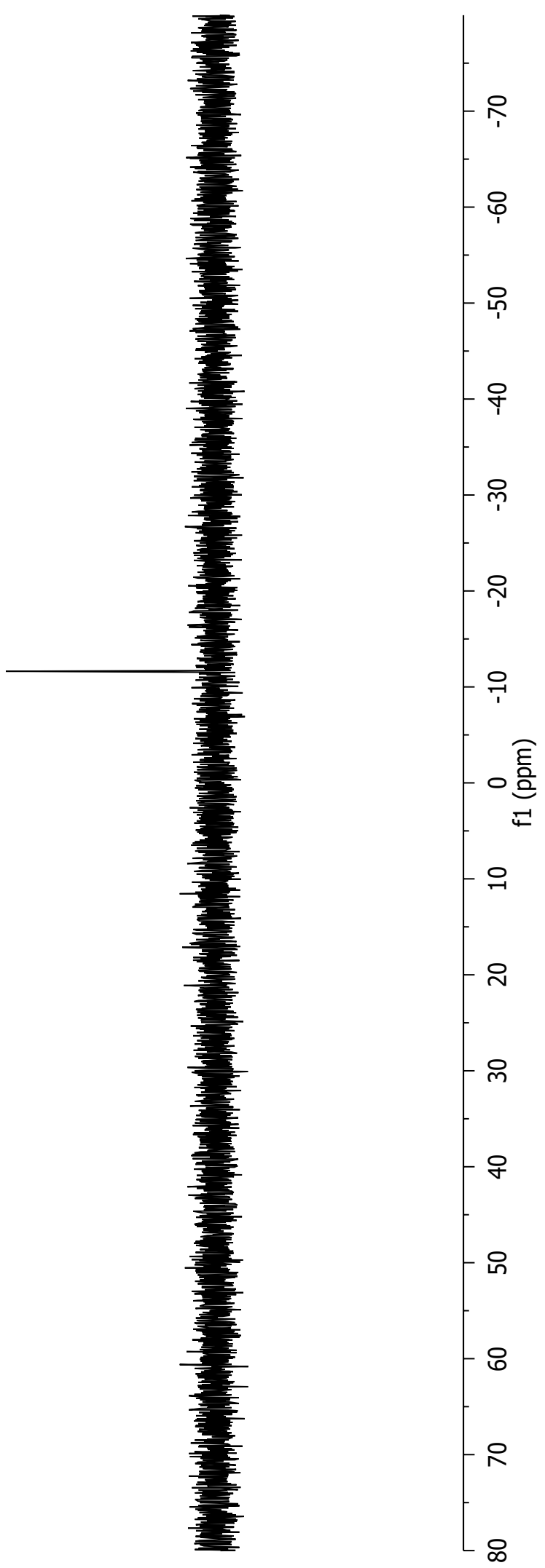


SI'0--

$\varepsilon 9^{\circ} 0$

†9 0

$59^{\circ} 0^{\circ}$

I0. I

20. I

ह0. I

$\angle 0^{\circ} \mathrm{I}$

$60 \%$

OI' [

II'Z

2I'Z

हI'乙

$08^{\circ} \varepsilon>$

โร $8^{\prime} \mathcal{}$
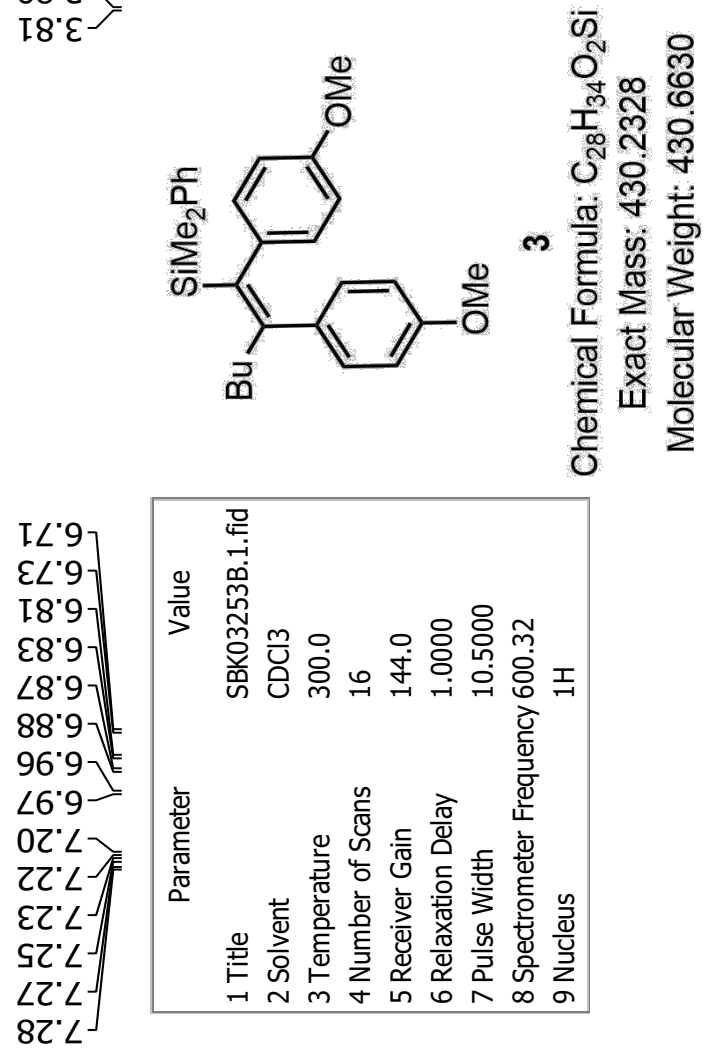

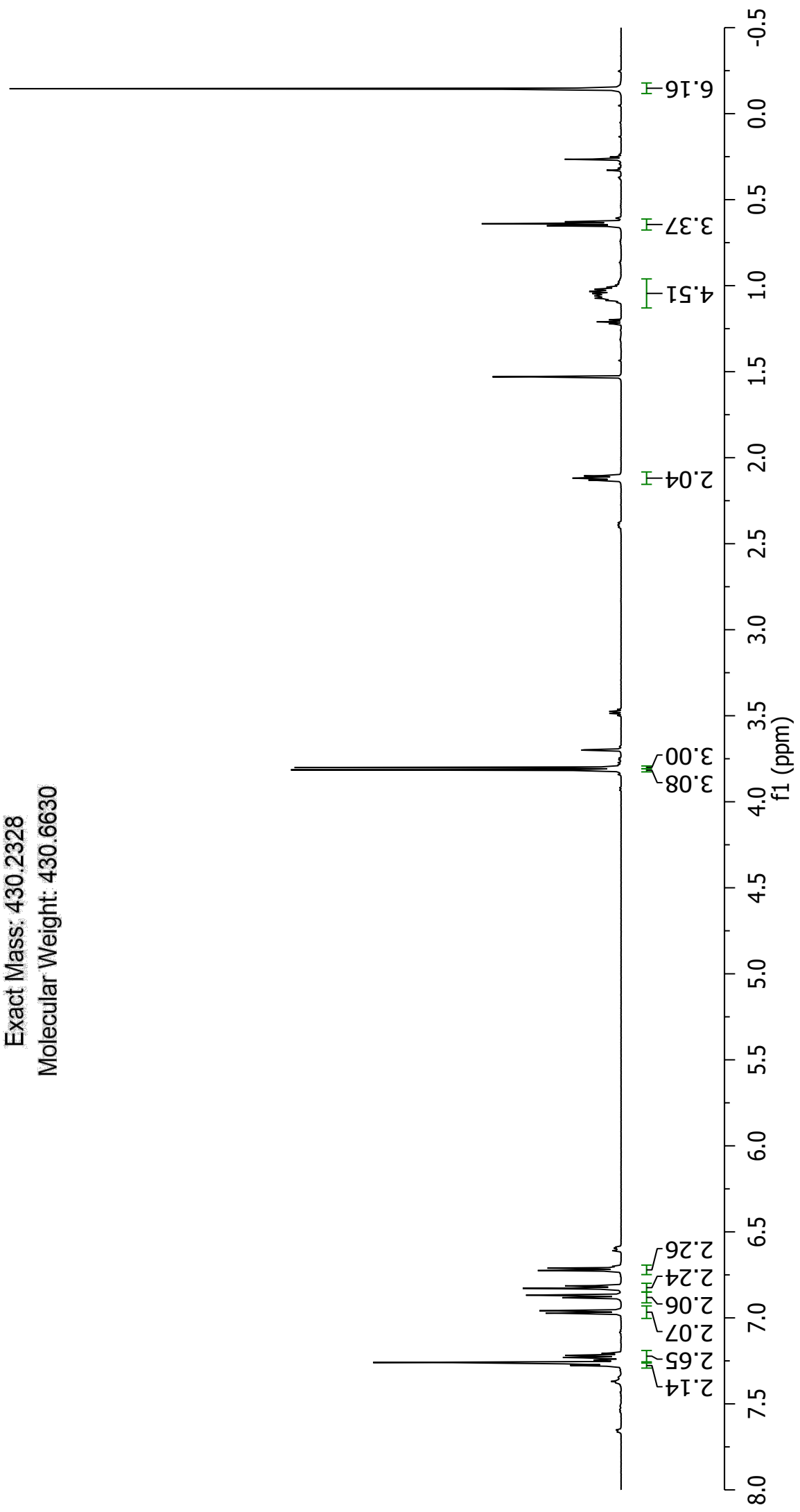


โ8.0--

$00 \div 5$

ย9'て૫-

St"0ع-

$60^{\circ} \angle \varepsilon-$

$\begin{aligned} & \downarrow \varepsilon \cdot s \\ & 9 \varepsilon \cdot s\end{aligned}>$

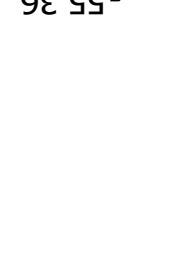

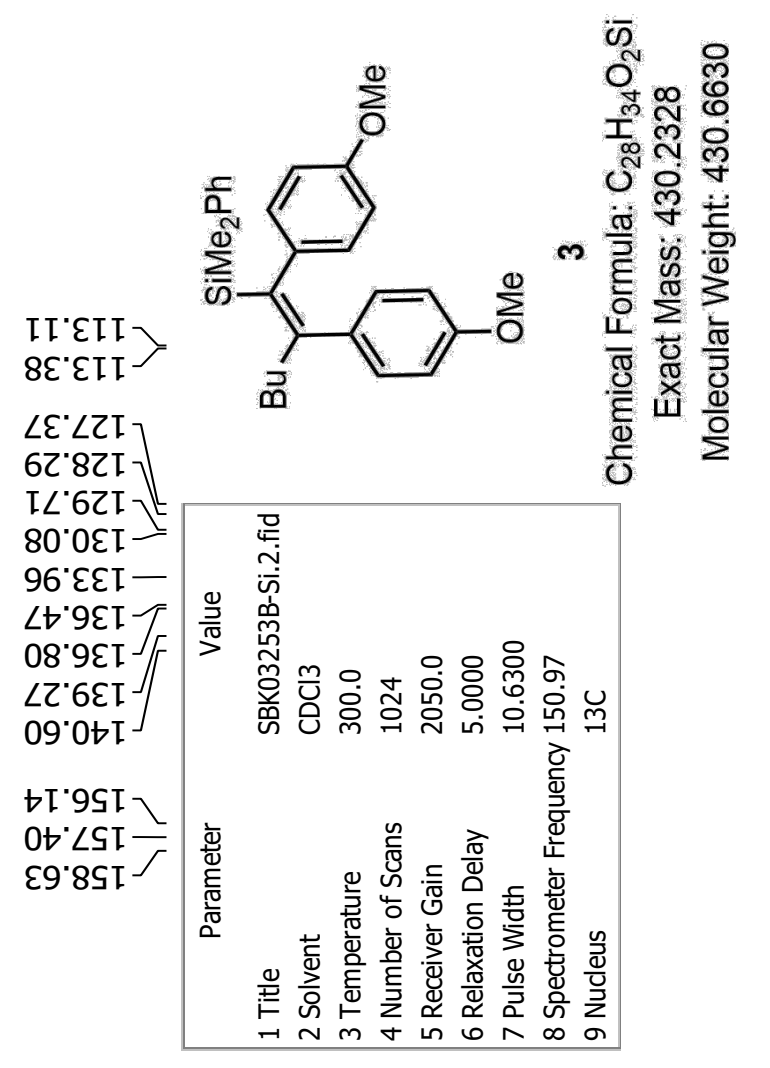

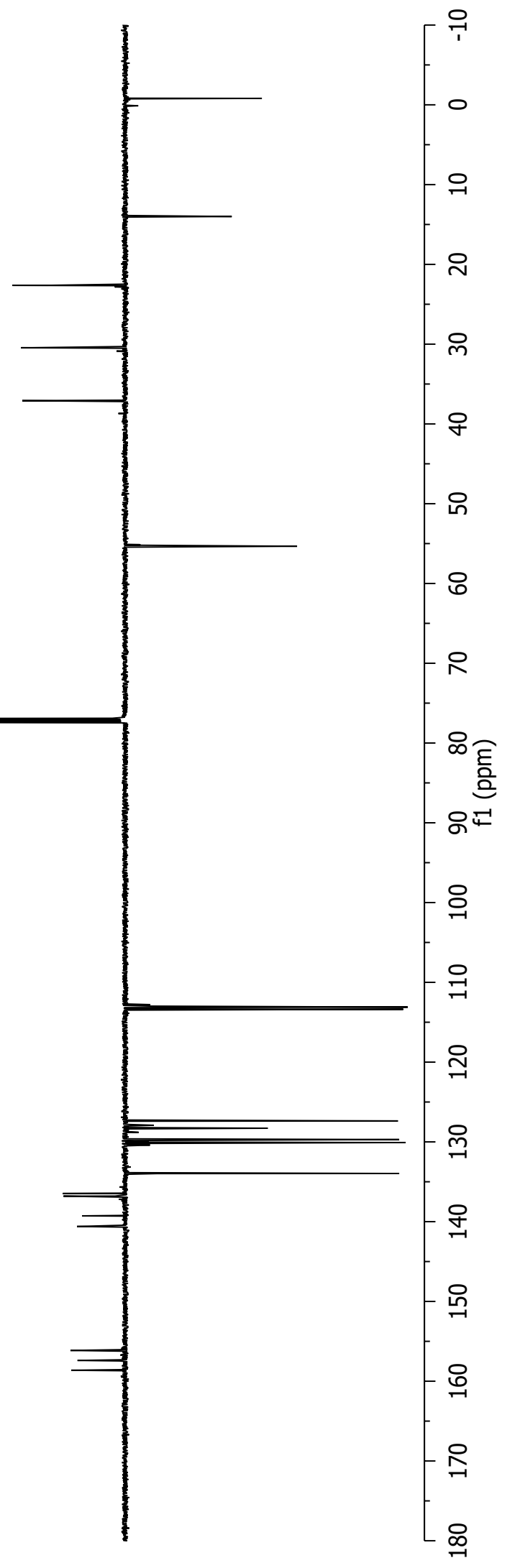



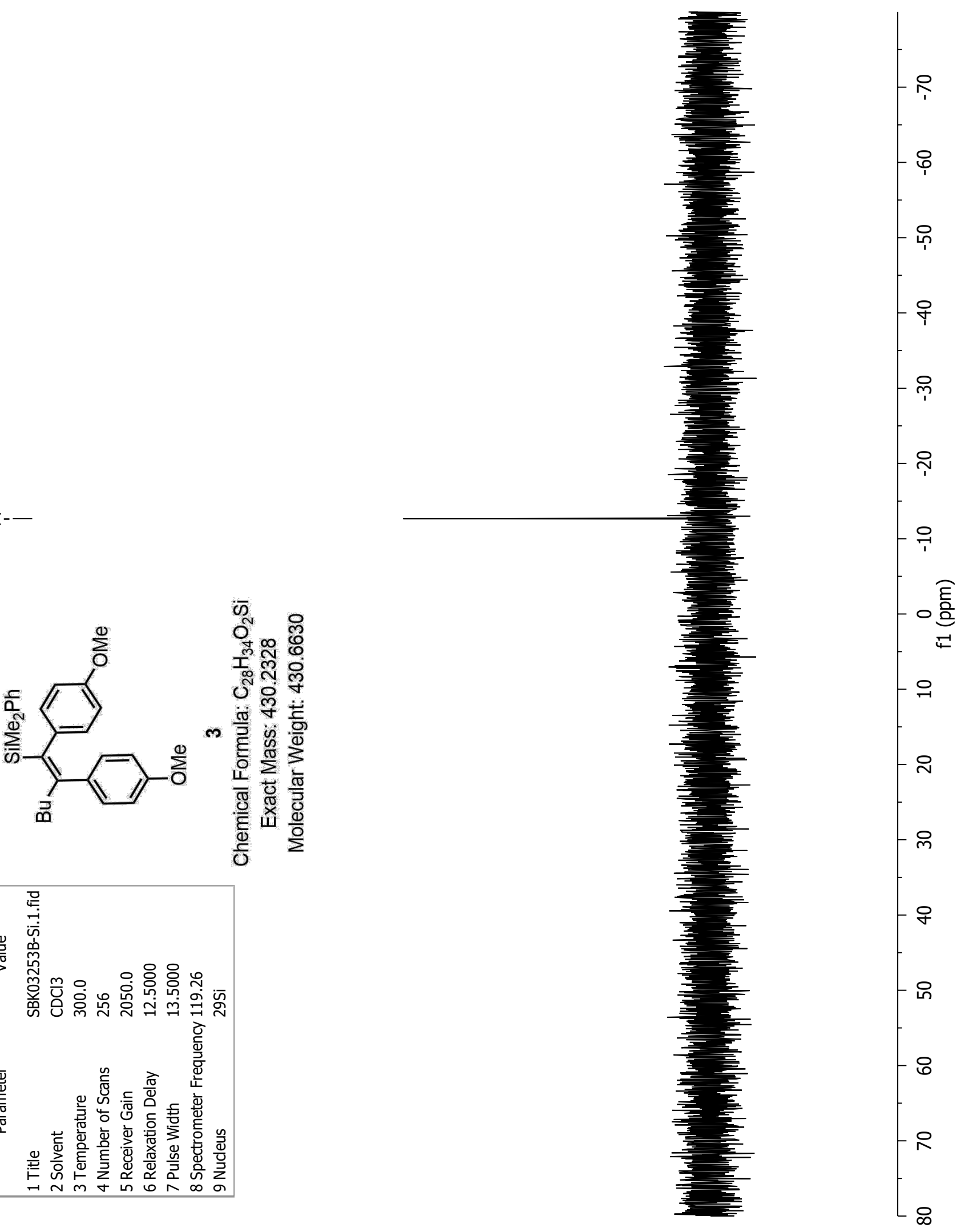
$8 \varepsilon^{\circ} 0$

$\varepsilon L^{\circ} 0$ $\downarrow \angle{ }^{\prime} 0$

$S \angle{ }^{\circ}{ }^{\circ}$

$80^{\circ} \mathrm{I}$

$80^{\circ} \mathrm{T}$

60.5

[S'Z]

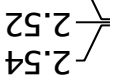

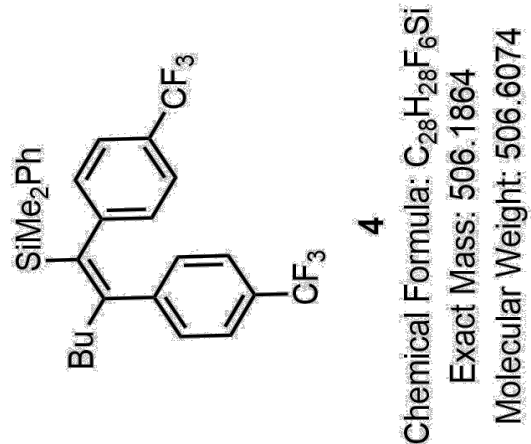

$\angle 6^{\circ} 9$

$86^{\circ} 9$
$\angle 0^{\circ} \angle$

$80^{\circ} \mathrm{L}$

$8 \varepsilon^{\circ} \angle$

$6 \varepsilon^{\prime} \angle$

$0 t^{\circ} \angle$

It $\angle$

$\left.8 t^{\circ} \angle\right]$

$8 t^{\circ} \angle$

$\left.\begin{array}{l}66^{\circ} \angle \\ T \angle L\end{array}\right]$ $Z \angle \angle L$ $2 \angle L Y$ $\varepsilon L L^{\prime} L$

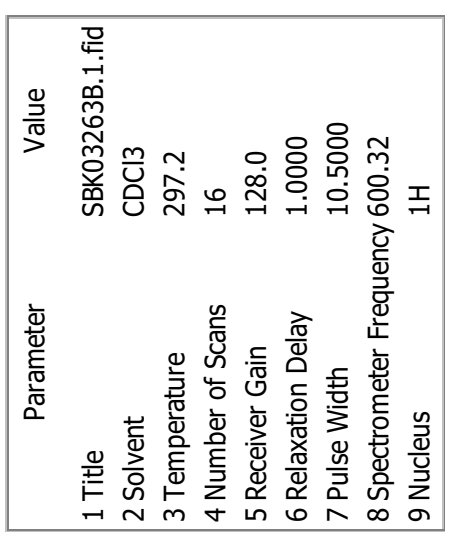

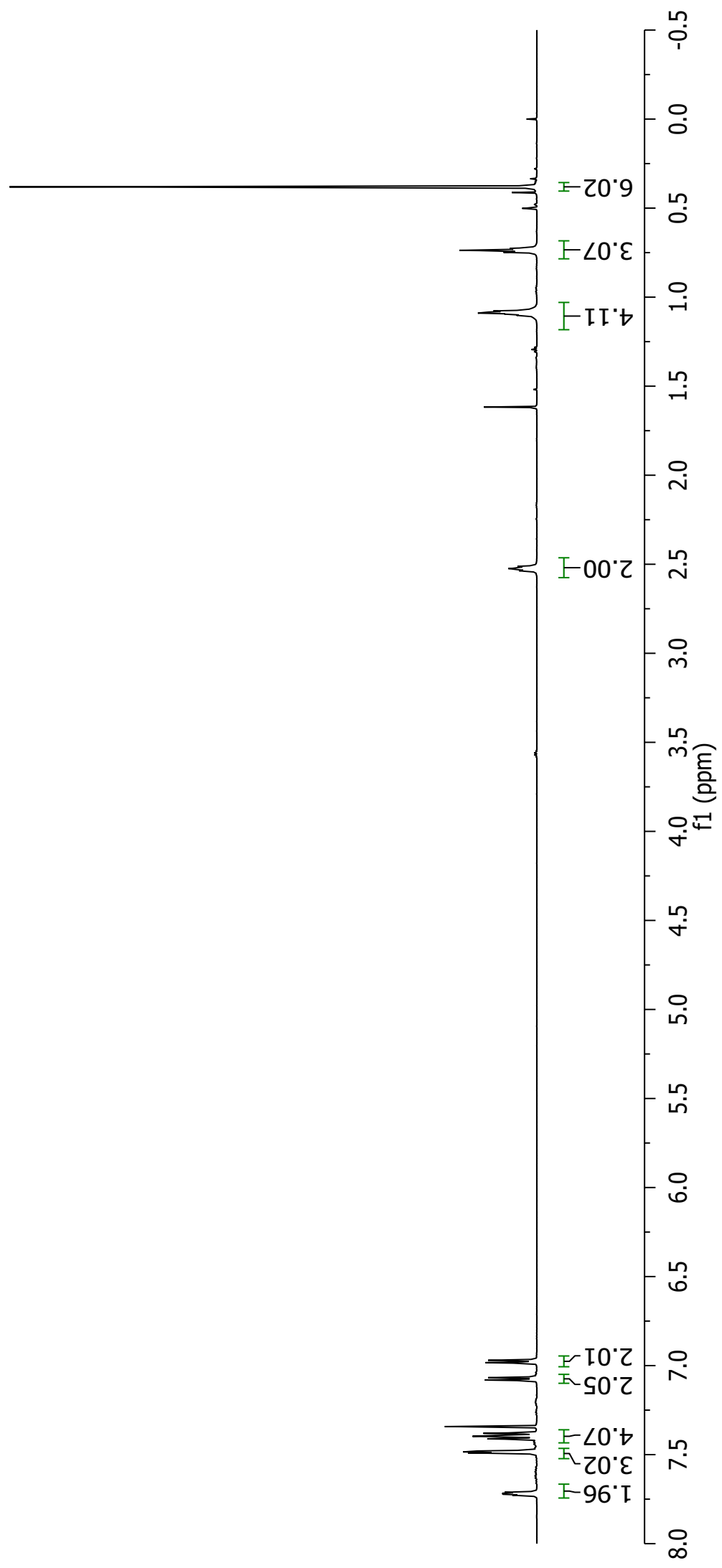




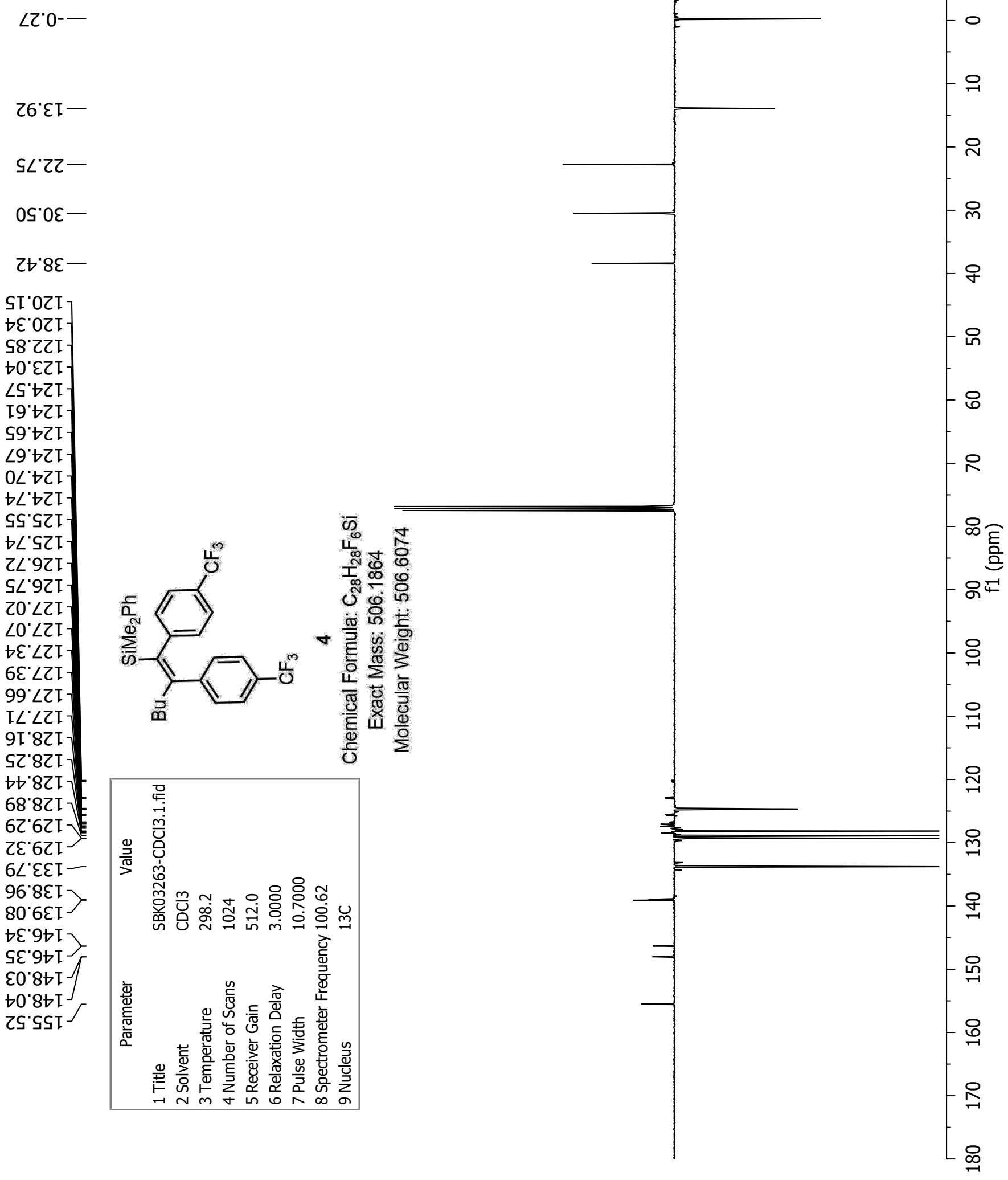



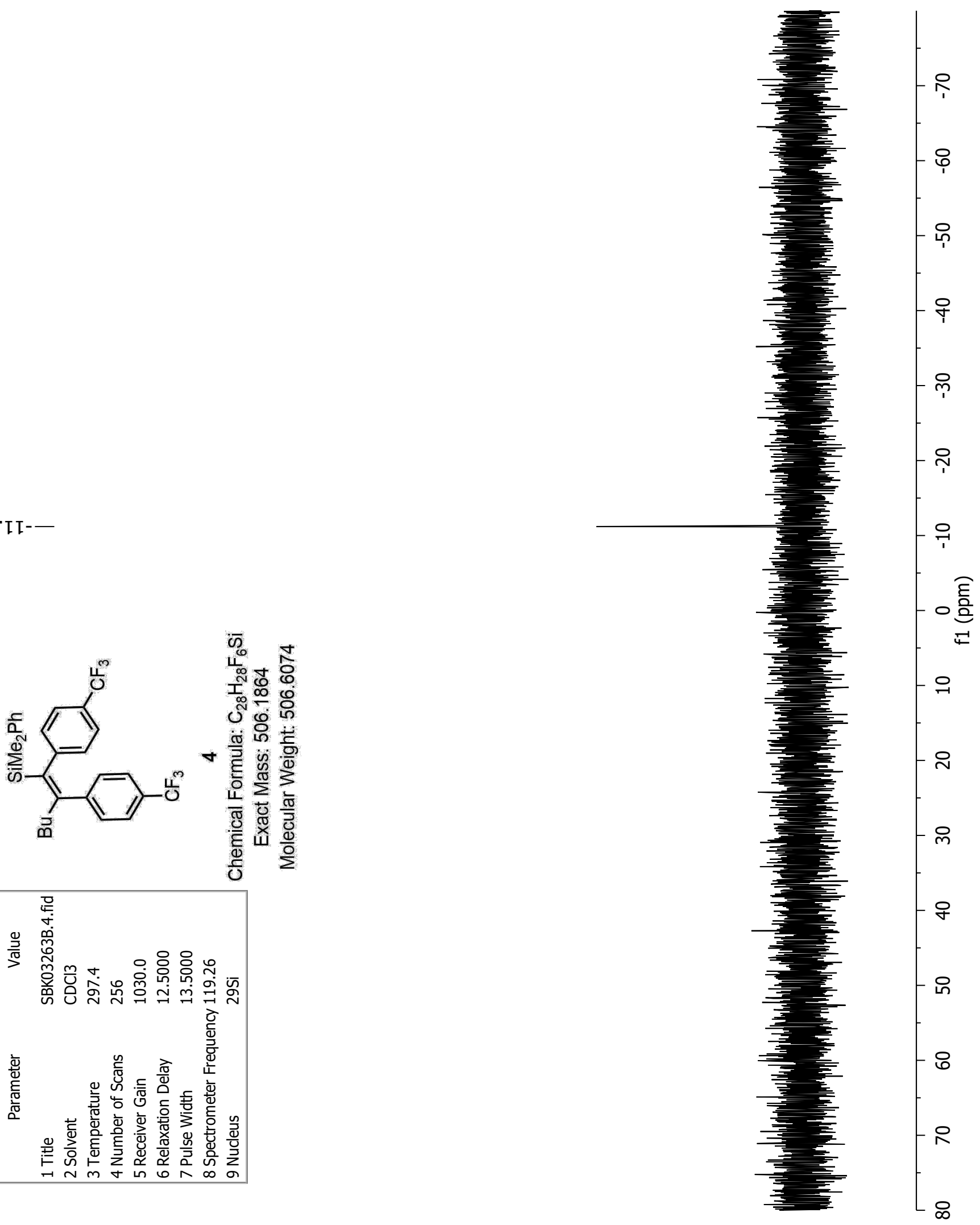


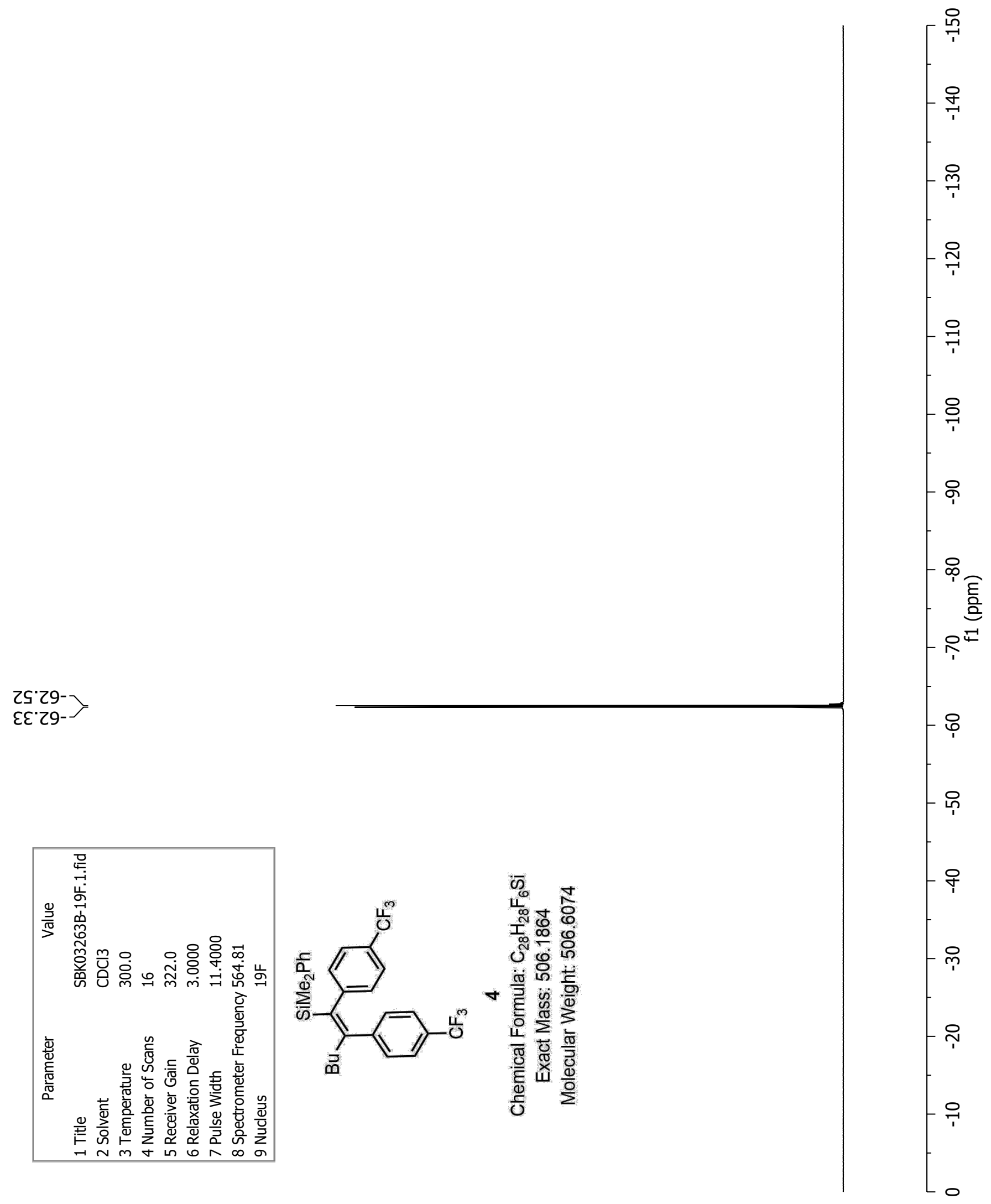


$82^{\circ} 0$

เ9.0

59.0

$99^{\circ} 0$

$86^{\circ} 0$

$66^{\circ} 0$

$00^{\circ} \mathrm{I}$

[0. I

$8 \varepsilon^{\prime}$ 乙

O৮' $\frac{\square}{T}$

I七て
0<' 9

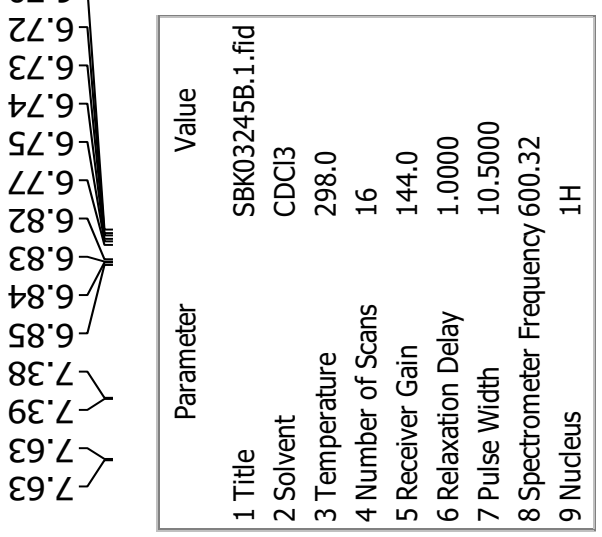

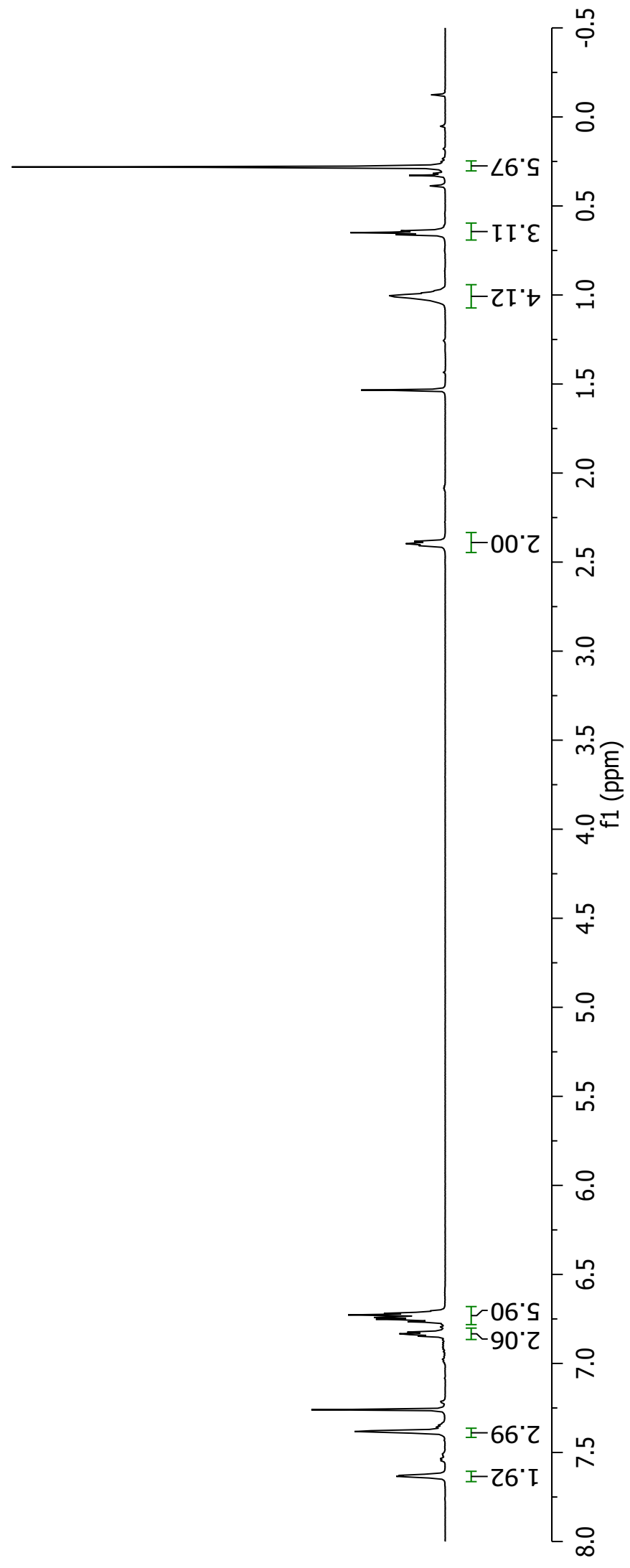



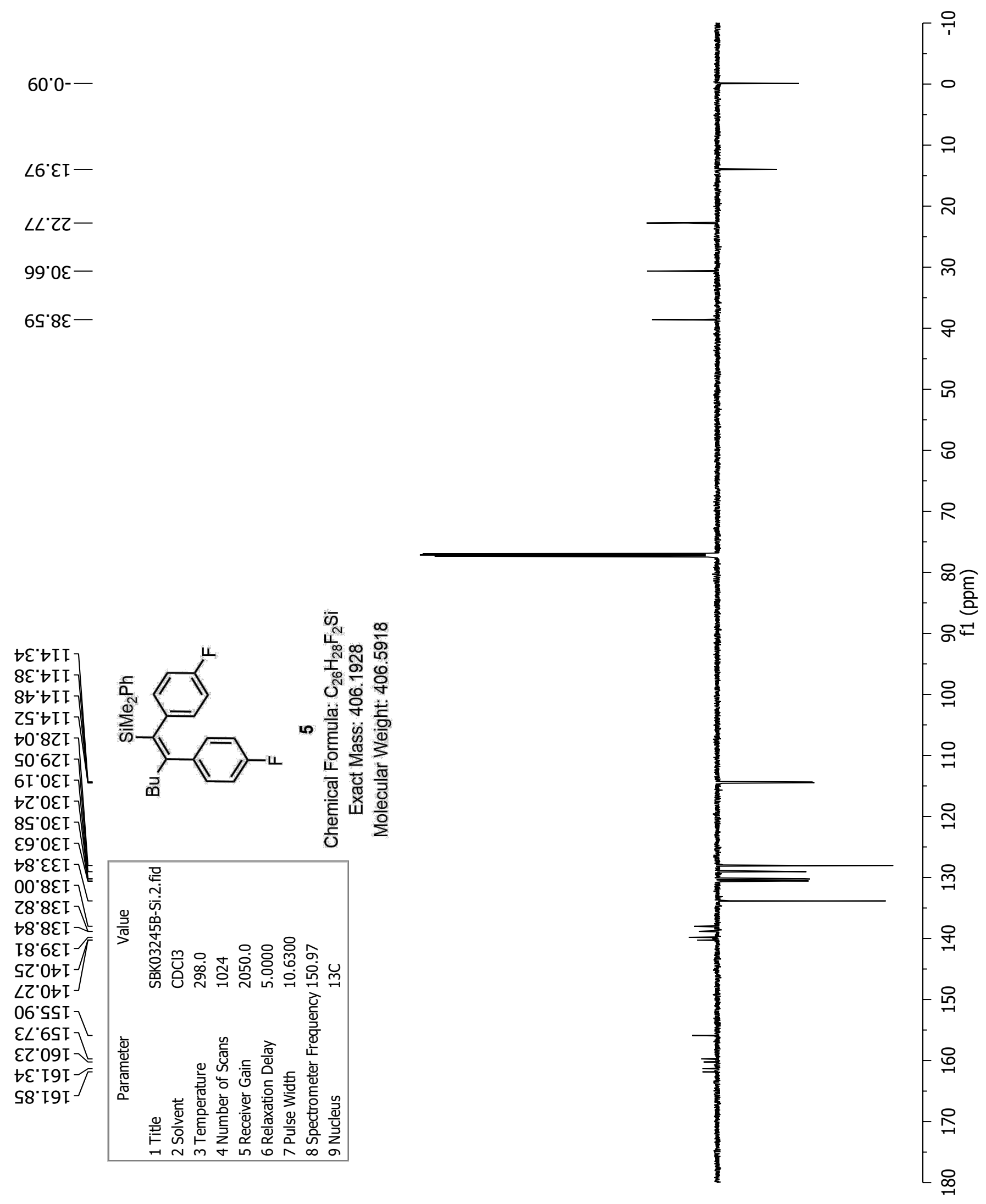


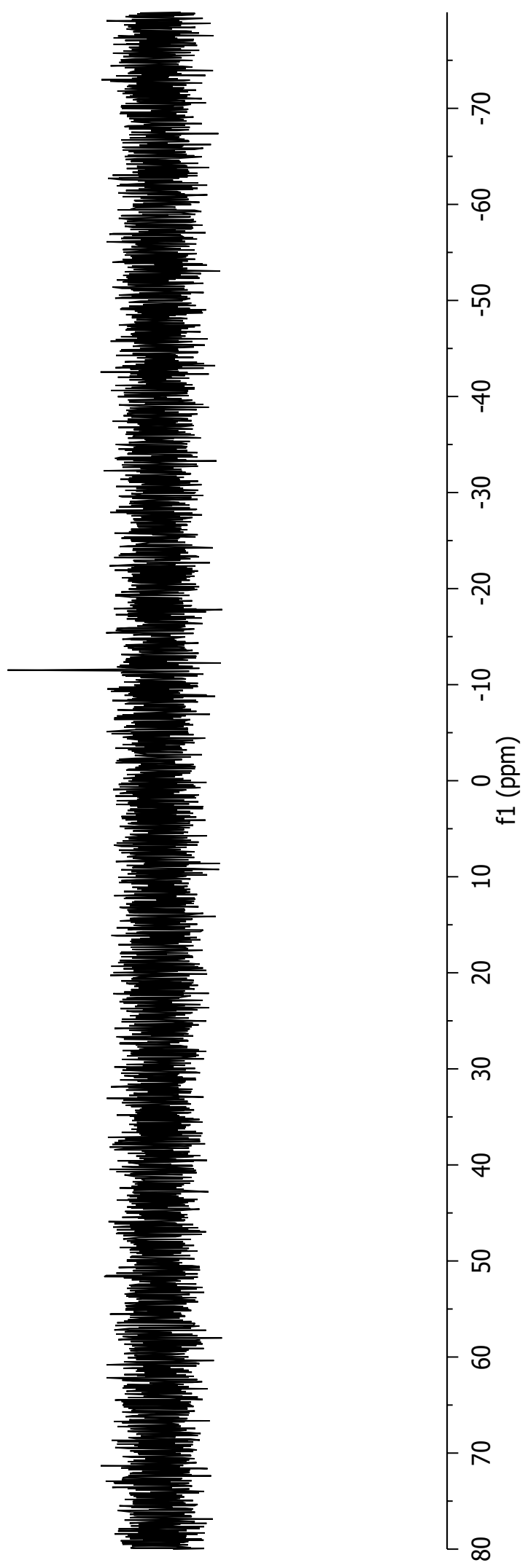



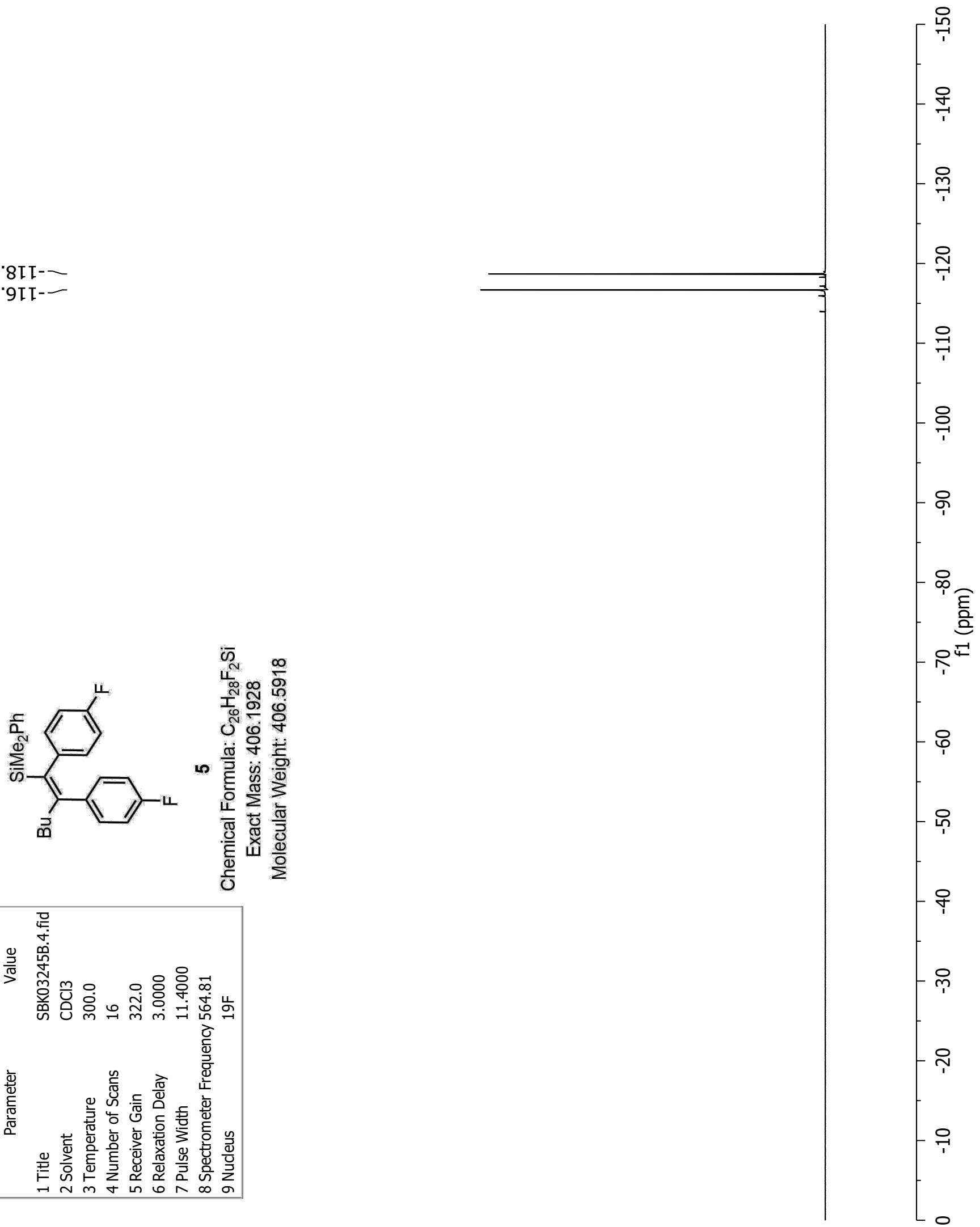
$\angle 20^{\circ}-$

乙9.0

$\varepsilon 9^{\circ} 0$

t9.0-

เ9.0

$99^{\circ} 0$

$86^{\circ} 0$

$66^{\circ} 0^{-}$

$00 . \mathrm{I}$

[0. I

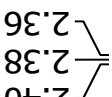

$0 t \mathrm{Z}$

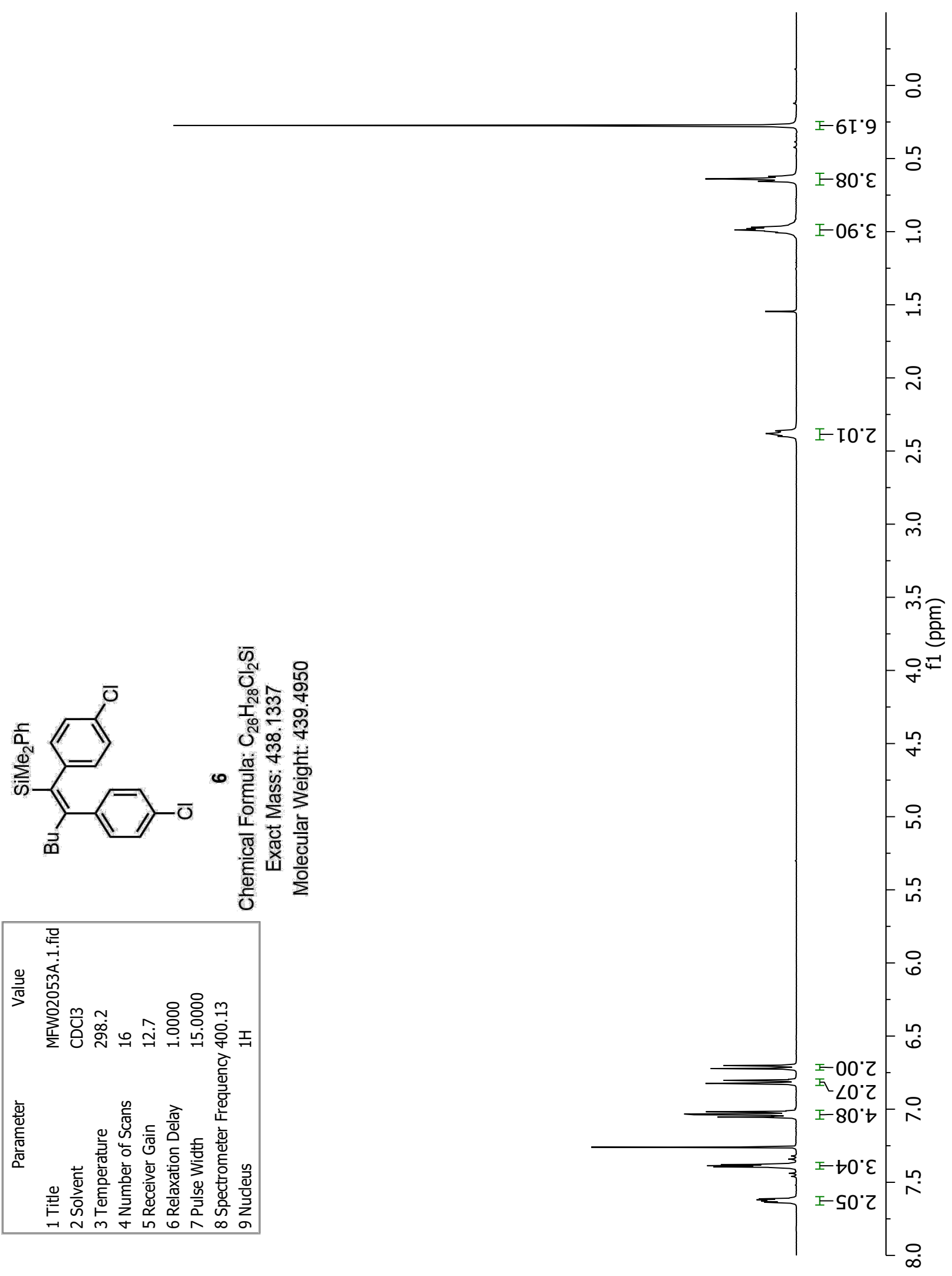


عI"0-—

$\angle 6^{\circ} \varepsilon I-$

$9 L 2 Z$

$6 S^{\circ} 0 \varepsilon-$

$\angle t^{\circ} 8 \varepsilon-$
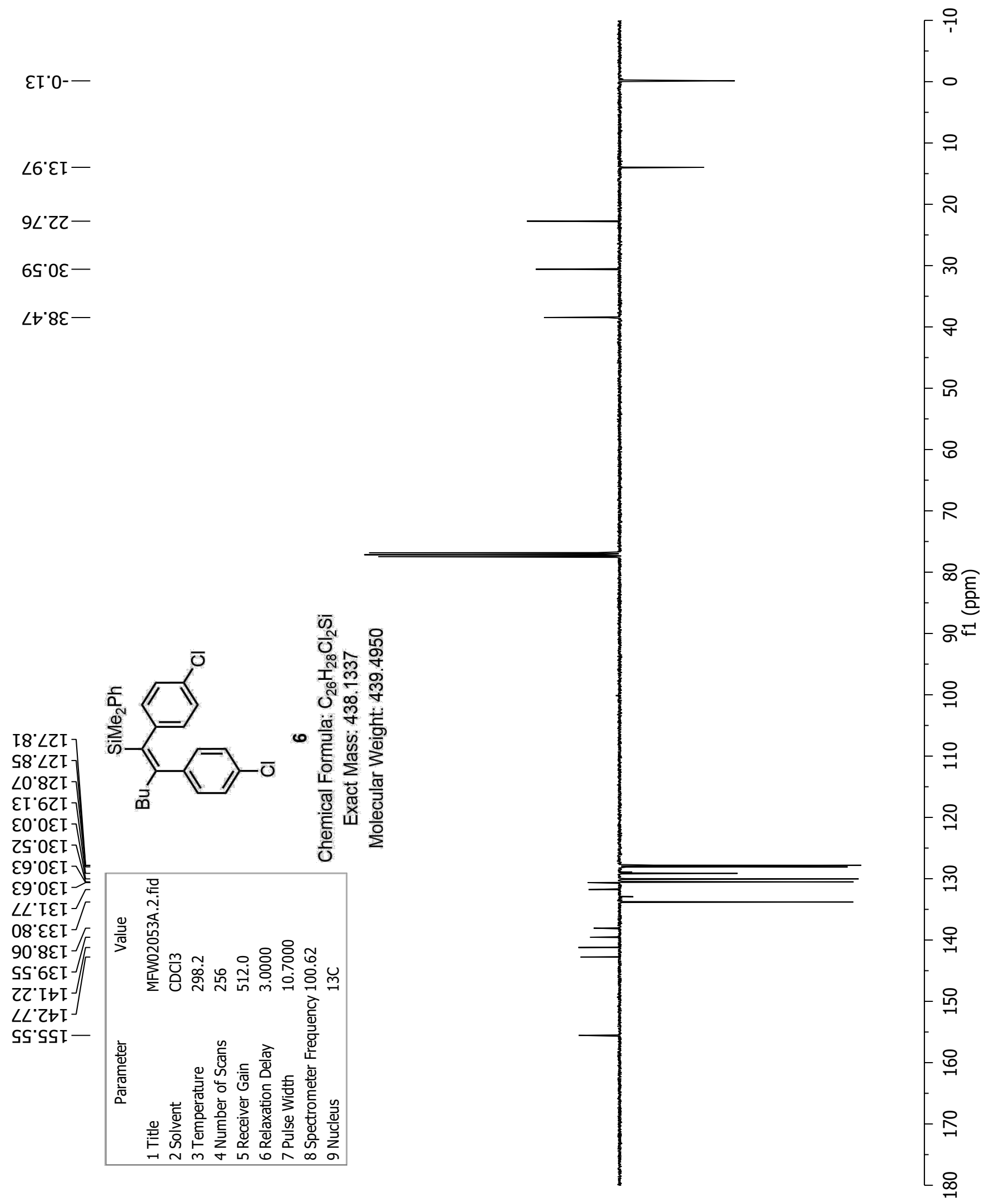


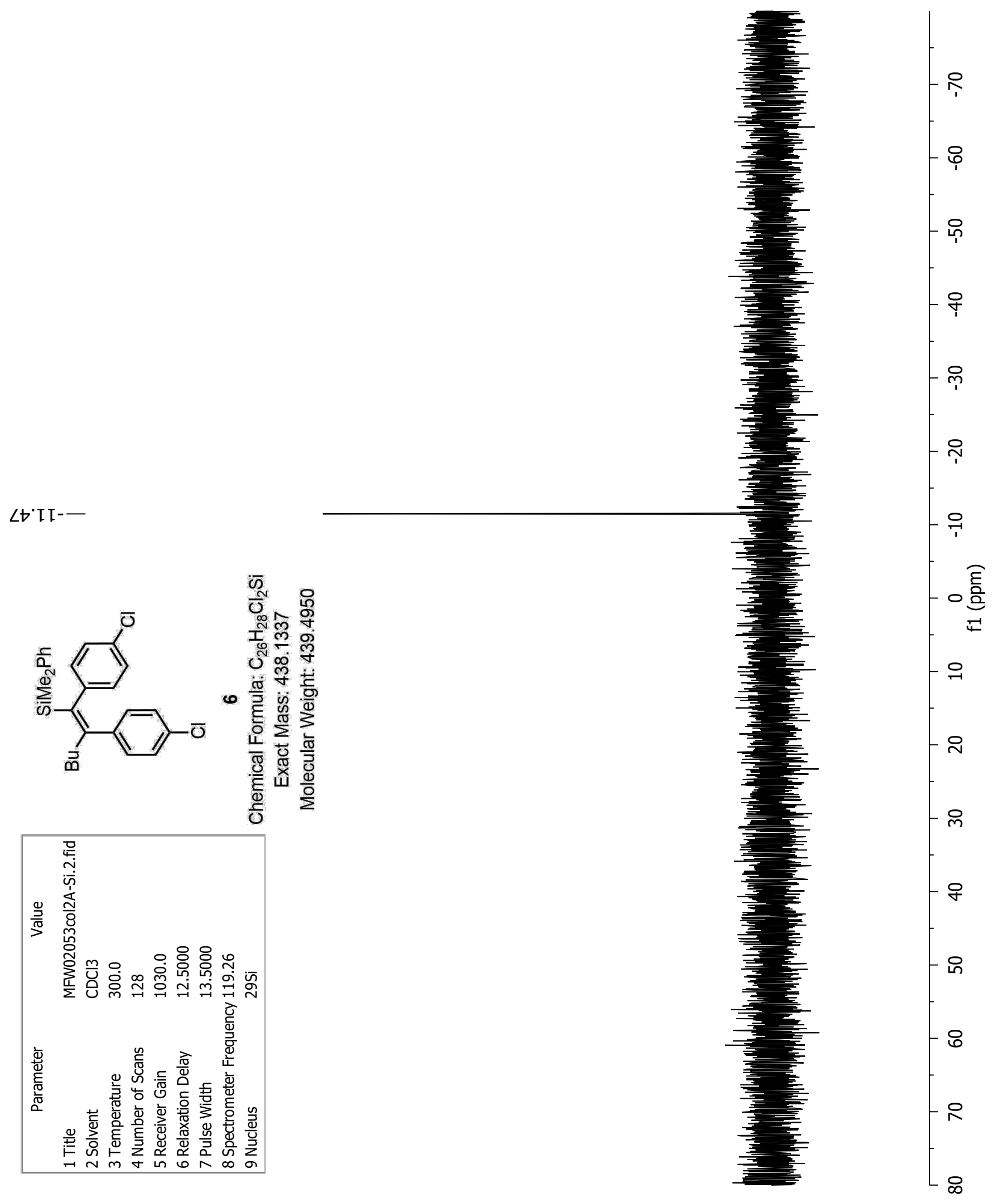


$62^{\circ} 0$ $\downarrow 9^{\circ} 0$ S9.0 $\angle 9^{\circ} 0$

$86^{\circ} 0$ $66^{\circ} 0$ J 00 ' I

$8 \varepsilon^{\prime} \mathrm{Z}$ $0 t: 2$ It 2
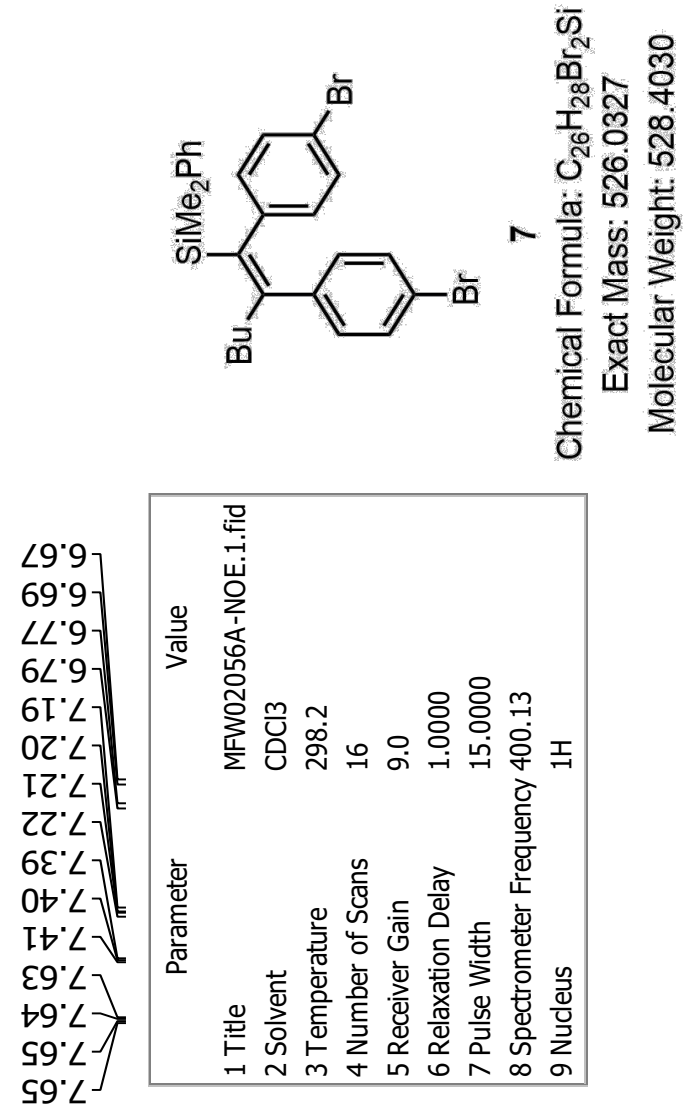

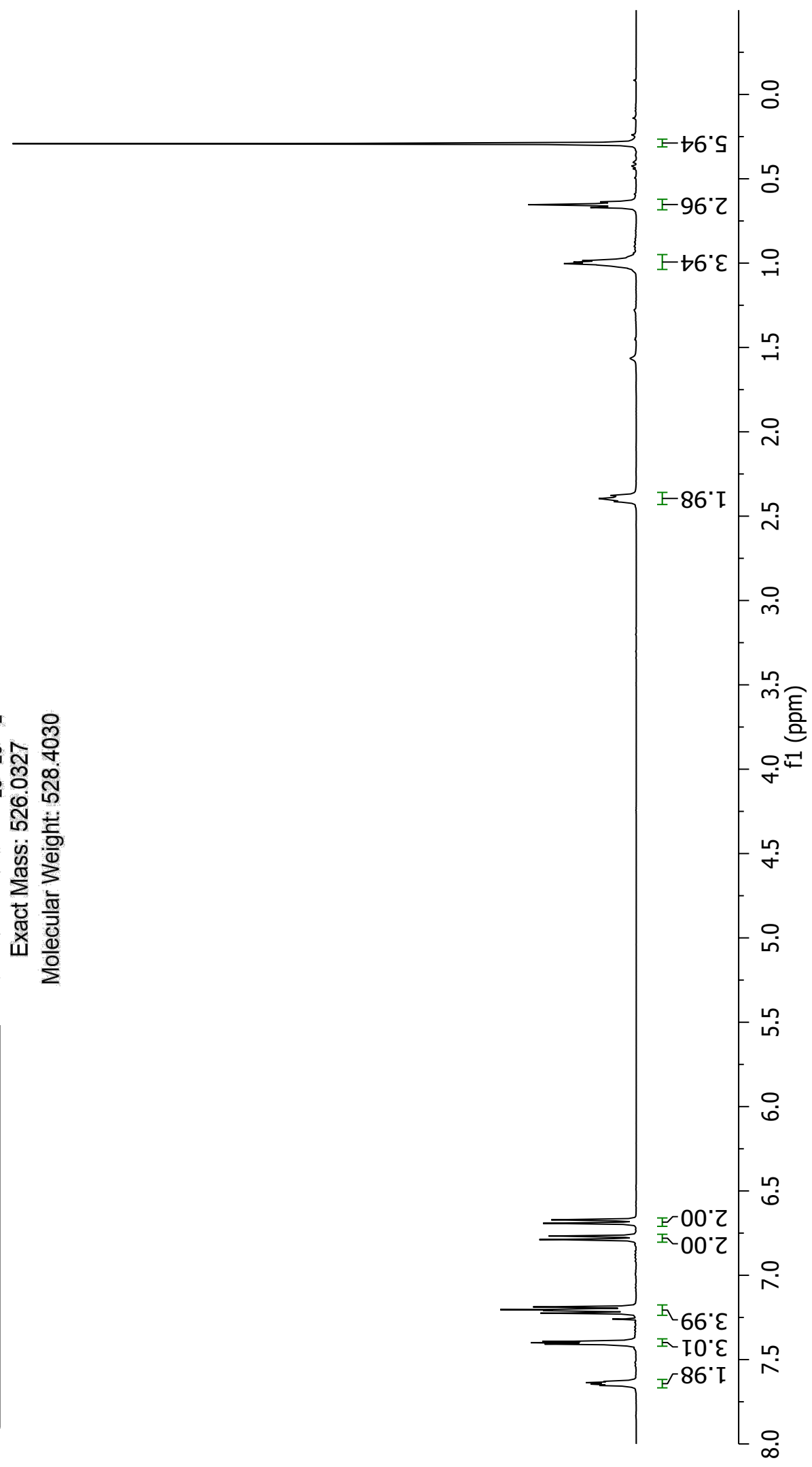




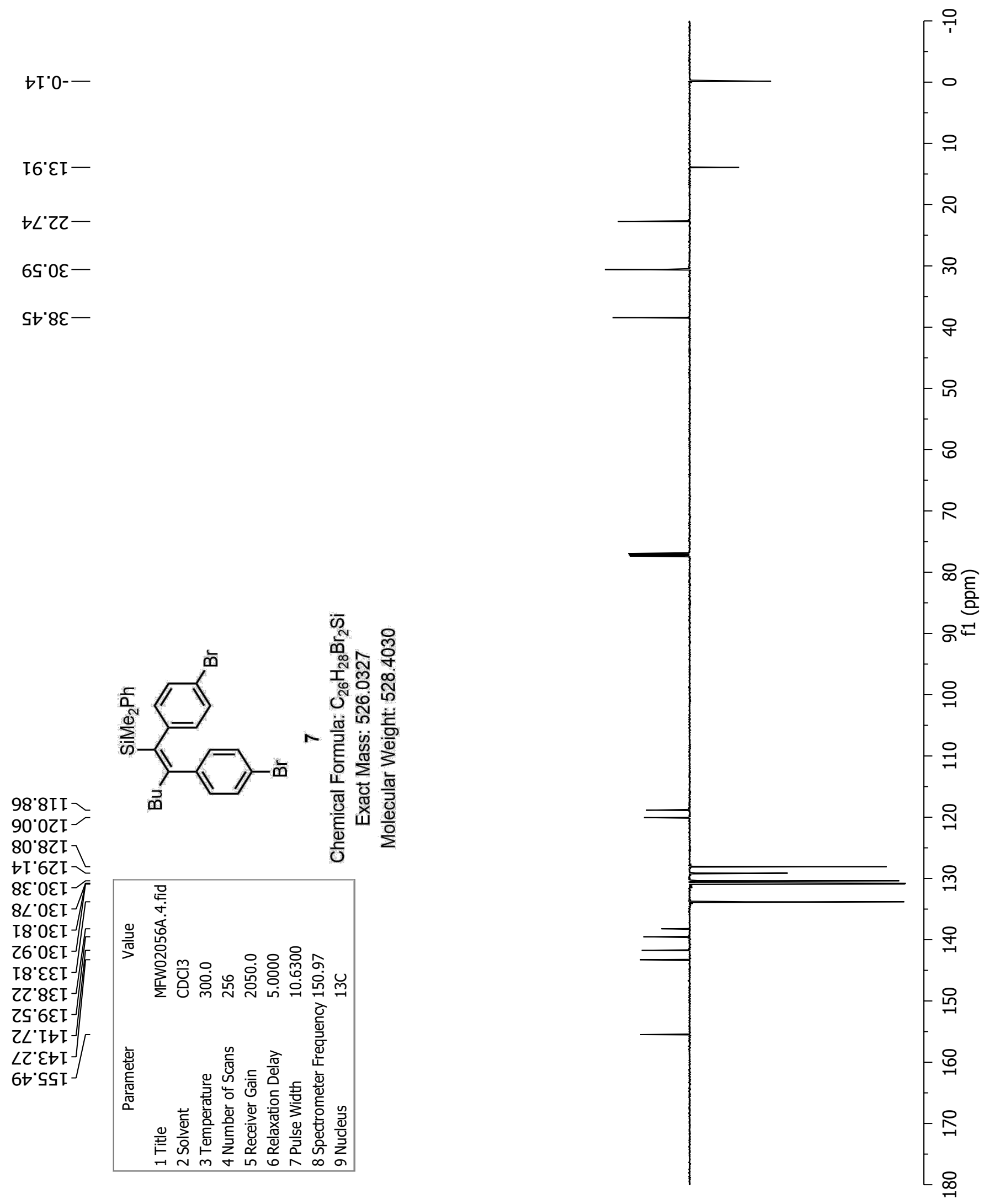


6t'II-

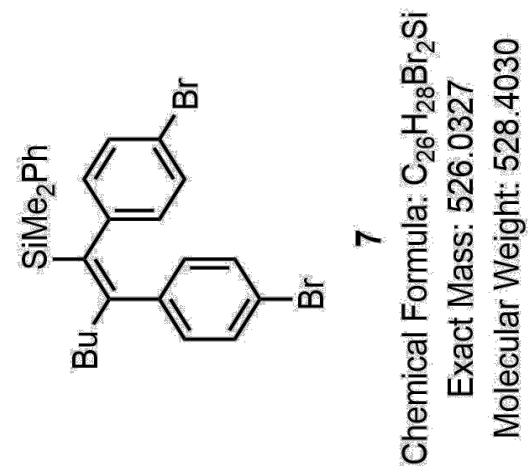

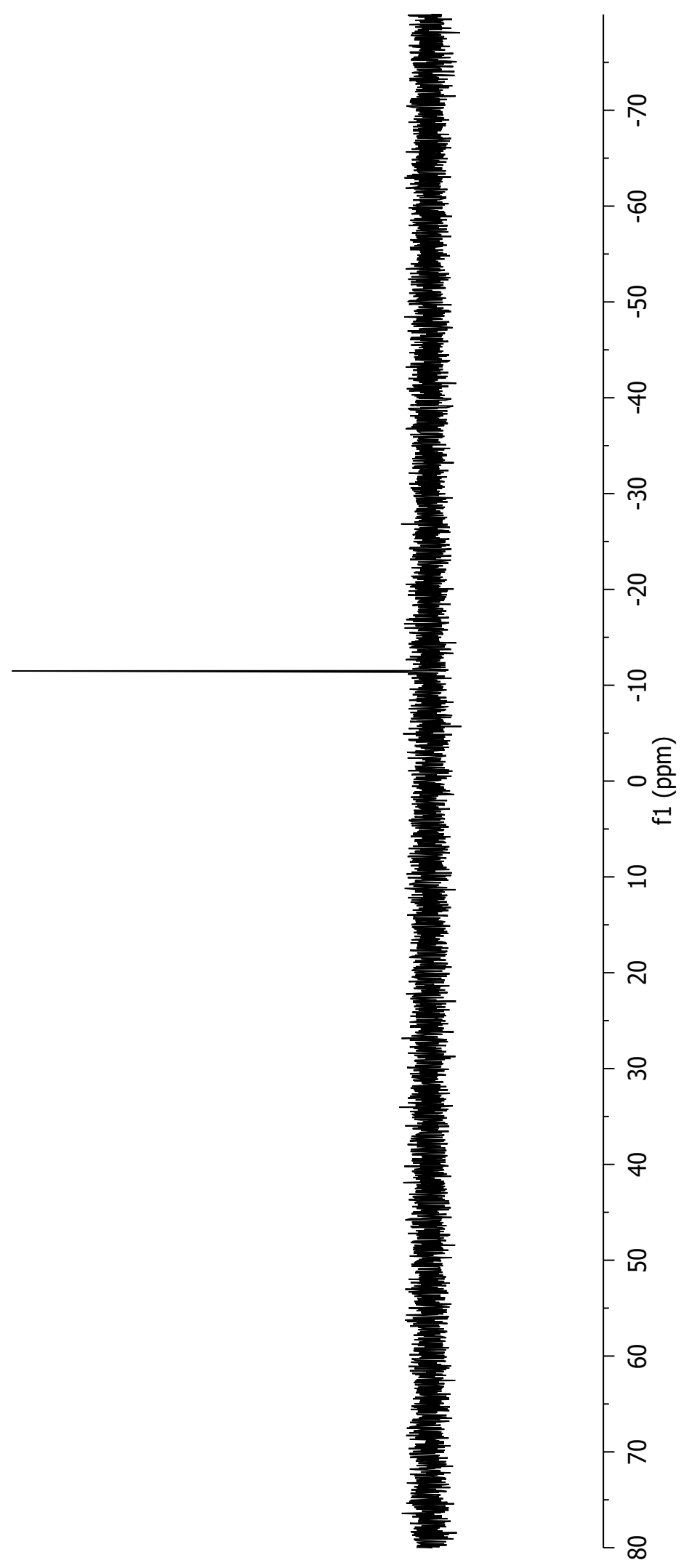


$\angle C^{\circ} 0-$

$\varepsilon 9^{\circ} 0$

ร9.0

99.0

$96^{\circ} 0$

$\angle 6^{\circ} 0$

00 . I

[0. I

20. I

E. I I -

SO I -

90. I-

$\angle 0^{\circ} \mathrm{I}$

$\left.\begin{array}{c}\angle I \cdot Z \\ 8 \varepsilon^{\prime} \cdot\end{array}\right]$

$0+2$

It 2

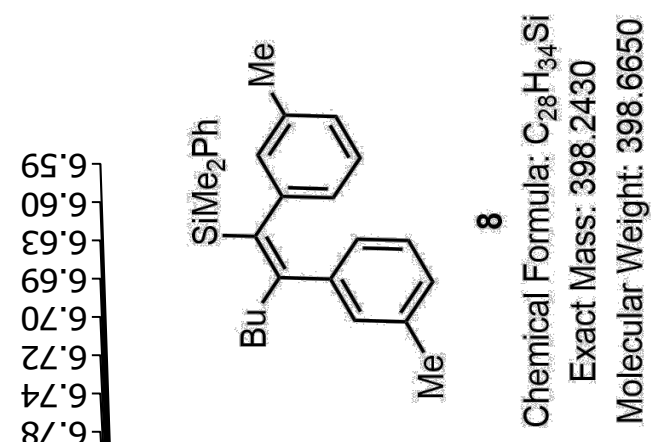

$8 \angle ' 9$

6L'9-

06.9

โ6.9

Z6.9

ع6.9-

$9 \varepsilon^{\prime} L$

$\left.8 \varepsilon^{\circ} \angle\right]$

$\left.8 \varepsilon^{\circ} \angle\right]$

$\left.0 t^{\circ} \angle\right]$

$0 t^{\circ} \angle \sqrt{ }$

$89^{\circ} \mathrm{L}$

$89^{\circ} \angle>$

$69^{\circ} \angle \mathcal{J}$

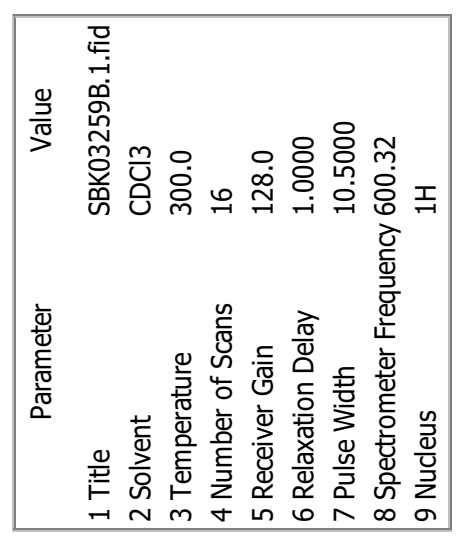

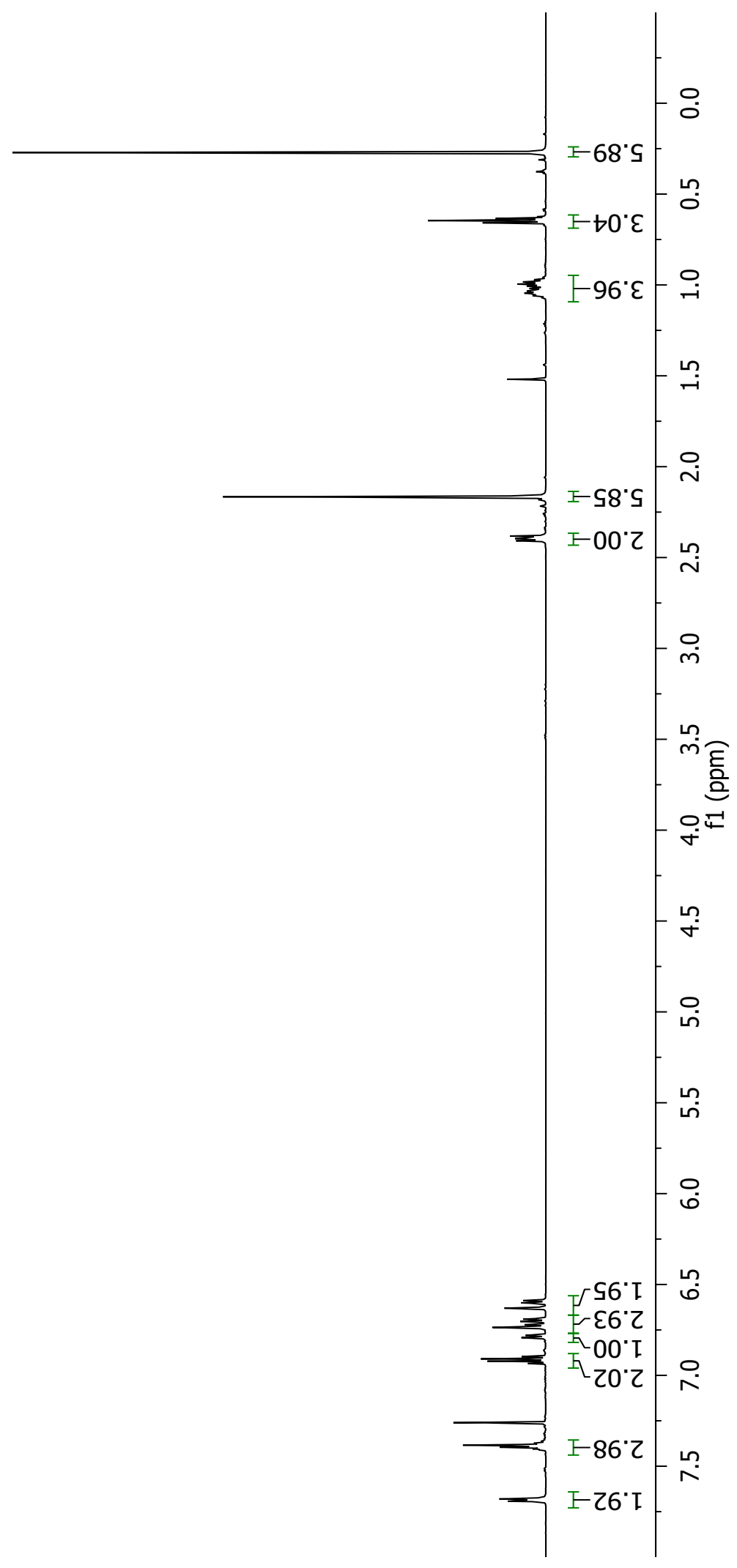




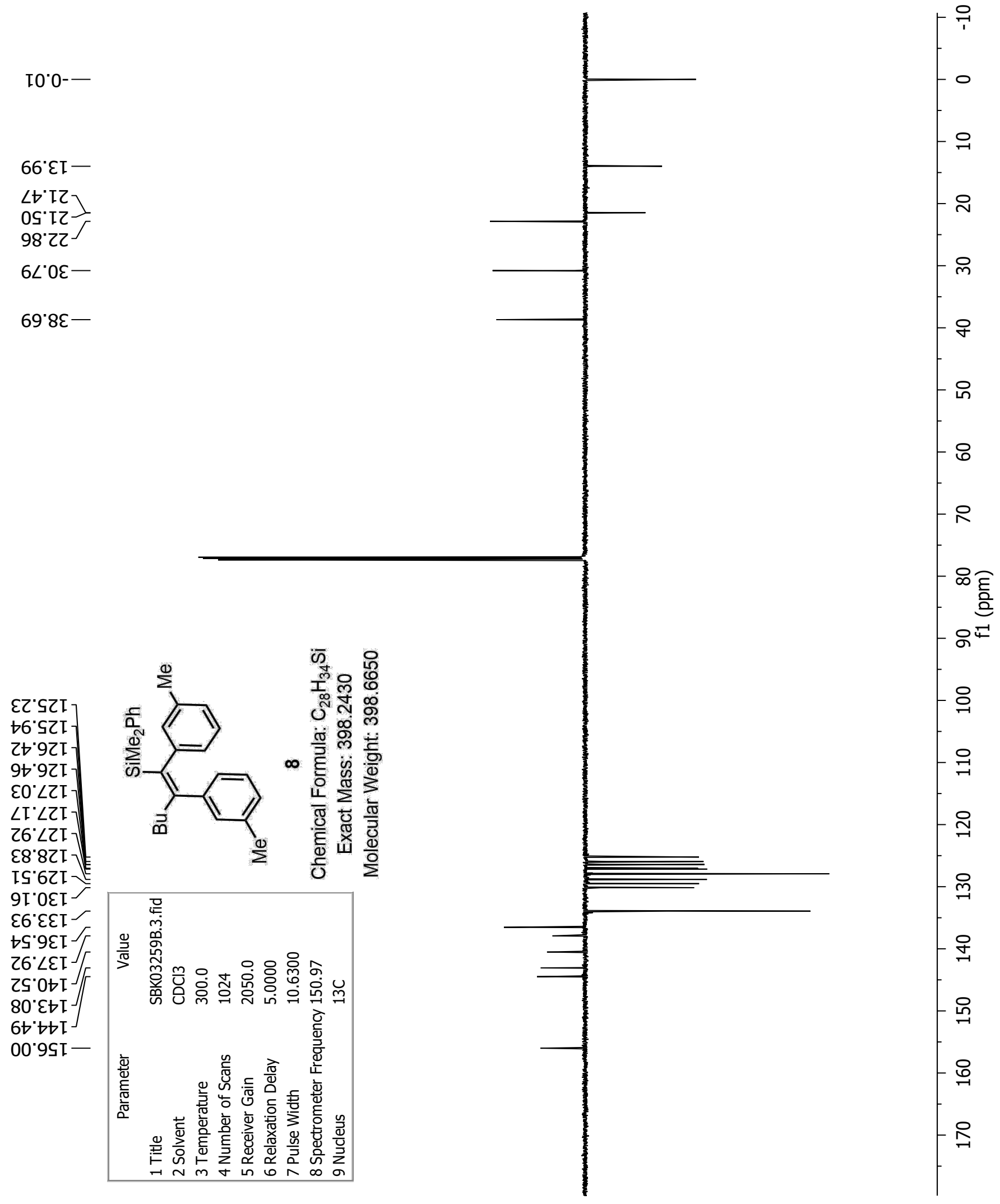



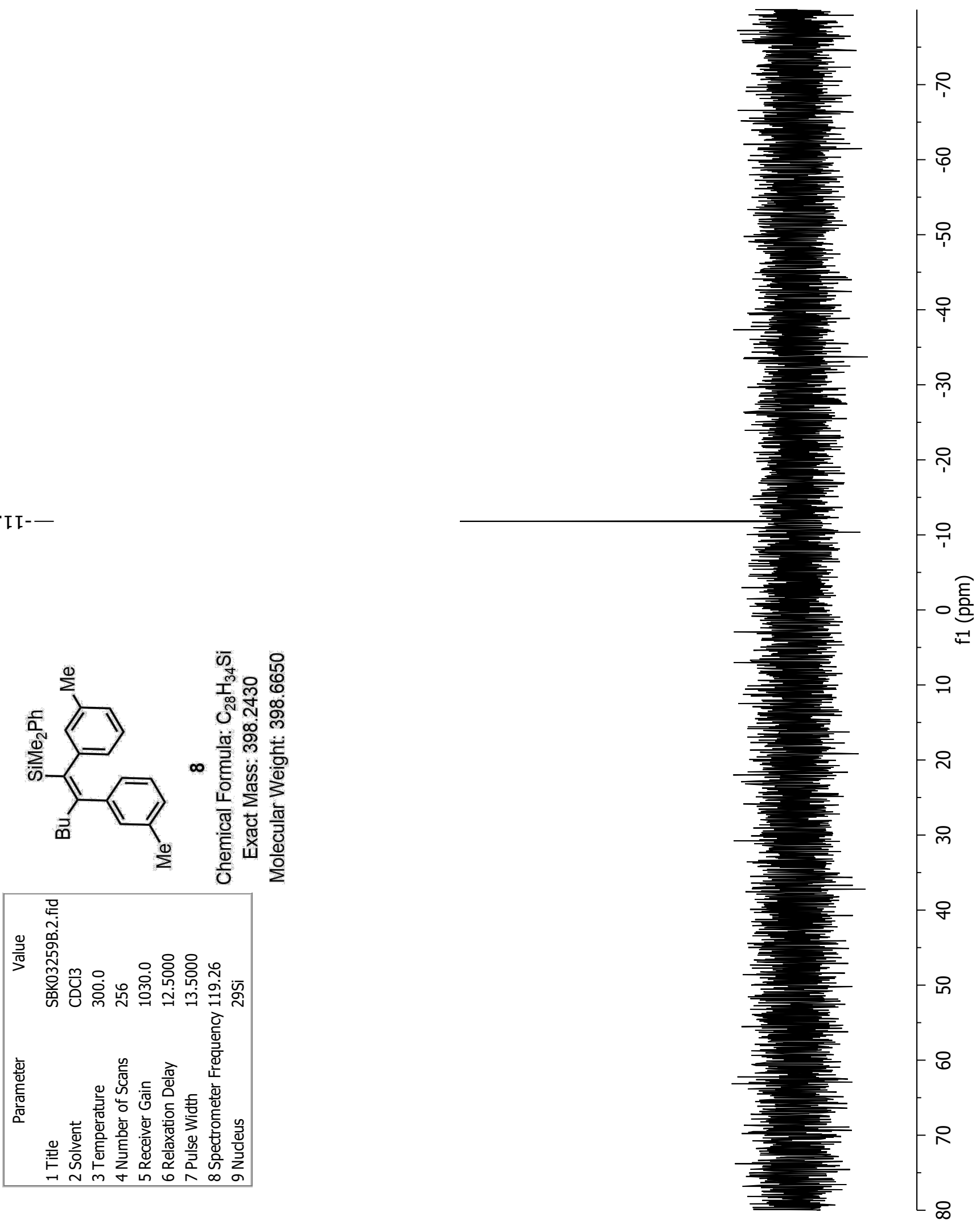


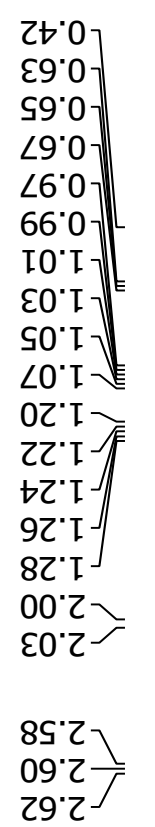

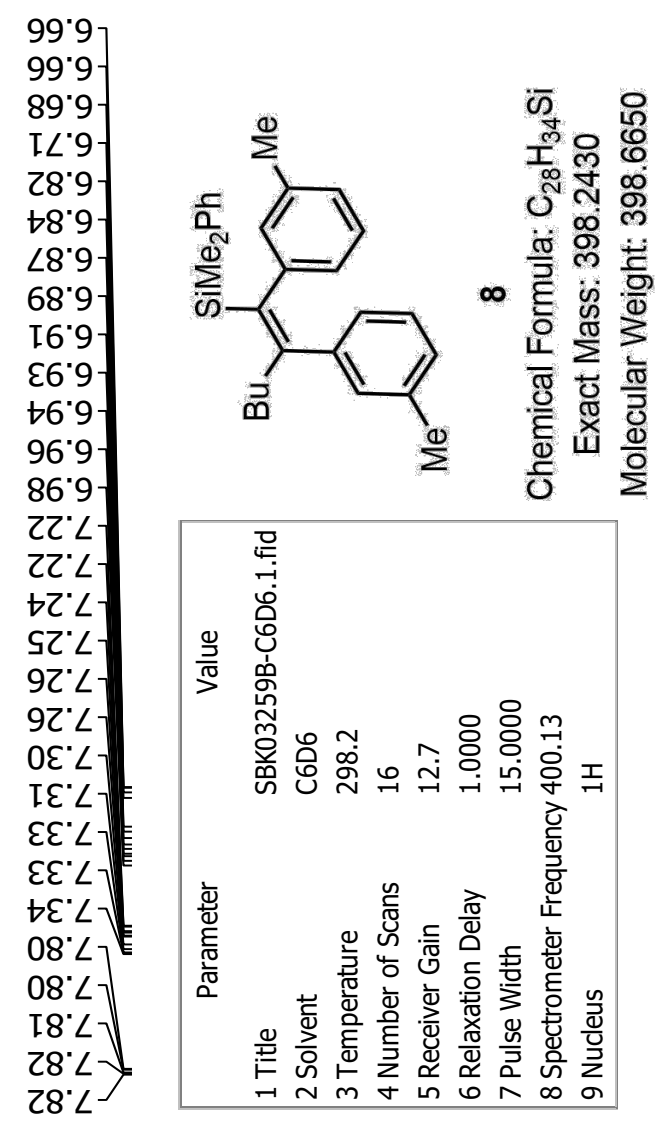

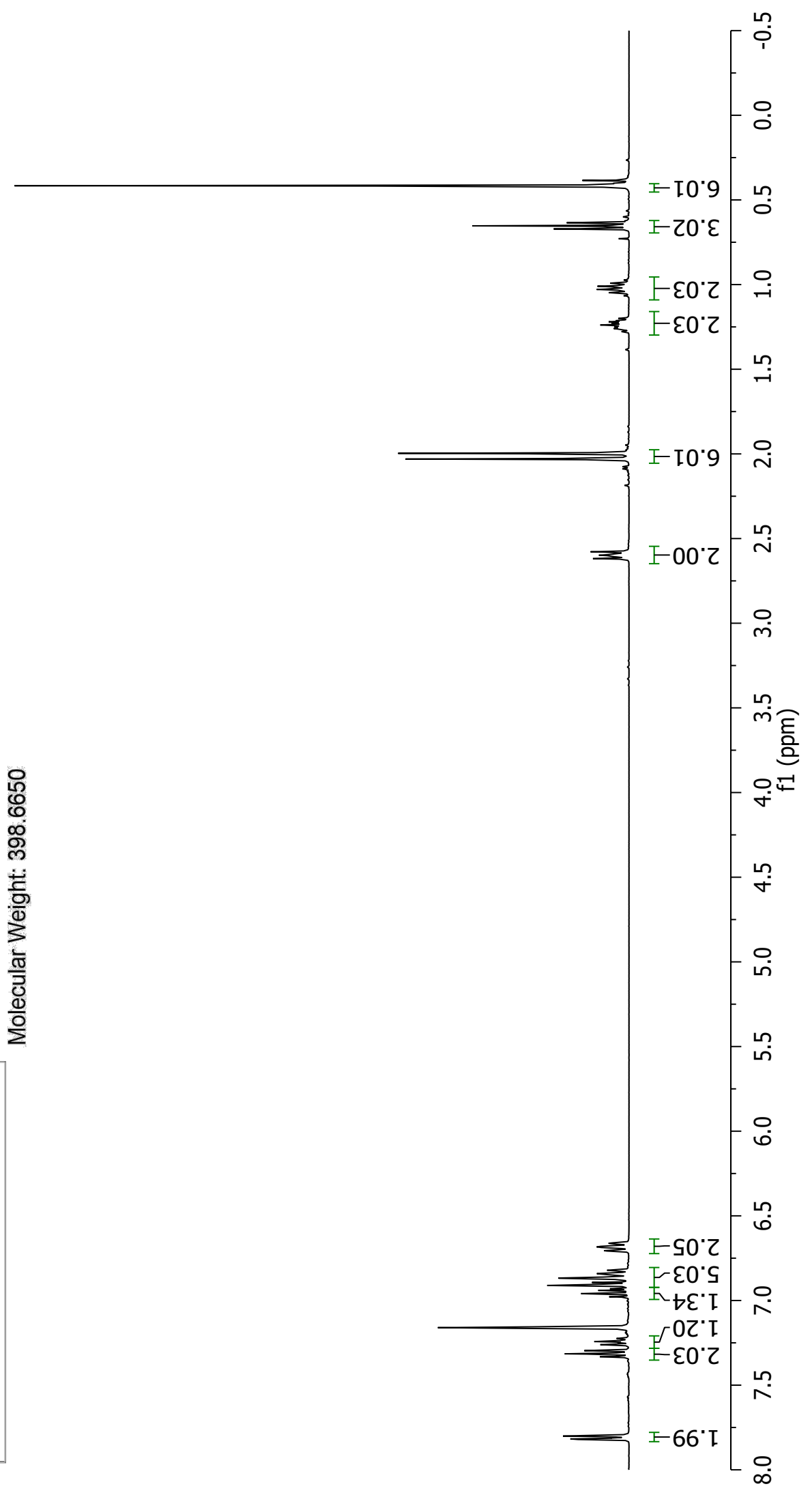




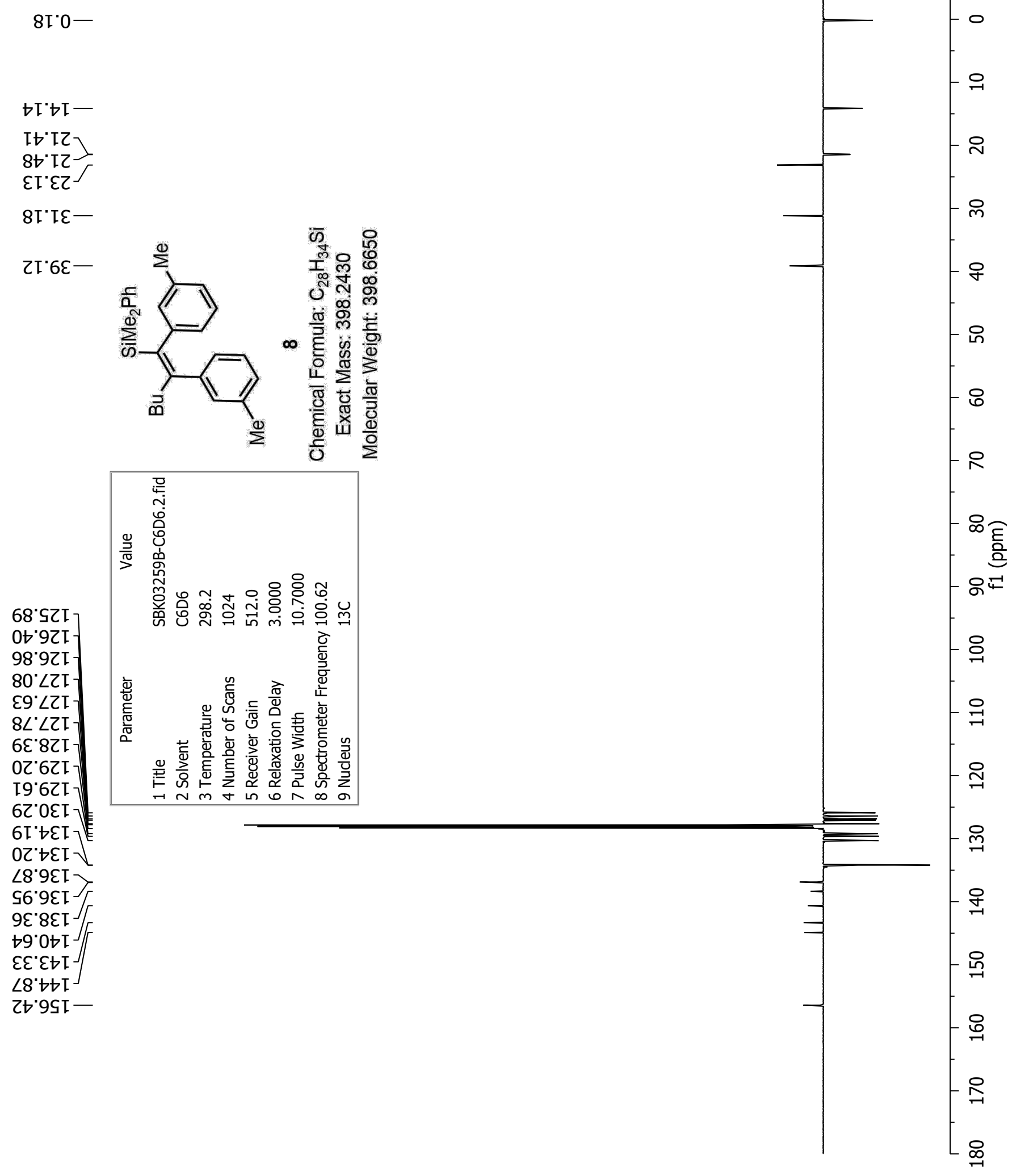



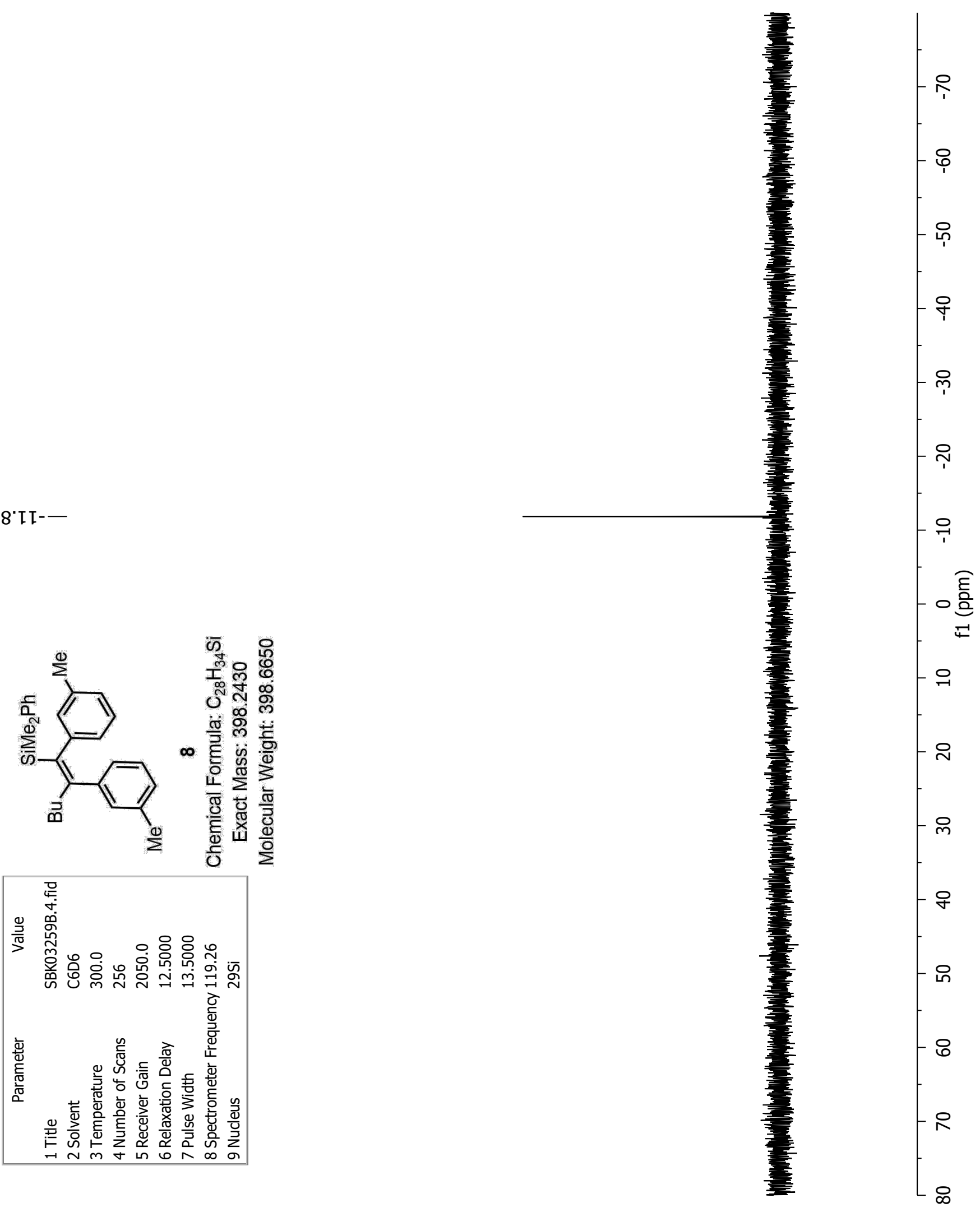


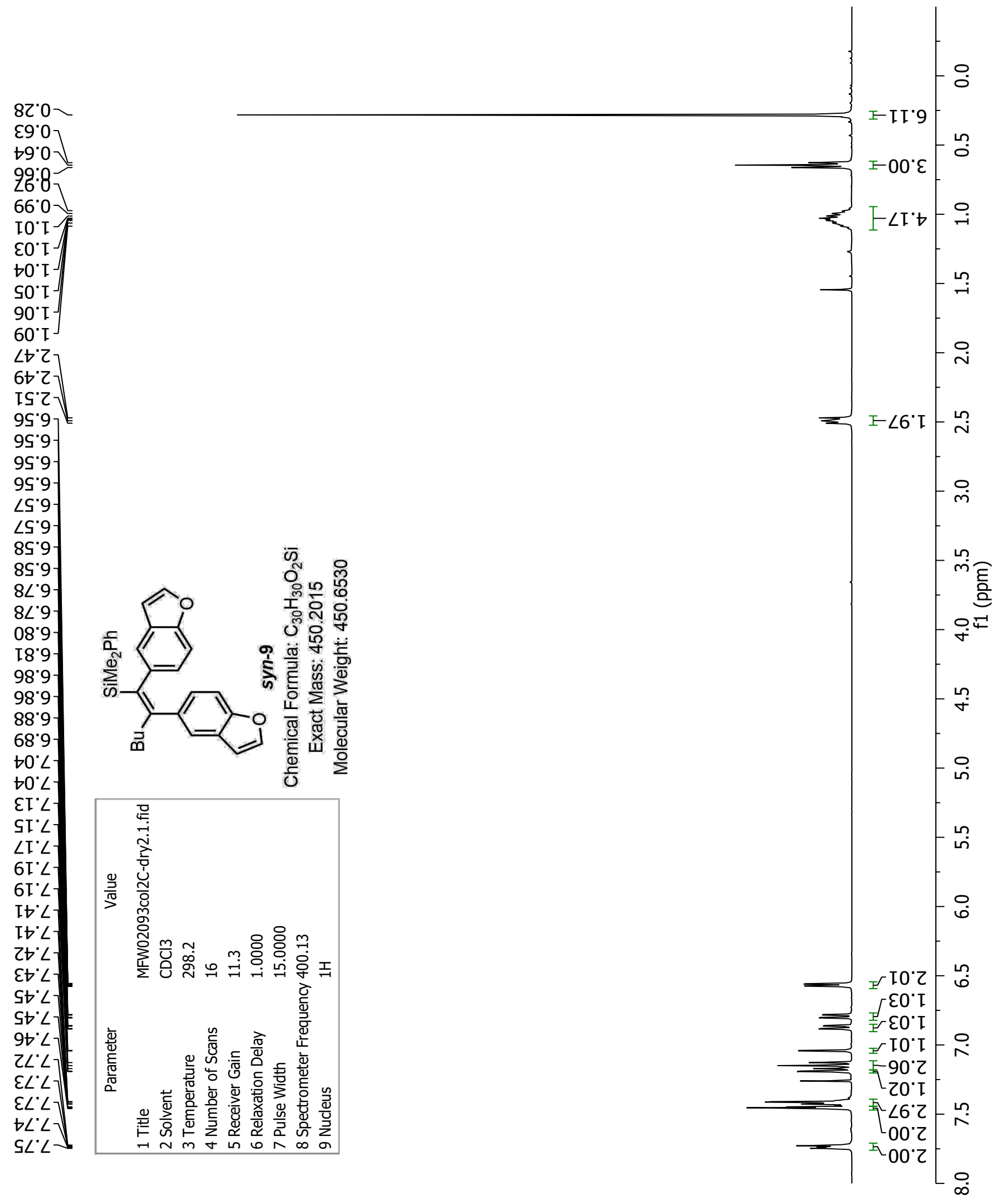



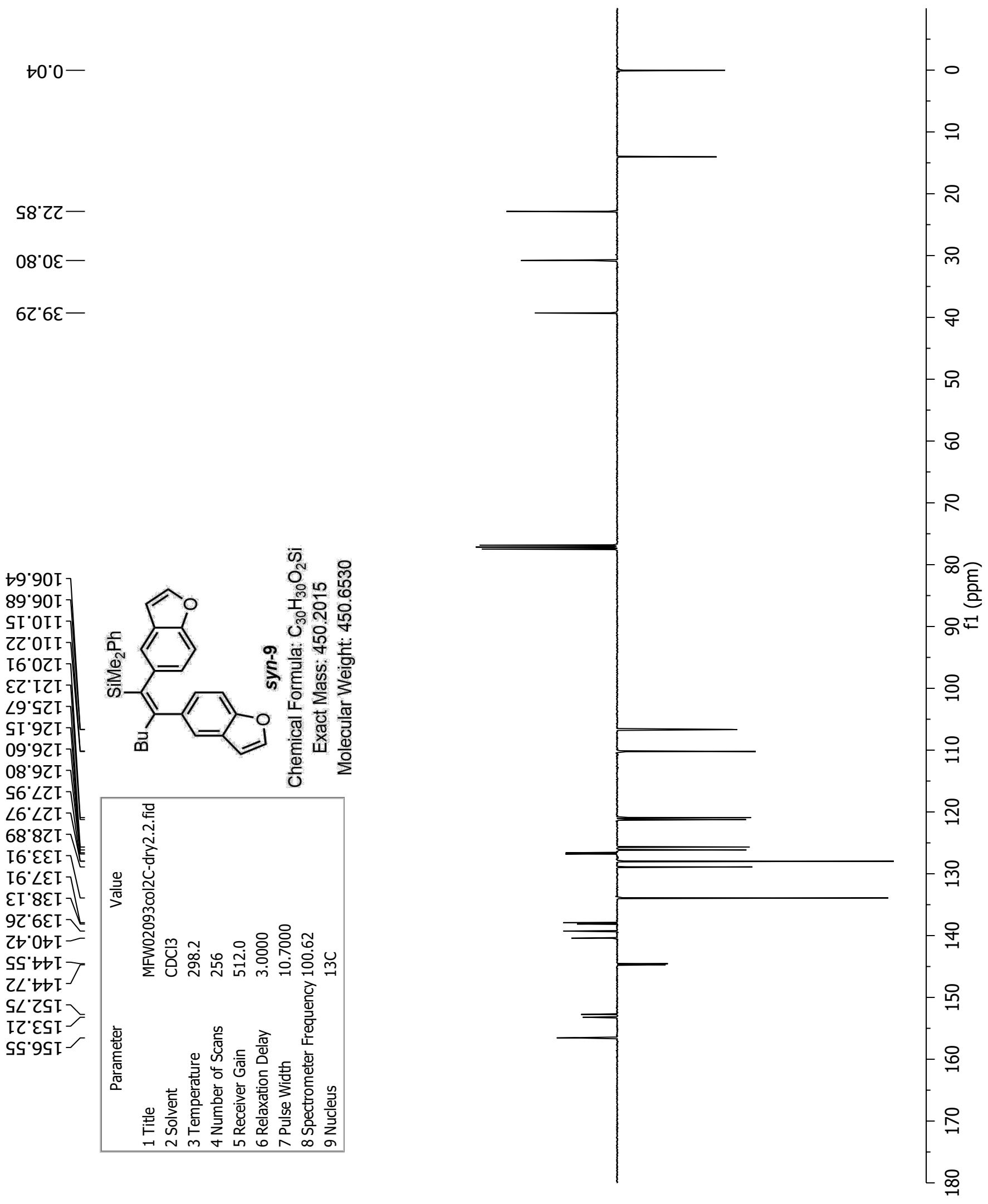


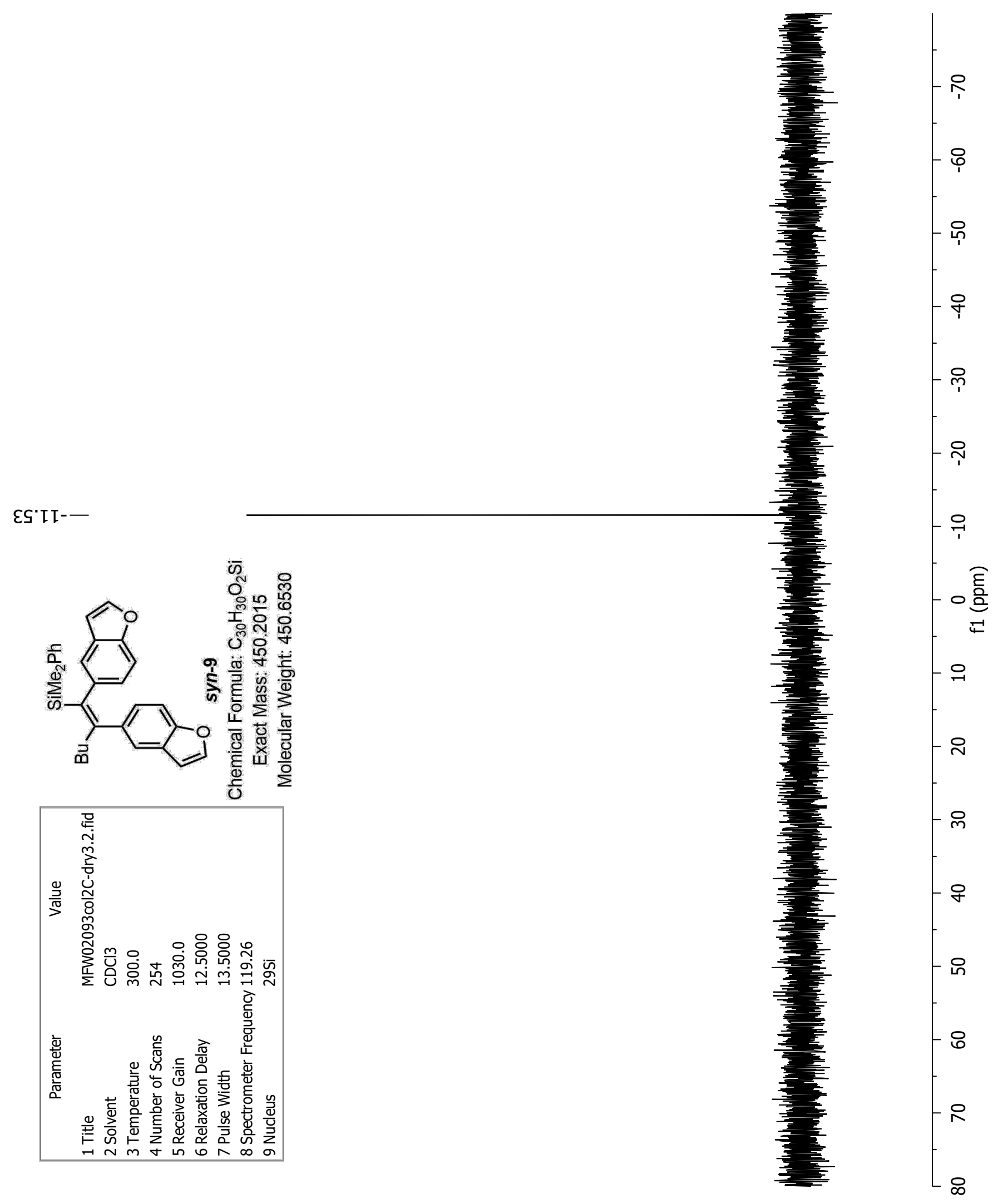


$85^{\circ} 0$

$65^{\circ} 0$

I9. 0

$96^{\circ} 0$

$\angle 6^{\circ} 0^{\circ}$

$66^{\circ} 0^{-}$

I0' I

ह0. I

$\angle O^{\circ} \mathrm{I}$.

60.

OI' $\mathrm{I}$

II'I-

ZI' I

9[' 2

$8 \mathrm{I}^{\circ} \mathrm{Z}$

$0 Z^{\prime} \mathrm{Z}$
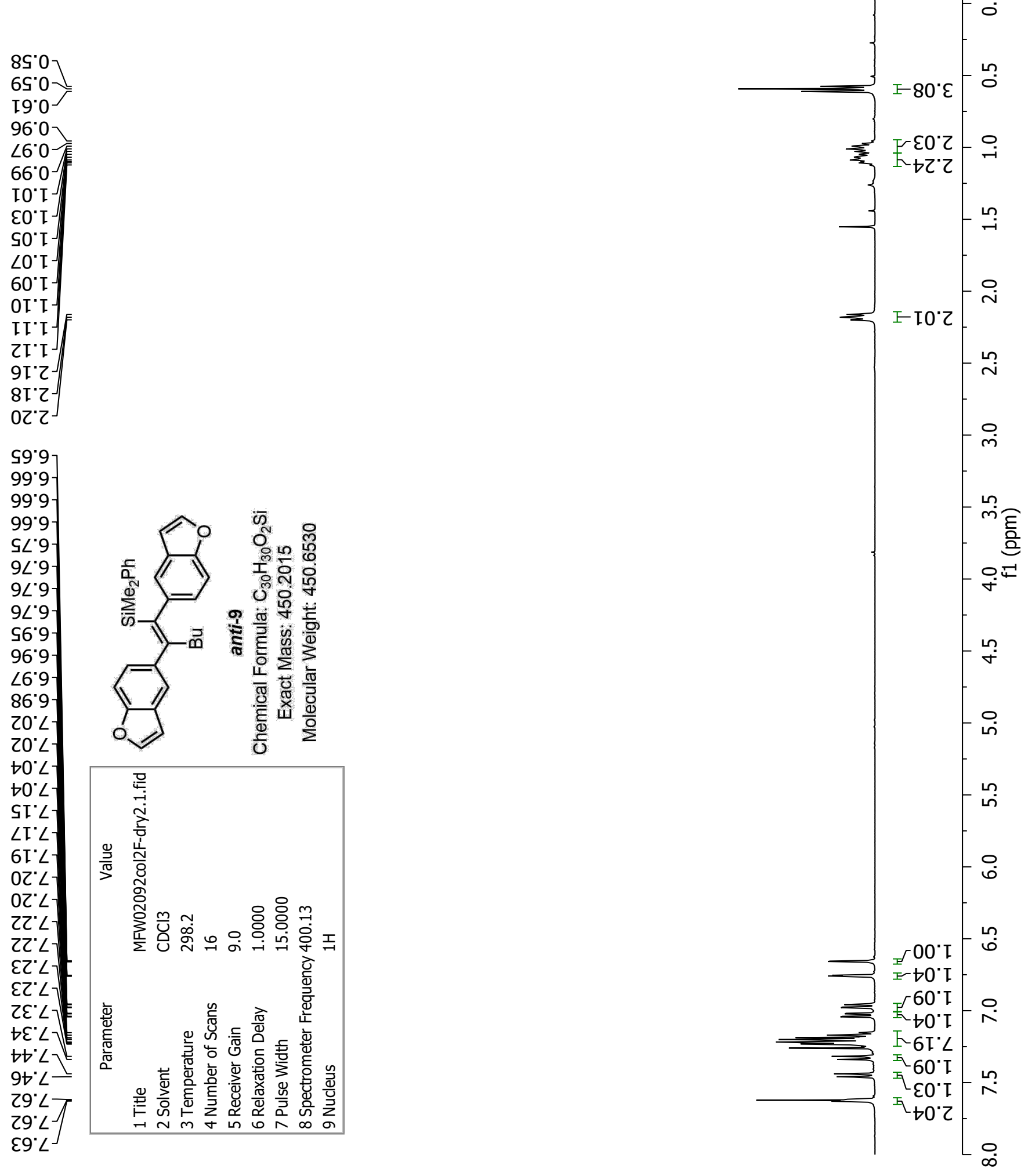

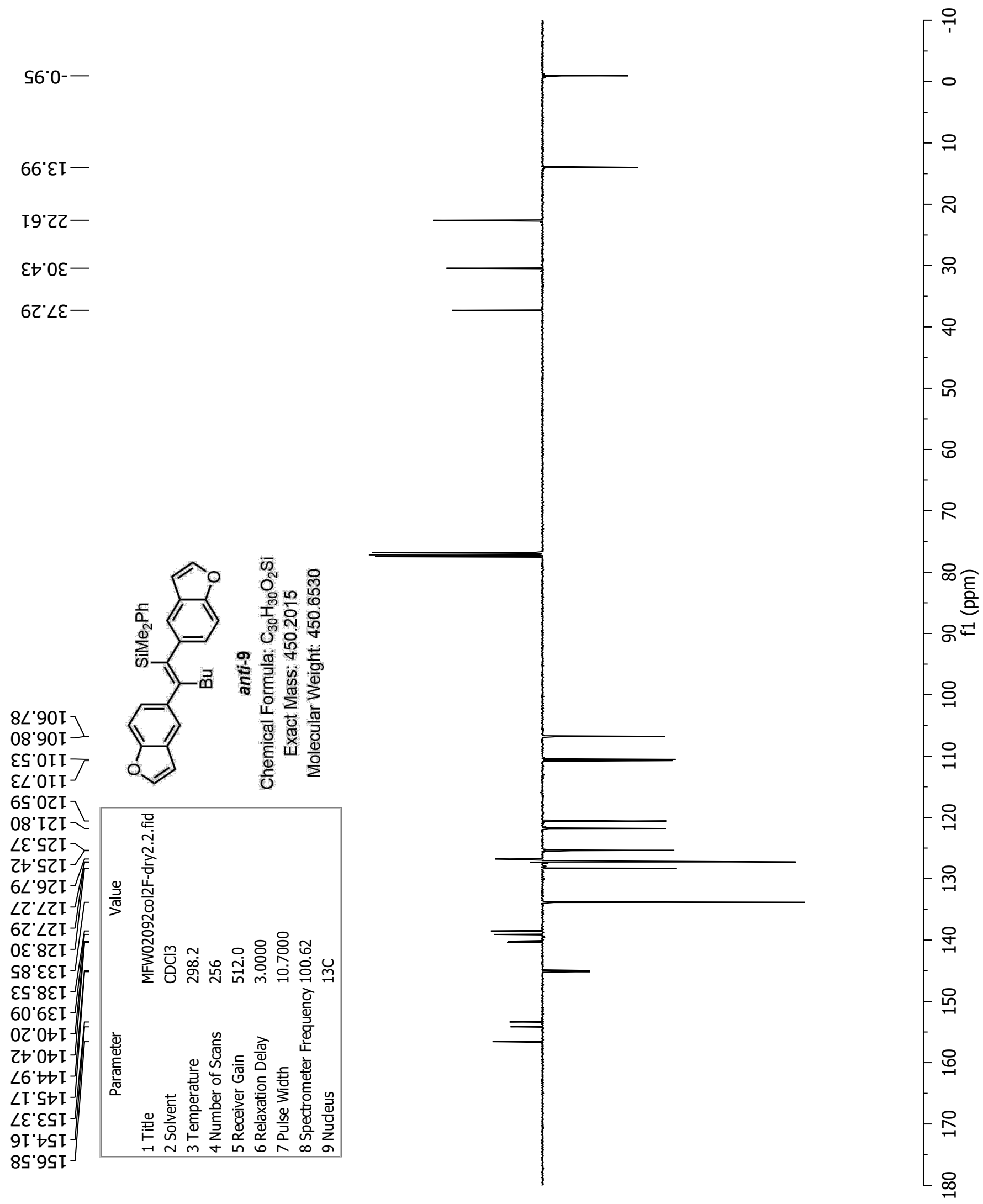


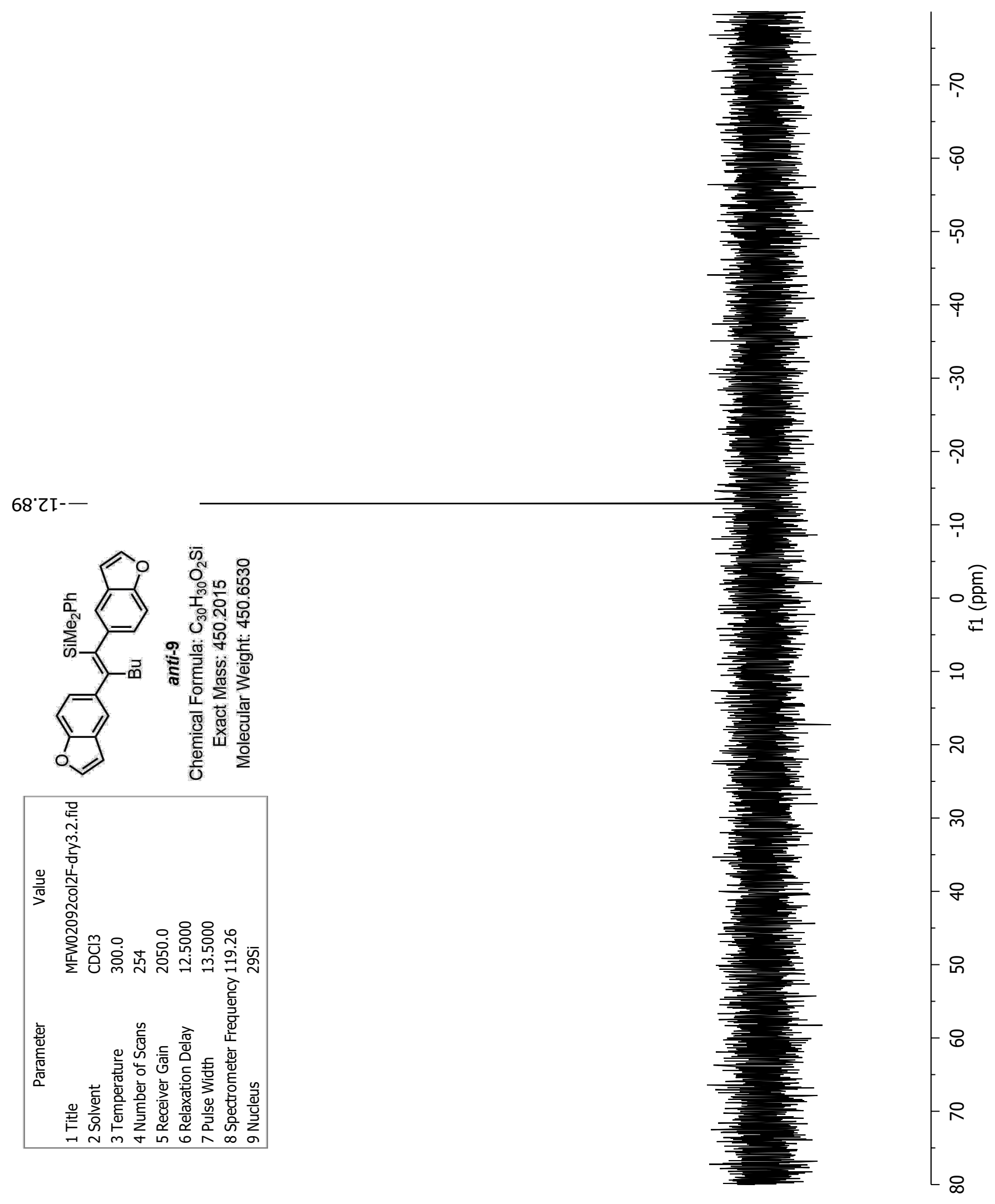


${ }^{\circ} \varepsilon^{\circ}-$

† ${ }^{\circ} \varepsilon-$

$9 \angle{ }^{\circ} 9$

$\angle \angle ' 9$

$8 \angle ' 9$

ع8.9-

ร8.9.

$\angle 8^{\circ} 9$

$88^{\circ} 9$

$68^{\circ} 9$

68.9-

$06^{\circ} 9$

Z6.9-

ع6.9-

ธ6.9-

$96^{\circ} 9$

t0 $\angle$

$90^{\circ} \mathrm{L}$

$90^{\circ} \angle$

$80^{\circ} \mathrm{L}$

$\left.0 T^{\circ} \angle\right]$

$\left.6 \varepsilon^{\circ} \angle\right]$

$\left.\begin{array}{l}0 t^{\circ} \angle \\ I t^{\circ} \angle\end{array}\right]$

$69^{\circ} \angle$

$\left.69^{\circ} \angle\right]$

$0 \angle \angle Y$
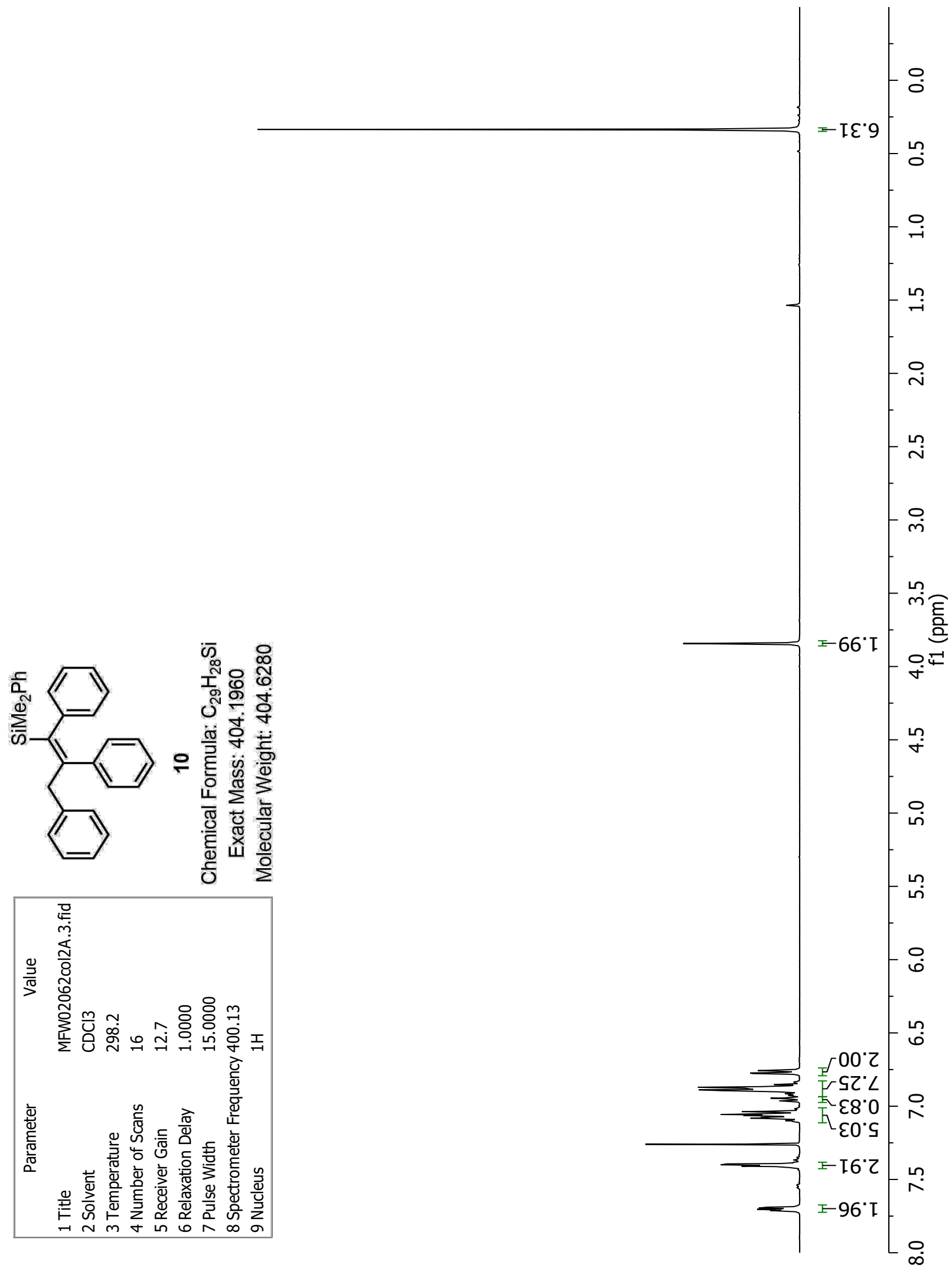

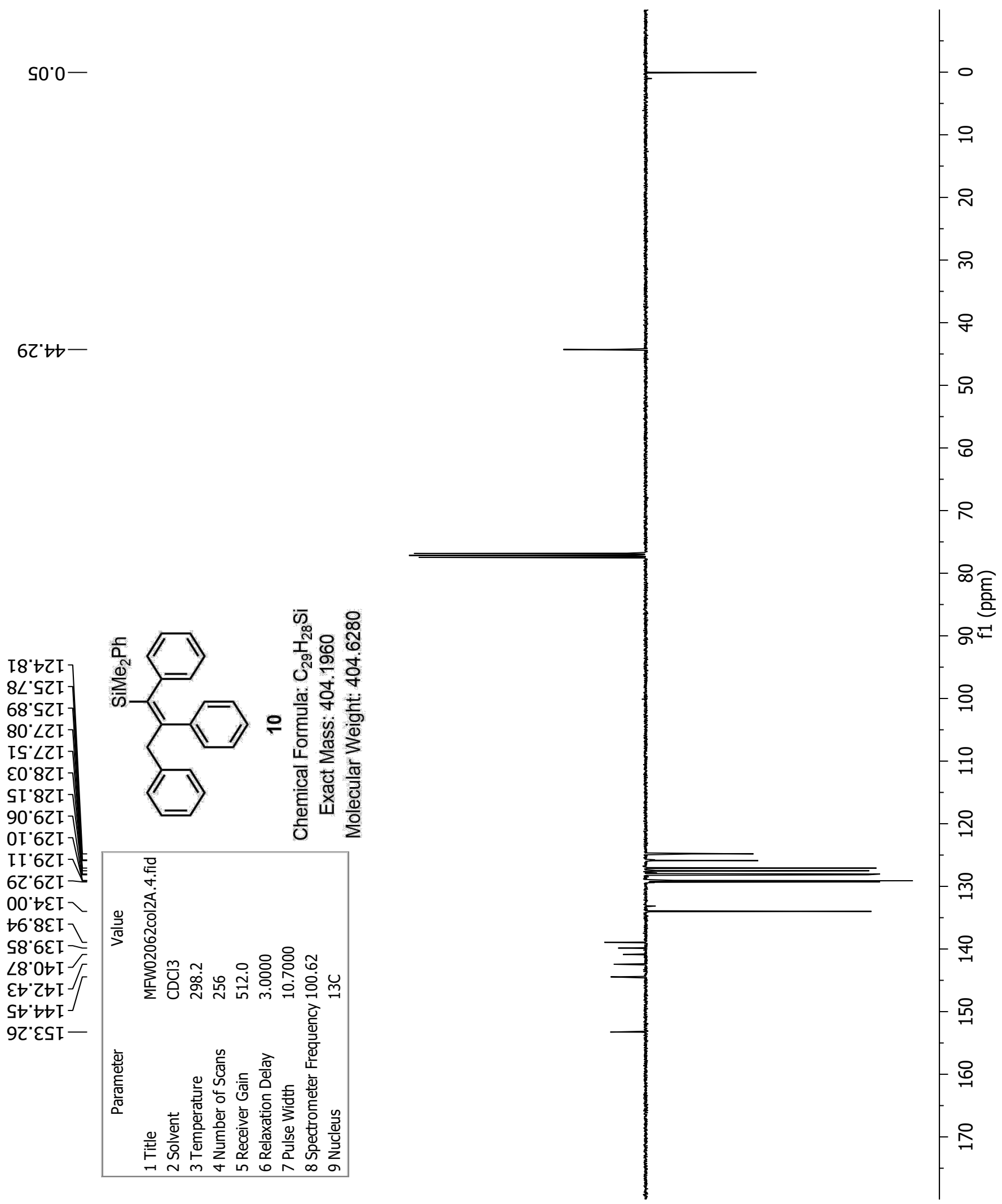
$8 \varepsilon^{*}$ II-
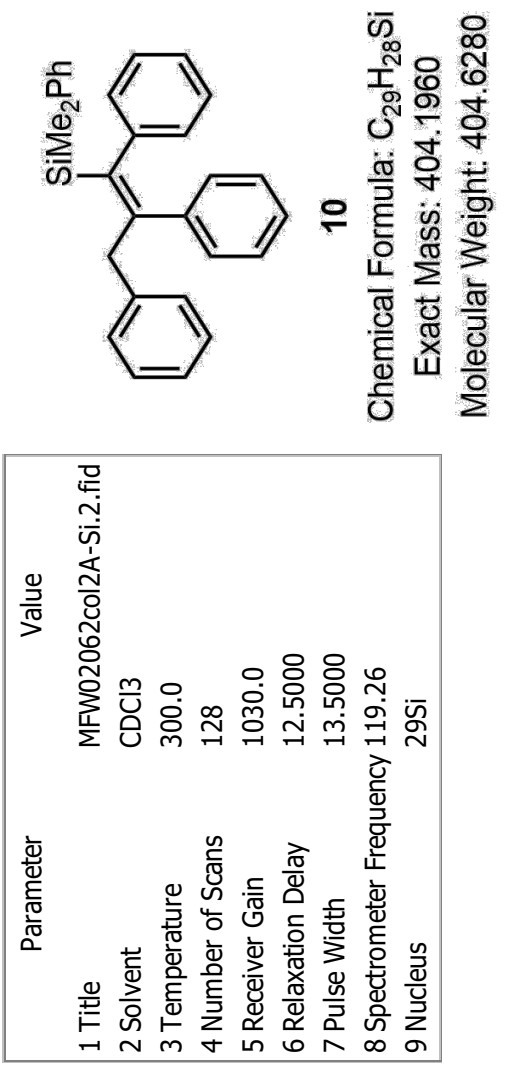

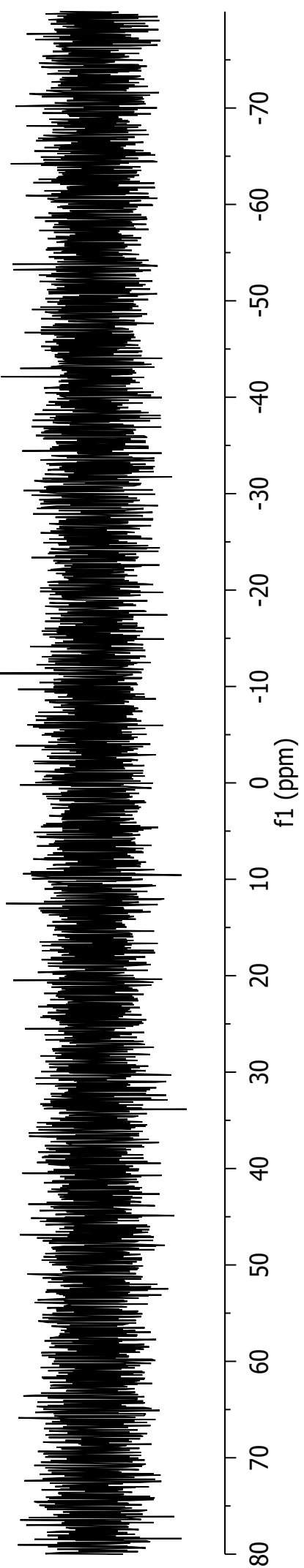




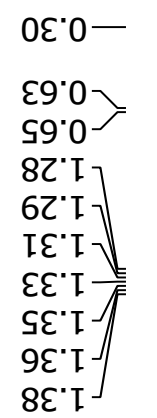

$6 \varepsilon \cdot 2$
Iナて

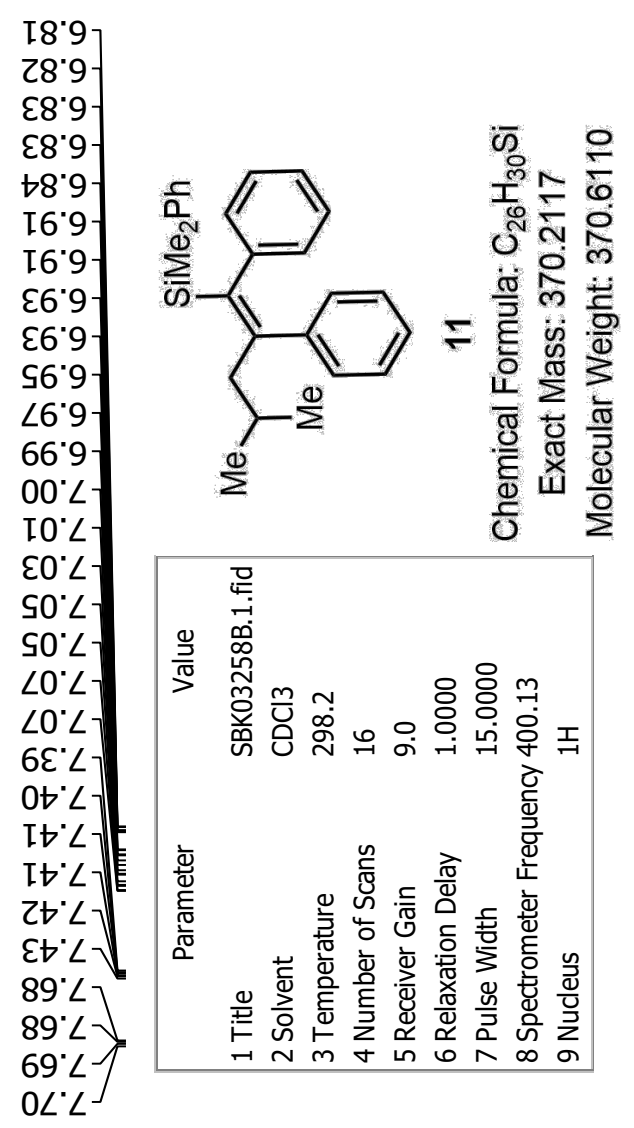

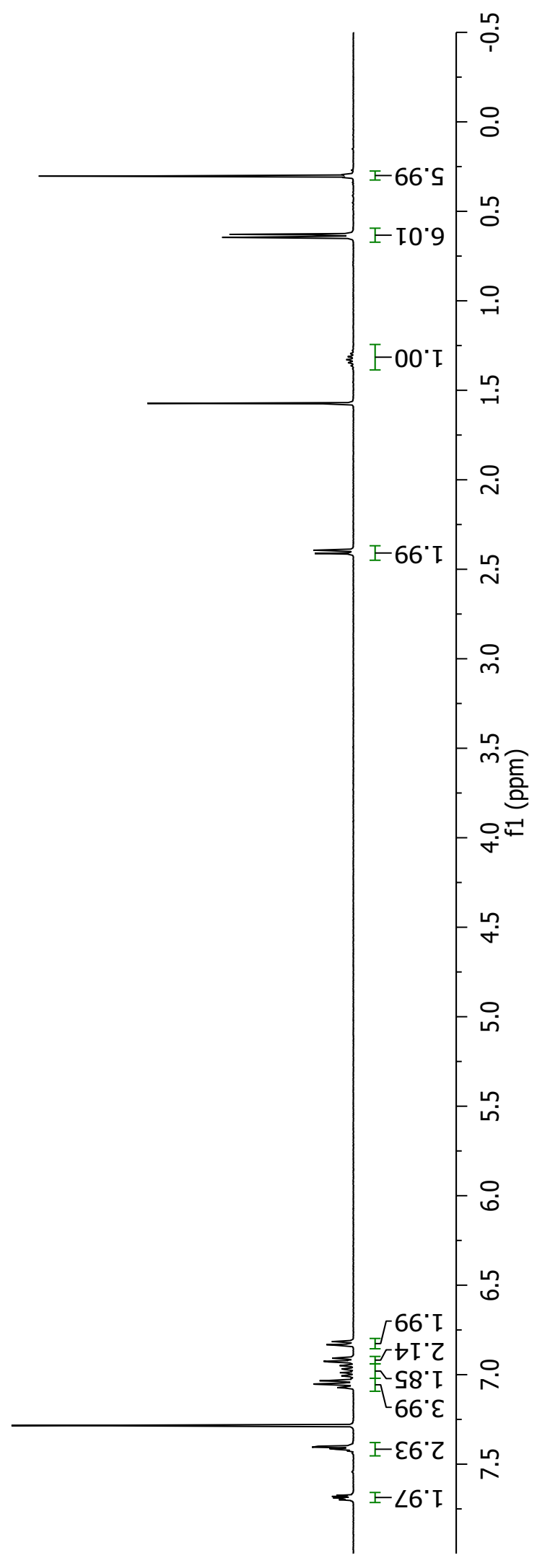




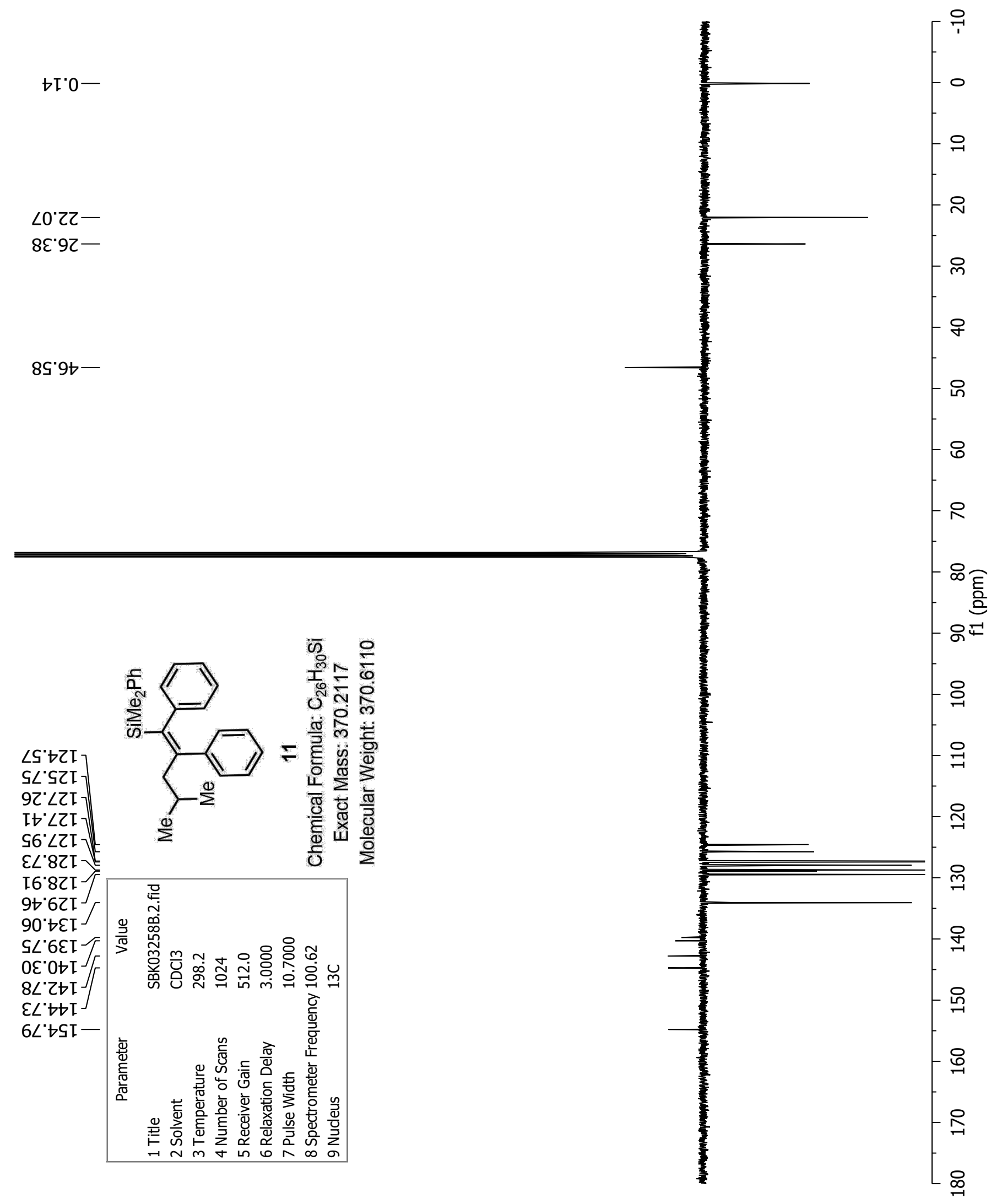



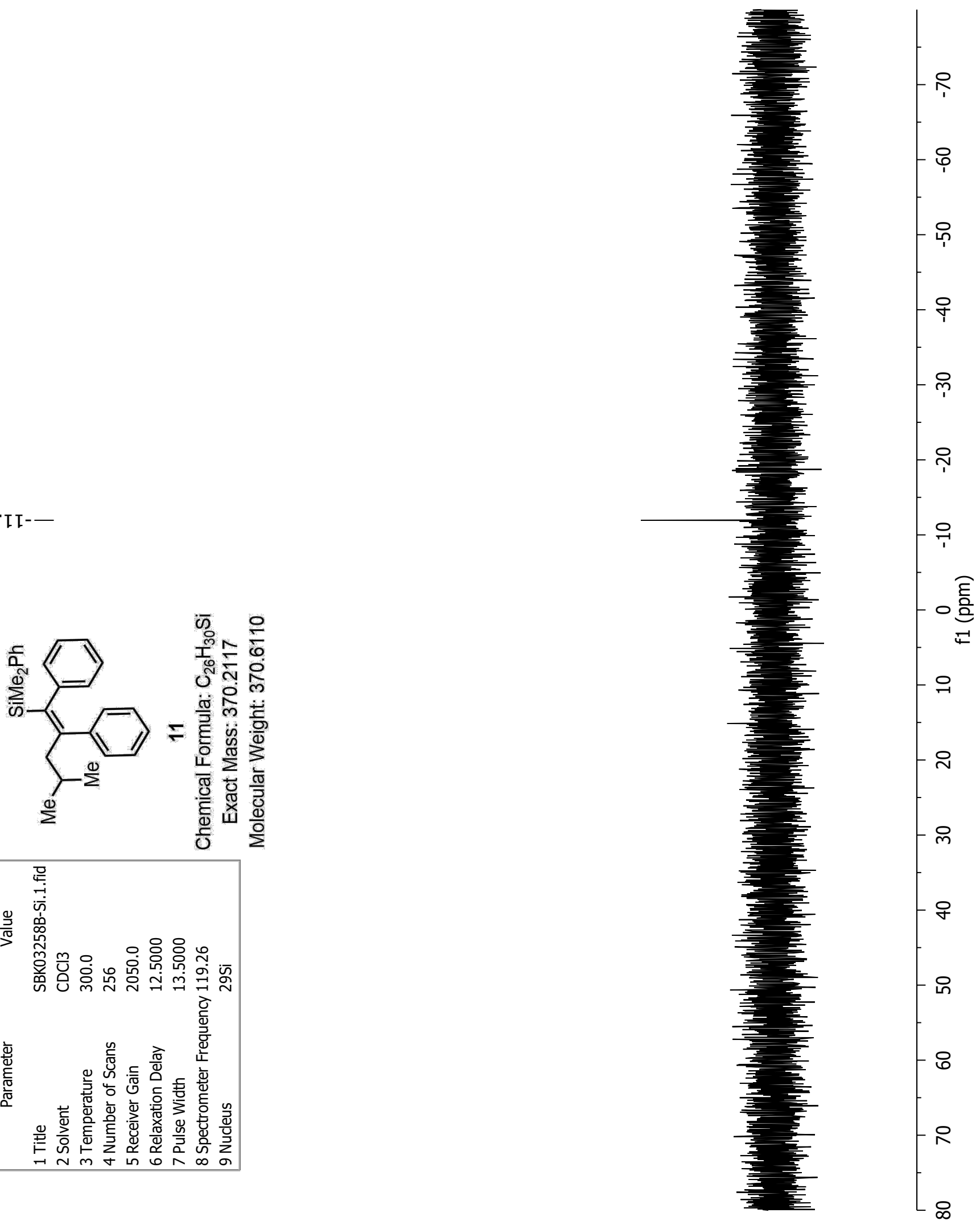

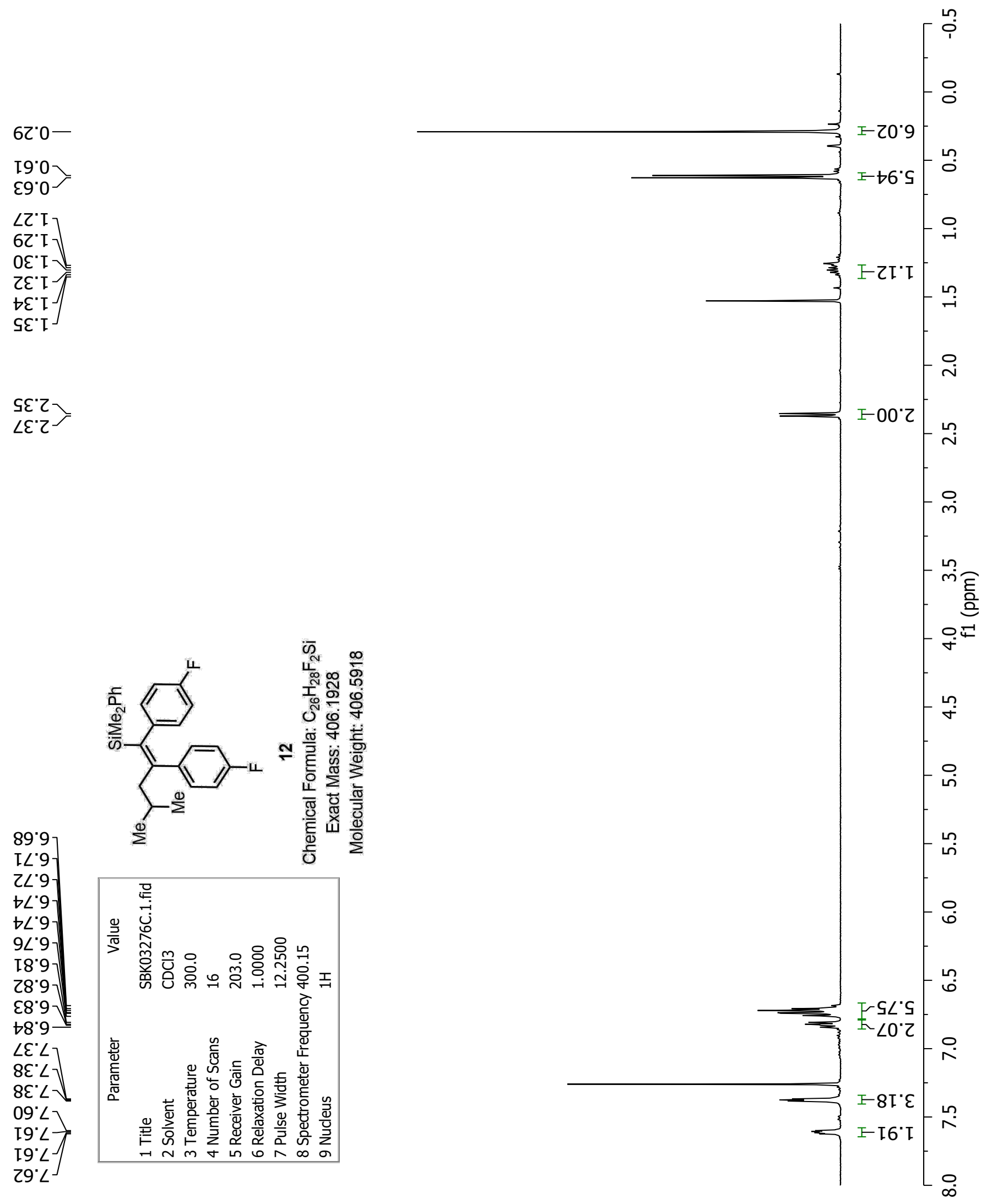


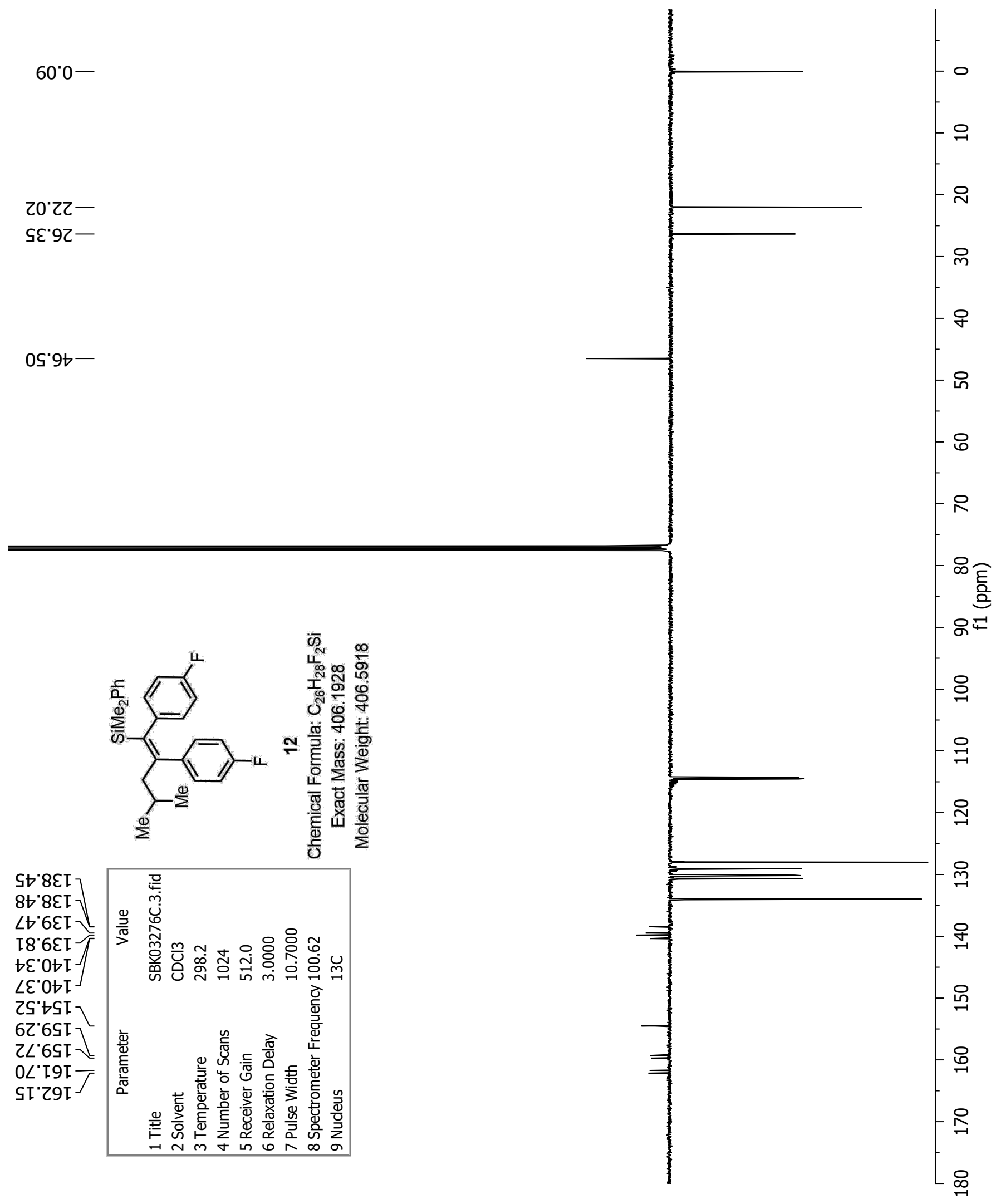



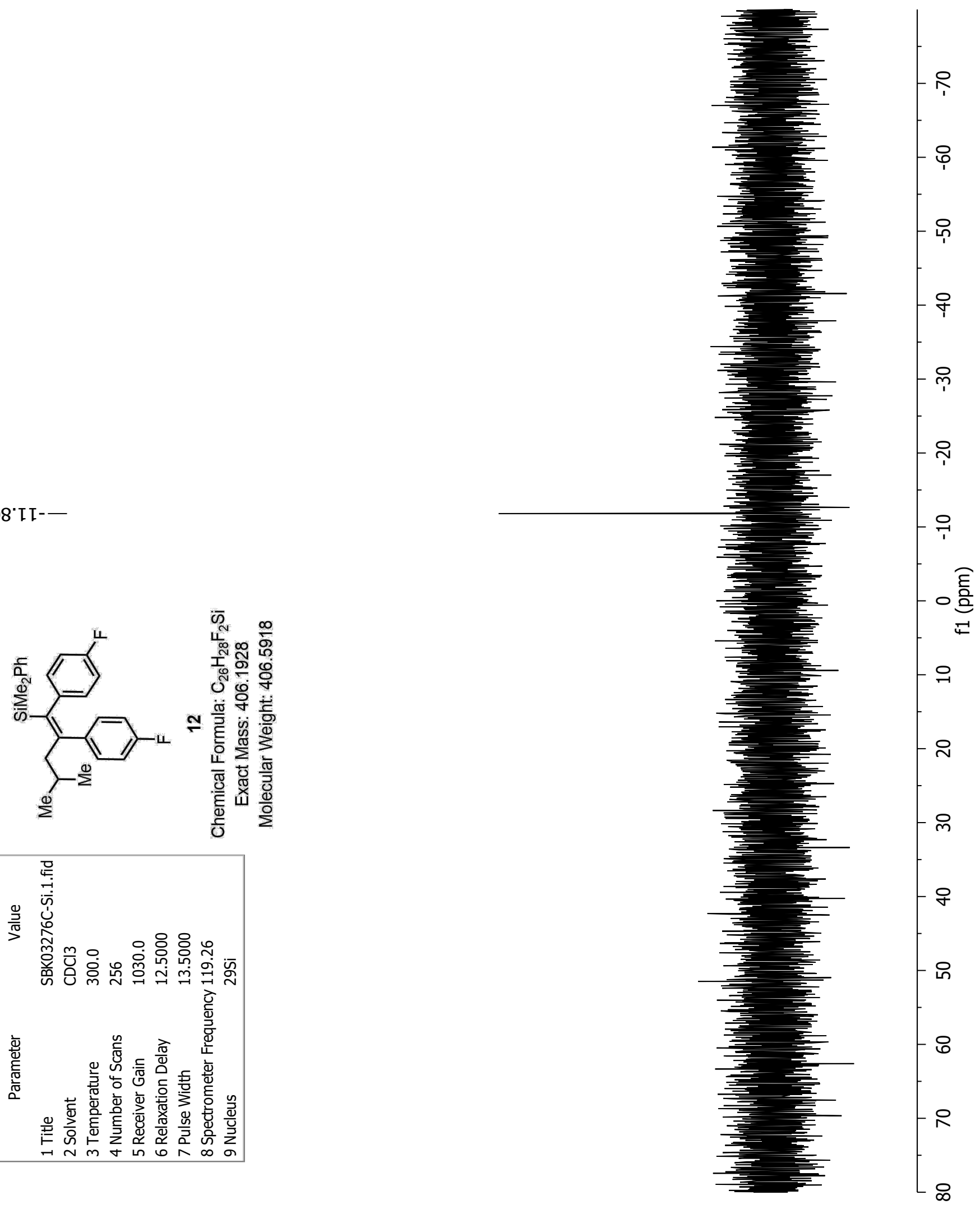


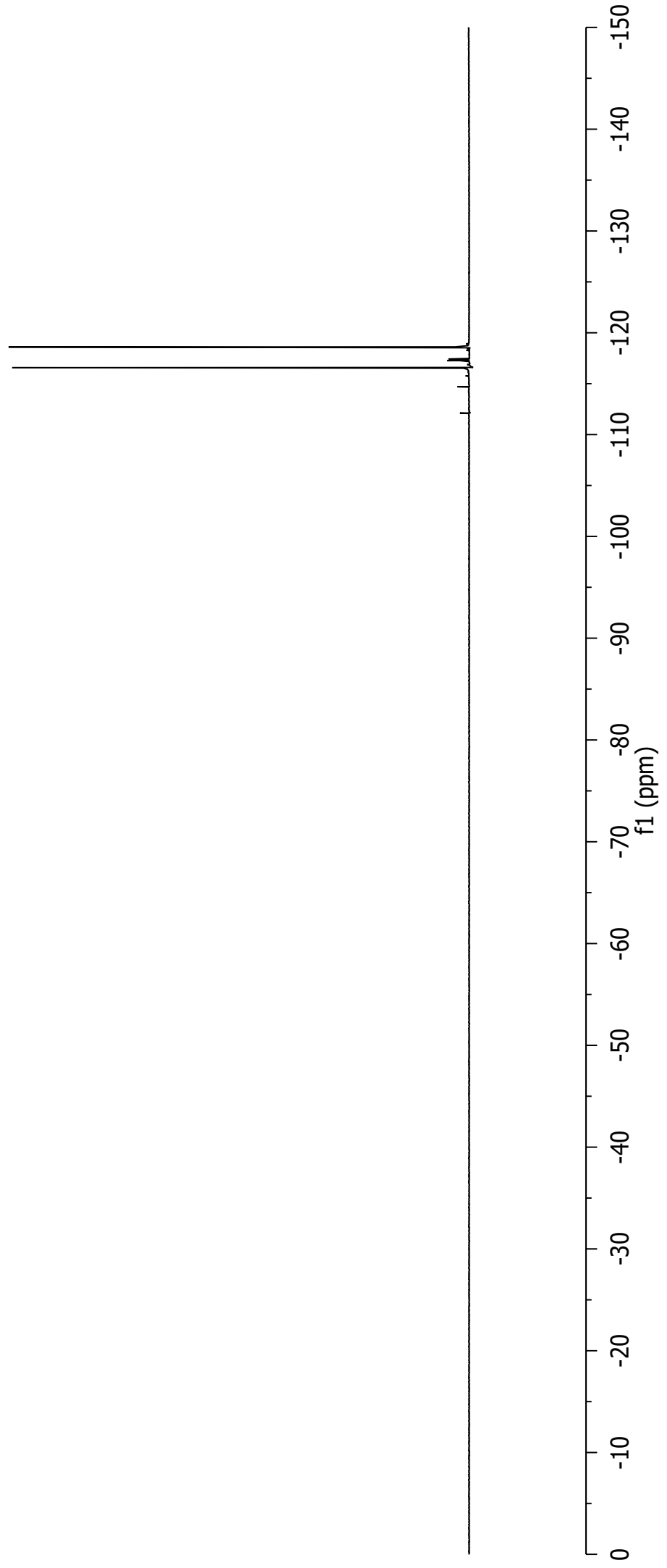




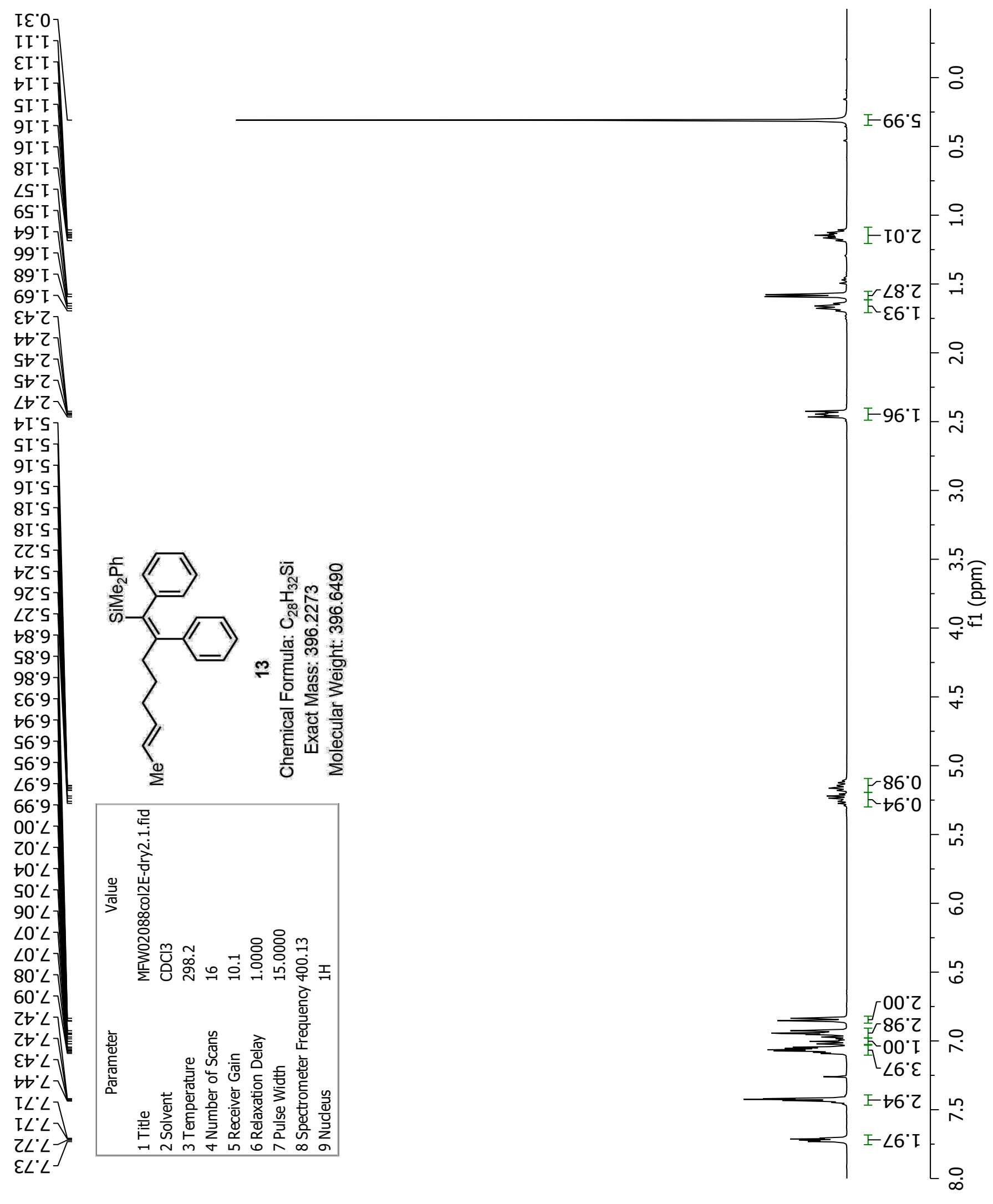



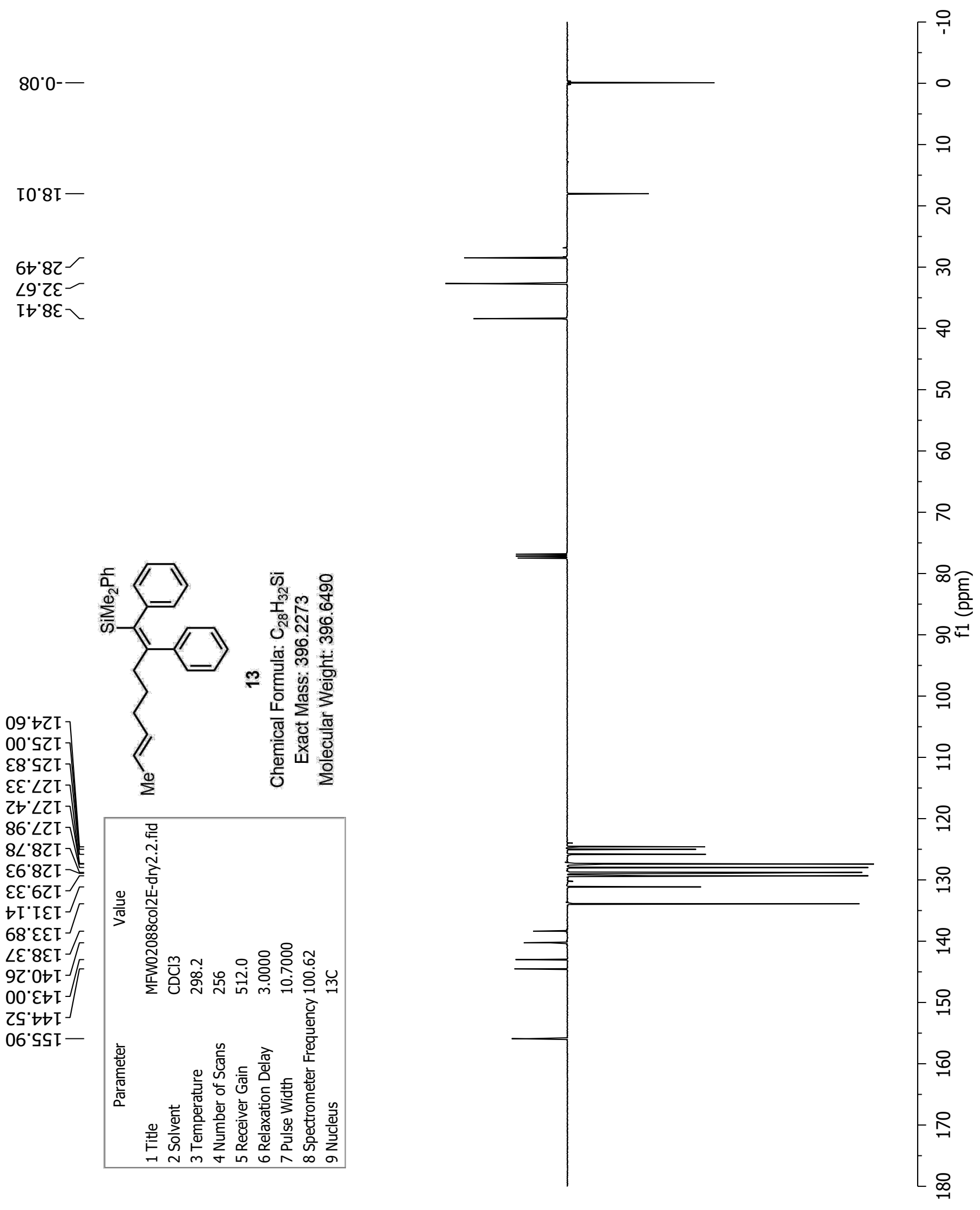


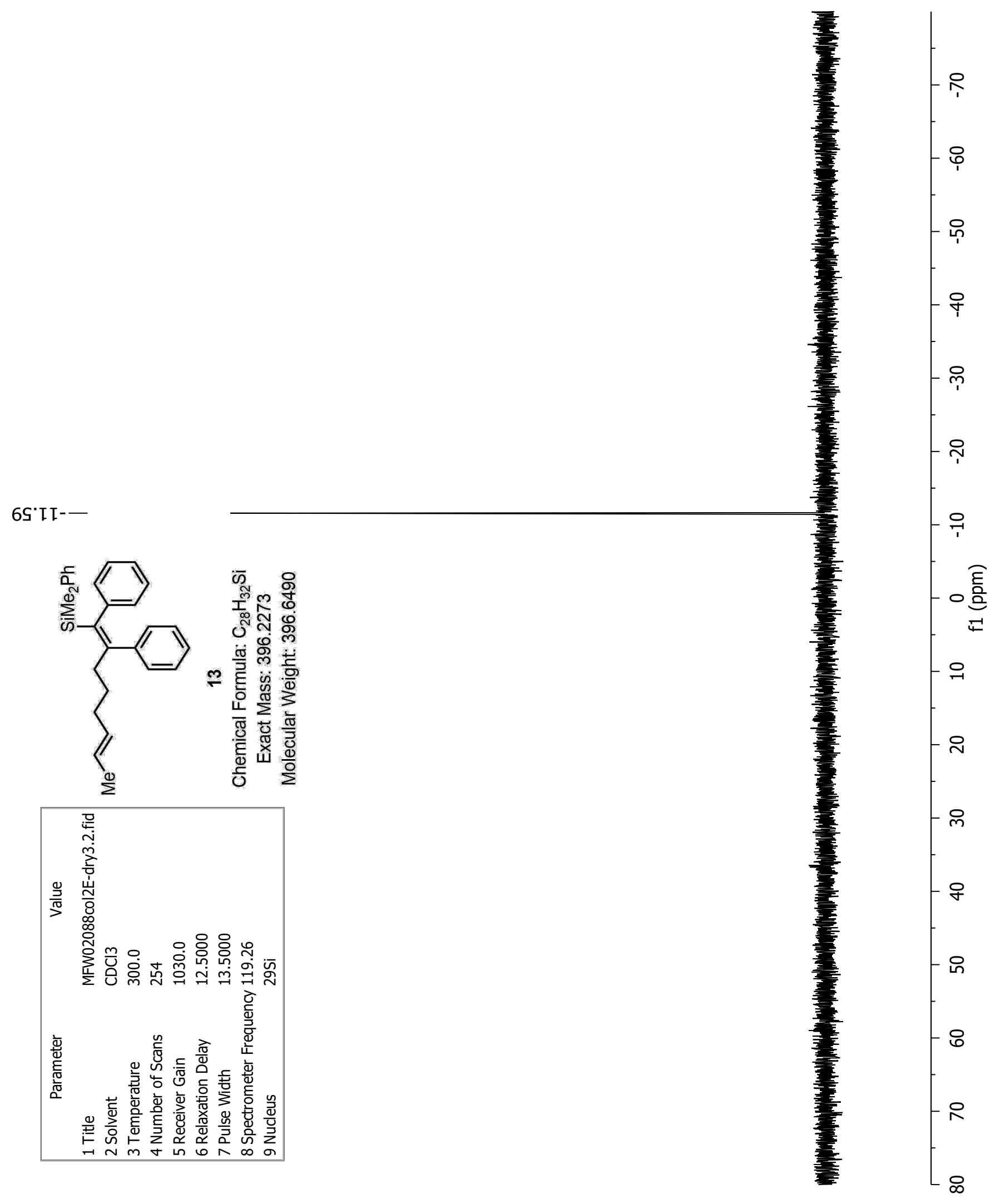



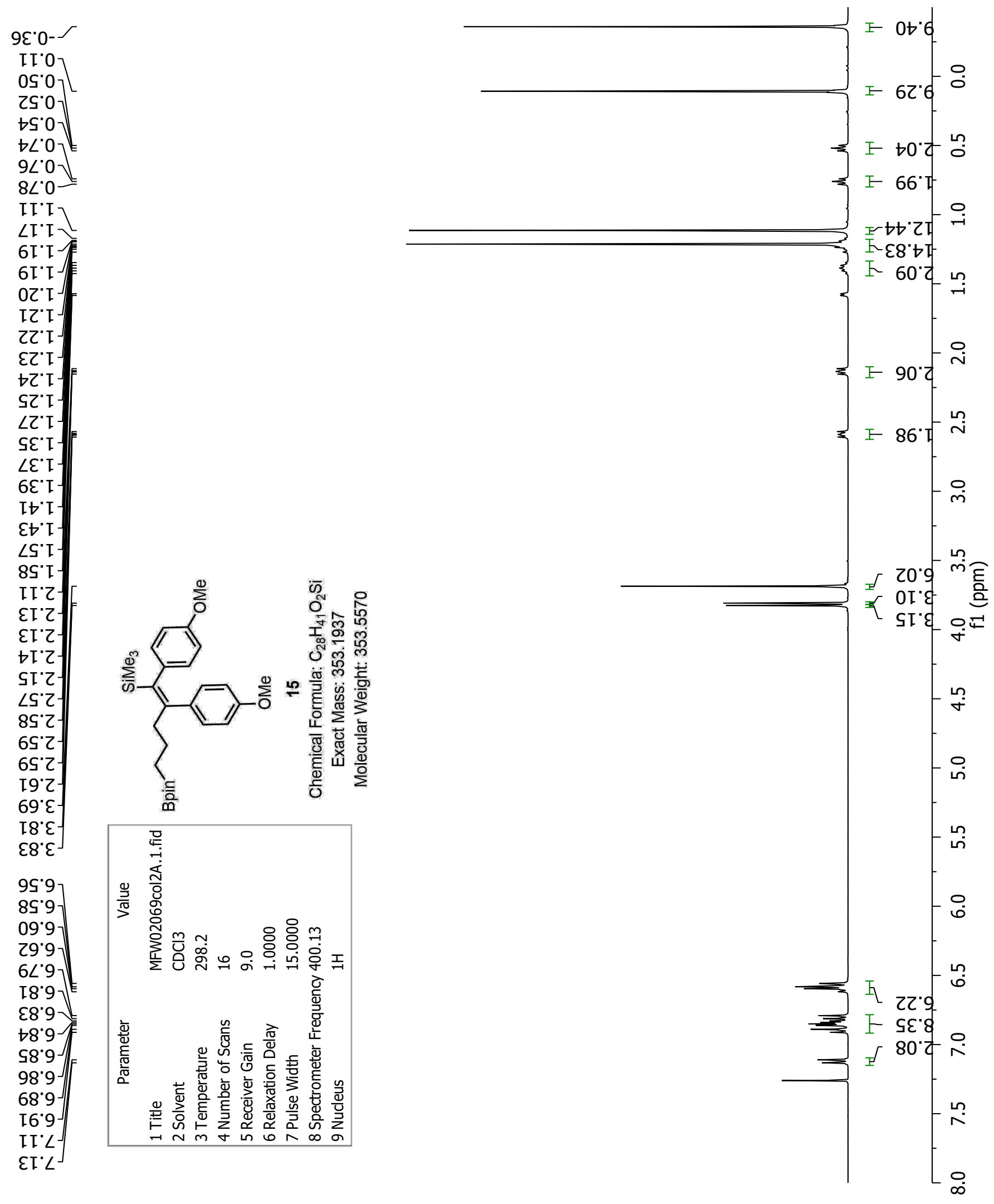


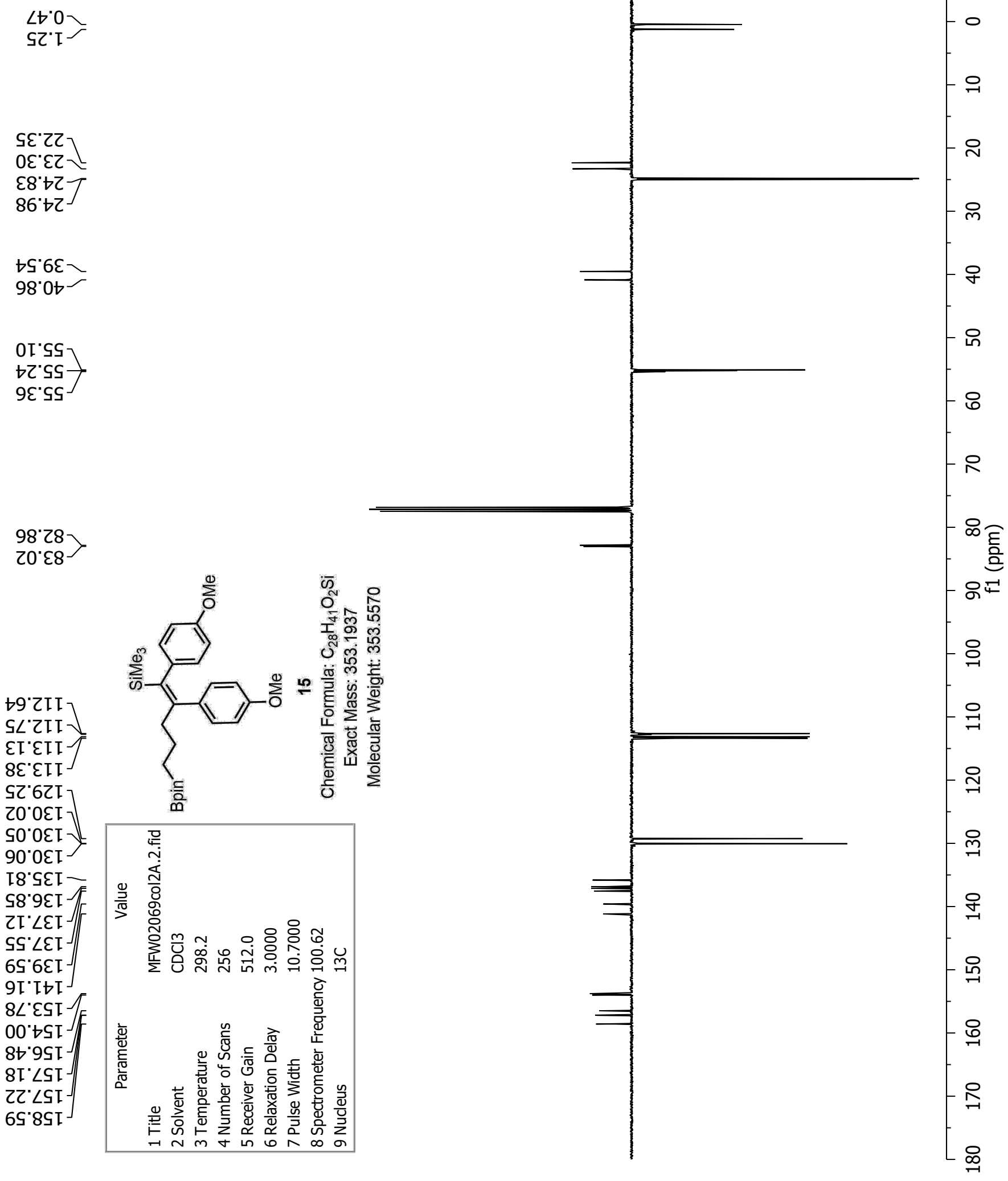


6L'L-
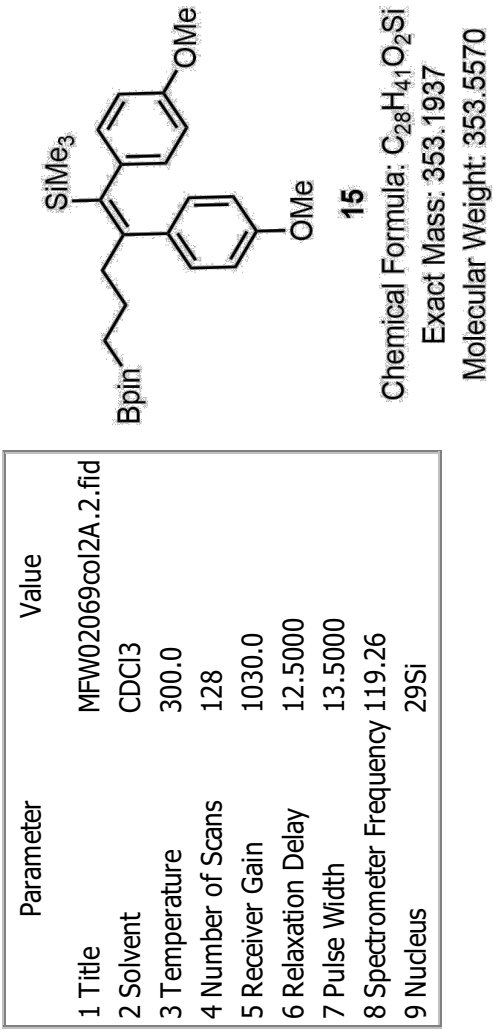

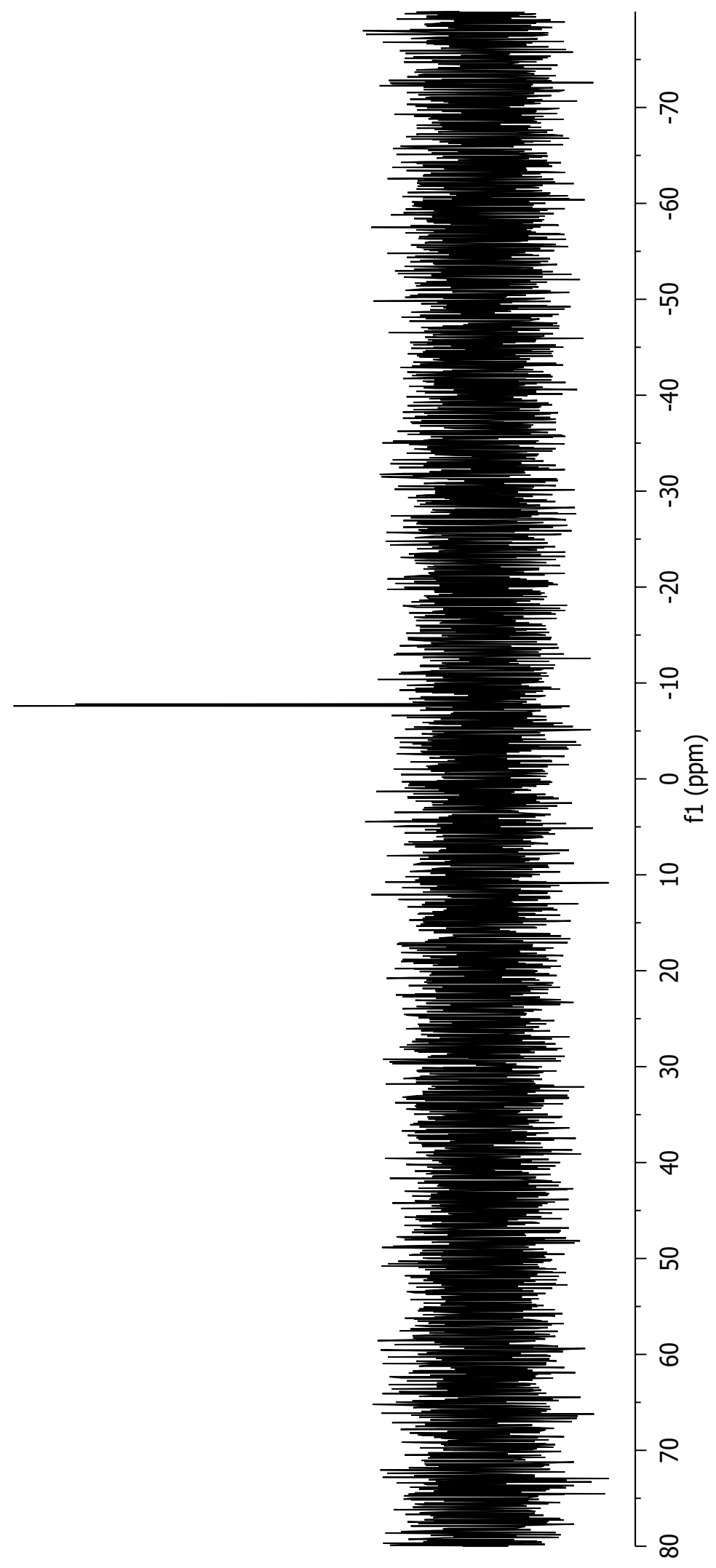



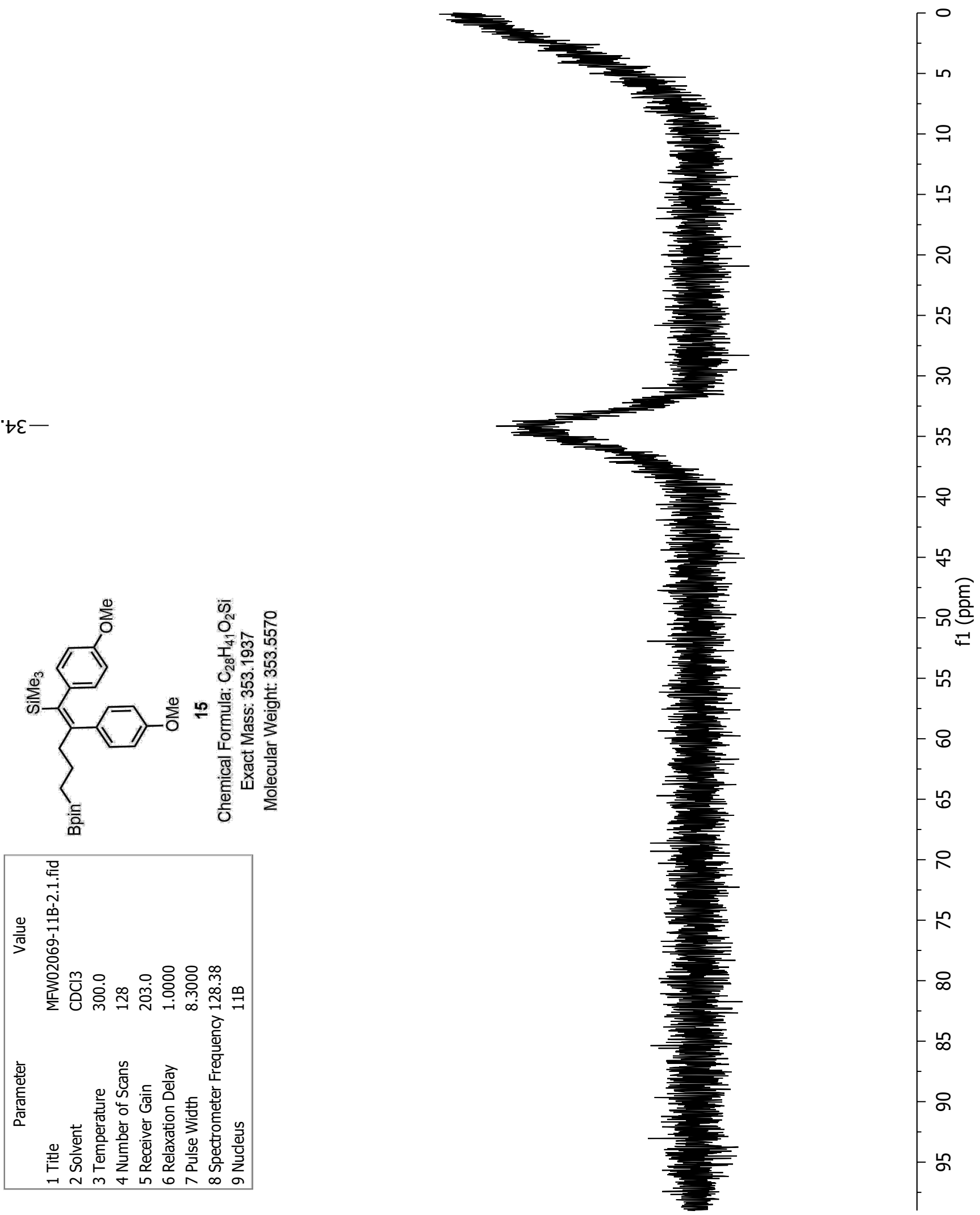


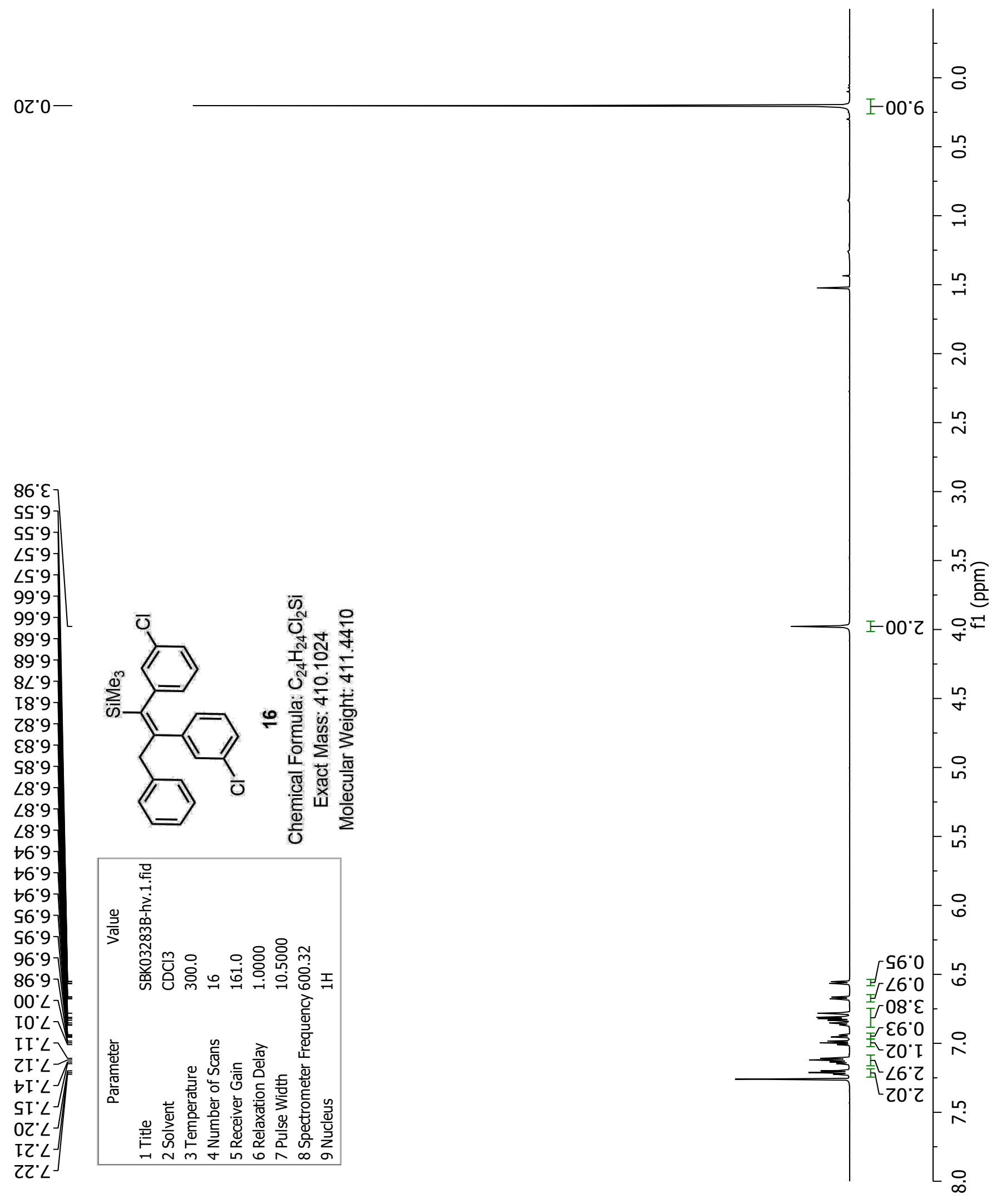




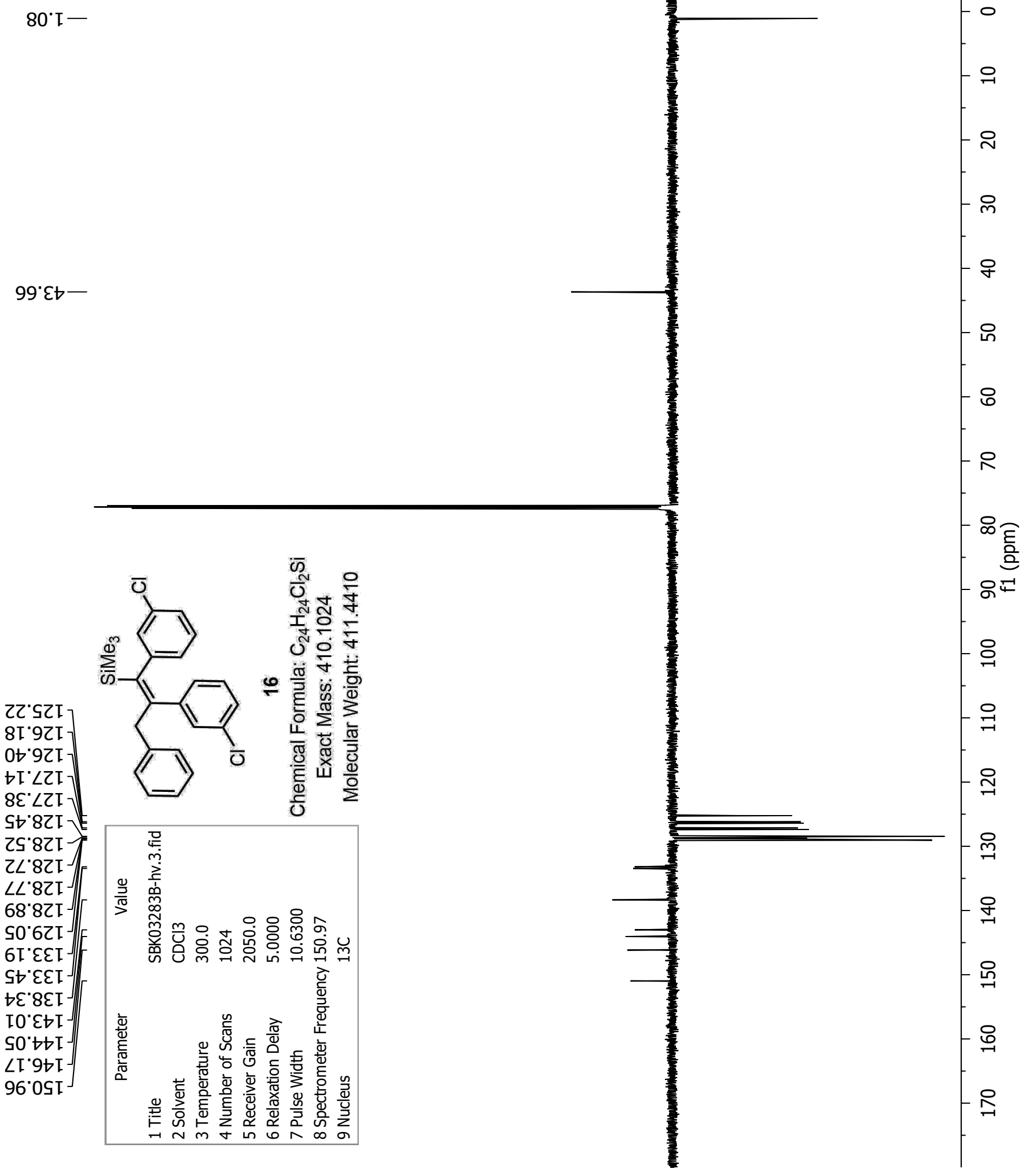


9t'9--

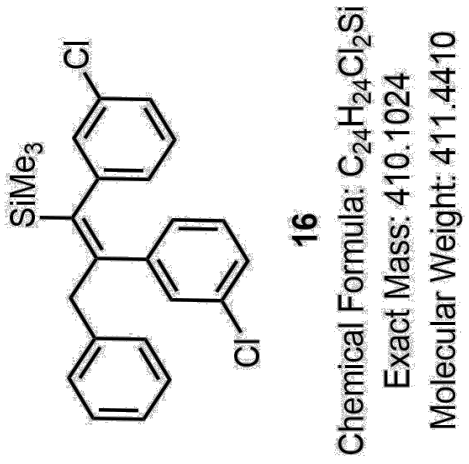

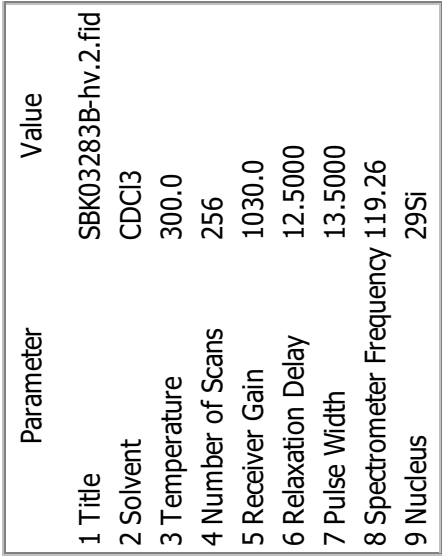

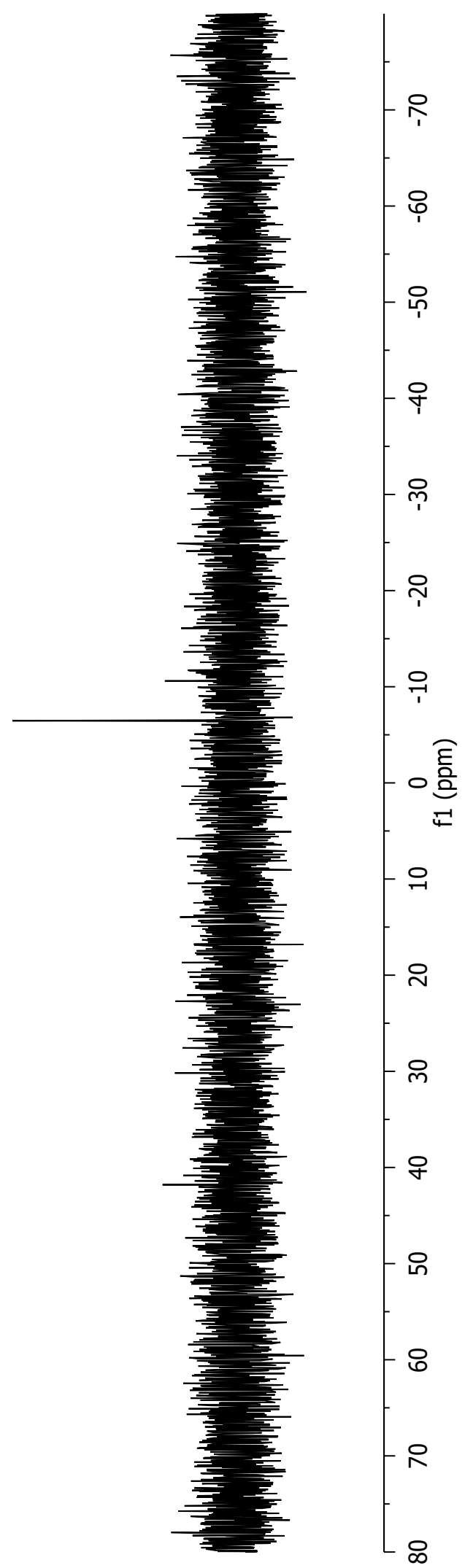



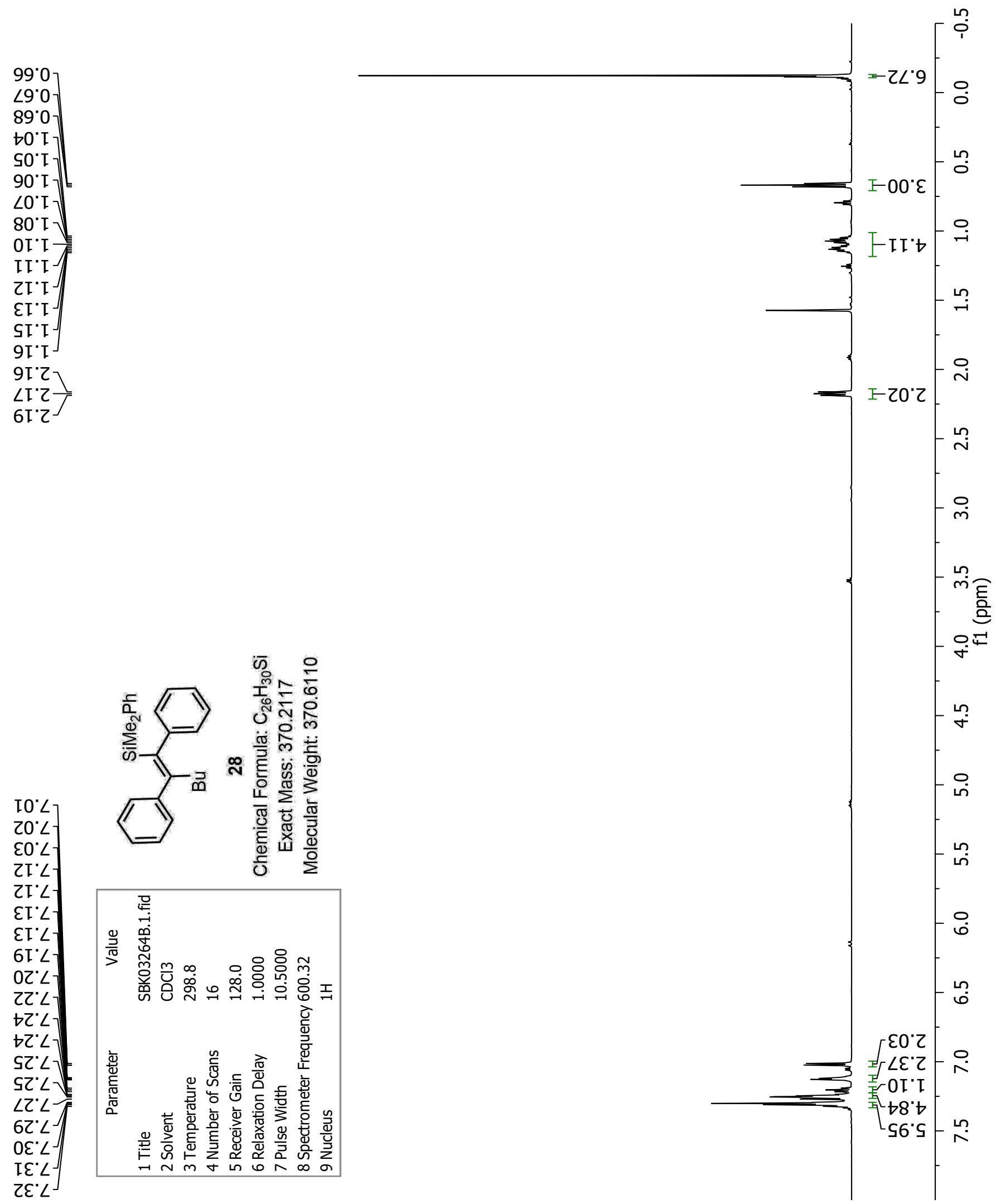
$\angle 6^{\circ} 0^{-}-$

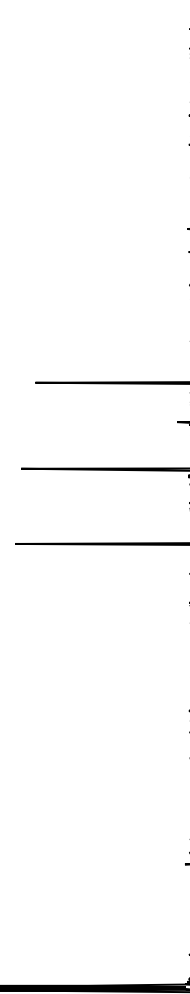

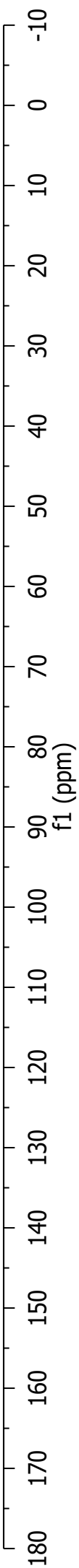




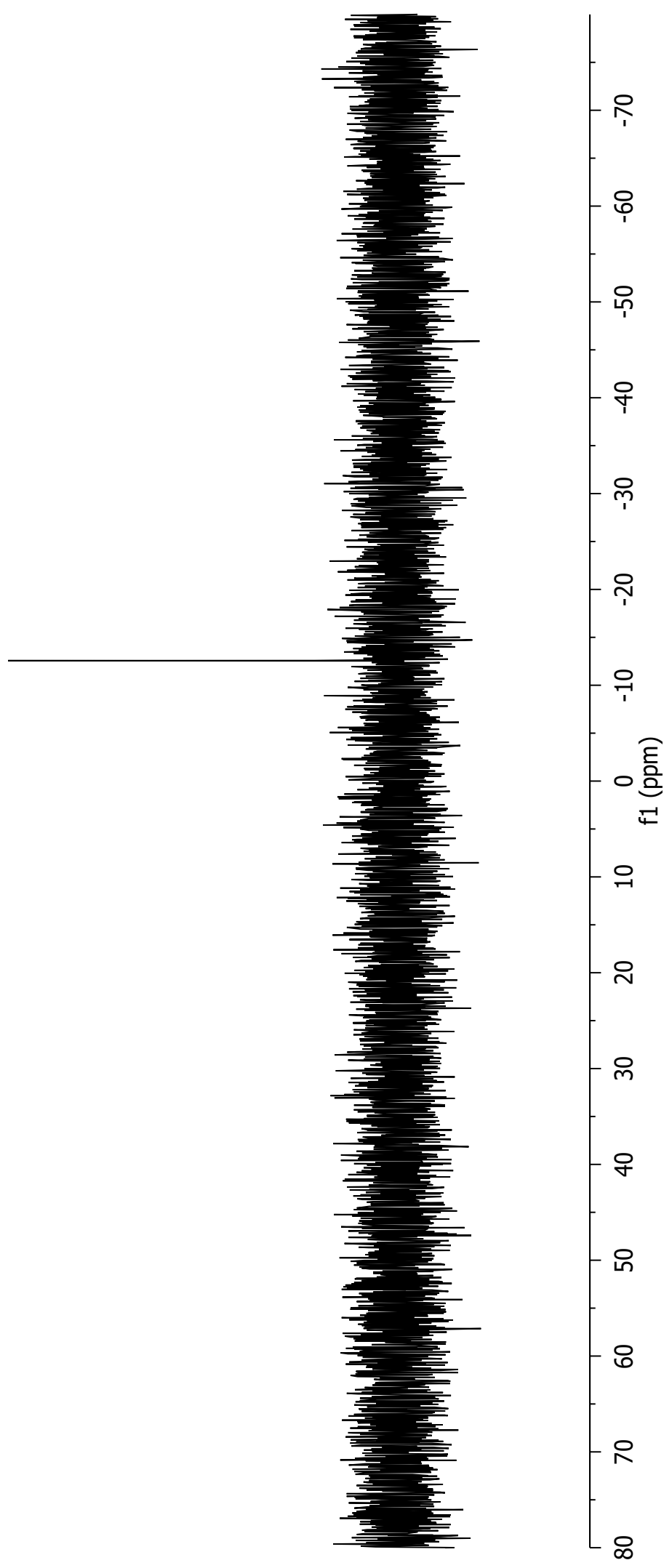


$\angle b^{\circ} 0^{-}$

$\angle 9^{\circ} 0$
$89^{\circ} 0^{\prime}$

6L'I

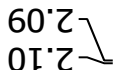

sદ'Z

$9 \varepsilon ' Z \succ$

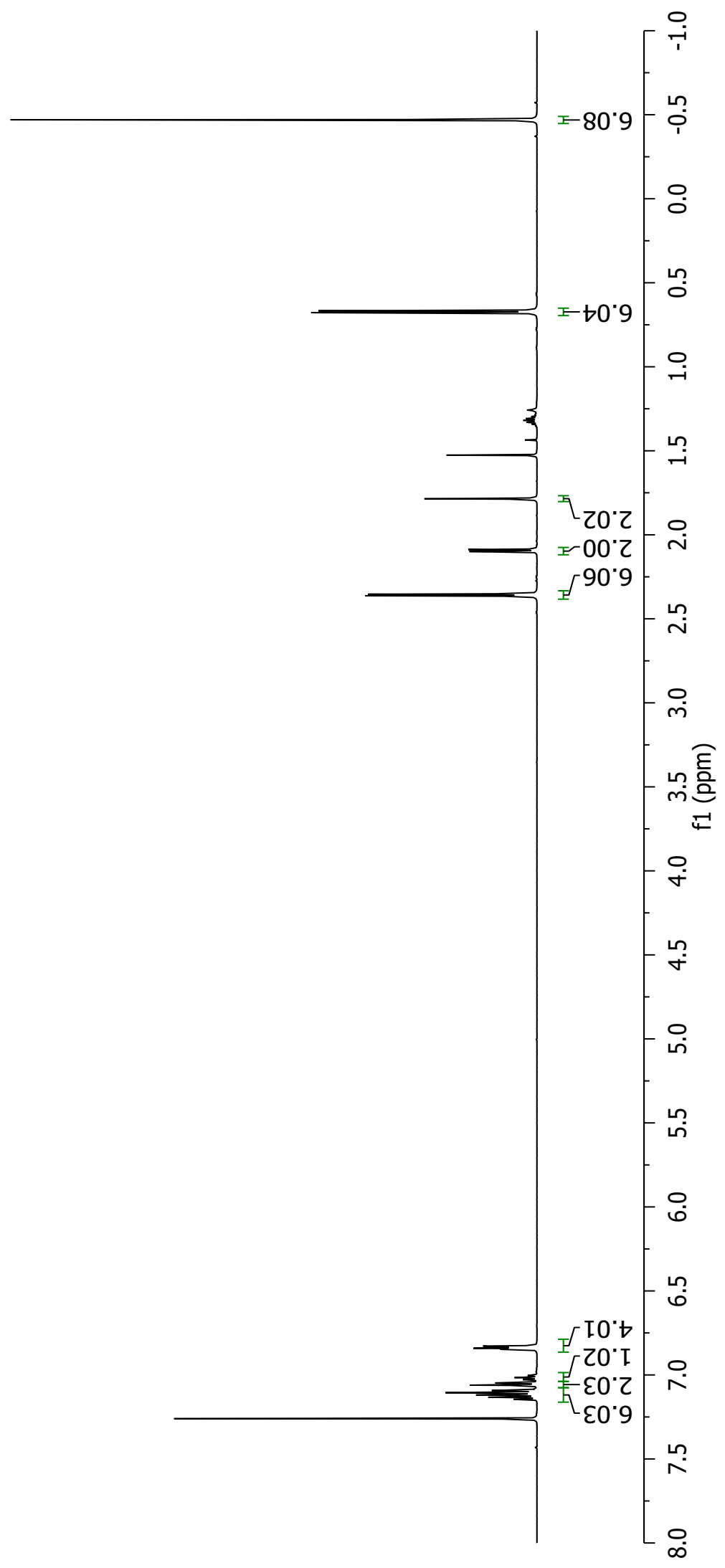




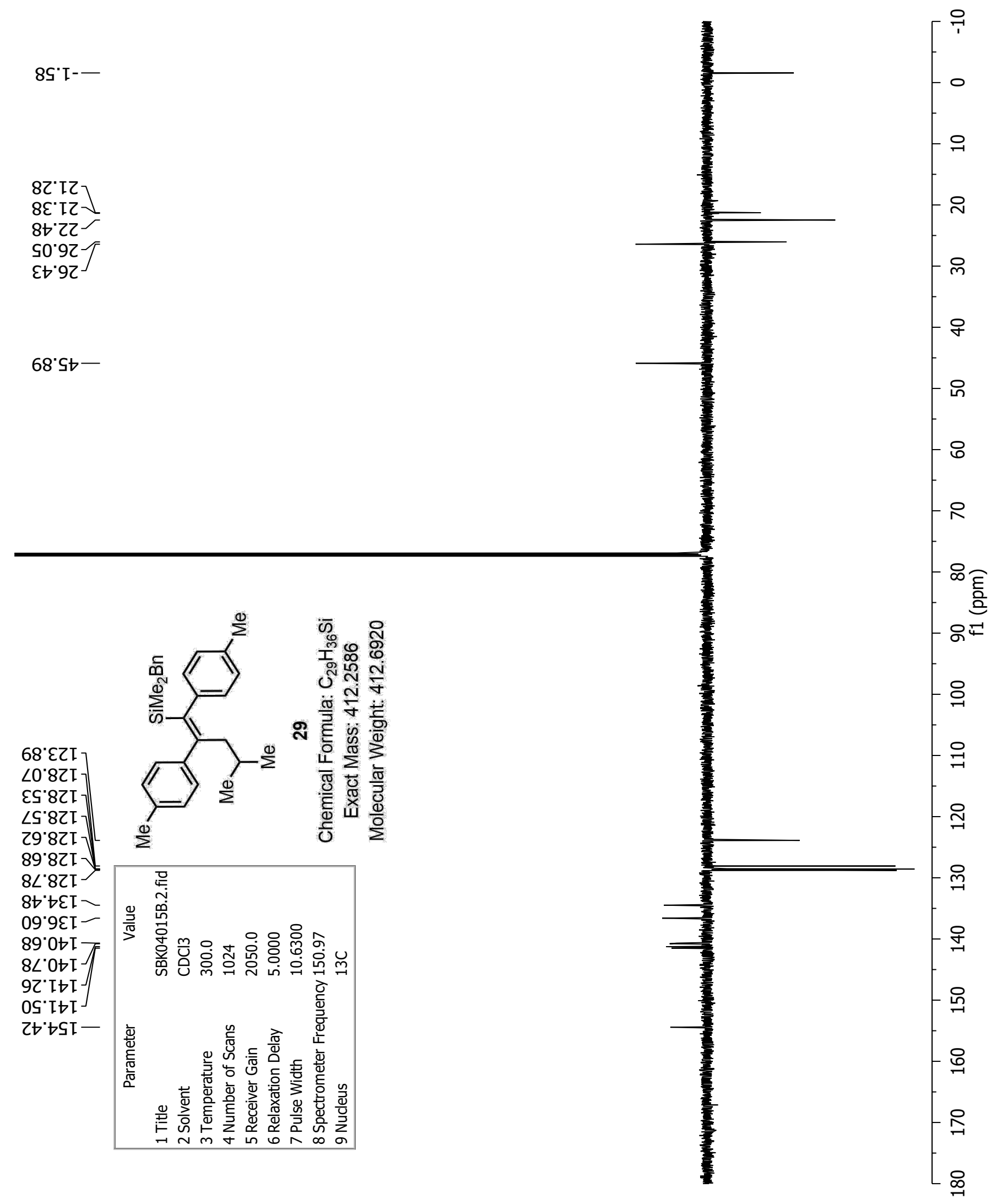




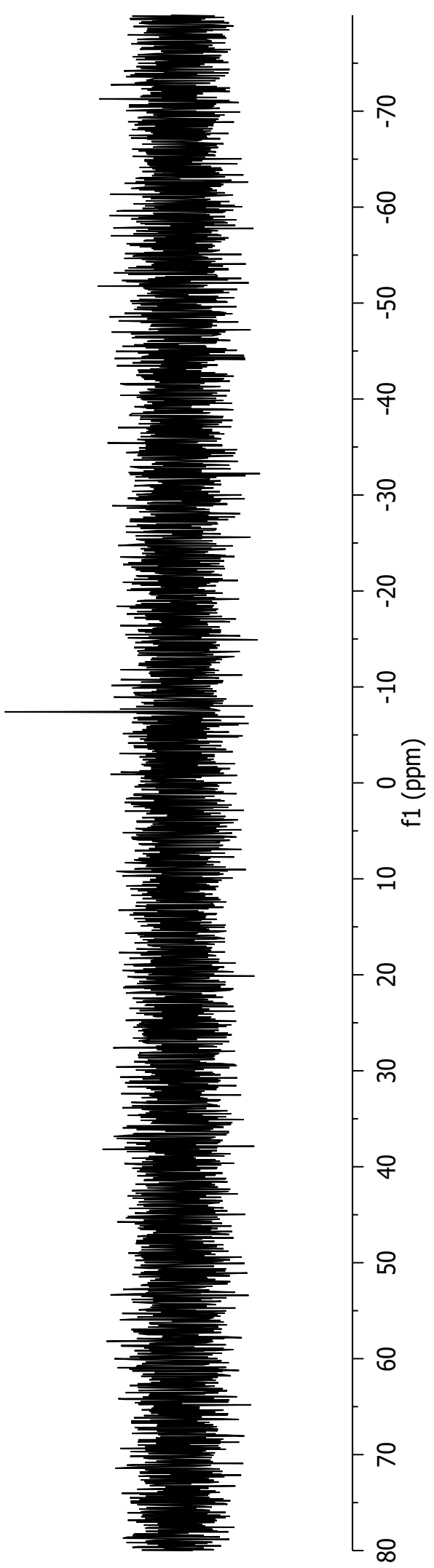


$8 t^{\circ} \varepsilon-$
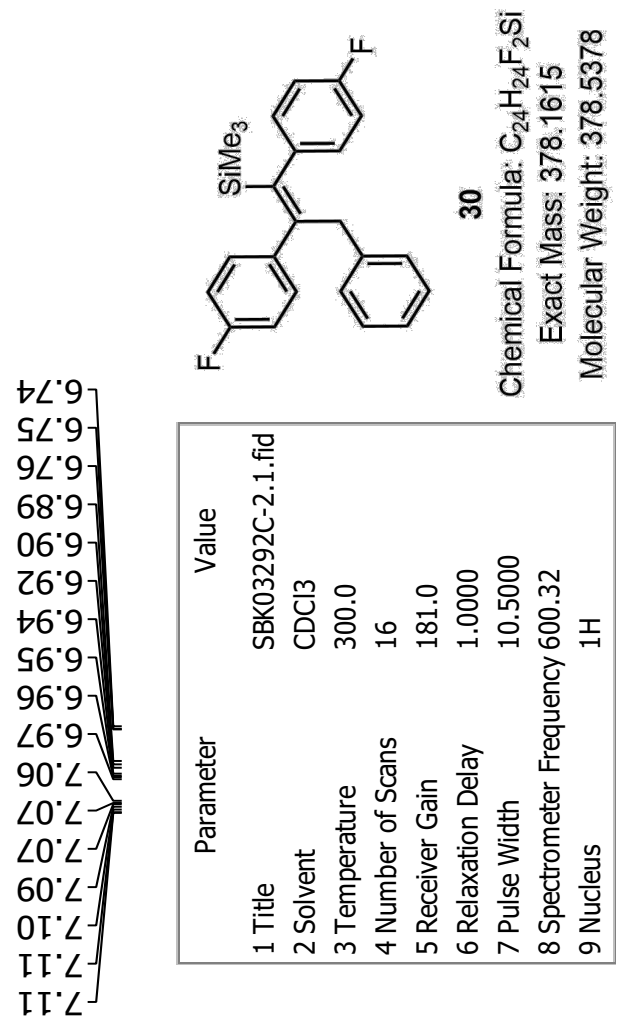

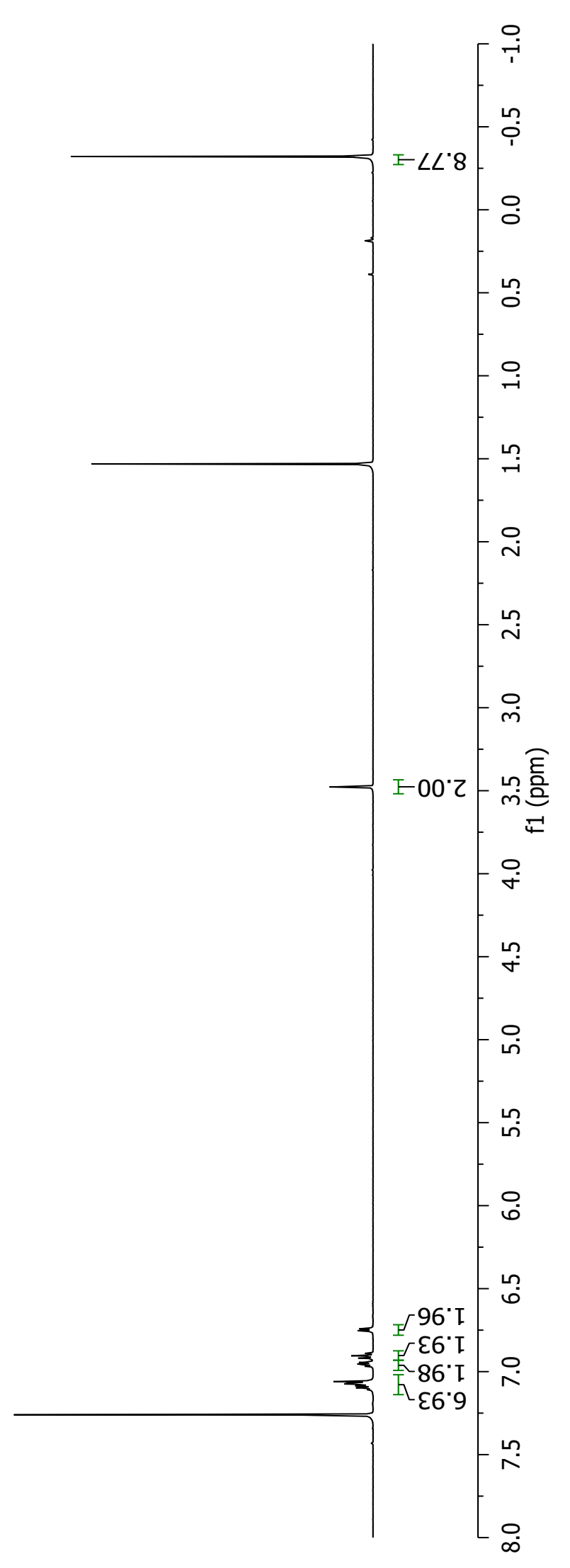



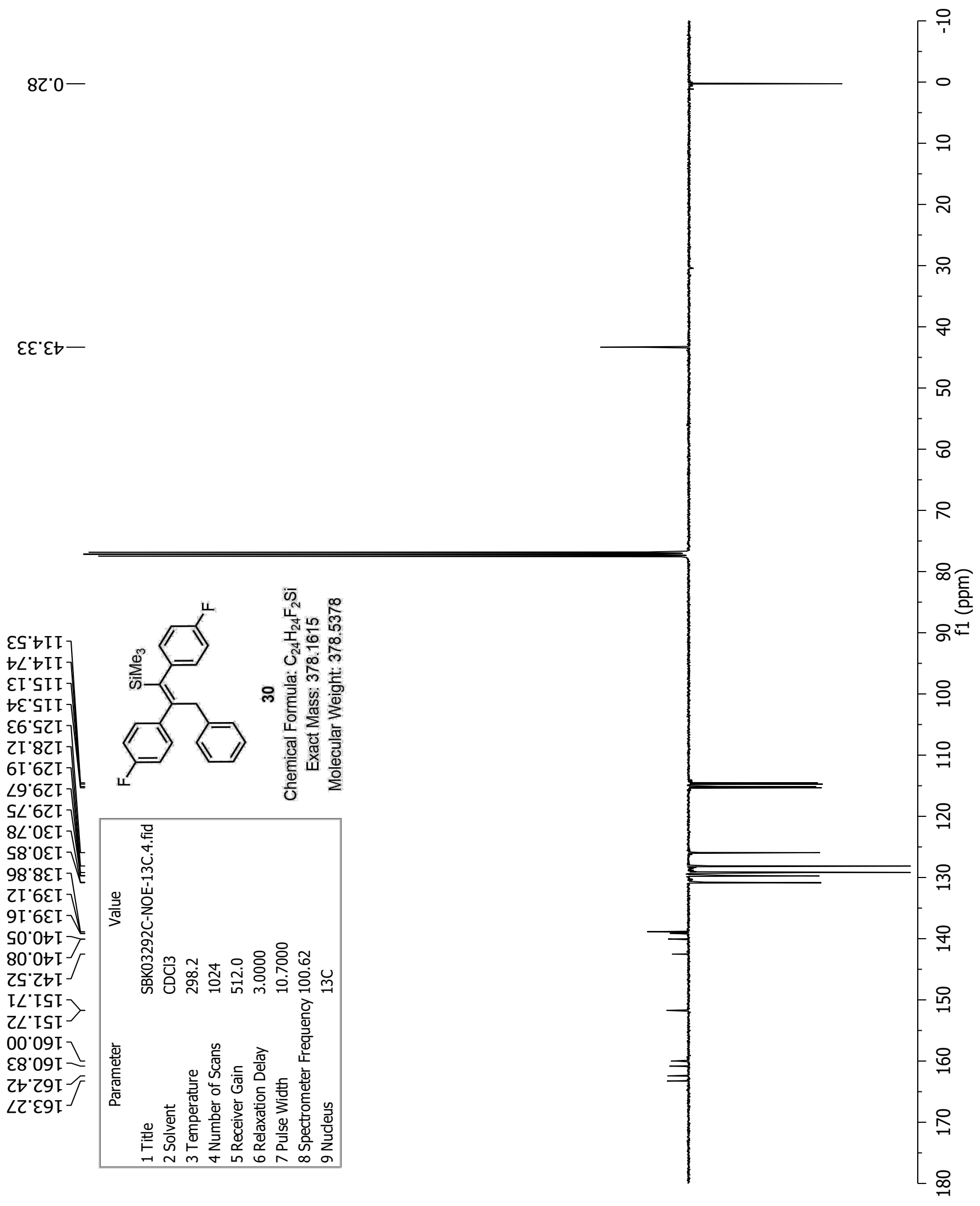

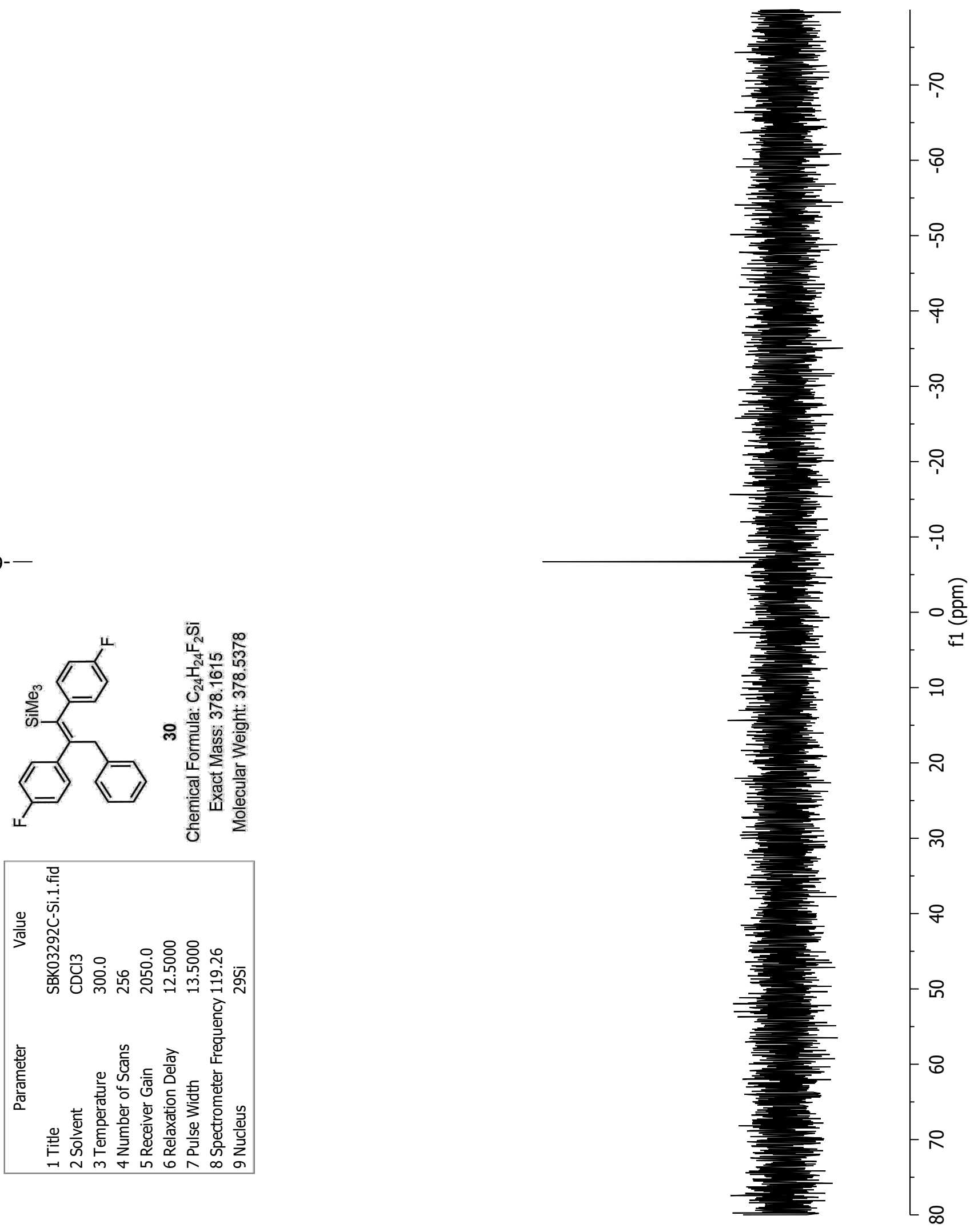

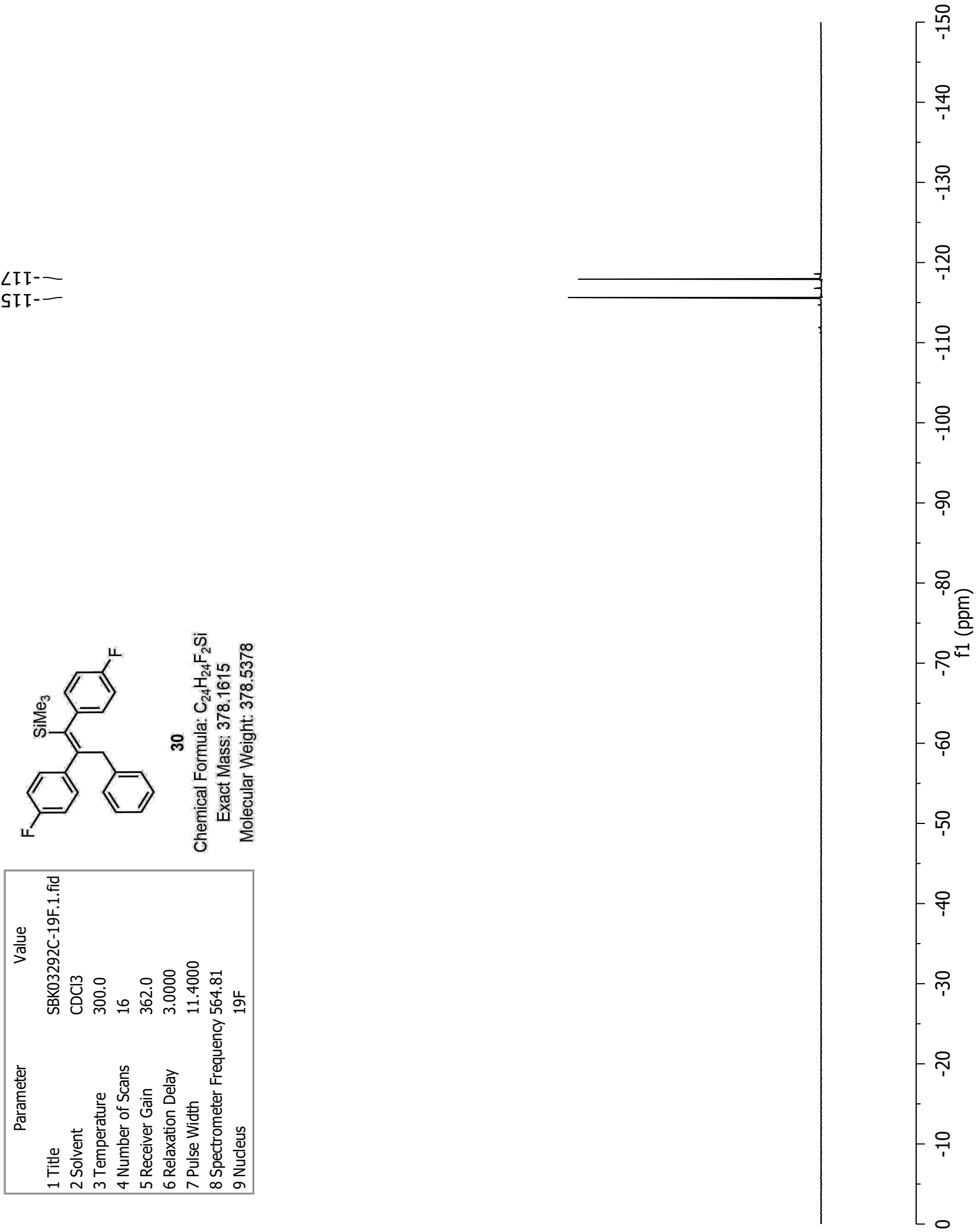

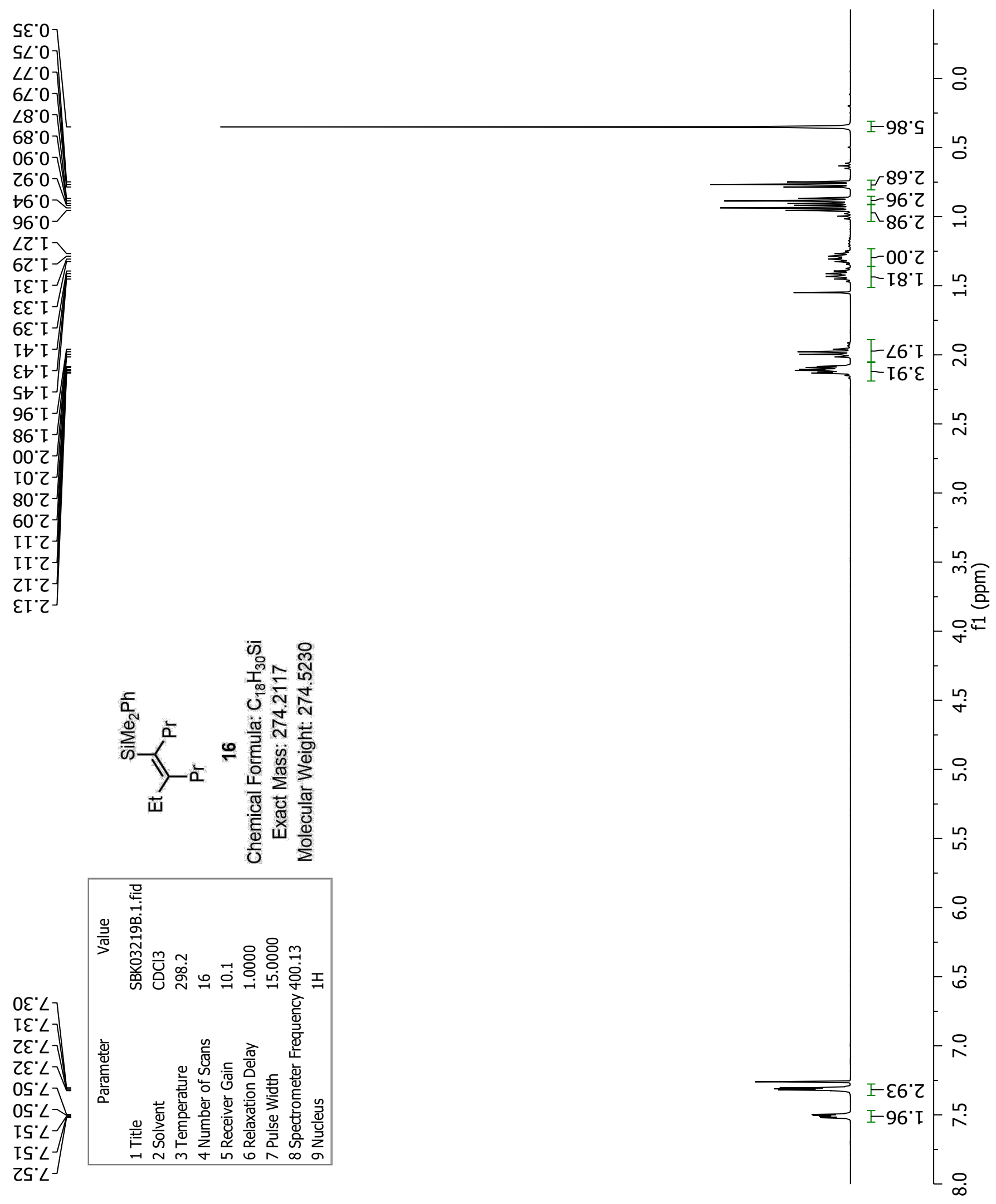


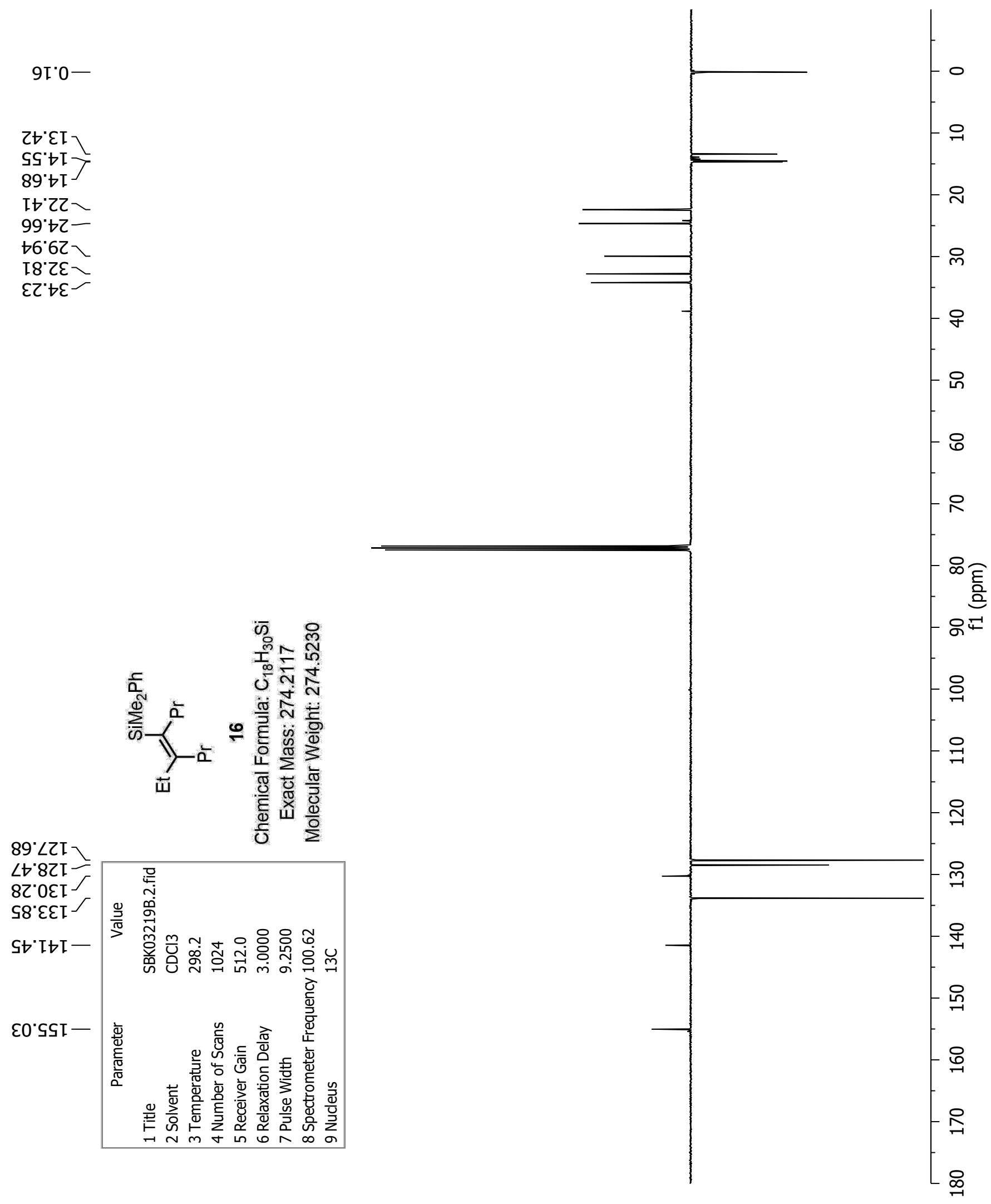


II'II-

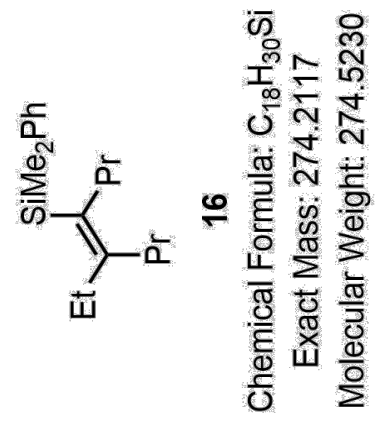

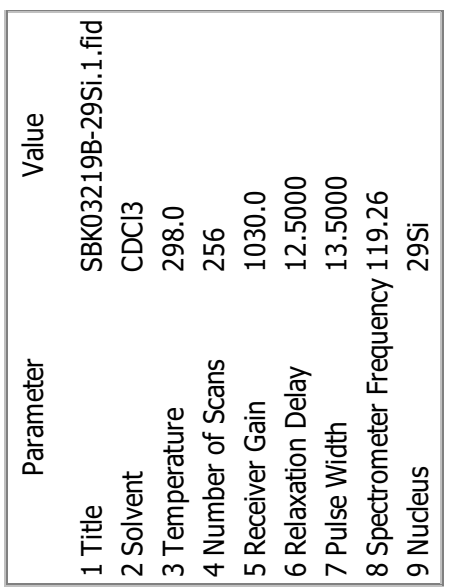

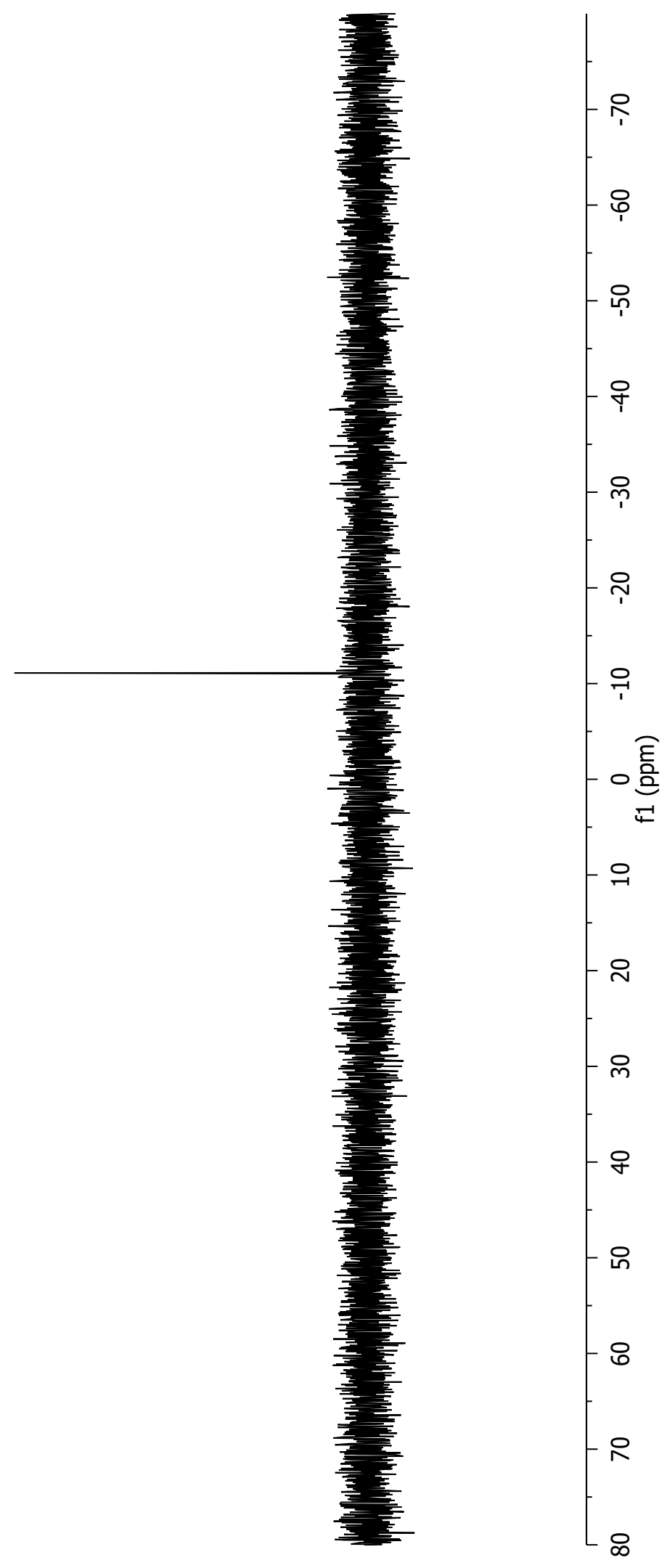




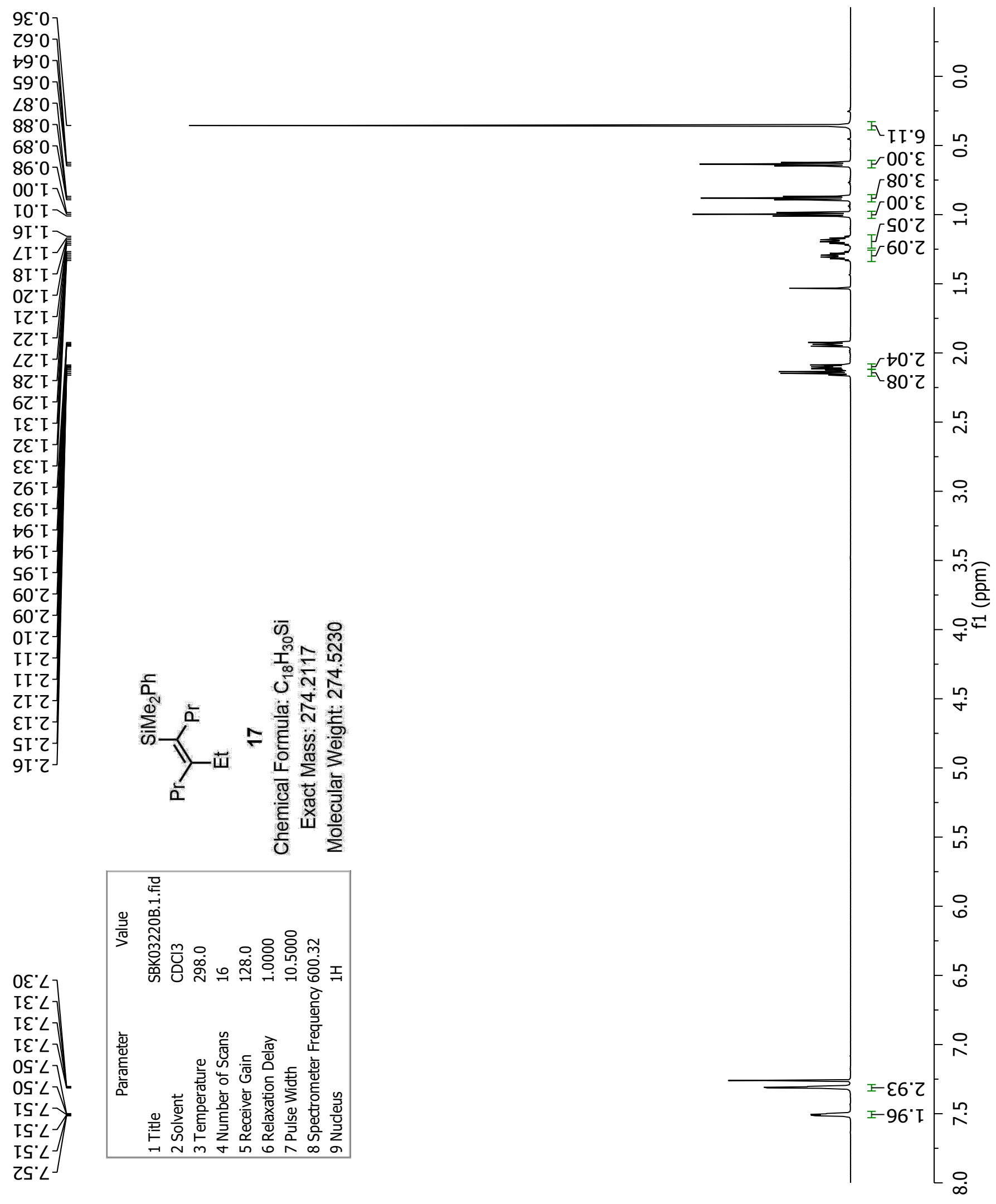




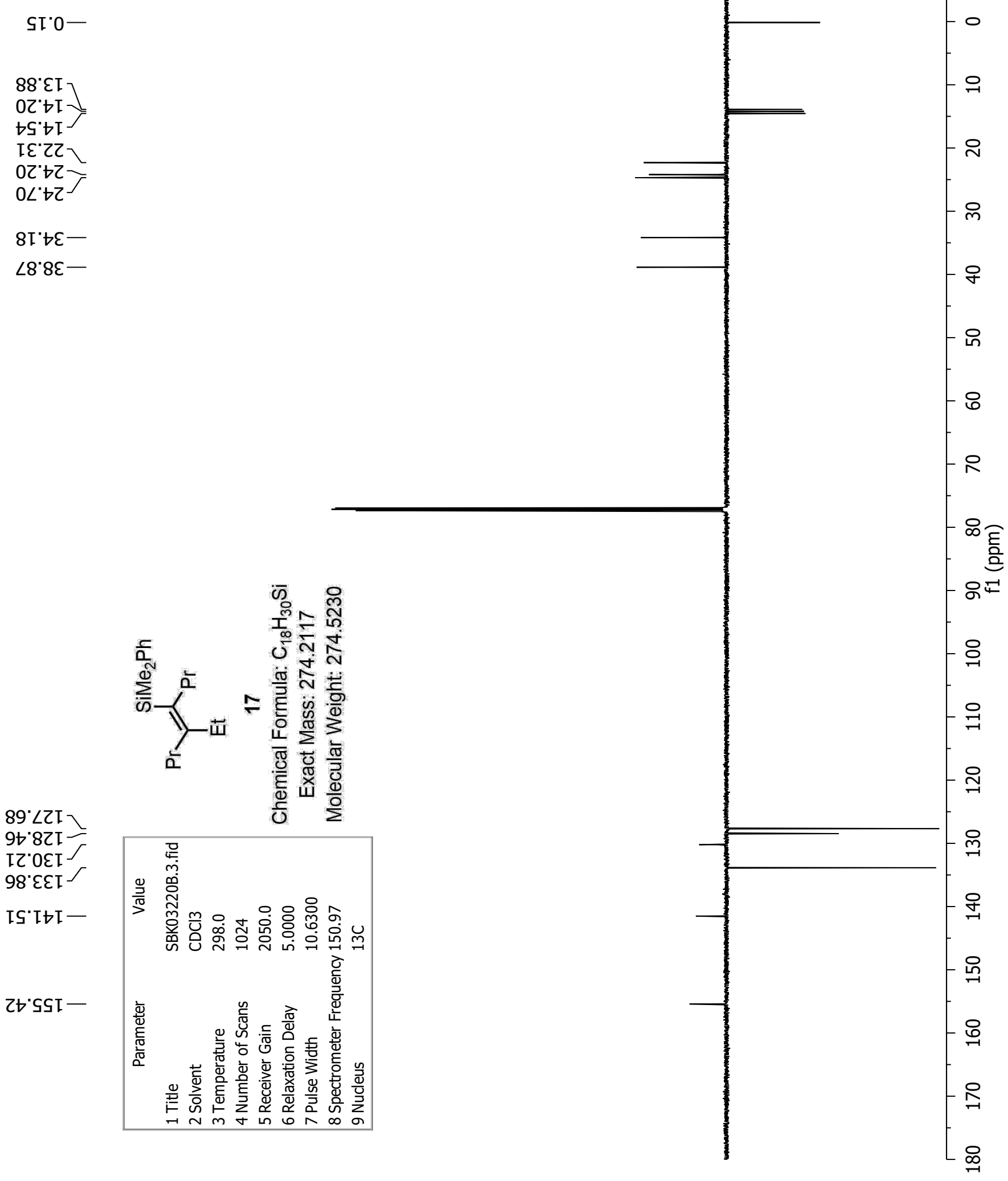



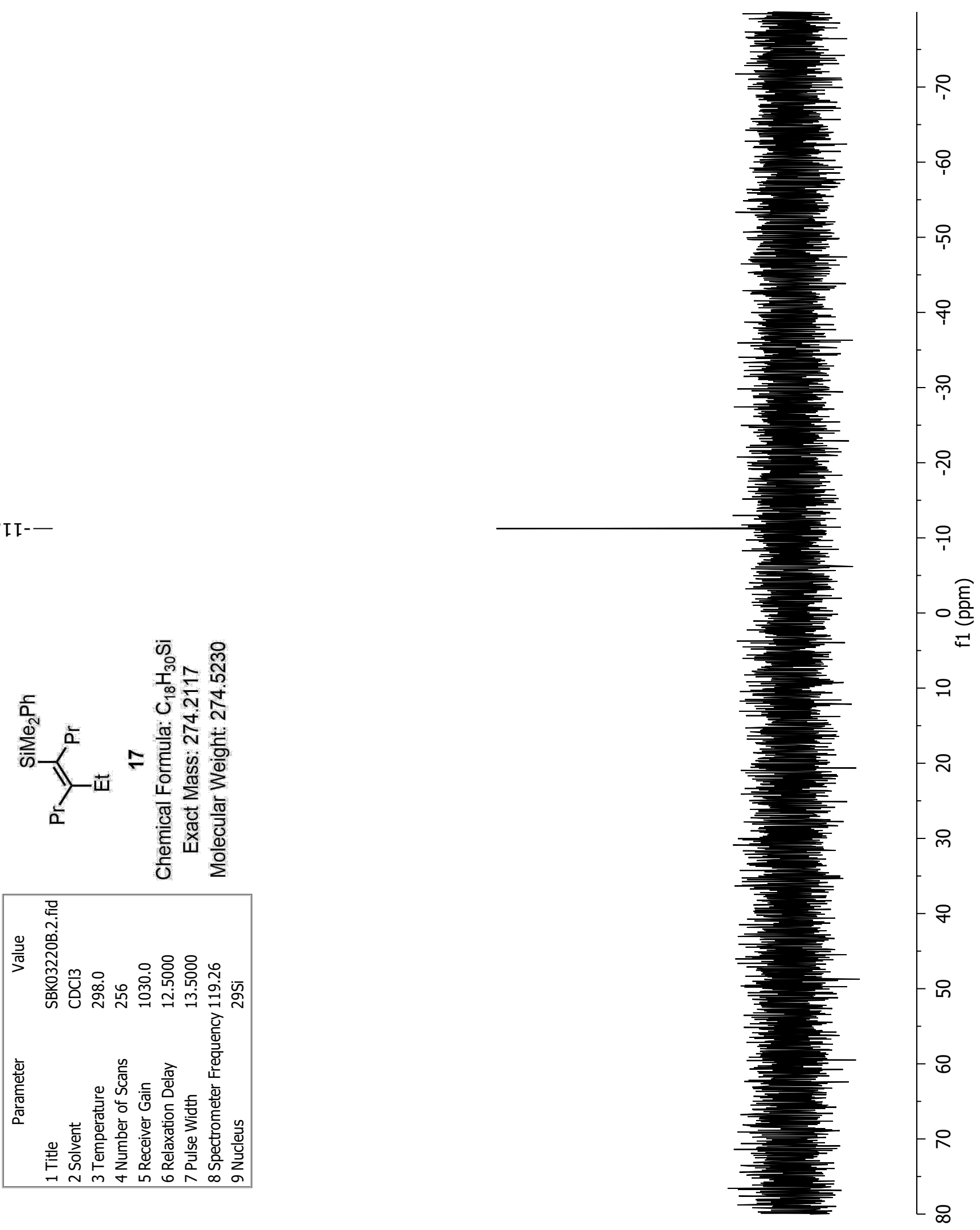


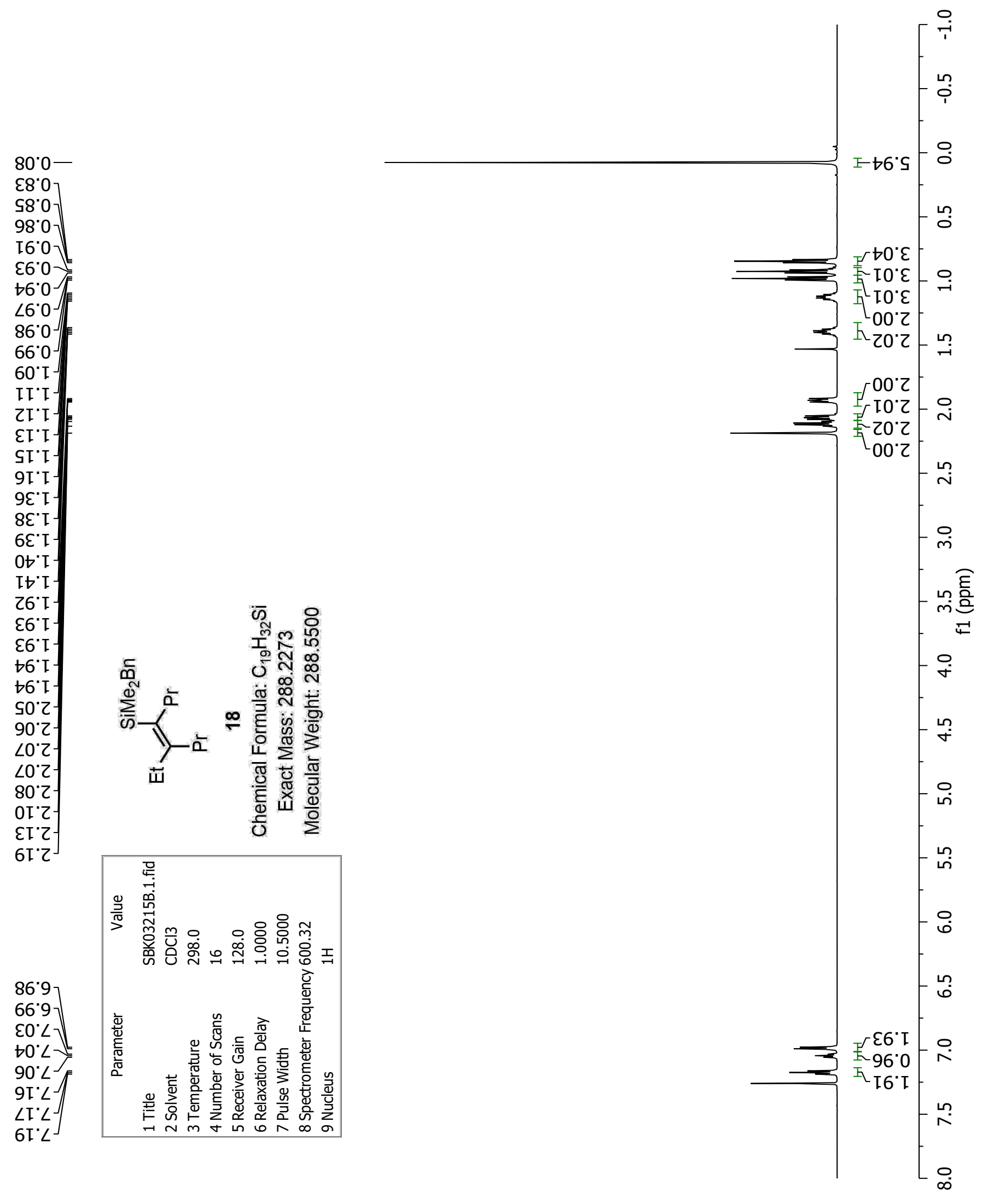



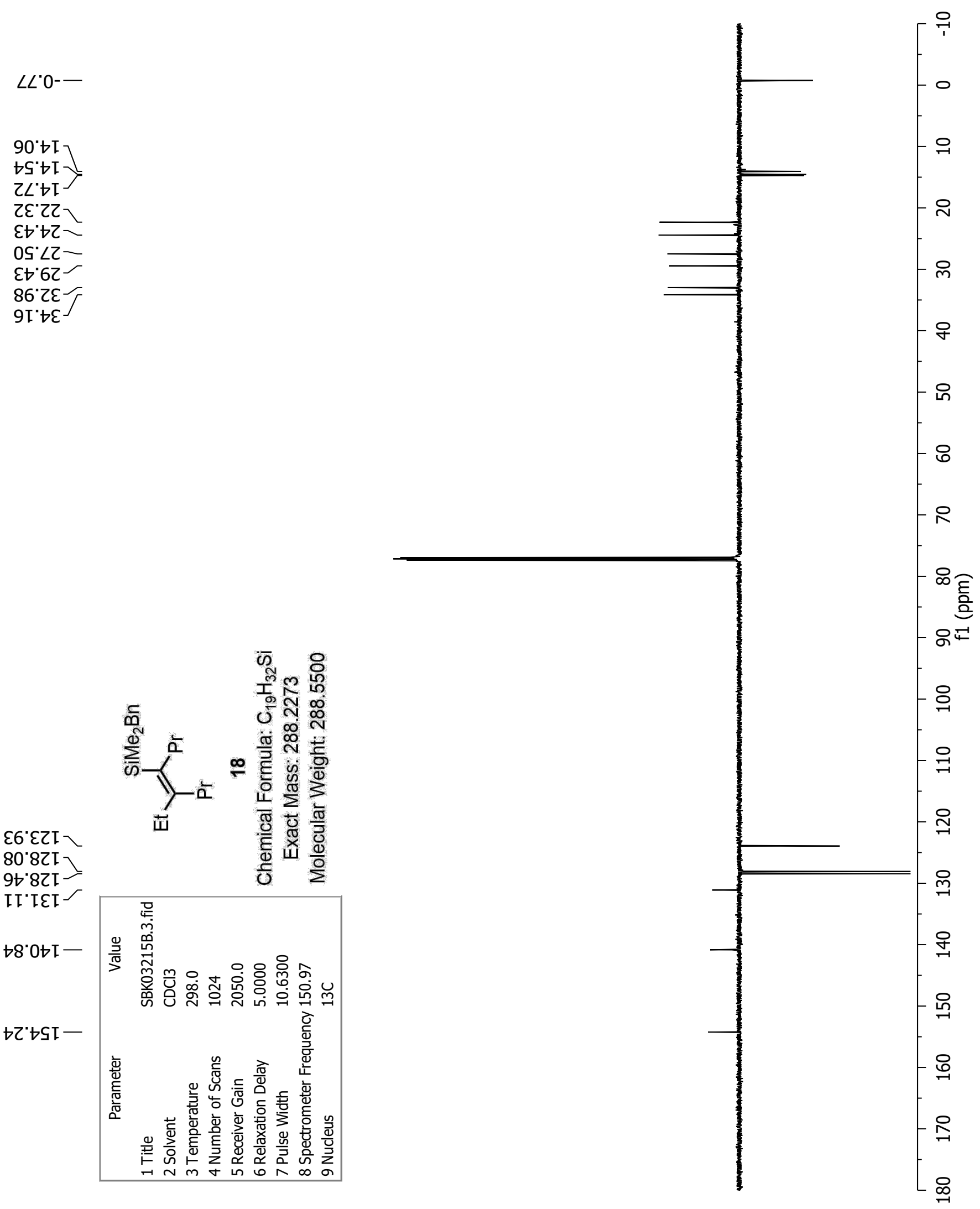

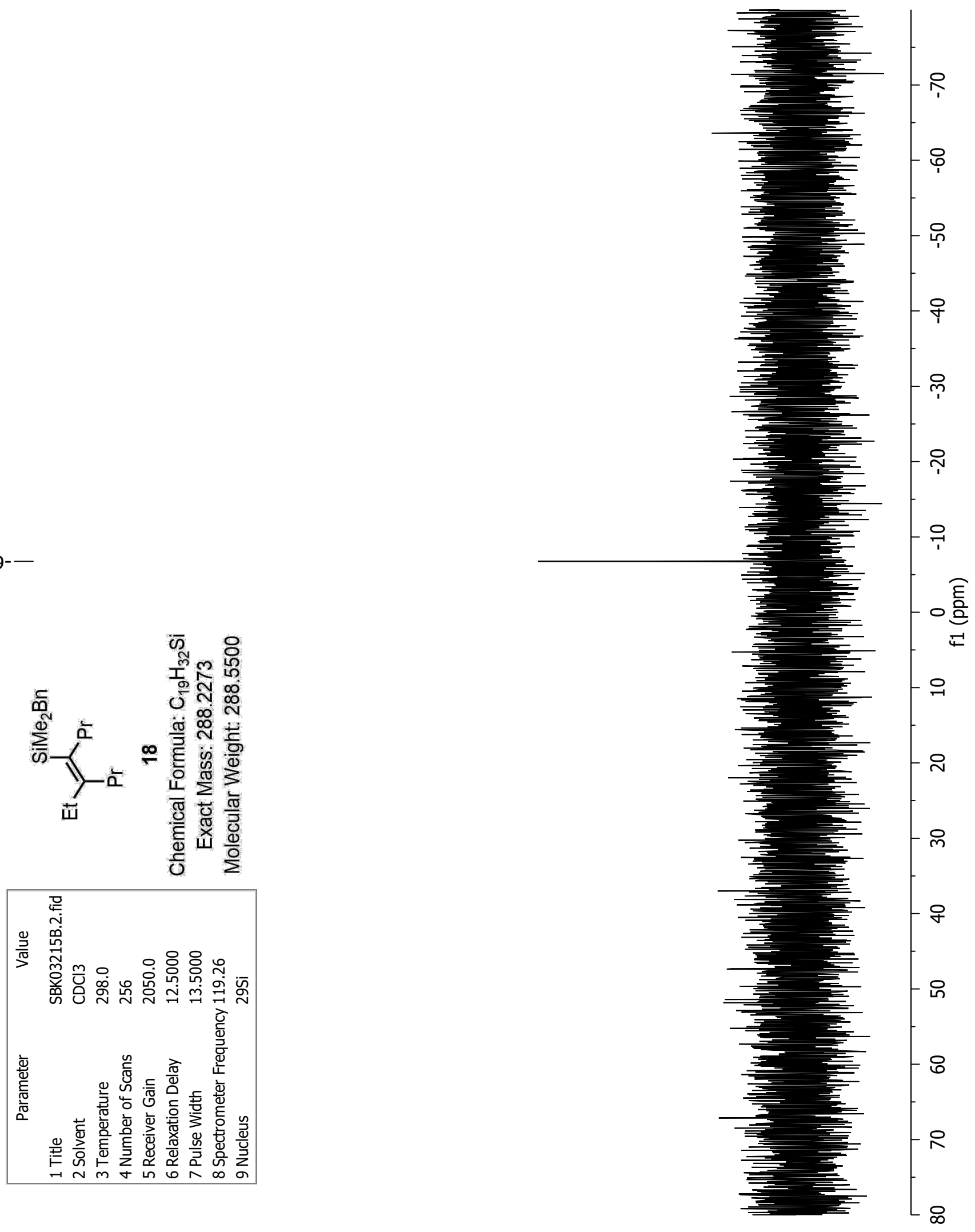


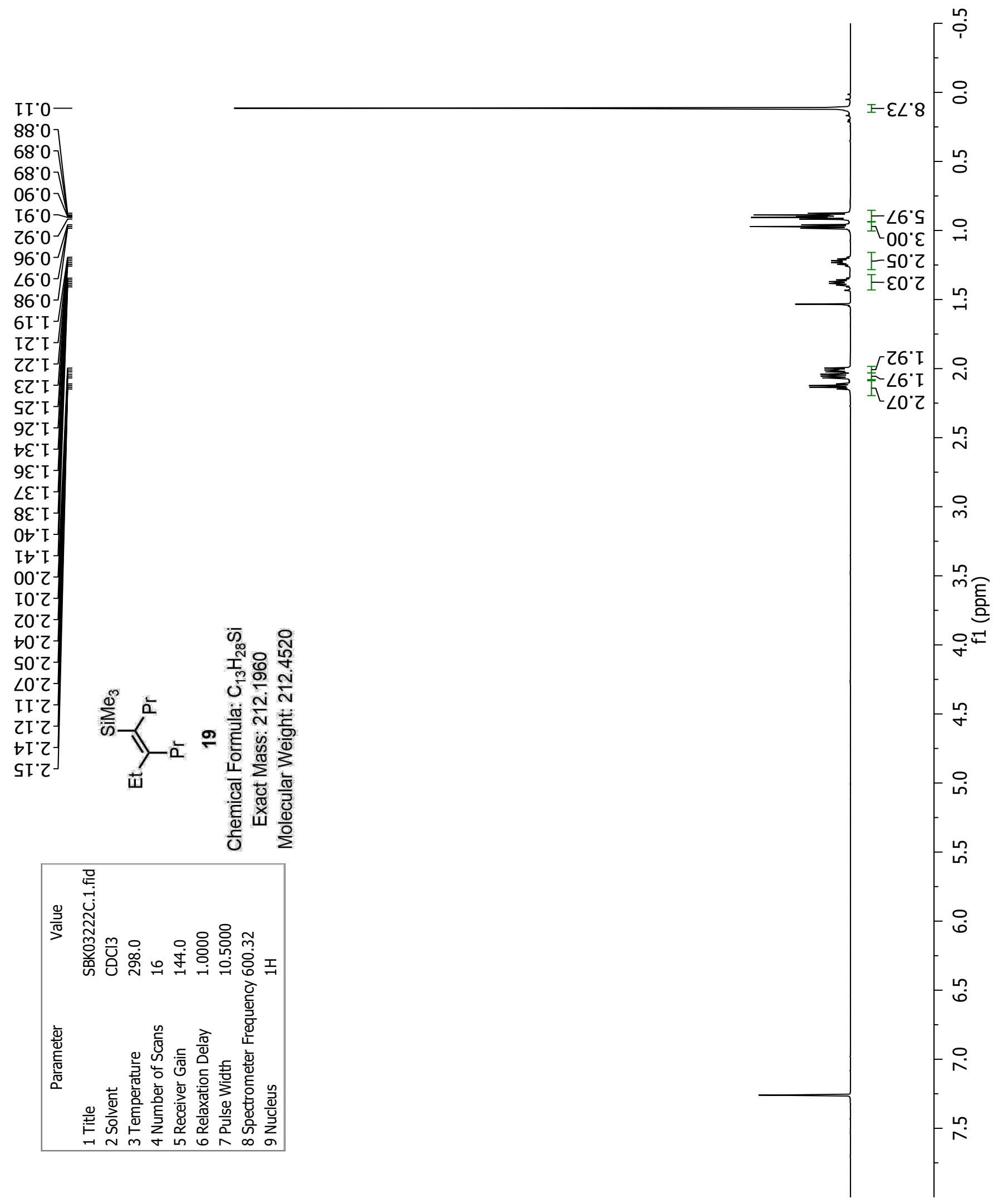



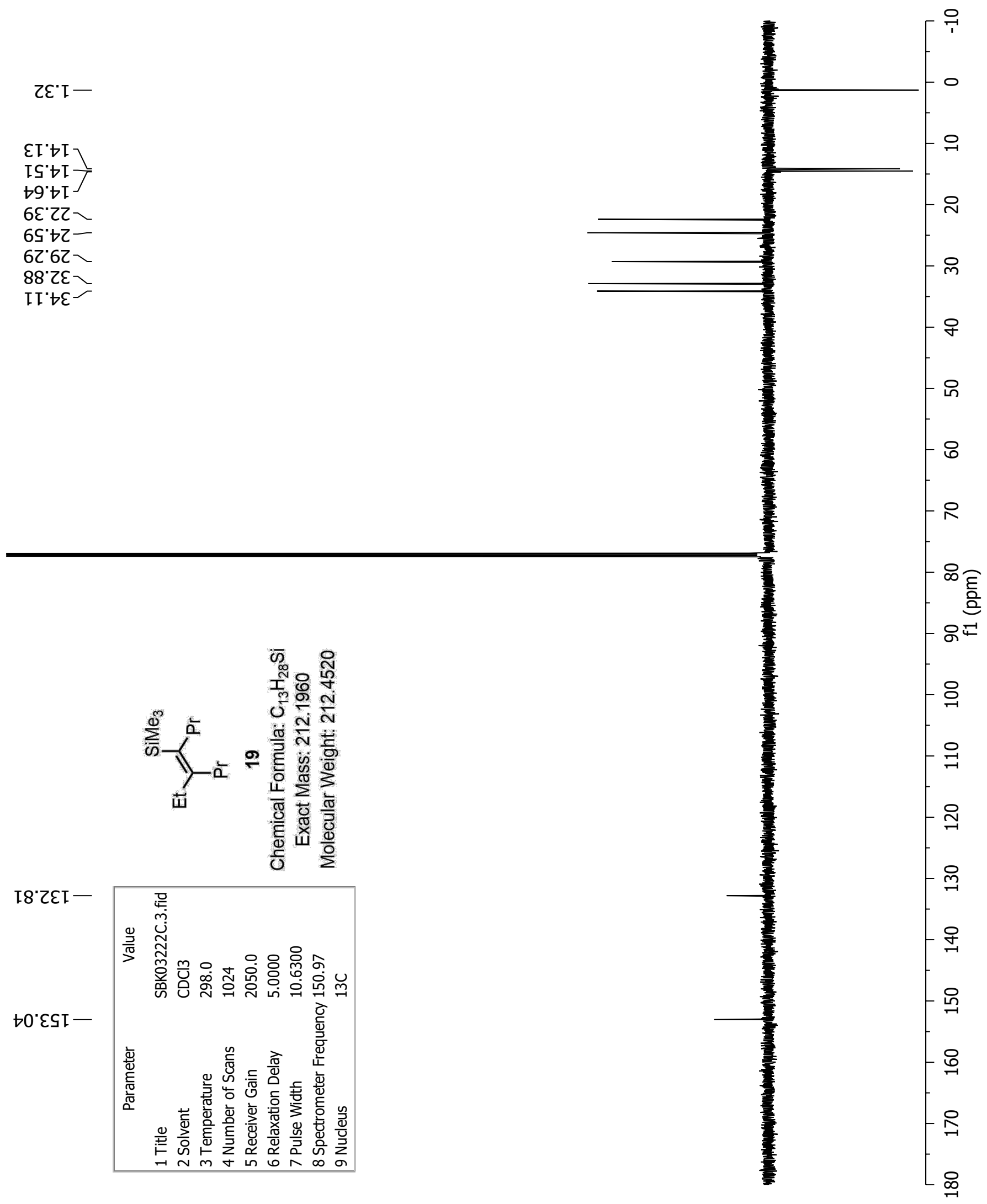


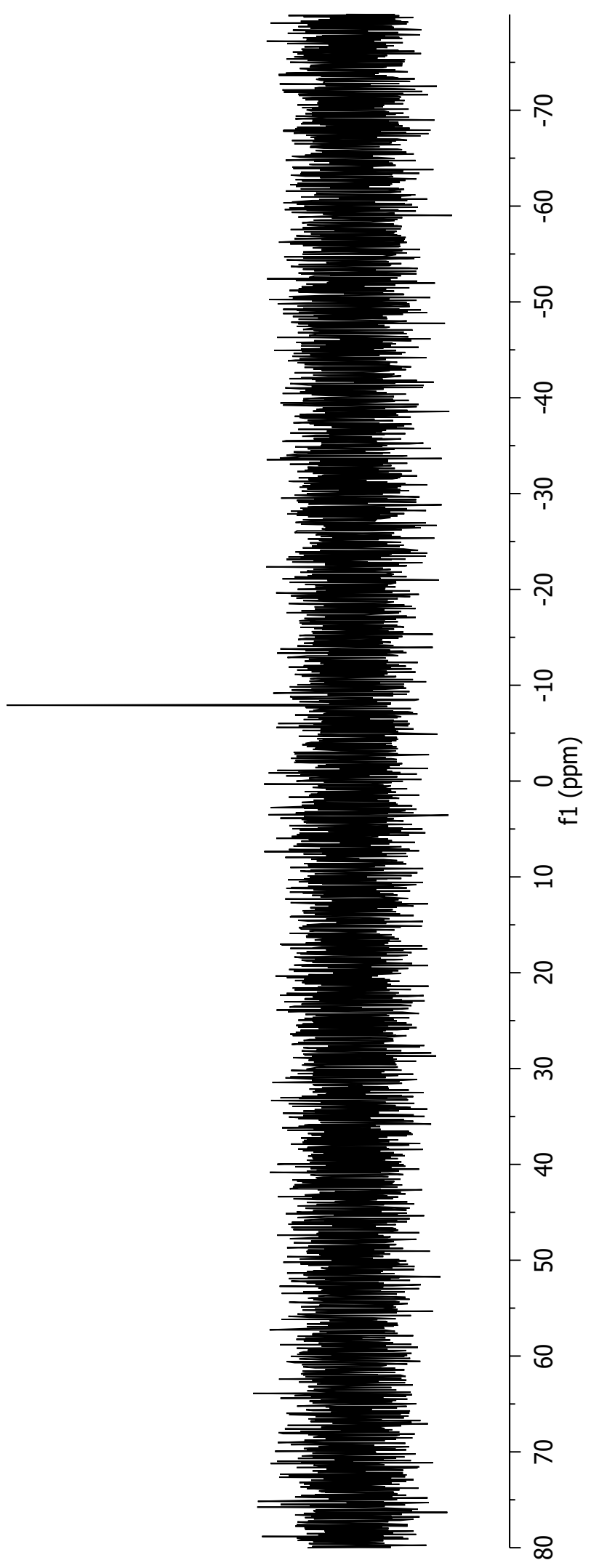



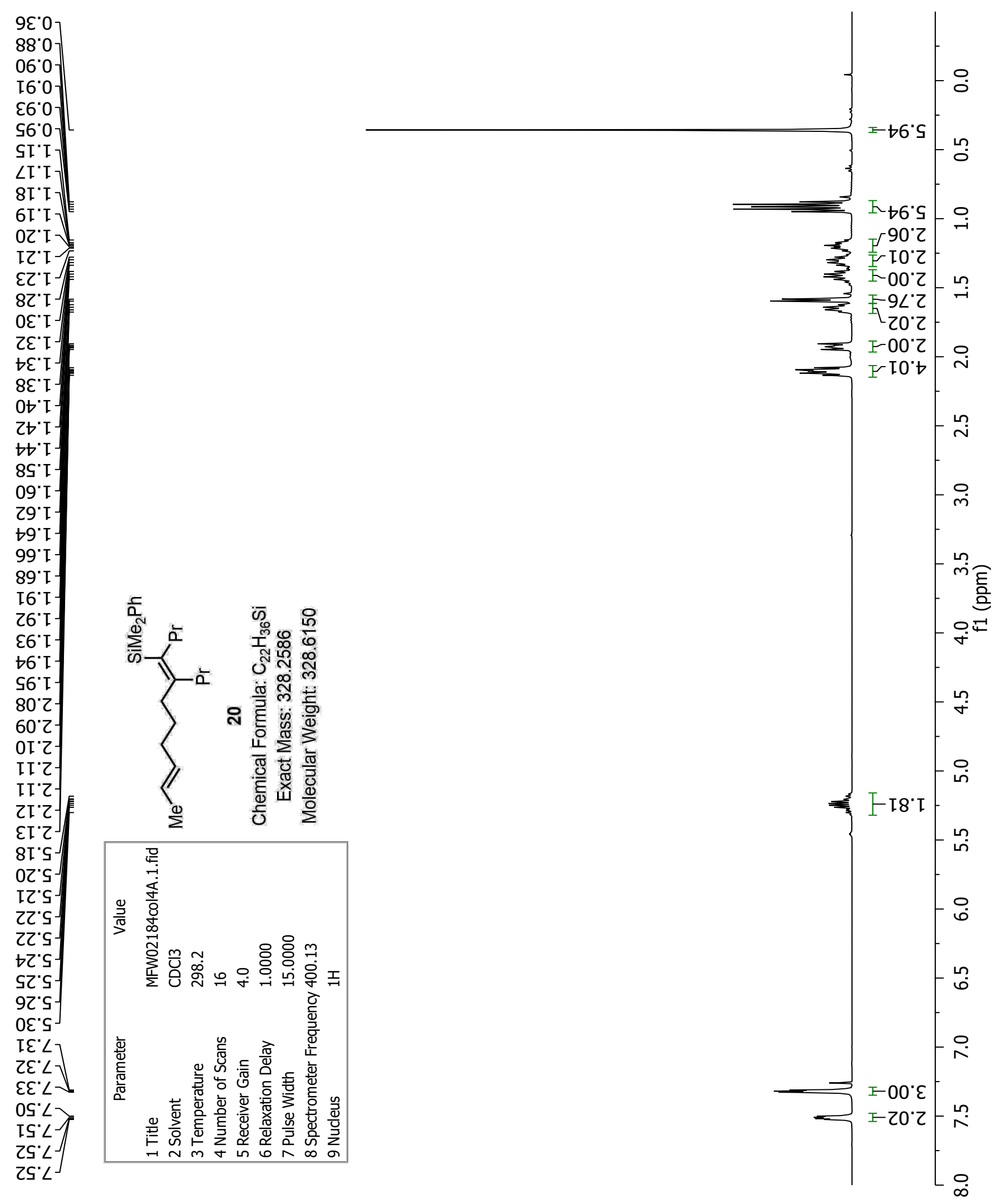

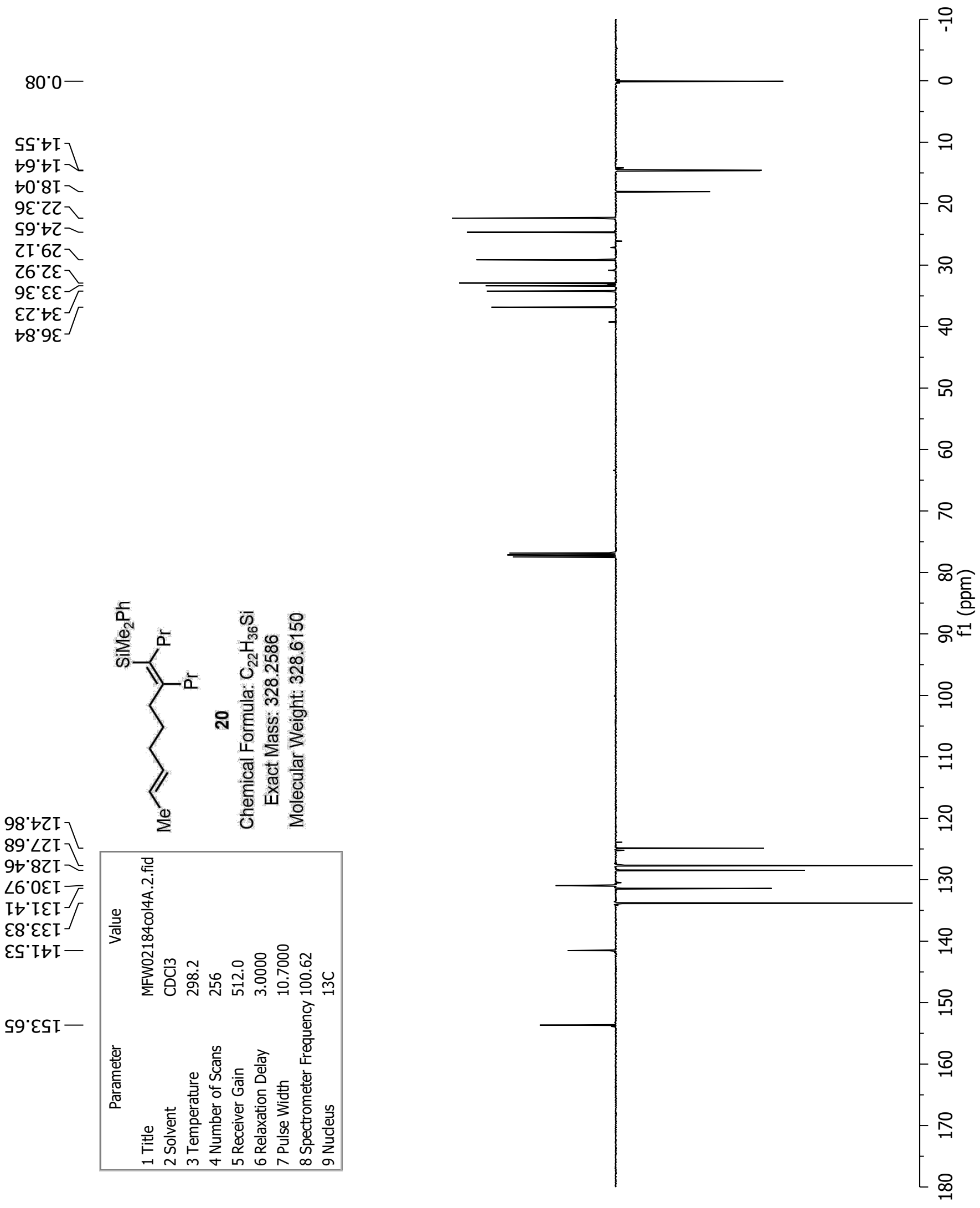

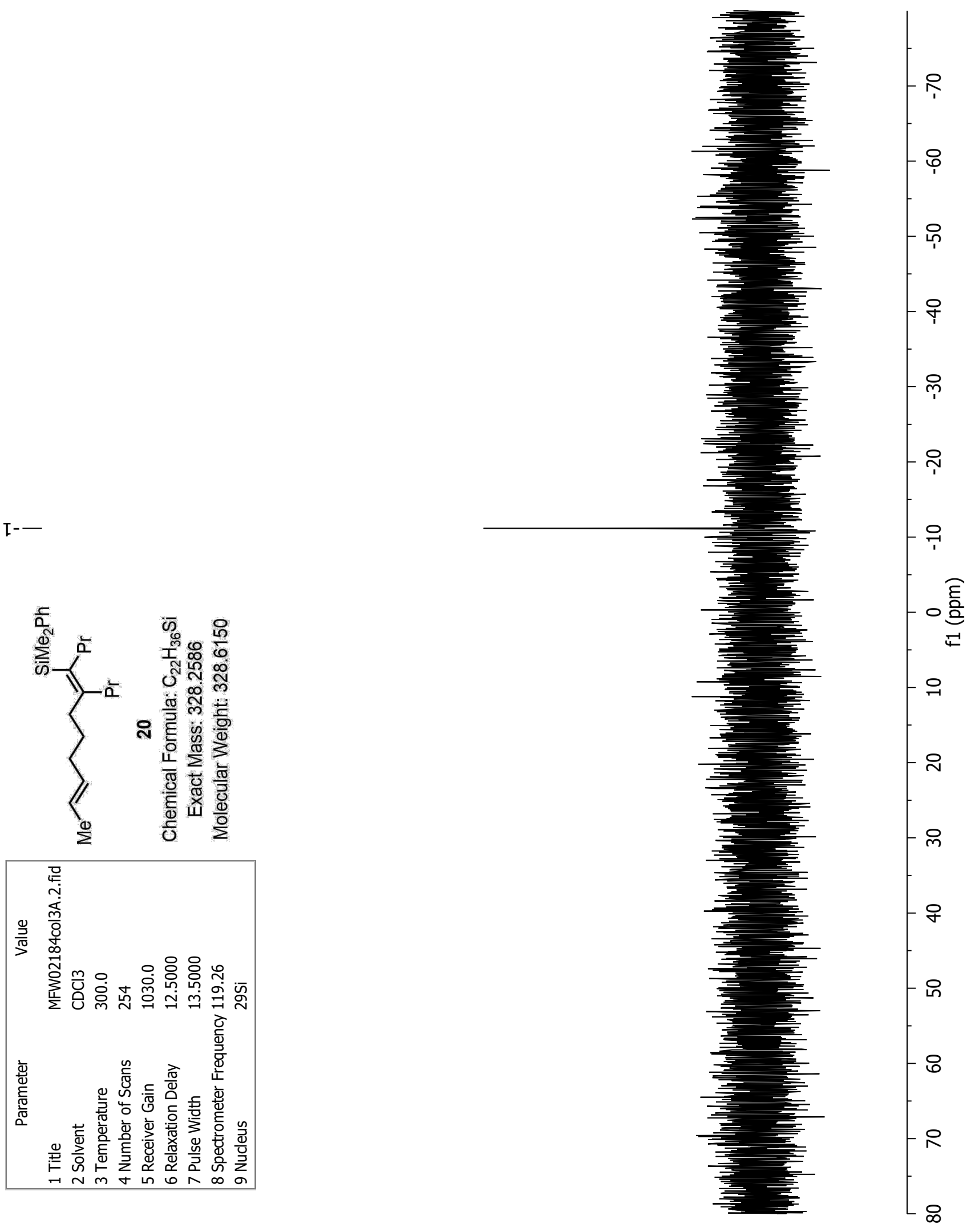


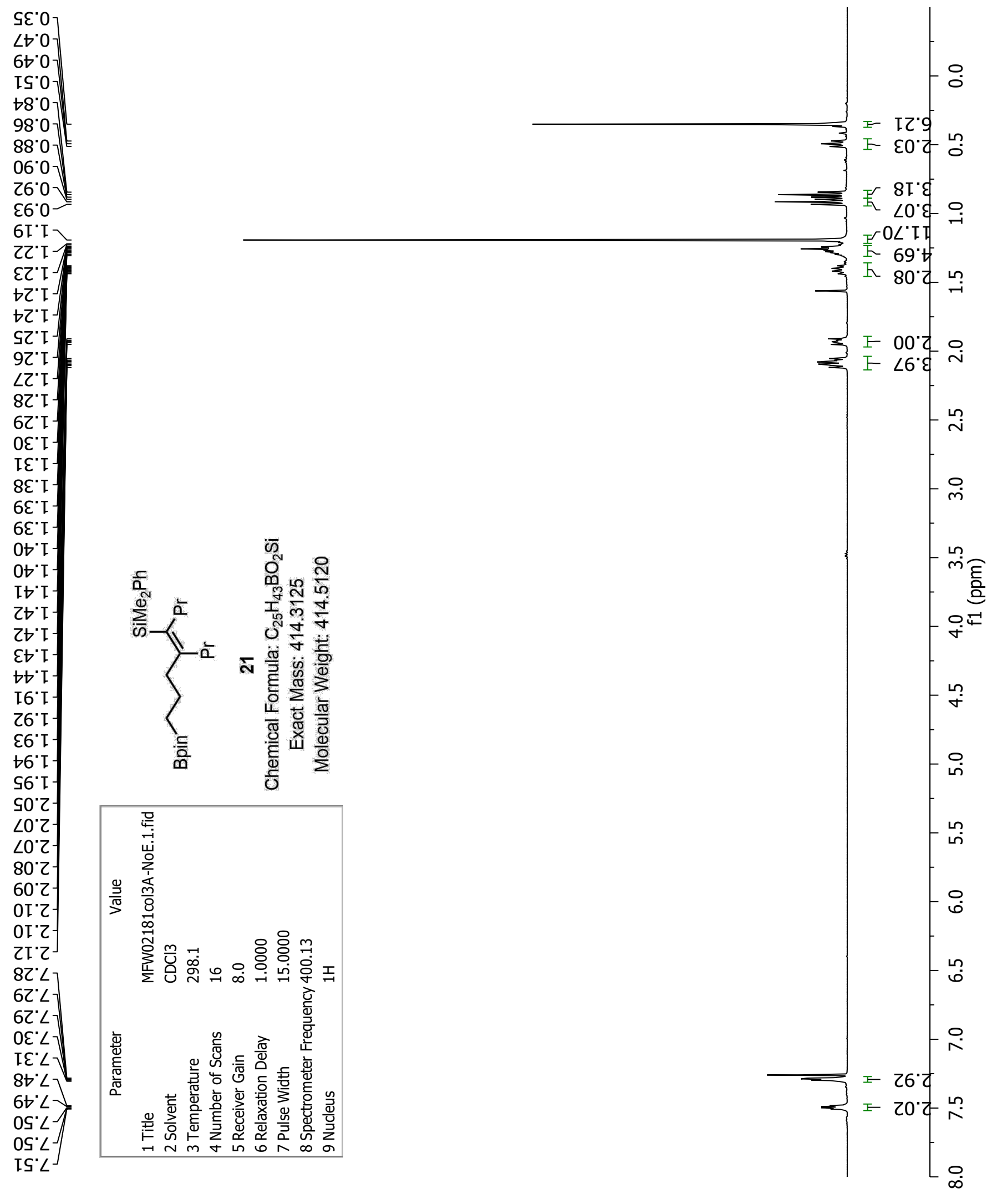




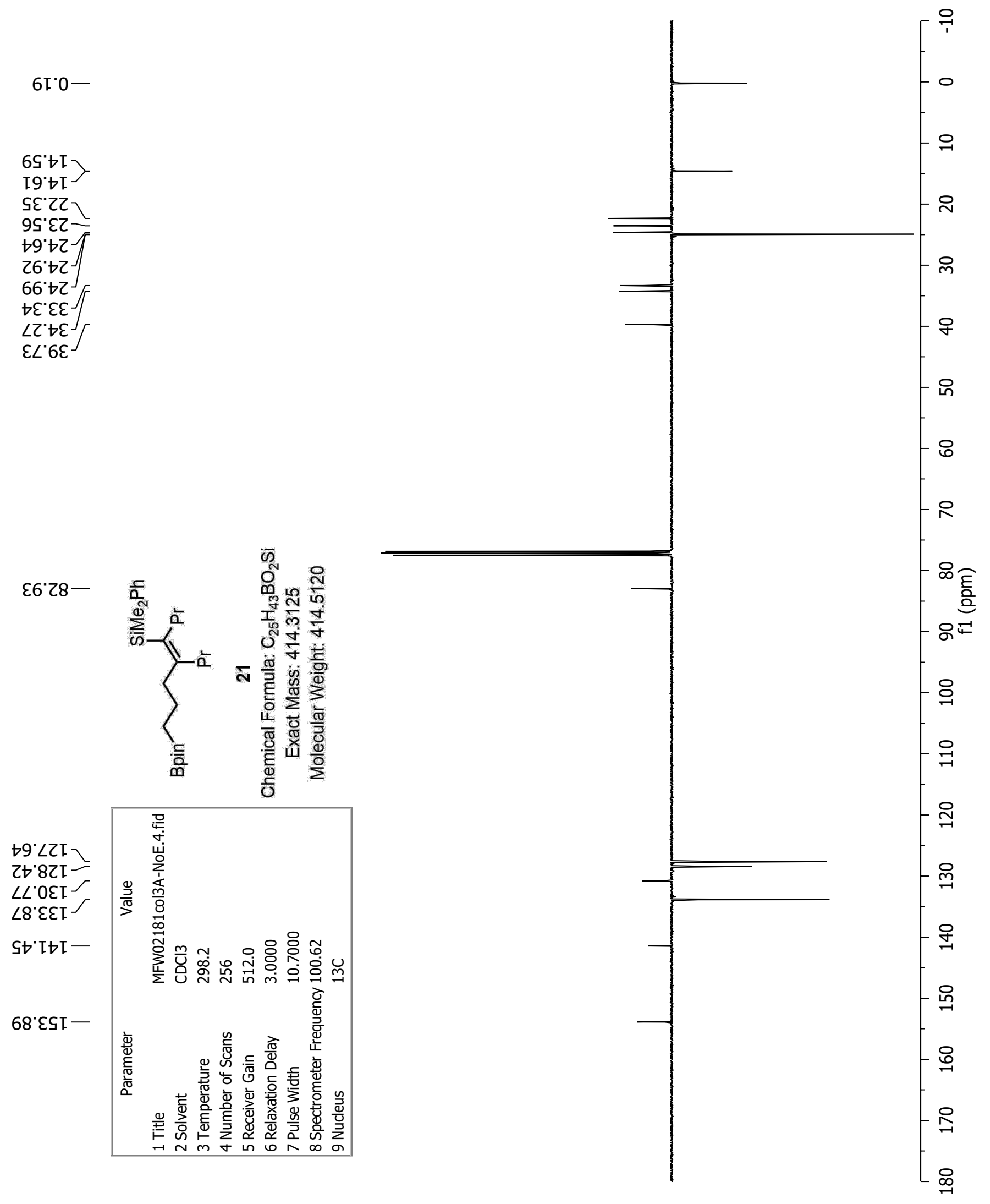


IZ'II-
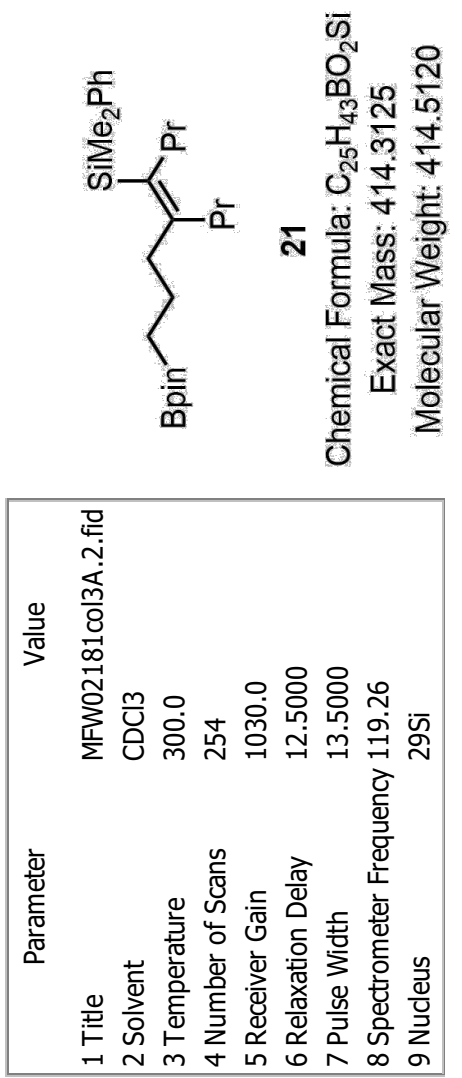

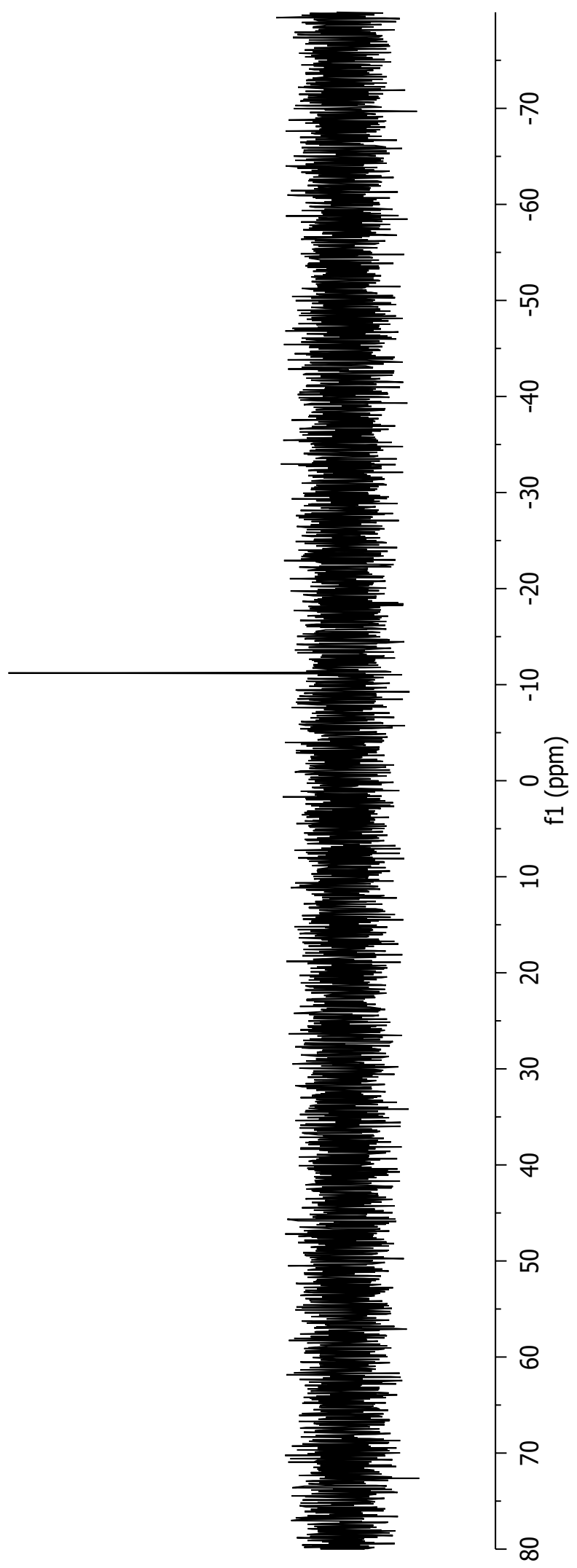



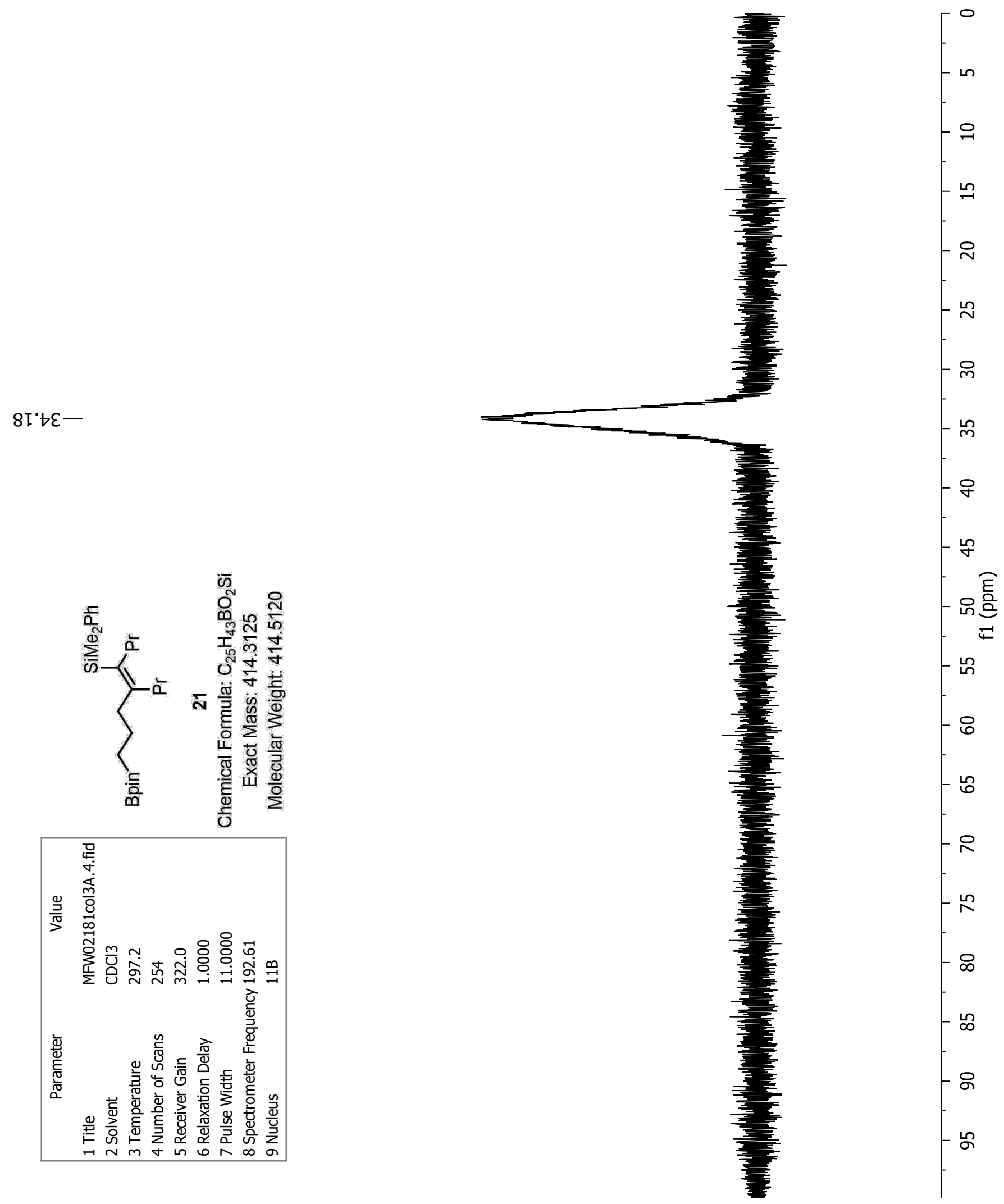


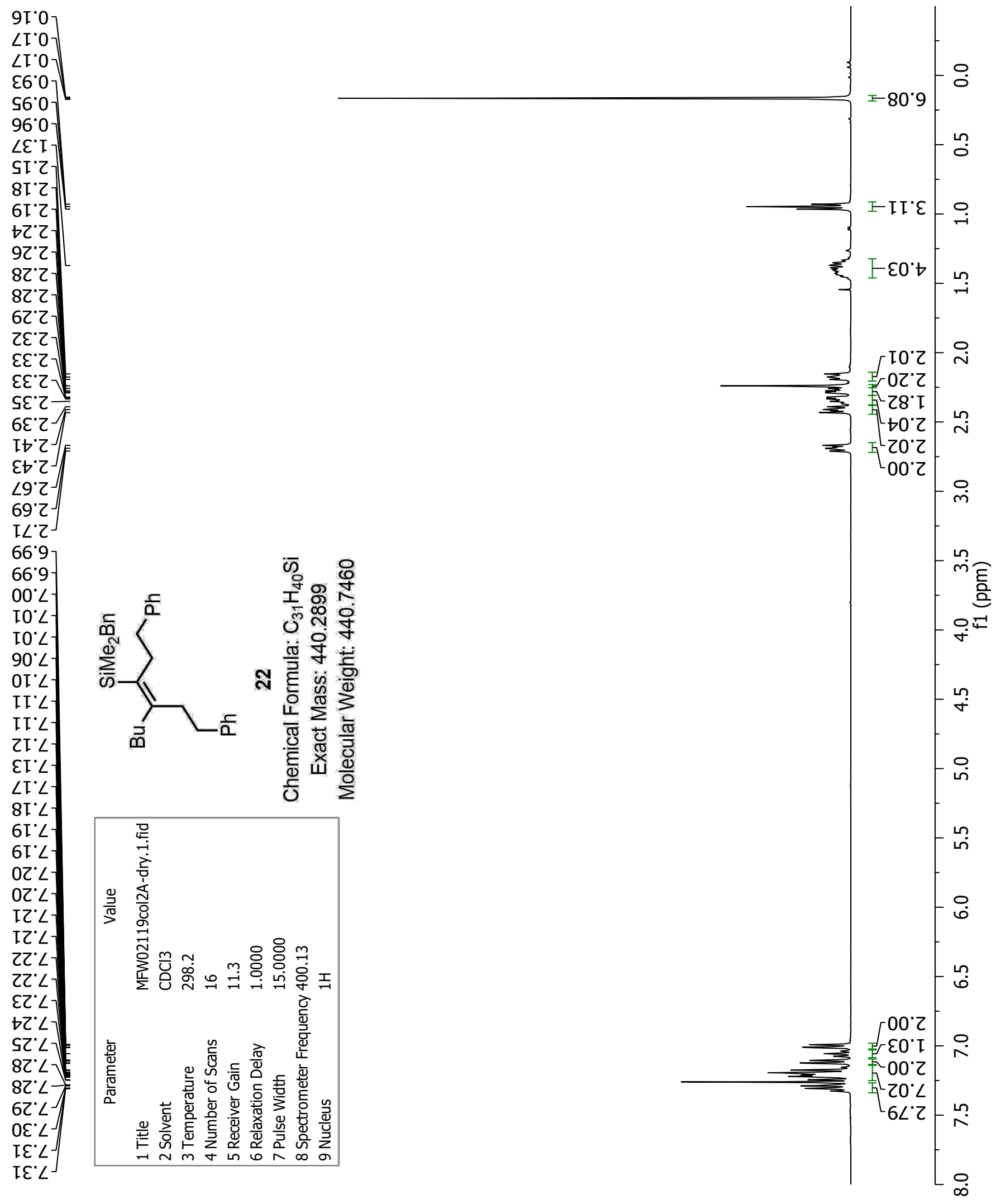



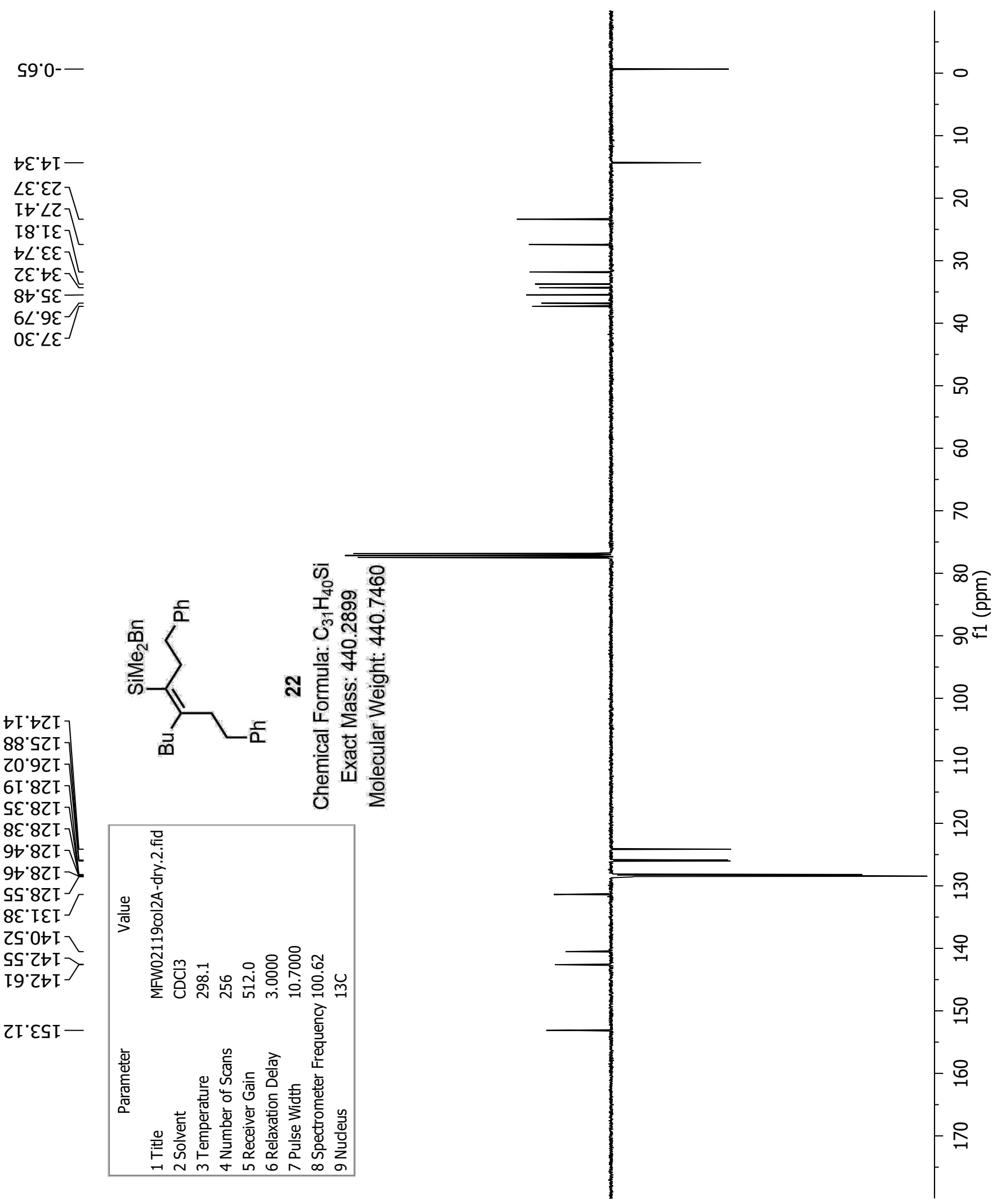

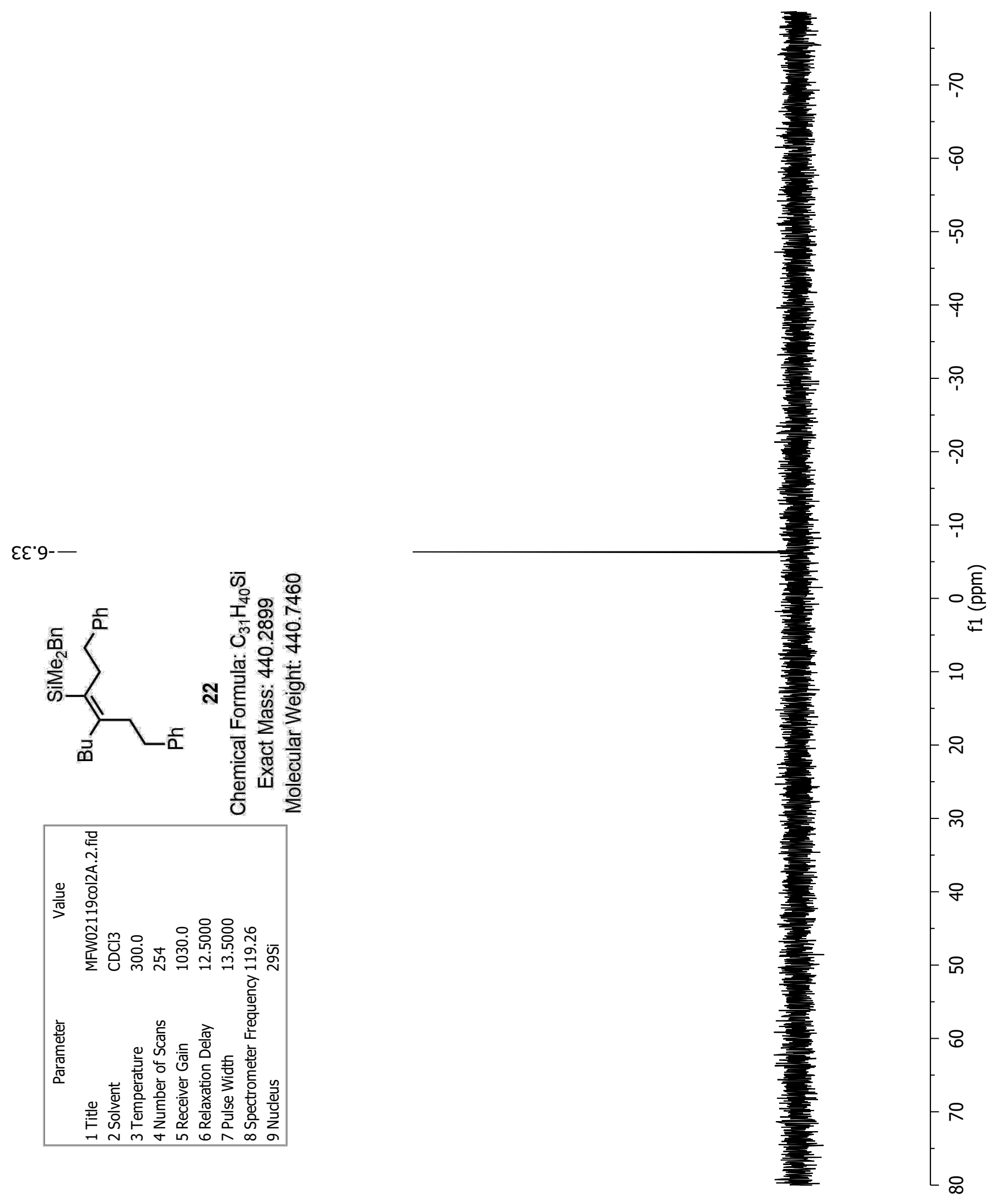


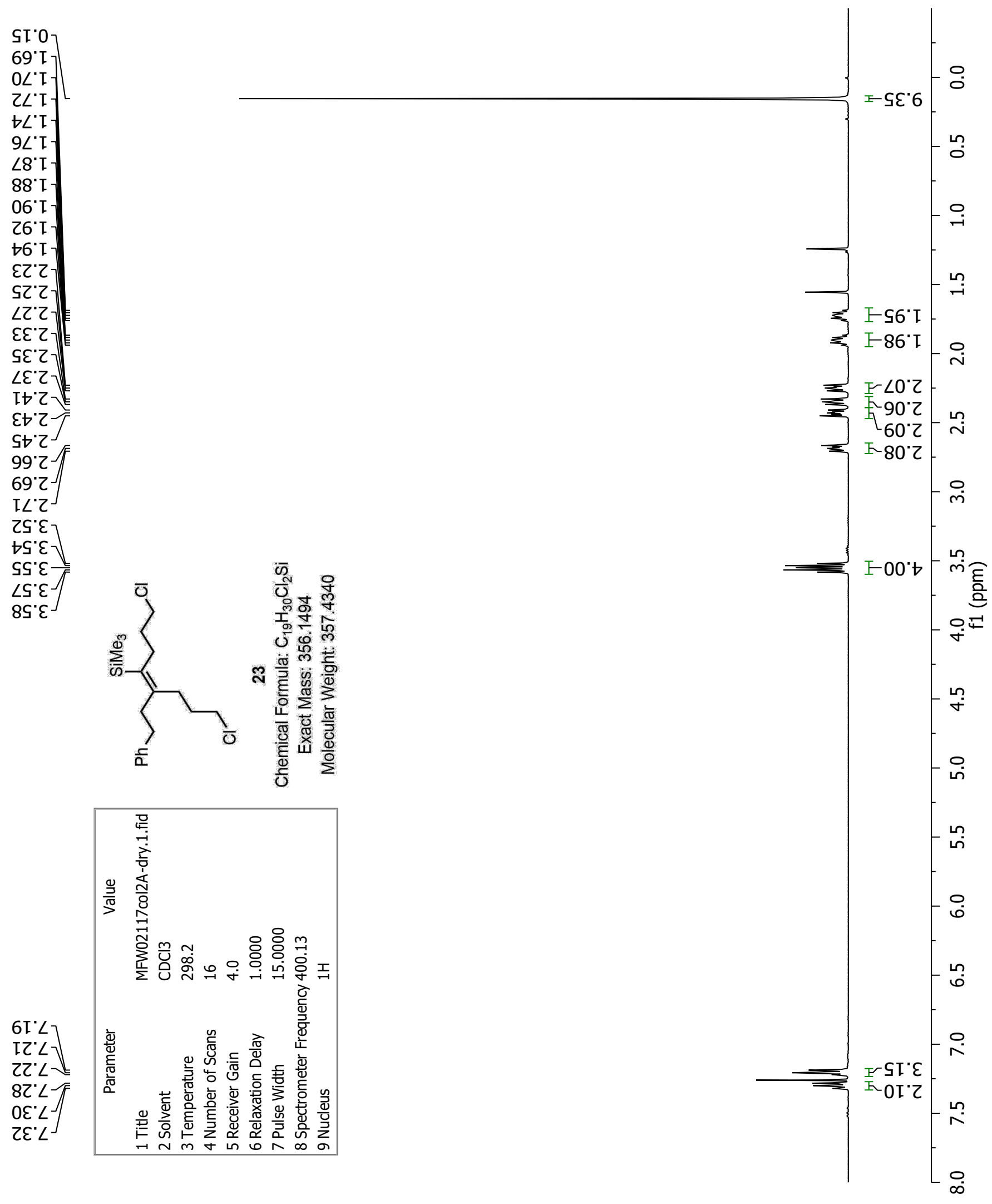



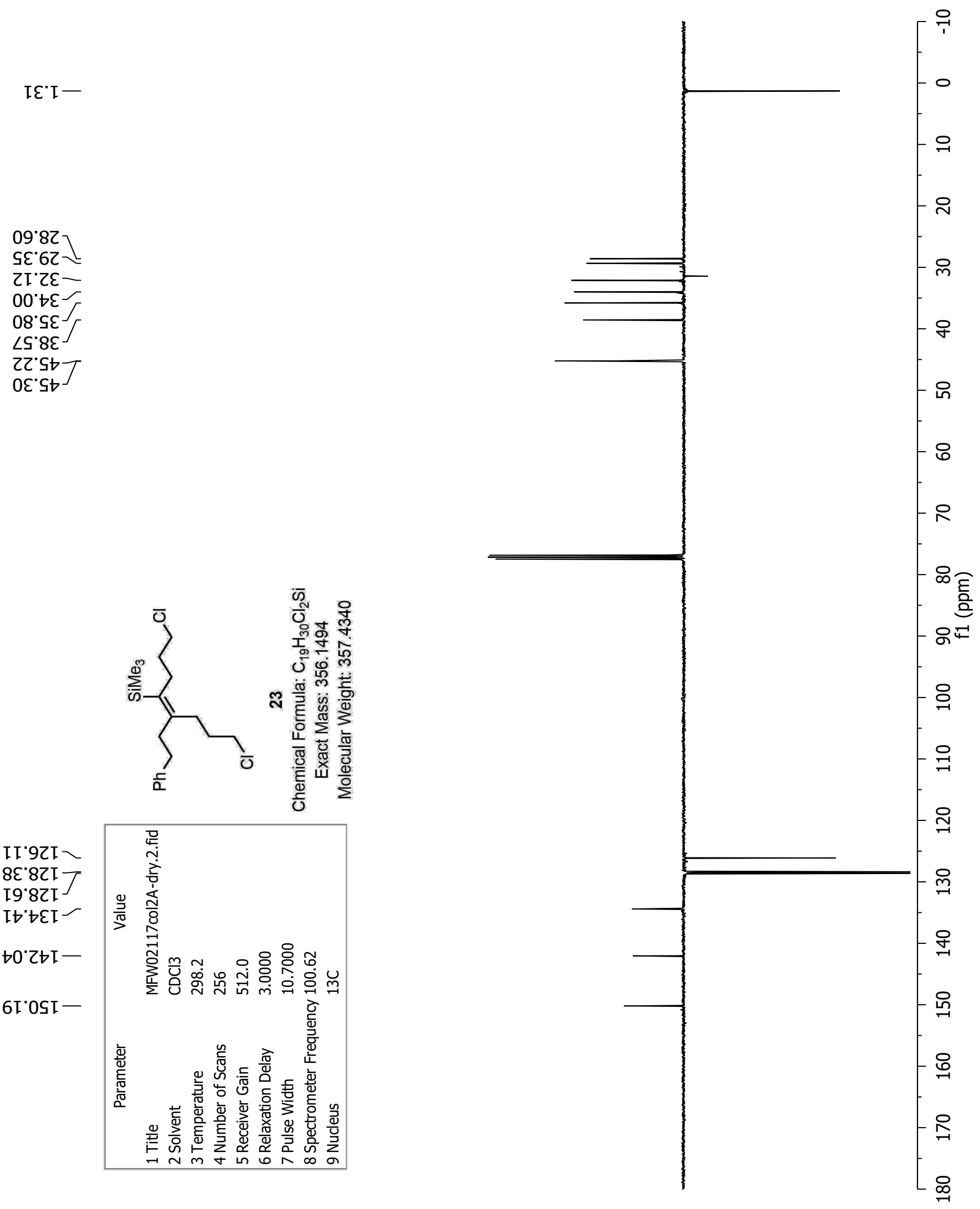

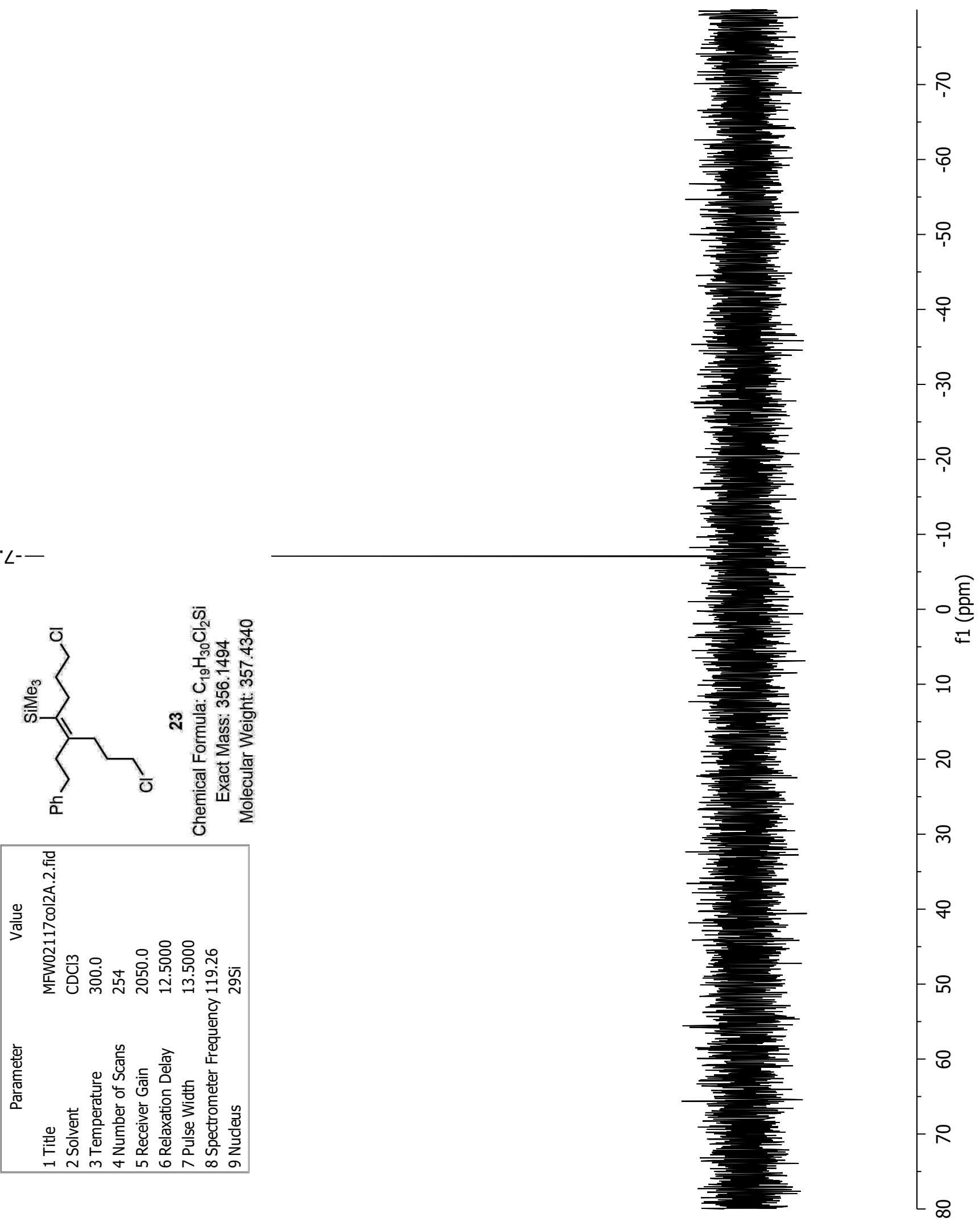


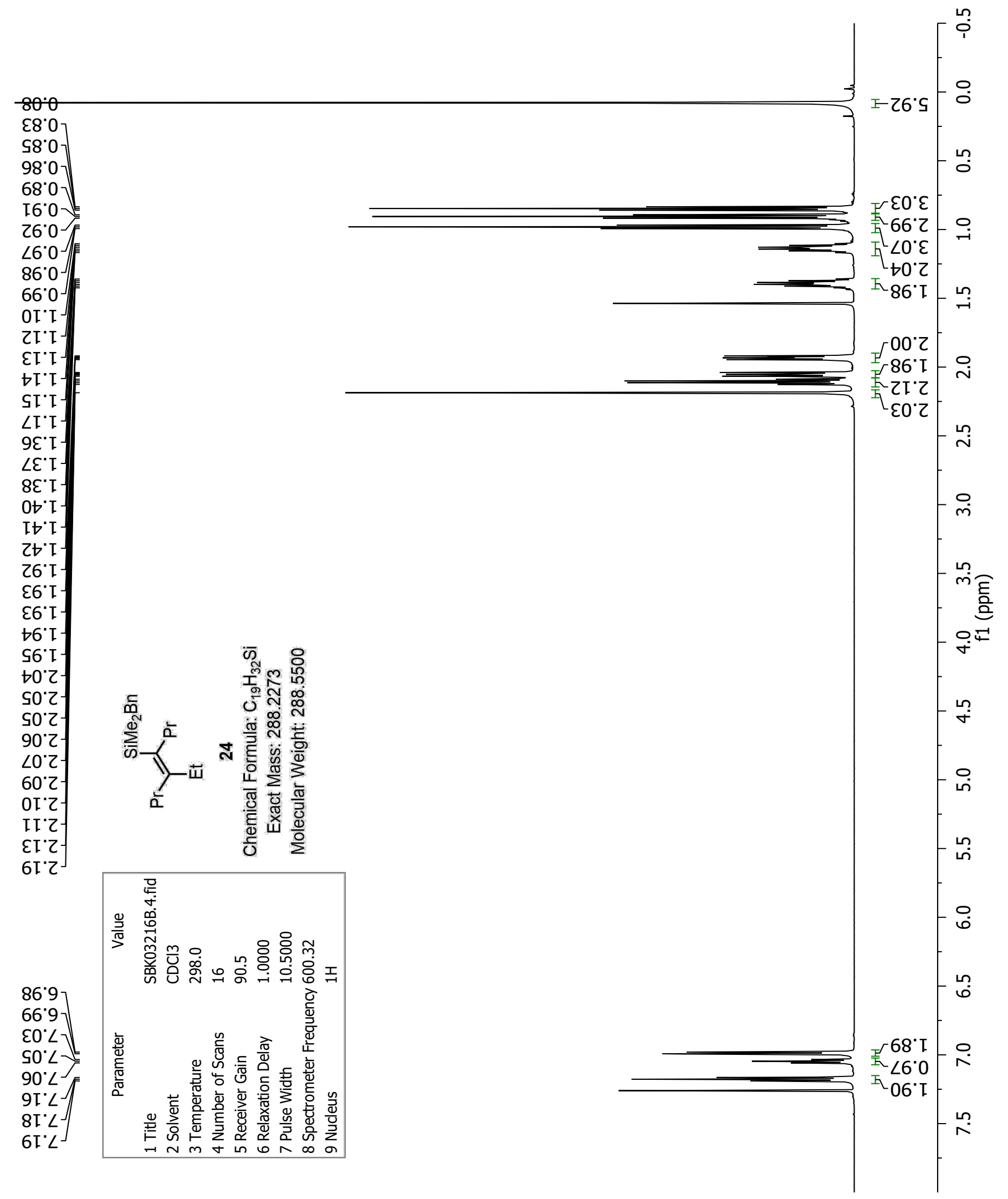



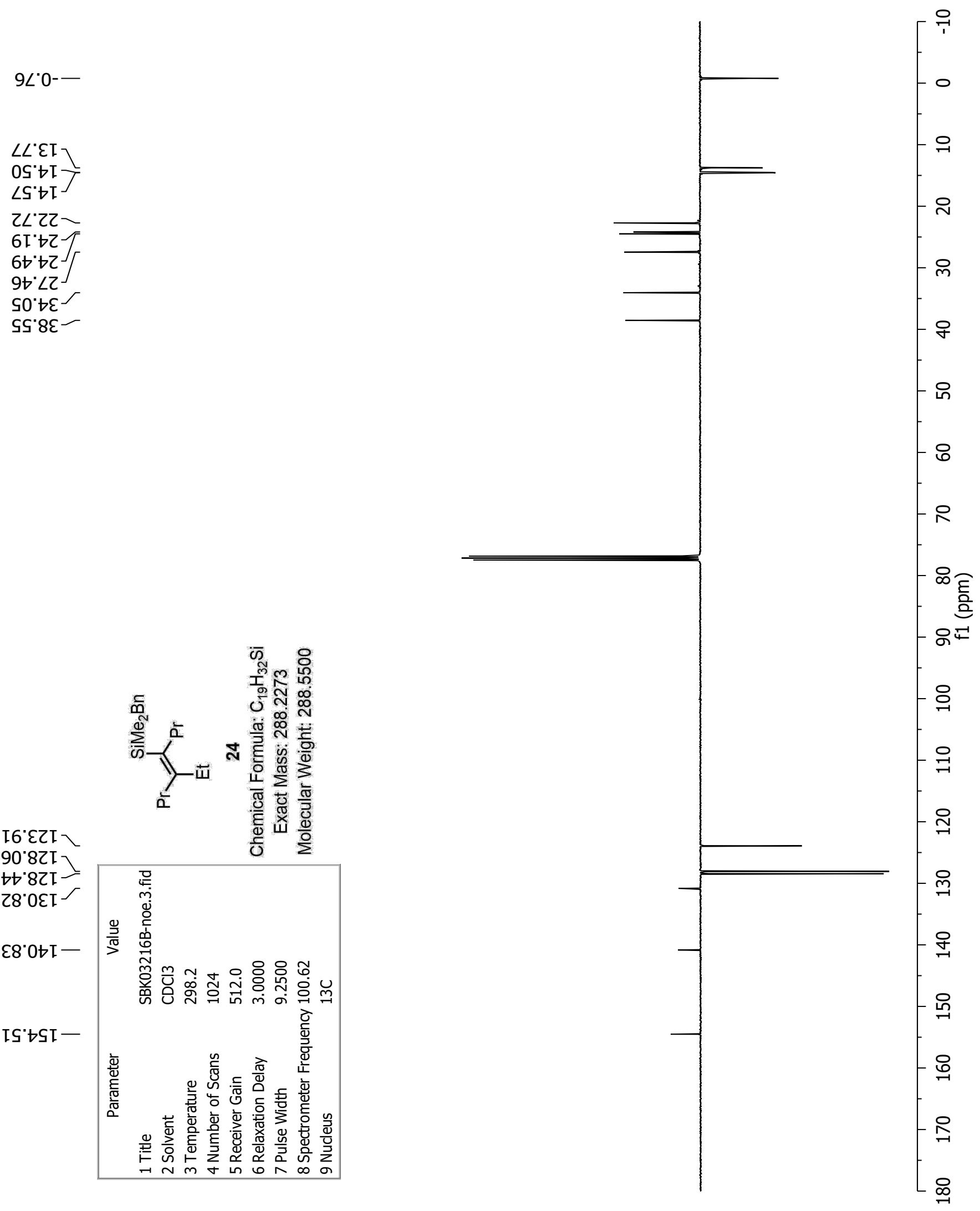

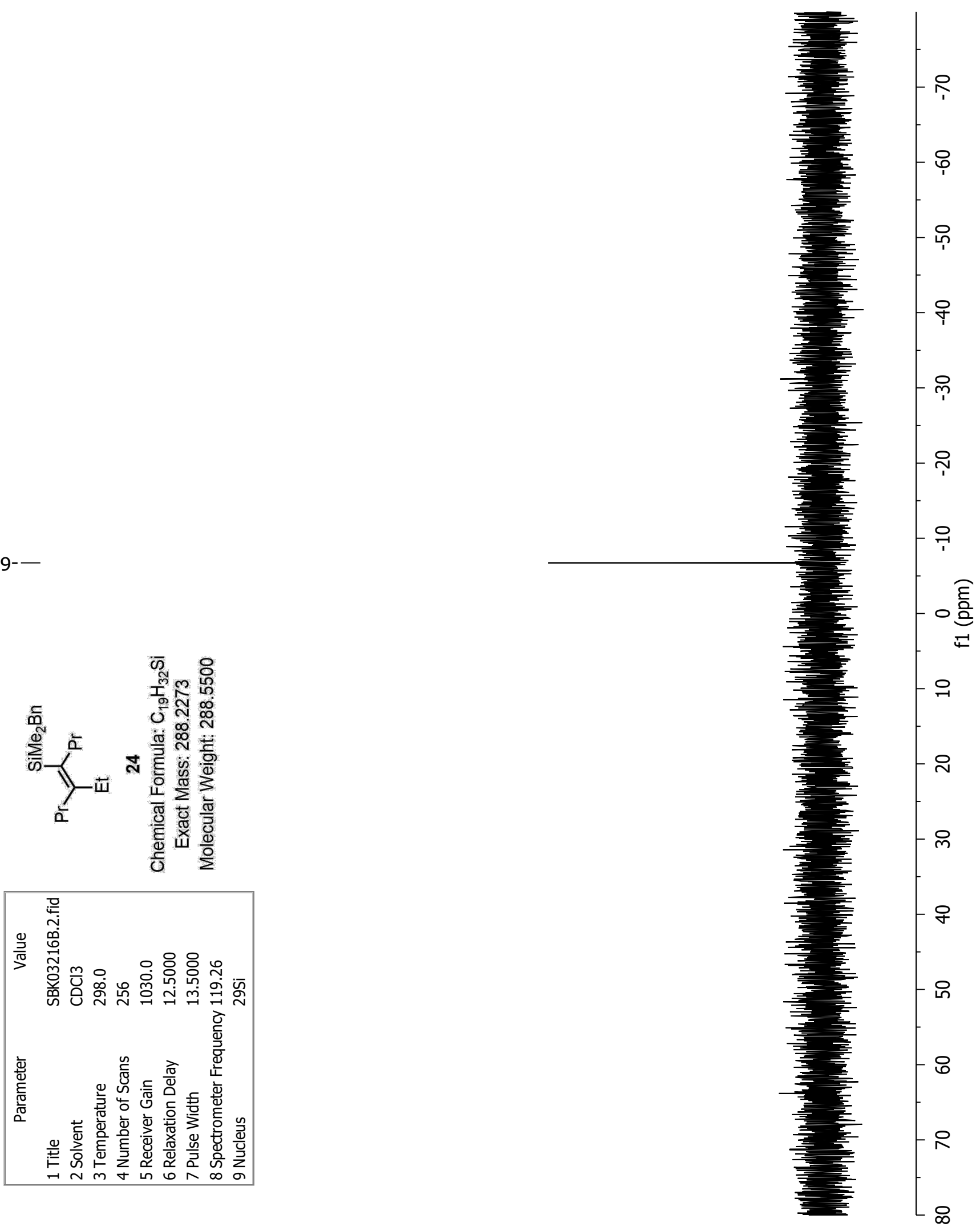


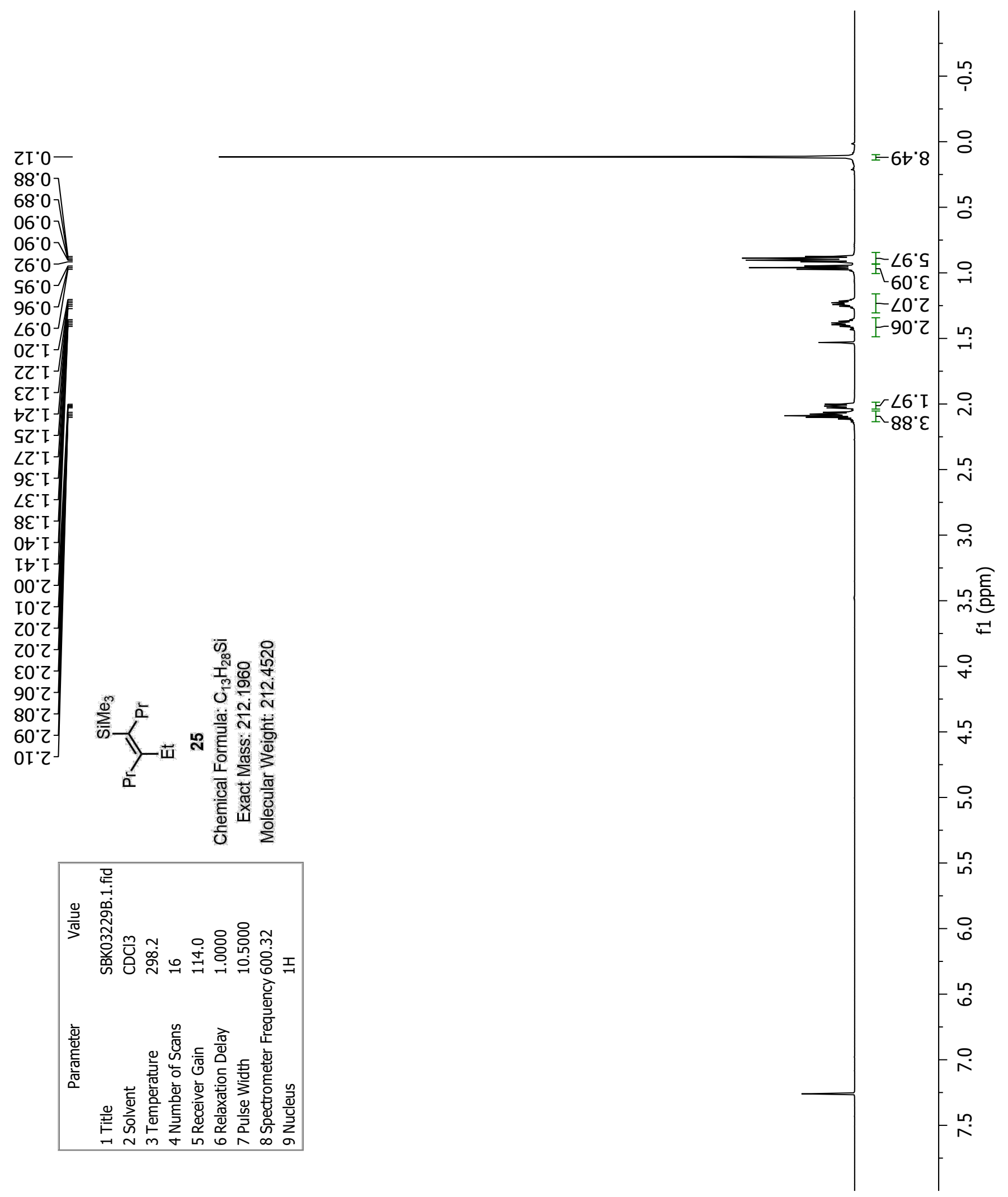



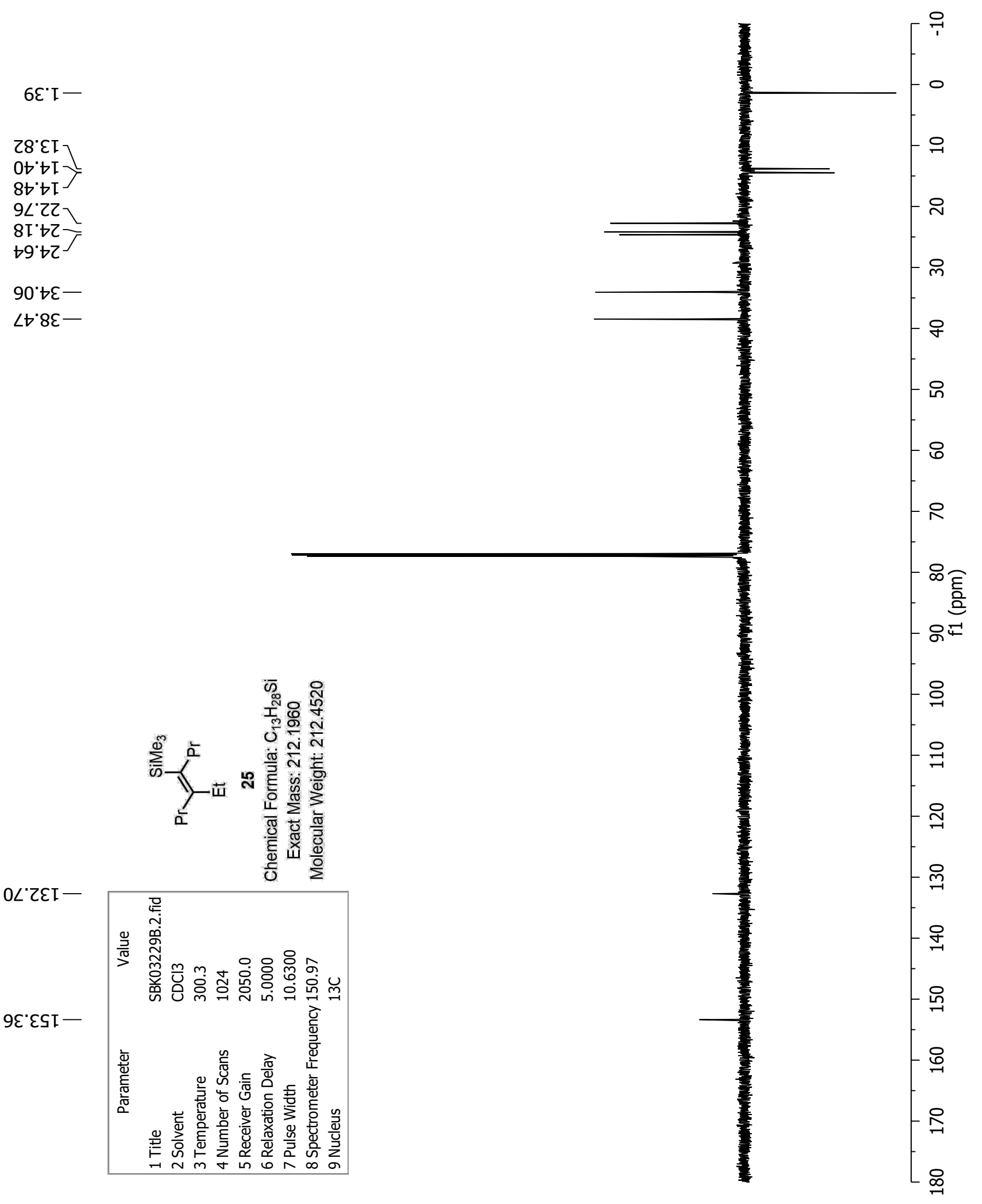


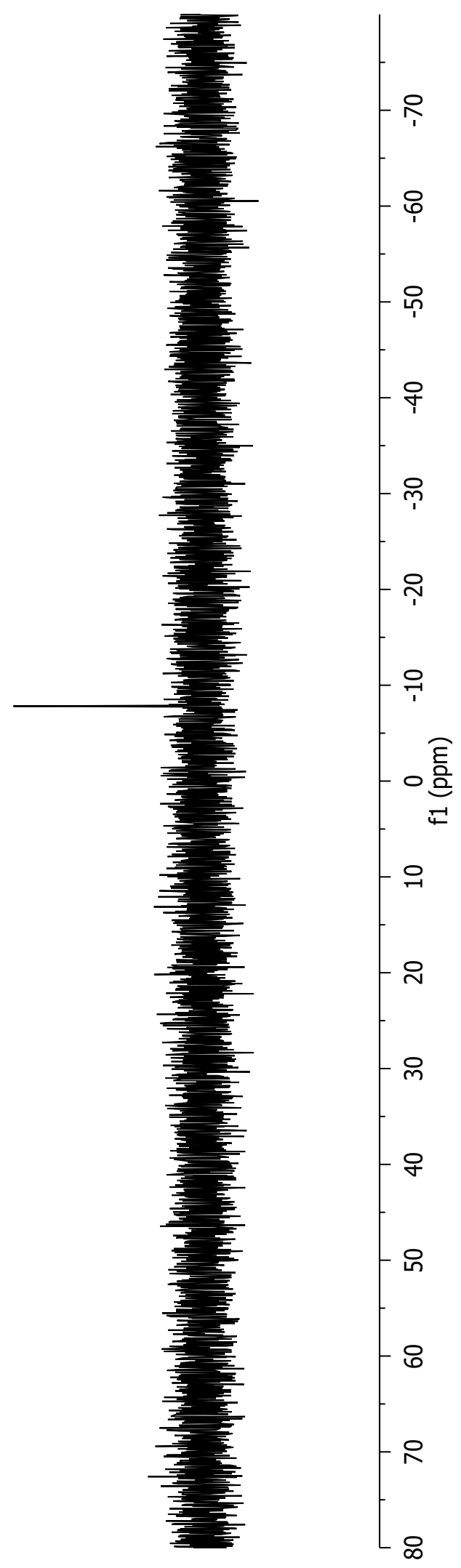



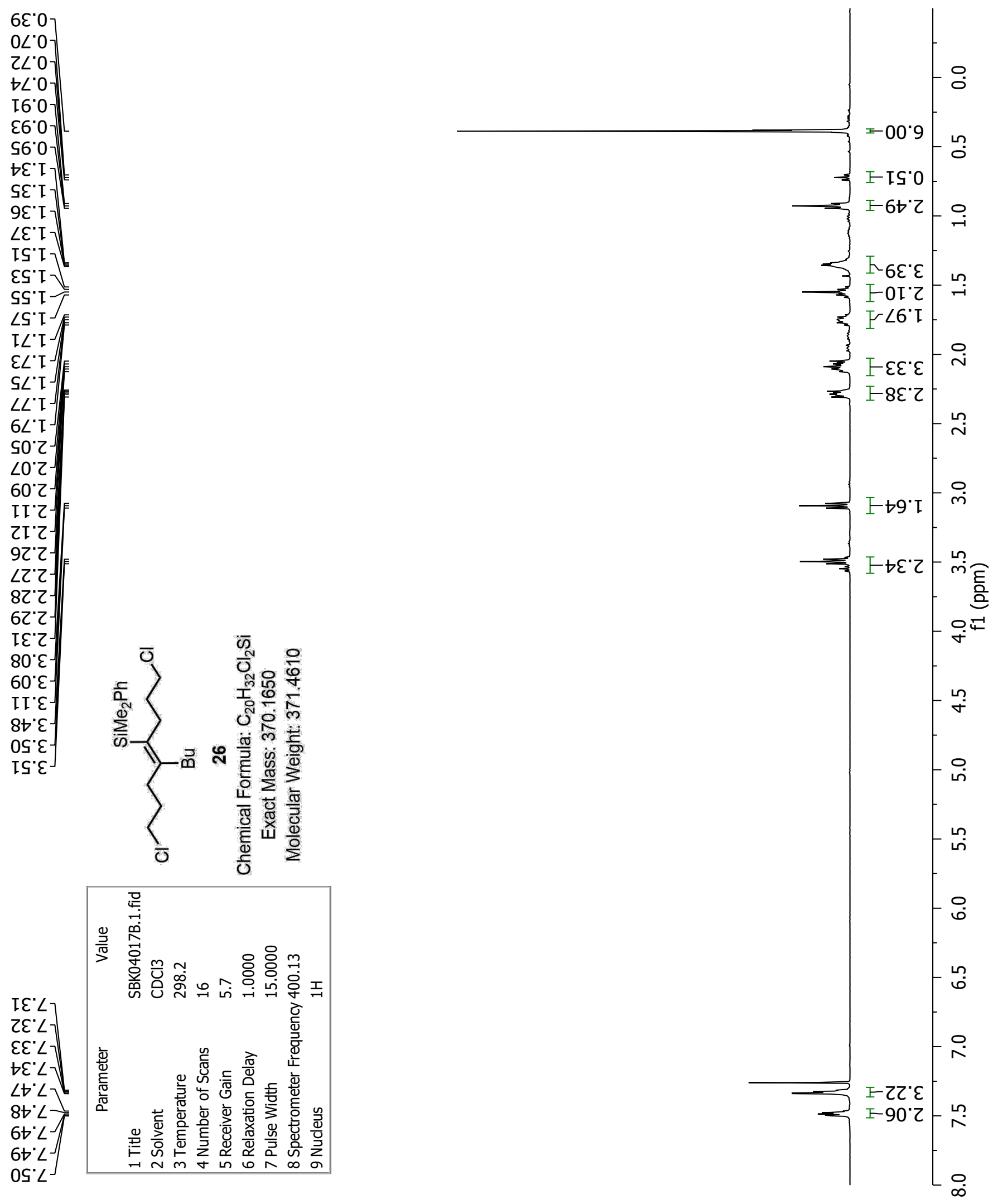

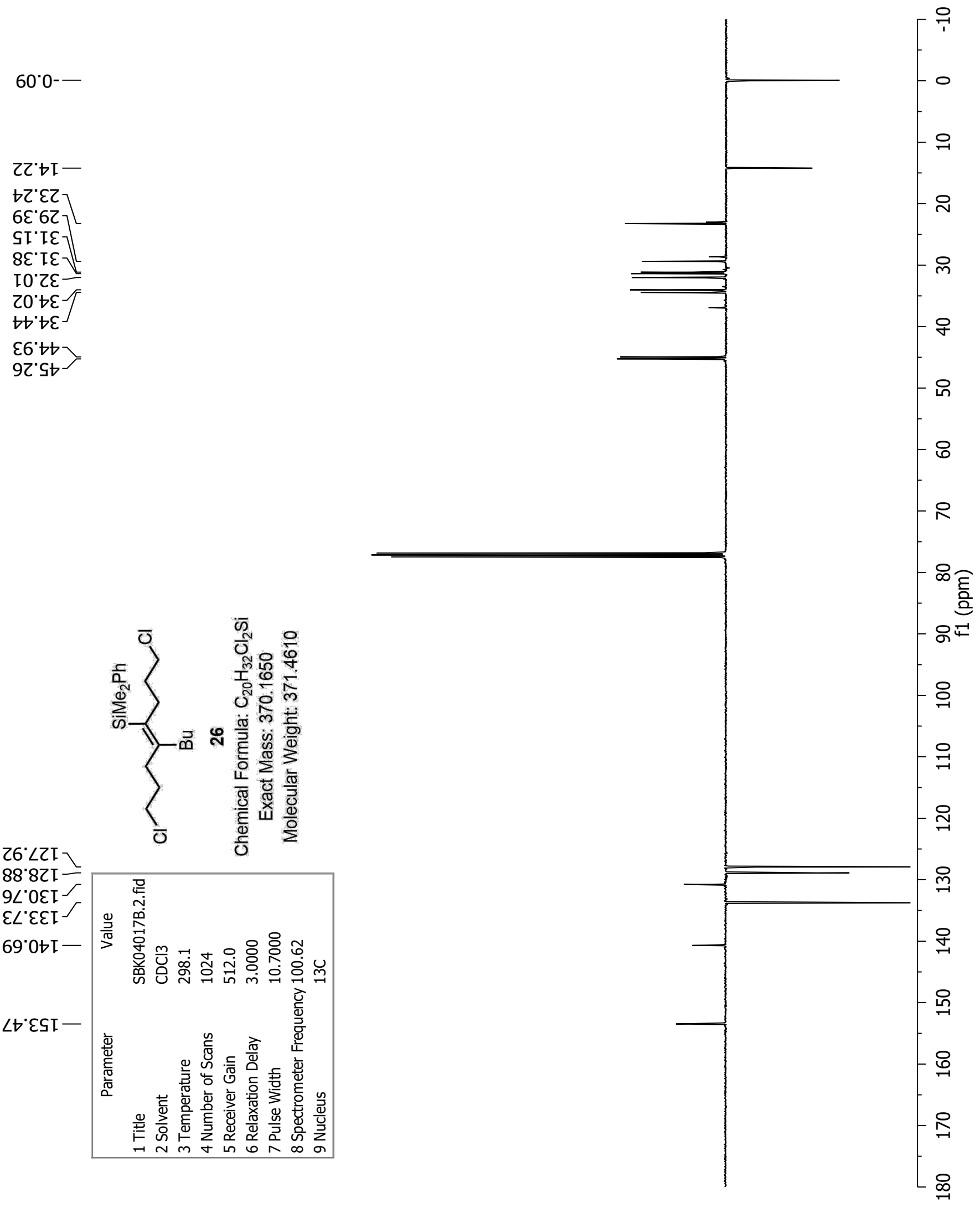

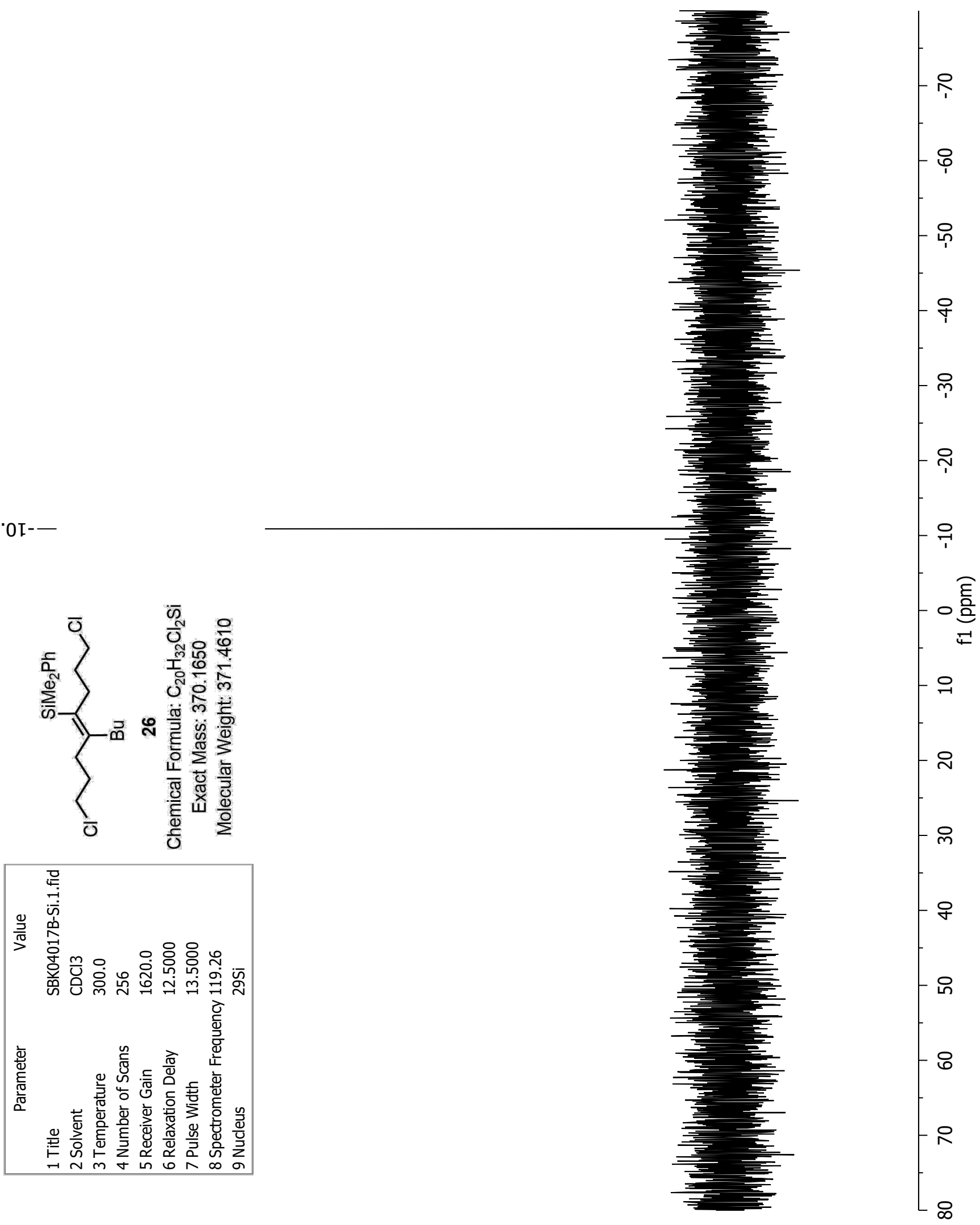

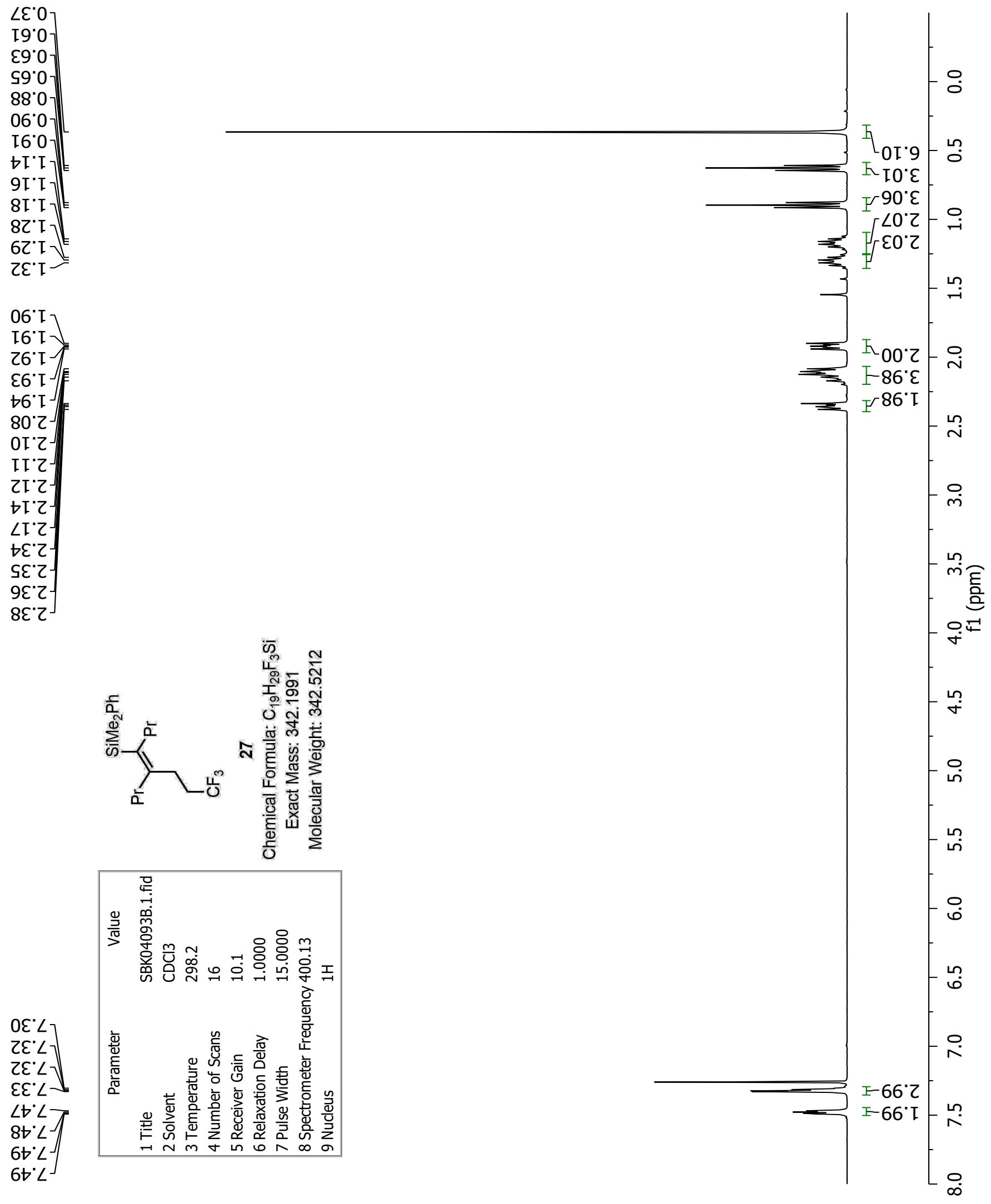


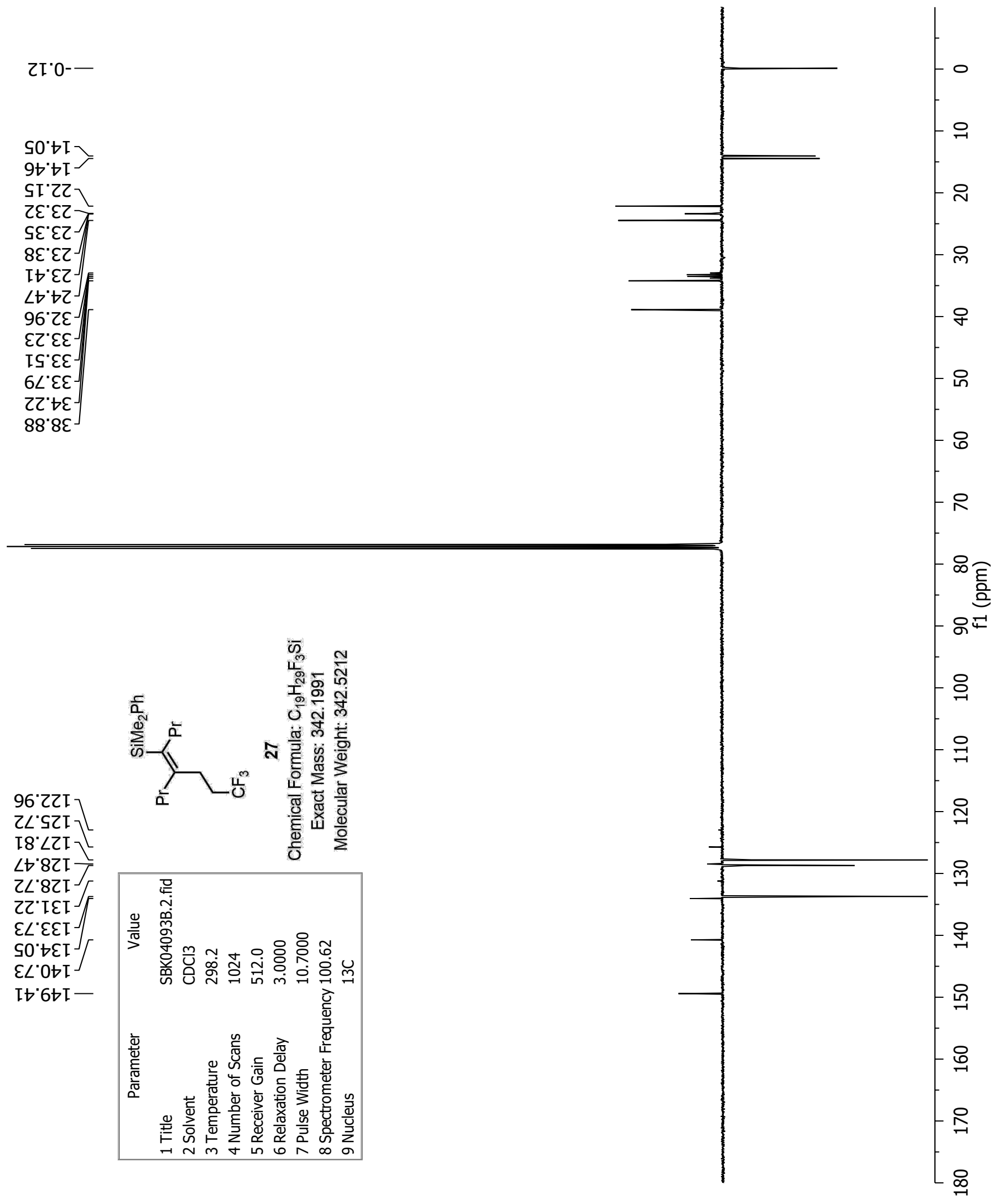




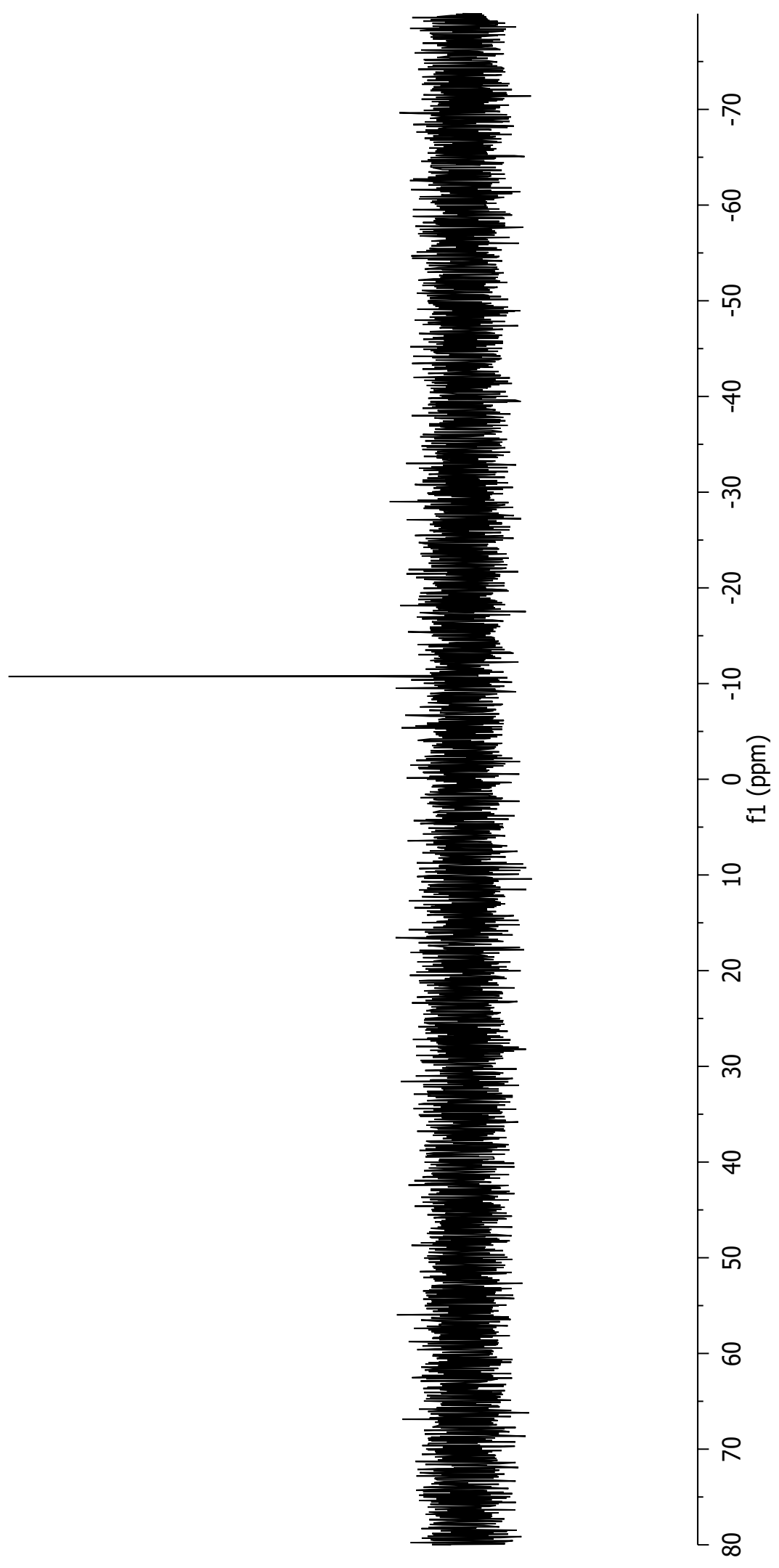




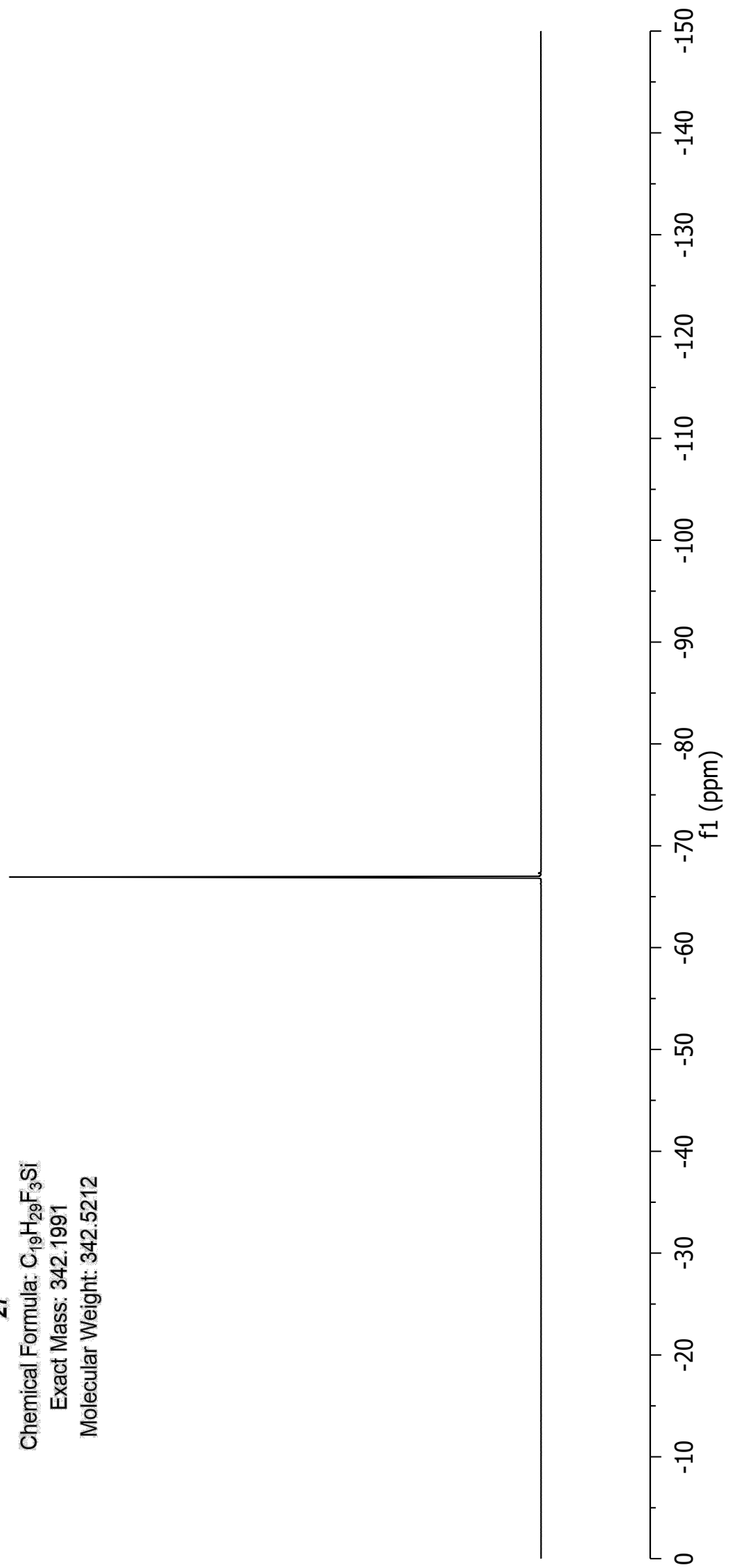



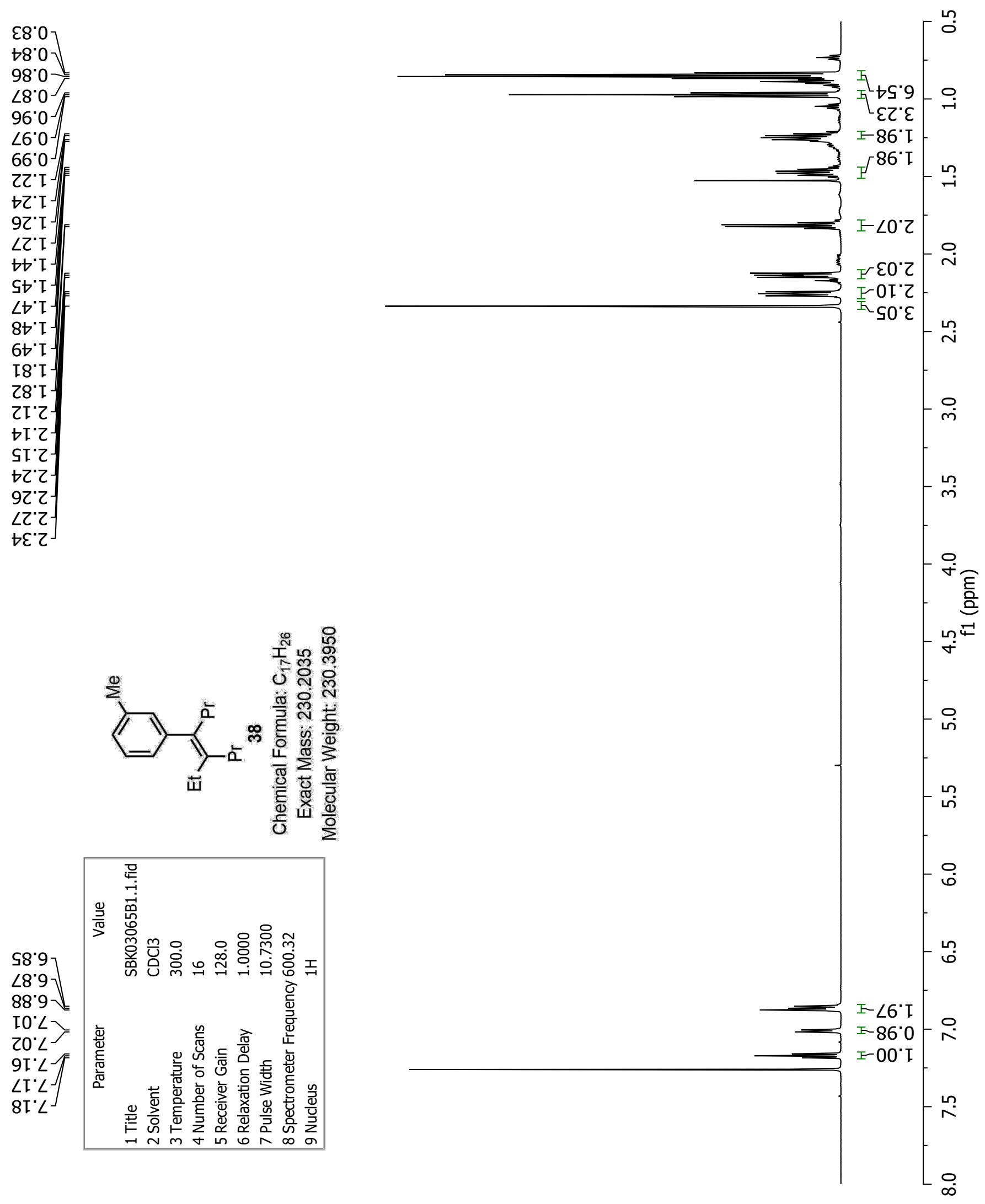

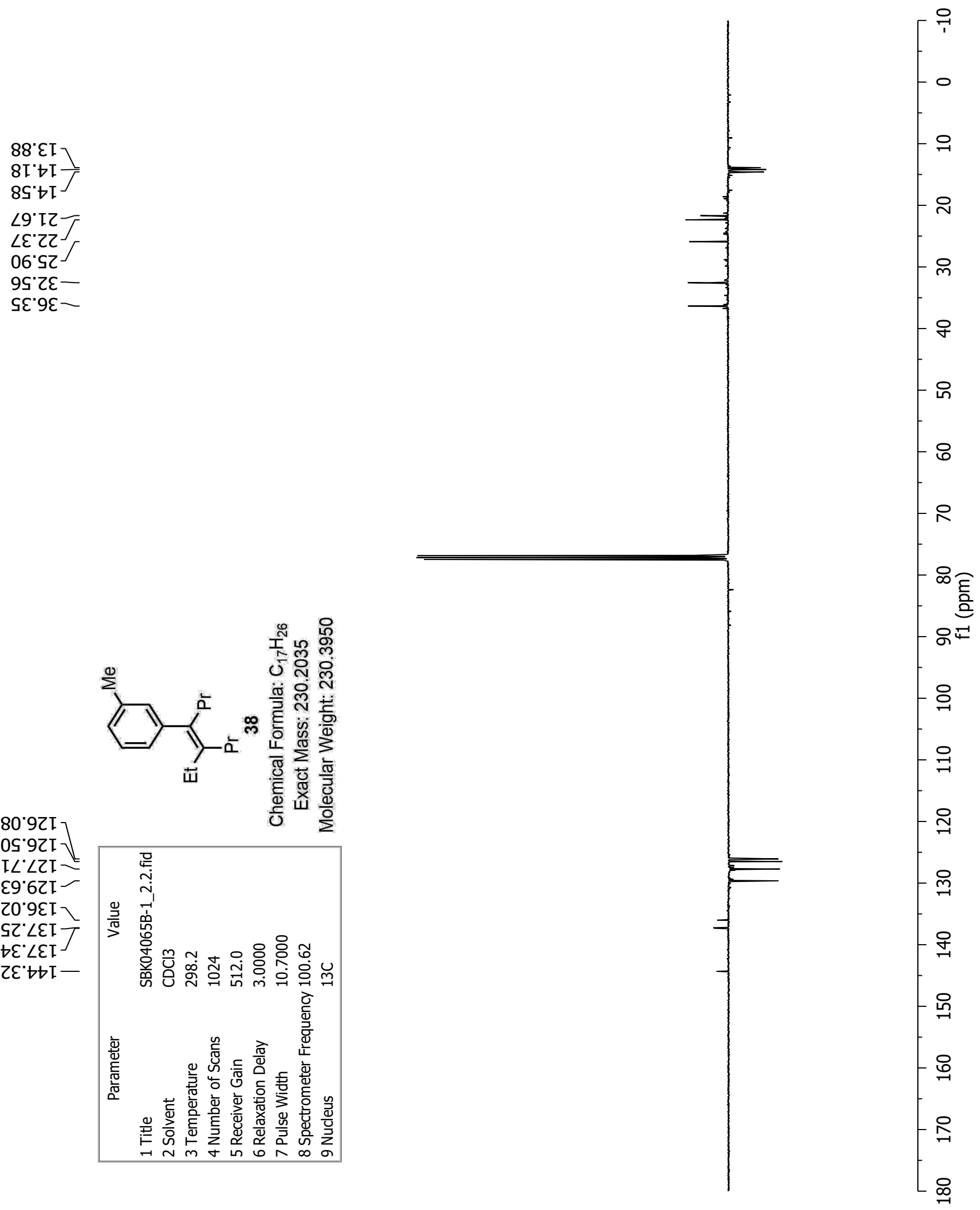